\title{
Study and Characterization of Hybrid Organic-Inorganic Perovskites for Solar Cells Applications
}

\author{
Author \\ Erika Vega Fleitas
}

Supervisors

Prof. Miguel A. Mollar García

Prof. Bernabé Marí Soucase

Valencia, July 2018

A dissertation submitted in fulfillment of the requirements for the Degree of Doctor of Philosophy 

To my mothen Manganita 



\section{Acknowledgments}

In the first place I would like to thank my thesis directors, Miguel Mollar and Bernabé Marí, for the patience and collaboration offered during my thesis. I would also like to thank them for their advice, discussions, scientific support and, above all, for giving me the opportunity to investigate a completely new topic for all of us.

I would also like to thank all the members of the Physics Department of the ETSID (UPV) for their support and understanding. Particularly, José Luis, technician of the department, for his predisposition and help during all these years. I am deeply grateful to all my colleagues and researchers from GOPS: Shafi Ullah, Suzan Saber, Krishna Raj, Bechir Slimi and Hanif Ullah for all the good times we have spent together during this entire scientific and personal journey. I would also like to thank here Jesús Cembrero, for all the help provided and his kindness and moreover, to Mari Carmen, the person in charge of cleaning, for her help in the laboratory, her impeccable work and for taking care of me. Also, I would like to thank the Institute of Design and Manufacturing for giving me the opportunity to belong to their GOPS team, especially Vicente Franch and Juan Antonio García for helping me solve problems whenever I needed it, and Mario Lledó for his kindness and the administrative information provided anytime I had doubts.

Special thanks to Dra. Estela Calixto for inviting me to do a research stay at the Institute of Applied Physics (BUAP-Mexico), where I enriched 
myself both scientifically and culturally, and to Dr. Antonio Mendez for his patience and time spent sharing his knowledge about photoluminescence, for his advice and talks on politics, history and life in general. I really appreciate their scientific expertise and profound knowledge, which helped me immensely during my thesis.

I am deeply grateful to Francisco Cataño, for sharing all his experience with $\mathrm{ZnO}$ with me and for the great moments and laughs together during his stay in Valencia. I would like to thank my colleague Lahoucine Atourki, for his enthusiasm, perseverance and talent. It was a pleasure to share our meal times, discussing about science, politics and life.

Thanks to the whole team of the Microscopy Service of the UPV for everything they taught me, for the patience and willingness to fulfill my requests, as well as for their tips and tricks to facilitate my learning.

I would also like to thank Prof. Juan Bisquert, director of the Advanced Materials Institute (INAM, University of Castellón) and his team, for inviting me to make a research stay. There, I had the opportunity to learn a lot in the development of devices with high performance and to meet Jesús Rodríguez who, in addition to an admirable scientific colleague, is a great friend. Nor will I forget the moments spent with Marta, Zahra, Ehsan, Ana, Azhar, Bruno, etc.

Thanks to my family, especially to my mother, brother, niece and uncle Carlos for their love and unconditional support throughout my student and research stage, and to my friends who help me just by being there and encourage me when I need it, and, finally my life partner, for his understanding, support and encouragement, and for being there in every moment lived during all these years.

Thank you all! 


\section{Abstract}

Organic-inorganic methylammonium lead halides perovskites $\left(M A P b X_{3}\right)$ and their mixtures have shown optimal optoelectronic properties as an ideal absorber for photovoltaic applications. In the last decade, solar devices based on perovskite have evolved rapidly, going from an initial efficiency of only $3.9 \%$ in 2009, to an efficiency of $22.7 \%$ in 2017 and being, at the same time, more cost-effective than silicon solar cells. However, one of the main disadvantages when using perovskite absorbents in photovoltaic devices is their low stability. In general, cells that show high performance lose their efficiency and degrade rapidly. For these materials to be scalable it is necessary to carry out in-depth studies aiming at improved efficiency and stability.

One of the main sources to improve stability and efficiency is compositional engineering, a strategy employed in the elaboration of this thesis, consisting of the investigation and improvement of the optoelectronic and morphological properties, derived from the substitution and / or combination of cations and anions, which constitute the perovskite material.

Pure powders of perovskite $M A P b X_{3}$ were synthesized, for $X=I, B r, C l$, from which pure and mixed $M A P b X_{3-x} Y_{x}$ films were prepared for $X, Y=$ $\mathrm{I}, \mathrm{Br}, \mathrm{Cl}$, in order to improve their optoelectronic and structural properties. By means of X-ray diffraction analysis, the structural properties of crystalline powders and pure and mixed films were studied. Employing UV-vis and photoluminescence analysis, it was observed that the absorption range varied 
along the visible spectrum as a function of the halide content in the thin films. Both, photoluminescence and differential scanning calorimetry analysis showed the changes of phase of the pure perovskites at different temperatures. FESEM characterization of the pure perovskites showed the morphological differences between the powders and the films.

Following this line of research, mixed perovskites of iodine-bromine with a bromine content of up to $33 \%$ were studied in more detail. The bandgap was tuned to avoid significant losses in absorption and improve the optoelectronic, structural and morphological properties.

Despite the excellent optoelectronic properties of the methylammonium perovskite, the presence of the organic cation decreases its stability, which prompted research into the use of other inorganic cations. Cesium perovskites, $\mathrm{CsPbX} X_{3}$, are a very promising alternative, and for this reason we synthesized thin films of mixed cesium perovskites, $C s P b B r_{3-x} I_{x}$, to determine the effects of the partial substitution of iodine on physical properties and stability. Films with a very good resistance to moisture and temperature were obtained, which will favor the application of this type of perovskites in the photovoltaic field.

The partial replacement of the methylammonium cation with other organic cations, such as guanidinium $(G A)$ and imidiazolium $(I M)$, was also studied, showing that small amounts of guanidinium significantly improve the stability of the films and their morphology. It was established that the solubility limit of guanidinium is approximately 20\%, and the crystalline structure of the mixtures was determined. An increase in the intensity of the photoluminescence peak for mixtures below the solubility limit was observed. Similar results were obtained for the substitution of methylammonium with small amounts of imidazolium. X-ray diffraction analyzes established the solubility limit at approximately $10 \%$ and an 
improvement in crystallinity. Photoluminescence results suggest that small amounts of imidazolium significantly reduce nonradiative recombinations, acting as an effective passivator.

Finally, the manufacturing process of devices based on $\mathrm{MAPbI}_{3}$ and synthesized according to environmental conditions, especially relative humidity and using diethyl ether as anti-solvent is shown. The devices presented a maximum efficiency of $14.73 \%$, proving that the oxidation of spiro-OMeTAD, under controlled humidity conditions, can improve efficiency. The devices studied showed similar efficiencies, which demonstrate good reproducibility. 



\section{Resumen}

Las perovskitas orgánicas-inorgánicas de haluros de metilamonio y plomo $\left(M A P b X_{3}\right)$ y sus mezclas han mostrado propiedades optoelectrónicas óptimas como absorbente ideal para aplicaciones fotovoltaicas. En la última década, los dispositivos solares basados en perovskita han evolucionado rápidamente, pasando de una eficiencia inicial de tan sólo el $3.9 \%$ en 2009, hasta alcanzar una eficiencia del $22.7 \%$ en 2017 y, además, teniendo un coste de fabricación más bajo que las células solares de silicio.

Sin embargo, una de las principales desventajas del uso de absorbentes de perovskita en dispositivos fotovoltaicos es su baja estabilidad. Por lo general, las células que muestran un alto rendimiento, pierden su eficiencia y se degradan rápidamente. Para que estos materiales puedan ser producidos industrialmente a gran escala es necesario realizar estudios en profundidad que mejoren la eficiencia y estabilidad.

Una de las principales vías de mejora en la estabilidad y en la eficiencia es la ingeniería composicional, estrategia que hemos empleado en la elaboración de esta tesis y que consiste en la investigación y mejora de las propiedades optoelectrónicas y morfológicas, derivadas de la sustitución y/o combinación de cationes y aniones, que constituyen el material de perovskita.

Se han sintetizado polvos puros de perovskita $M A P b X_{3}$, para $X=$ $\mathrm{I}, \mathrm{Br}, \mathrm{Cl}$, a partir de los cuales se prepararon capas puras y mixtas $\mathrm{MAPbX}_{3-x} Y_{x}$ para $X, Y=I, B r, C l$, con el objetivo de mejorar sus 
propiedades optoelectrónicas y estructurales. Mediante análisis de difracción de rayos $\mathrm{X}$, se estudió las propiedades estructurales de los polvos cristalinos y capas puras y mixtas. Los análisis de UV-vis y fotoluminiscencia mostraron que el rango de absorción varía a lo largo del espectro visible en función del contenido del haluro en las capas. Los análisis de fotoluminiscencia y calorimetría diferencial de barrido muestran los cambios de fase de las perovskitas puras a distintas temperaturas, coincidiendo dichos cambios de fase en ambos análisis. Las micrografías FESEM de las perovskitas puras muestran las diferencias morfológicas entre los polvos y capas.

Siguiendo esta línea de investigación, se estudiaron con más detalle las perovskitas mixtas de yodo-bromo, con un contenido de bromo de hasta el $33 \%$, consiguiéndose ajustar el bandgap para evitar pérdidas significativas en la absorción y mejorar las propiedades optoelectrónicas, estructurales y morfológicas.

A pesar de las excelentes propiedades optoelectrónicas de las perovskitas de metilamonio, la presencia del catión orgánico disminuye su estabilidad, lo que ha llevado a investigar el uso de otros cationes inorgánicos. Las perovskitas de cesio, $\mathrm{CsPbX}_{3}$, son una alternativa muy prometedora, y por esta razón hemos sintetizado capas finas de perovskitas de cesio mixtas, $\mathrm{CsPbBr}_{3-x} I_{x}$, para determinar los efectos que produce la sustitución parcial del yodo en las propiedades físicas y la estabilidad. Se obtuvieron capas con una buena resistencia a la humedad y a la temperatura, que favorecerá la aplicación de este tipo de perovskitas en el campo fotovoltaico.

Se ha estudiado, además, la sustitución parcial del catión de metilamonio con otros cationes orgánicos, como el guanidinio (GA) e imidiazolio (IM). Se ha demostrado que pequeñas cantidades de guanidinio mejoran significativamente la estabilidad de las capas y su morfología. Se ha establecido que el límite de 
solubilidad del guanidinio es del 20\%, aproximadamente, y se ha determinado la estructura cristalina de las mezclas. Se ha observado un aumento en la intensidad del pico de fotoluminiscencia para mezclas por debajo del límite de solubilidad.

Se obtuvieron resultados similares para la sustitución del metilamonio con pequeñas cantidades de imidazolio. Los análisis de difracción de rayos $\mathrm{X}$ establecieron el límite de solubilidad en aproximadamente el 10\% y una mejora en la cristalinidad. Los resultados de fotoluminiscencia sugieren que pequeñas cantidades de imidazolio reducen significativamente las recombinaciones no radiativas, actuando como un pasivador efectivo.

Finalmente, se muestra el proceso de fabricación de dispositivos basados en $\mathrm{MAPbI}_{3}$ y sintetizados en función de las condiciones ambientales, especialmente la humedad relativa y empleando el dietil éter como anti-solvente. Los dispositivos mostraron una eficiencia máxima del $14.73 \%$. Se ha probado que la oxidación del spiro-OMeTAD, bajo condiciones controladas de humedad puede mejorar la eficiencia. Los dispositivos estudiados mostraron eficiencias similares, lo que demuestra la buena reproducibilidad. 



\section{Resum}

Les perovskites orgàniques-inorgàniques de halurs de metilamoni i plom $\left(M A P b X_{3}\right)$ i les seues mescles han mostrat propietats optoelectròniques òptimes com a absorbent ideal per a aplicacions fotovoltaiques. En l'última dècada, els dispositius solars basats en perovskita han evolucionat ràpidament, passant d'una eficiència inicial de tan sols el $3.9 \%$ en 2009, fins a aconseguir una eficiència del $22.7 \%$ en 2017 , i, a més a més, tenint un cost de fabricació més baix que les cèl.lules solars de silici.

No obstant això, un dels principals desavantatges de l'ús de absorbents de perovskita en dispositius fotovoltaics és la seua baixa estabilitat. En general, les cèl.lules que mostren un alt rendiment, perden la seua eficiència i es degraden ràpidament. Amb l'objectiu de que aquestos materials puguen ser produits industrialment a gran escala és necessari realitzar estudis en profunditat per tal de millorar la seua eficiència i estabilitat.

Una de les principals vies de millora en l'estabilitat i en l'eficiència és l'enginyeria composicional, estratègia que hem emprat en l'elaboració d'aquesta tesi i que consisteix en la investigació i la millora de les propietats optoelectròniques i morfològiques, derivades de la substitució i/o combinació de cations i anions, que constitueixen el material de perovskita.

S'han sintetitzat pols purs de perovskita $M A P b X_{3}$, per a $X=I, B r, C l$, a partir d'els quals es van preparar capes pures i mixtes $M A P b X_{3-x} Y_{x}$ per a $X, Y=I, B r, C l$, amb l'objectiu de millorar les seues propietats 
optoelectròniques i estructurals. Mitjançant anàlisi de difracció de raigs X, es van estudiar les propietats estructurals del pols cristalins i capes pures i mixtes. Mitjançant les anàlisis d'UV-vis i fotoluminiscència, es va observar que el rang d'absorció varia al llarg de l'espectre visible en funció del contingut de l'halur en les capes. Les anàlisis de fotoluminiscència i calorimetria diferencial d'exploració mostren els canvis de fase de les perovskites pures a diferents temperatures, coincidint aquestos canvis de fase en totes dues anàlisis. La caracterització mitjançant FESEM de les perovskites pures, mostra les diferències morfològiques entre els pols i capes.

Seguint aquesta línia d'investigació, es van estudiar amb més detall les perovskites mixtes de iode-brom, amb un contingut de brom de fins el $33 \%$, aconseguint-se ajustar el bandgap per tal de evitar pèrdues significatives en l'absorció i millorar les propietats optoelectròniques, estructurals i morfològiques.

Malgrat les excel.lents propietats optoelectròniques de les perovskites de metilamoni, la presència del catió orgànic disminueix la seua estabilitat, la qual cosa ha portat a investigar l'ús d'altres cations inorgànics. Les perovskites de cesi, $\mathrm{CsPbX}$, són una alternativa molt prometedora, i per aquesta raó hem sintetitzat capes fines de perovskites de cesi mixtes, $C s \mathrm{PbBr}_{3-x} I_{x}$, per tal de determinar els efectes que produeix la substitució parcial del iode en les propietats físiques i l'estabilitat. Es van obtenir capes amb una molt bona resistència a la humitat i a la temperatura, la qual cosa afavorirà l'aplicació d'aquest tipus de perovskites en el camp fotovoltaic.

S'ha estudiat a més a més la substitució parcial del catió de metilamoni amb altres cations orgànics, com el guanidini (GA) i imidiazoli (IM). S'ha demostrat que petites quantitats de guanidini milloren significativament l'estabilitat de les capes i la seva morfologia. S'ha establert que el límit 
de solubilitat del guanidini es del $20 \%$, aproximadament, i s'ha determinat l'estructura cristal.lina de les mescles. S'ha observat un augment en la intensitat del pic de fotoluminiscència per a mescles per sota del límit de solubilitat.

Es van obtenir resultats similars per a la substitució del metilamoni amb petites quantitats de imidazoli. Les anàlisis de difracció de raigs $\mathrm{X}$ van establir el límit de solubilitat en aproximadament el 10\% i una millora en la cristalinitat. Els resultats de fotoluminiscència suggereixen que petites quantitats de imidazoli redueixen significativament les recombinacions no radiatives, actuant com un pasivador efectiu.

Finalment, es mostra el procés de fabricació de dispositius basats en $M A P b I_{3}$ i sintetitzats en funció de les condicions ambientals, especialment la humitat relativa i utilitzant el dietil èter com anti-solvent. Els dispositius van mostrar una eficiència màxima del $14.73 \%$. S'ha provat que l'oxidació del spiro-OMeTAD, sota condicions controlades d'humitat pot millorar l'eficiència. Els dispositius estudiats van mostrar eficiències similars, la qual cosa demostra la bona reproducibilitat. 



\section{Contents}

1 Introduction $\quad 1$

1.1 Global Energy Crisis . . . . . . . . . . . . . . . . 1

1.2 The Case for Solar Power . . . . . . . . . . . . . . . 5

1.3 Solar Cells ..................... 6

2 Organic-Inorganic Halide Perovskites 13

2.1 Organic-Inorganic Hybrid Materials . . . . . . . . . . . . . 13

2.2 Perovskites for Solar Cells . . . . . . . . . . . . . . . . . 14

2.2.1 Perovskite Material . . . . . . . . . . . . . 14

2.2.2 Perovskite-based Solar Cells . . . . . . . . . . 18

2.2.3 Crystal Structure . . . . . . . . . . . . 21

2.2.4 Stability and Compositional Engineering . . . . . . . . 22

2.3 Components of Typical Perovskite Solar Cells . . . . . . . . . . 38

2.3.1 The Compact Layer . . . . . . . . . . . . . . 38

2.3.2 The Mesoporous Layer . . . . . . . . . . . . . . . . 39

2.3.3 The Perovskite Absorber . . . . . . . . . . . 40

2.3.4 The Hole Transport Layer . . . . . . . . . . . . . . . . . 41

2.3.5 Back-contact ................. . . 42

2.4 Chemical and Physical Techniques . . . . . . . . . . . . 43

2.4.1 Solution Processes . . . . . . . . . . . . . 43 
2.4.2 Deposition Techniques .............. . 45

2.4.3 Morphology Control . . . . . . . . . . . . . 48

2.5 The Physics of Solar Cells . . . . . . . . . . . . . . . 52

2.5.1 Introduction ................ 52

2.5.2 Air Mass ................... . . . 54

2.5.3 Properties of Photovoltaics . . . . . . . . . . . 55

2.5.4 Perovskite Bandgap Origin . . . . . . . . . . . . 71

2.5.5 Hysteresis in Current-Voltage Measurements . . . . . . 73

3 Characterization Techniques $\quad 79$

$3.1 \quad X$-ray Diffraction . . . . . . . . . . . . . . . 79

3.2 Photoluminescence .................. . . 81

3.3 Atomic Force Microscope . . . . . . . . . . . . . . . . . . . . . 81

3.4 Field Emission Scanning Electron Microscope . . . . . . . . . . 82

3.5 Optical Spectroscopy . . . . . . . . . . . . . . . 82

4 Synthesis and Bandgap Tuning of $M_{A P b X_{3-x}} Y_{x} \quad(X, Y=$ $I, B r, C l)$ Hybrid Perovskites $\quad 85$

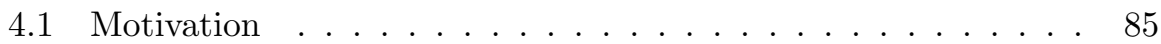

4.2 Experimental ........................ 88

4.2.1 Synthesis of Pure Perovskite Powders . . . . . . . . 88

4.2.2 Synthesis of Thin Film Perovskites . . . . . . . . . . . 88

4.3 Results and Discussion . . . . . . . . . . . . . . . . 89

4.3.1 X-ray Diffraction ............... 89

4.3.2 Absorbance ................... 97

4.3.3 Photoluminescence . . . . . . . . . . . . . . . . . 99

4.3.4 FESEM Analysis ............... 105

4.4 Conclusion ........................ 107 
5 Role of the Chemical Substitution in the Structural and Luminescence Properties of the Mixed Halide Perovskite $M A P b I_{3-x} B r_{x}(0 \leq x \leq 1)$ thin films

5.1 Motivation .......................... 109

5.2 Experimental Procedure . . . . . . . . . . . . . . 110

5.3 Results and Discussion . . . . . . . . . . . . . 111

5.3.1 X-ray Analysis ................. 111

5.3.2 FESEM and AFM Analyses . . . . . . . . . . . 114

5.3.3 $U V$-vis Spectra and Luminescence Properties . . . . . . 117

5.4 Conclusion ......................... 121

$6 \mathrm{MAPbI}_{2.9-x} \mathrm{Br}_{x} C l_{0.1}$ Hybrid Halide Perovskites: Shedding Light on the Effect of Chloride and Bromide Ions on Structural and Photoluminescence Properties

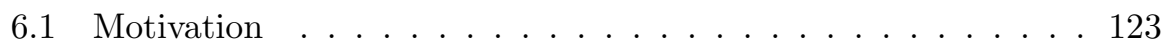

6.2 Experimental Procedure . . . . . . . . . . . . . . . 124

6.3 Results and Discussion . . . . . . . . . . . . . . 125

$6.3 .1 \quad X$-ray Analysis . . . . . . . . . . . . 125

6.3.2 FESEM Analysis . . . . . . . . . . . . . . 132

6.3.3 Absorbance ................... 133

6.3.4 Photoluminescence . . . . . . . . . . . 135

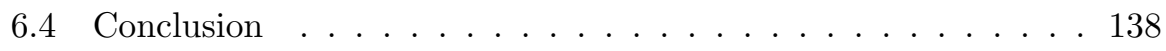

7 Impact of Iodide Substitution on the Physical Properties and Stability of Cesium Lead Halide Perovskite Thin Films $\mathrm{CsPbBr}_{3-x} I_{x}(0 \leq x \leq 1) \quad 139$

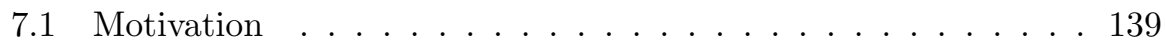

7.2 Experimental section . . . . . . . . . . . . . . . . 140 
7.3 Results and Discussion . . . . . . . . . . . . . . . . . . . . . 141

7.3 .1 X-ray Analysis . . . . . . . . . . . . . . . . . . 141

$7.3 .2 \quad$ FESEM Analysis . . . . . . . . . . . . . . . . 147

7.3.3 $U V$-vis Spectra and Luminescence Properties . . . . . 148

7.4 Conclusion . . . . . . . . . . . . . . . . . 153

8 Effect of Guanidinium on the Optical Properties and Structure of the Methylammonium Lead Halide Perovskite 155

8.1 Motivation . . . . . . . . . . . . . . . 155

8.2 Experimental procedure . . . . . . . . . . . . . . . 157

8.3 Results and Discussion . . . . . . . . . . . . . . . . 157

8.3.1 X-Ray Diffraction . . . . . . . . . . . . 157

$8.3 .2 \quad$ Absorbance . . . . . . . . . . . . . . . . 163

8.3.3 Photoluminescence . . . . . . . . . . . . . . . . . . 166

8.3.4 FESEM Analysis . . . . . . . . . . . . . . 170

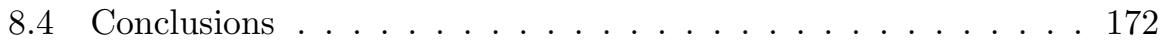

9 Improving Structural and Optical Properties via Imidazolium Cation Integration on Methylammonium Lead Iodide $\begin{array}{ll}\text { Perovskite } & 175\end{array}$

9.1 Motivation . . . . . . . . . . . . . . . 175

9.2 Experimental Procedure . . . . . . . . . . . . . . . . 177

9.2 .1 Perovskite Fabrication . . . . . . . . . . . . 177

9.3 Results and Discussion . . . . . . . . . . . . . . . . 178

9.3.1 X-ray Diffraction . . . . . . . . . . . . . . . . 178

$9.3 .2 \quad$ Absorbance . . . . . . . . . . . . . . . . . . . . . . 184

9.3.3 Photoluminescence . . . . . . . . . . . . . . . . . . 186

9.3.4 FESEM analysis . . . . . . . . . . . . . 188 
9.4 Conclusions .......................... 189

10 Conditions for Fabricating Efficient Devices with $\begin{array}{ll}\text { Methylammonium Lead Halide Perovskite } & 191\end{array}$

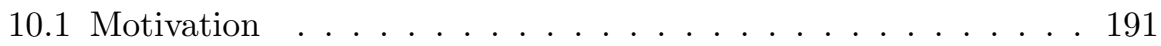

10.2 Experimental Procedure . . . . . . . . . . . . . 193

10.2.1 Materials and Precursor Solutions . . . . . . . . 193

10.2.2 Photovoltaic Devices Fabrication . . . . . . . . . 193

10.3 Results and Discussion . . . . . . . . . . . . . . 195

10.3.1 UV-vis Spectra and Photoluminescence . . . . . . . 195

10.3.2 FESEM analysis . . . . . . . . . . . . . 196

10.3.3 Current-Voltage Measurements . . . . . . . . . . 197

10.4 Conclusions ..................... . . 200

11 Conclusions $\quad 201$

$\begin{array}{ll}\text { Bibliography } & 204\end{array}$ 



\section{List of Figures}

$1.1 \mathrm{CO}_{2}$ level along the history. . . . . . . . . . . . 2

1.2 Annual and monthly mean global surface temperature anomalies. 3

$1.3 X$-rays stream off the Sun. . . . . . . . . . . . . . 6

1.4 Best PCEs for crystalline Si cells. . . . . . . . . . . . . 8

1.5 Prices of $S i$ solar cells. . . . . . . . . . . . . . . . . . 8

1.6 NREL efficiency chart. . . . . . . . . . . . . . . 11

2.1 Cubic, tetragonal and Orthorhombic phases of $M A P b I_{3}$ at different temperatures. . . . . . . . . . . . . . . 22

2.2 Bonding diagram of $\left(\mathrm{PbI}_{6}\right)^{-4} \ldots \ldots \ldots \ldots \ldots \ldots$

2.3 Comparison of aproximate $t$ and $\mu$ factors. . . . . . . . 36

2.4 Device architecture and cross-sectional FESEM image for a mesoporous oxide based perovskite solar cell. . . . . . . . . 43

$2.5 \quad A M 0$ spectrum compared with $A M 1.5$ spectrum. . . . . . . . 55

2.6 Schematic band diagram of a semiconductor. . . . . . . . 56

2.7 Shockley and Quisser theoretical maximum achievable $\eta$ for single-junction solar cells as a function of the bandgap. . . . . . 57

2.8 Schematic plot of a $p$ - $n$ solar cell junction and energy band level diagram. . . . . . . . . . . . . . . . . . 62

$2.9 \quad I-V$ curve representation with $V_{O C} \ldots \ldots \ldots \ldots \ldots$ 
2.10 $V_{O C}$ as function of bandgap for a cell with $A M 0$ and $A M 1.5$. . 67

2.11 Short-circuit current density as a function of the bandgap under $A M 0$ and $A M 1.5$ spectrum. . . . . . . . . . . . . 67

2.12 Graph of cell output current and power as a function of voltage. 69

2.13 Energy band diagram of a typical $M A P b I_{3}$ perovskite solar cell. 72

2.14 Example of a magnetic hysteresis loop for a ferroelectric material. 74

2.15 Hysteresis effect on a $J-V$ curve. . . . . . . . . . . . . . . . 75

4.1 X-Ray diffractograms of $\mathrm{MAPbX}_{3}(X=I, B r, C l)$ bulks. . . 89

4.2 Comparison of the powder and thin film $X$-ray diffractograms for $\mathrm{MAPbI}_{3}$. . . . . . . . . . . . . . . . . . . . . . . . . . . . 90

4.3 Comparison of the powder and thin film $X$-ray diffractograms

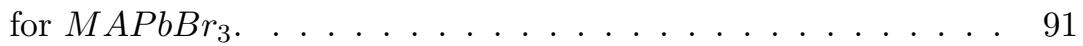

4.4 Comparison of the powder and thin film $X$-ray diffractograms for $\mathrm{MAPbCl}_{3}$. . . . . . . . . . . . . . . . . . 91

$4.5 X$-Ray diffractograms of $M A P b I_{3-x} B r_{x}$ films. . . . . . . . . . 92

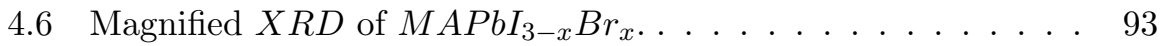

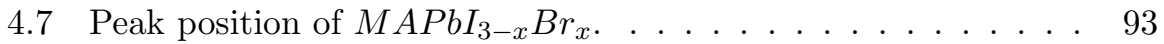

4.8 Lattice parameter of $\mathrm{MAPbI}_{3-x} \mathrm{Br}_{x} \ldots \ldots$. . . . . . . . . . . . 94

$4.9 X$-Ray diffractograms of $\mathrm{MAPbBr}_{3-x} \mathrm{Cl}_{x} \ldots \ldots \ldots$

4.10 Magnified $X R D$ of $M A P b B r_{3-x} C l_{x}$. . . . . . . . . . . . 95

4.11 Peak position of $\mathrm{MAPbBr}_{3-x} \mathrm{Cl}_{x}$. . . . . . . . . . . . . 96

4.12 Lattice parameter of $\mathrm{MAPbBr}_{3-x} \mathrm{Cl}_{x}$. . . . . . . . . . . . 96

4.13 Absorbance for $M A P b I_{3-x} B r_{x}$ and $M A P b B r_{3-x} C l_{x}$ thin films. 98

4.14PL spectra for $M A P b I_{3-x} B r_{x}$ and $M A P b B r_{3-x} C l_{x}$ thin film. 100

$4.15 P L$ spectra for $\mathrm{MAPbI}_{3}$ in cooling. . . . . . . . . . . . . . . 101

$4.16 P L$ spectra for $\mathrm{MAPbI}_{3}$ in heating. . . . . . . . . . . . . . . 102

$4.17 D S C$ plot $M A P b I_{3}$. . . . . . . . . . . . . . . . . 102 
$4.18 P L$ spectra for $M A P b B r_{3}$ in heating. . . . . . . . . . . . . . 103

$4.19 D S C$ plot of $M A P b B r_{3} \ldots \ldots \ldots 4$

$4.20 \mathrm{PL}$ spectra for $\mathrm{MAPbCl}_{3}$ in cooling. . . . . . . . . . . . . . . . 104

$4.21 D S C$ plot of $\mathrm{MAPbCl}_{3}$. . . . . . . . . . . . . . . . 105

4.22 FESEM images of $\mathrm{MAPbX}_{3}$. . . . . . . . . . . . . 106

5.1 XRD of $\mathrm{MAPbI}_{3-x} B r_{x}$ thin films. . . . . . . . . . . . . . . 112

$5.2 X R D$ of $M A P b I_{3-x} B r_{x}$ magnified in the region of the two strongest plane intensities. . . . . . . . . . . . . . . 113

5.3 Lattice parameters $M A P b I_{3-x} B r_{x}$ as a function of bromide content.

5.4 FESEM micrographs of thin films with different amount of bromide. .................... 115

5.5 AFM images of thin films with different amount of bromide. . . 116

$5.6 U V$-vis absorption spectra of $M A P b I_{3-x} B r_{x} \ldots \ldots 118$

5.7 Variation of the absorption edge with the chemical composition of bromide for $\mathrm{MAPbI}_{3-x} \mathrm{Br}_{x}$. . . . . . . . . . . . . 119

$5.8 P L$ spectra at room temperature for $M A P b I_{3-x} B r_{x} \ldots \ldots 120$

6.1 XRD patterns of $M A P b I_{3}$ and $M A P b I_{2.9-x} B r_{x} C l_{0.1}$. . . . 126

6.2 Magnified $X R D$ patterns of $M A P b I_{2.9-x} B r_{x} C l_{0.1}$. . . . . . . 127

6.3 Magnified $X R D$ of $\mathrm{MAPbI}_{2.2} \mathrm{Br}_{0.8}$ with and without a $10 \%$ of chloride. ....................... 128

6.4 Magnified $X R D$ of $M A P b I_{2.6} \mathrm{Br}_{0.4}$ with and without a $10 \%$ of chloride. . . . . . . . . . . . . . . . . 128

6.5 Deconvolution of $M A P b I_{2.6} \mathrm{Br}_{0.3} \mathrm{Cl}_{0.1}$ peak at 28.5 . . . . . . 129

6.6 Pawley fit of $M A P b I_{3}$ film. . . . . . . . . . . . . . . . 130

6.7 Pawley fit of $M A P b I_{2.4} B r_{0.5} C l_{0.1}$ film. . . . . . . . . . . . . 130

6.8 Pawley fit of $\mathrm{MAPbI}_{2.2} \mathrm{Br}_{0.7} \mathrm{Cl}_{0.1}$ film. . . . . . . . . . . . . 131 
6.9 Pawley fit of $M A P b I_{1.9} B r_{0.1} C l_{0.1}$ film. . . . . . . . . . . . 131

6.10 FESEM image of different bromide content. . . . . . . . . . . 133

$6.11 U V$-vis absorption spectra of $M A P b I_{2.9-x} B r_{x} C l_{0.1}$. . . . . . 134

$6.12 U V$-vis absorption spectra of $M A P b I_{2.6} B r_{0.4}$ and $M A P b I_{2.6} B r_{0.3} C l_{0.1} \ldots \ldots \ldots \ldots$

$6.13 P L$ spectra for $M A P b I_{3}$ and $M A P b I_{2.9-x} B r_{x} C l_{0.1}$. . . . . . 136

6.14 Comparison between the $P L$ emissions of $M A P b I_{2.6} B r_{0.4}$ and $M A P b I_{2.6} B r_{0.3} C l_{0.1} \ldots \ldots \ldots \ldots$

$7.1 X R D$ of $C s P b B r_{3-x} I_{x}(0 \leq x \leq 1) \ldots \ldots \ldots 14 \ldots$

7.2 Magnified $X R D$ of $C s \mathrm{PbBr}_{3-x} I_{x}$. . . . . . . . . . . . . . . 142

7.3 Pawley fit of the $\mathrm{CsPbBr}_{3}$. . . . . . . . . . . . . . . . 143

7.4 Pawley fit of the $C s \mathrm{PbBr}_{2.4} I_{0.6} \ldots \ldots \ldots$. . . . . . . . . 144

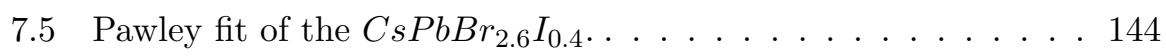

7.6 Pawley fit of the $\mathrm{CsPbBr}_{2} I \ldots \ldots \ldots$. . . . . . . . 145

7.7 Lattice parameters of $C s \mathrm{PbBr}_{3-x} I_{x}$. . . . . . . . . . . . 146

7.8 Dependence on $x$ of the $F W H M$ of the XRD pattern. . . . . . 147

7.9 FESEM micrographs of $\mathrm{CsPbBr} \mathrm{I}_{1}$ and $\mathrm{CsPbBr}_{3}$. . . . . . 148

$7.10 U V$-vis absorption spectra of $C s \mathrm{PbBr}_{3-x} I_{x} \ldots \ldots$. . . . . . . . . 149

7.11 Absorption edge for $\mathrm{CsPbBr}_{3-x} I_{x}$. . . . . . . . . . . . 150

$7.12 U V$-vis absorption and $P L$ spectra of $C s P b B r_{3}$. . . . . . . . 151

7.13 Absorbance spectra for $\mathrm{CsPbBr}_{2.8} I_{0.2}$ fresh, after aging and after annealing. . . . . . . . . . . . . . . . 152

7.14 Absorbance spectra for $\mathrm{CsPbBr}_{2.4} I_{0.6}$ fresh, after aging and after annealing. . . . . . . . . . . . . . . 152

7.15 Absorbance spectra for $\mathrm{CsPbBr}_{3}$ fresh, after aging and after annealing. ..................... 153

$8.1 X R D$ of $M A_{1-x} G A_{x} P b I_{3} \ldots \ldots \ldots \ldots$ 
8.2 Pawley refinement of the $X R D$ spectra for pure $G A P b I_{3}$. . . 159

8.3 XRD patterns of the $M A P b I_{3}$ and $M A_{1-x} G A_{x} P b I_{3}$. . . . 160

8.4 Lattice parameters as a function of $G A$ content. . . . . . . . . . 161

8.5 XRD for $\mathrm{MAPbI}_{3}$ fresh and after eight months. . . . . . . . . 162

8.6 XRD for $M A_{0.95} G A_{0.05} \mathrm{PbI}_{3}$ fresh and after eight months. . . . 162

8.7 $X R D$ for $M A_{0.90} G A_{0.1} P b I_{3}$ fresh and after eight months. . . . 163

8.8 $X R D$ for $M A_{0.85} G A_{0.15} \mathrm{PbI}_{3}$ fresh and after eight months. . . . 163

8.9 Thin film images and $U V$-vis absorption spectra of

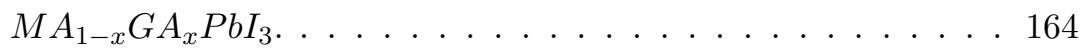

8.10 Tauc plots showing variations in optical bandgap. . . . . . . . . 165

8.11 Normalized $P L$ spectra of $M A_{1-x} G A_{x} P b I_{3} \ldots$. . . . . . . . . 166

$8.12 \mathrm{PL}$ spectra of $\mathrm{MAPbI}_{3}$ and $M A_{0.85} \mathrm{GA}_{0.15} \mathrm{PbI}_{3}$. . . . . . . . 167

8.13 $\mathrm{PL}$ spectra for $\mathrm{MAPbI}_{3}$ in heating at different temperatures. . 168

8.14 Magnified $P L$ spectra and the corresponding $F W H M$ value. . 168

8.15 Normalized $P L$ spectra for $M A_{0.85} G A_{0.15} P b I_{3}$ in heating. . . . 169

8.16 Magnified $P L$ spectra and $F W H M$ values for for $M A_{0.85} G A_{0.15} P b I_{3} \ldots \ldots \ldots$. . . . . . . . . . . . . . . . . . . 170

8.17 FESEM micrographs of $M A_{1-x} G A_{x} P b I_{3}$. . . . . . . . . . . . 171

$9.1 X R D$ for $M A_{1-x} I M_{x} P b I_{3}$. . . . . . . . . . . . . . . . 179

9.2 Magnified $X R D$ of $M A_{1-x} I M_{x} P b I_{3} \ldots \ldots \ldots$. . . . . . . . 180

9.3 Pawley refinement of $X R D$ for pure $I M P b I_{3} \ldots \ldots$. . . . . . . . 181

9.4 Pawley fit of $X R D$ patterns of the $M A P b I_{3}$ and $M A_{0.9} I M_{0.1} P_{b} I_{3} \ldots \ldots \ldots \ldots$

9.5 Composition-dependent evolution of lattice parameters as a function of $I M$ content. . . . . . . . . . . . . . . . . . . 183

9.6 Dependence of the $F W H M$ and the $X R D$ peak (100) on the $I M$ content. . . . . . . . . . . . . . . . . . . 184 
9.7 $U V$-vis absorption spectra of $M A_{1-x} I M_{x} P b I_{3}$ for different $I M$ contents. ..................... . . 185

9.8 Tauc plots of $M A_{1-x} I M_{x} \mathrm{PbI}_{3}$ showing variations in optical bandgap. ....................... 185

$9.9 P L$ spectra of $M A_{1-x} I M_{x} P b I_{3}$ for $x=0,0.2$. . . . . . . . 187

$9.10 P L$ spectrum and absorbance of $M A P b I_{3} \ldots \ldots$. . . . . . . . . . 188

$9.11 P L$ spectrum and absorbance of $M A_{0.8} I M_{0.2} P_{b I}$. . . . . . . 188

9.12 FESEM micrographs of $M A_{1-x} I M_{x} P_{b} I_{3}$. . . . . . . . . . . . 189

$10.1 P L$ spectrum and absorbance of $M A P b I_{3}$ thin film. . . . . . . 195

10.2 Cross-section FESEM image of the device and a device structure picture. . . . . . . . . . . . . . 196

$10.3 J-V$ curve corresponding to the $A 8$ device. . . . . . . . . . . . . 197

$10.4 J-V$ curve corresponding to the $A 8$ device before and after spiro-OMeTAD oxidation. . . . . . . . . . . . . . 199 


\section{Abbreviations and Symbols}

$t \quad$ tolerance factor

$\mu \quad$ octahedral factor

$g \quad$ molecular factor

$\tau \quad$ new tolerance factor

$\eta_{C} \quad$ collection efficiency

Voc open-circuit voltage

Isc short-circuit current

$F F \quad$ fill factor

$\eta \quad$ efficiency

c speed of the light

$E_{\lambda} \quad$ photon energy

$J \quad$ current density-voltage

$w_{n} \quad$ width of the depletion region

voc normalized voltage

$P C E$ power conversion efficiency

$\eta_{C}^{e x t} \quad$ external collection efficiency

$\eta_{C}^{i n t} \quad$ internal collection efficiency

$\eta_{B C} \quad$ maximum efficiency

$D \quad$ crystallite size

$R w p \quad$ weighted profile factor

$R p \quad$ profile factor

I light intensity

$T \quad$ transmittance

$\alpha \quad$ absorption coefficient

$E g \quad$ absorption edge 



\section{Chapter 1}

\section{Introduction}

\subsection{Global Energy Crisis}

Ever since its creation, the Earth's climate has been changing. Currently, there is scientific evidence of seven glacial advance and retreat cycles in the last 700,000 years. The last ice age ended approximately 7, 000 years ago, bringing about the modern climate era and human civilization. Most climate change is attributed to the small changes in the amount of solar energy received, as a result of small variations in the Earth's orbit. With $95 \%$ certainty, current global warming is the result of human industrial activity and their need for energy since the mid-20th century, evolving at an unprecedented rate [1-5].

First demonstrated in the mid-19th century, carbon dioxide $\left(\mathrm{CO}_{2}\right)$ and other gases were shown to trap heat nature, and, nowadays, there is consensus among the scientific community that increased levels of greenhouse gases must have caused the Earth become warmer in response. Indeed, ice cores extracted from Greenland, Antarctica, and tropical mountain glaciers have shown that the Earth's climate reacts to changes in greenhouse gas levels. Historical evidence found in tree rings, ocean sediments, coral reefs, and layers of sedimentary rocks, reveals current warming is occurring about ten times 
faster than the average rate of warming in ice-age-recovery [6].

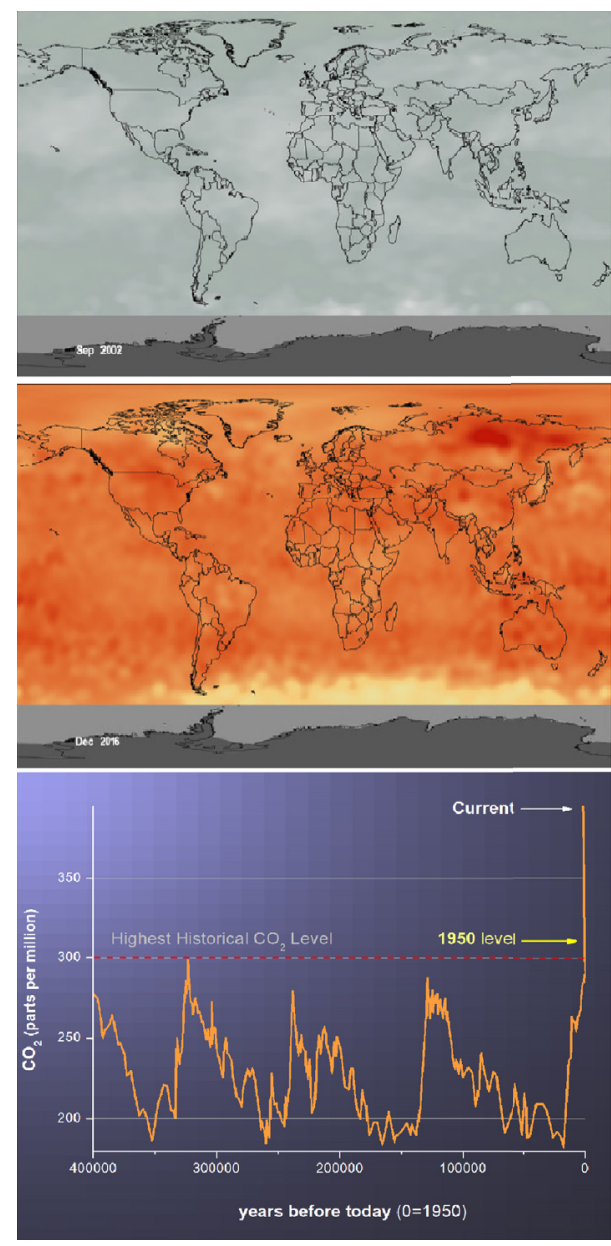

Figure 1.1: Level of $\mathrm{CO}_{2}$ (parts per million) along history, based on the comparison of atmospheric samples contained in ice cores and more recent direct measurements. Since the Industrial Revolution, $\mathrm{CO}_{2}$ level has surpassed 300 ppm (Credit: Vostok ice core data/J.R. Petit et al.; NOAA Mauna Loa $\mathrm{CO}_{2}$ record) [7].

Largely due to increased carbon dioxide and other manmade emissions into the atmosphere, since the 19th century, the planet's average surface temperature has risen by about $1.1^{\circ} \mathrm{C}$ [8]. This radiative effect from $\mathrm{CO}_{2}$ and other greenhouse gas emissions is projected to increase the average global 
surface temperature between 1.0 and $3.7^{\circ} C$ by 2100 , compared to that of the late 20 th century.
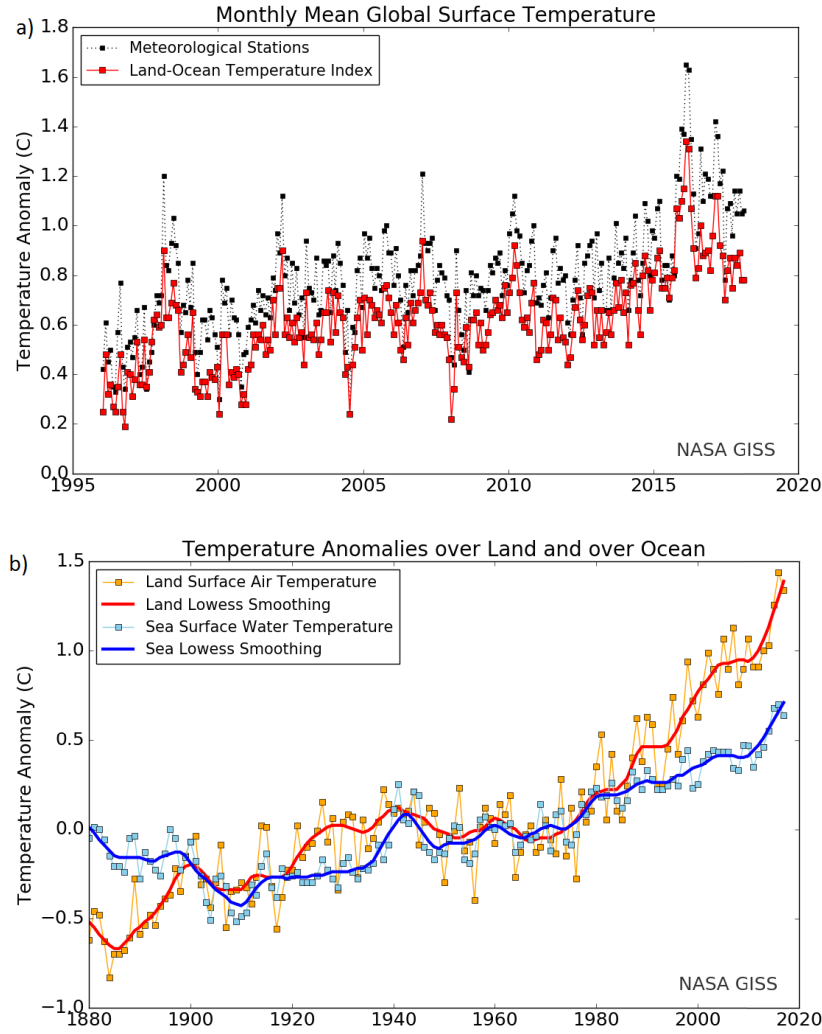

Figure 1.2: a) Monthly mean global surface temperature anomalies vs. 1951-1980. The black dotted line shows estimates based on meteorological station data only; the red line shows estimates that additionally use ocean temperature data from ships and buoys. b) Annual (thin lines) and five-year lowess smooth (thick lines) for the temperature anomalies averaged over the Earths land area and sea surface temperature anomalies averaged over the part of the ocean that is free of ice at all times (open ocean). Images courtesy of NASA CIGS [9].

At the end of 2015, 195 nations signed the Paris Agreement [10] with the aim of limiting the temperature rise to well below $2.0^{\circ} \mathrm{C}$ by the end of the century and therefore, avoiding the worst impacts of climate change. Reaching this objective will require huge reductions in greenhouse gas emissions. 
Growing worldwide energy demand, expected to increase by $47 \%$ from 2010 to 2035 [11], one of the most serious problems facing the world today, is one of the main causes for this increase in temperature. So much so, that the United Nations has recently cited it as one of the top sustainable development targets for the next 15 years [12]. Burning of fossil fuels for energy generation produces around 21.3 billion tones of $\mathrm{CO}_{2}$ per year [13], although it is estimated that natural processes absorb about half of that amount, still there is a net increase of 10.65 billion tones of atmospheric $\mathrm{CO}_{2}$ per year, contributing to global warming [14]. Aside from the significant global dependence on fossil fuels, their finiteness is a problem which must urgently be addressed before a global energy crisis occurs. Energy can also be related to other broader issues, whether that be the availability of food and clean drinking water, the destruction of ecosystems, economic growth, conflict or climate change, the production, distribution and usage of energy is fundamental [14].

A global movement towards the generation of renewable energy is underway to help reduce global greenhouse gas emissions. Renewable energy sources may be the key to reach the ambitious objective of reducing greenhouse gas emissions, and among them, solar energy stands out for the large magnitude of energy available. The amount of solar irradiation incident on the earth in one hour exceeds current annual global energy demand [15], making photovoltaic devices a compelling alternative to fossil fuel-based power generation. There is a variety of methods for potentially achieving independence from fossil fuels, each with a number of technical challenges that will require a great deal of research and development before commercially viable solutions can be implemented. It is quite likely that several technologies will have to be synchronized in order to achieve continuous and uninterrupted energy supply, and this is likely to depend upon the specific geographical location. 


\subsection{The Case for Solar Power}

The fundamental driving force behind almost any natural processes on Earth is the Sun. The Sun (Figure 1.3) is a ball of gas composed of $70.6 \%$ hydrogen and $27.4 \%$ helium. Its enormous mass is held together by gravitational attraction, producing immense pressure and temperatures about 15 million degrees Celsius, enough to sustain thermonuclear fusion. This is a process in which atoms combine to form larger atoms and in the process release huge amounts of energy. Specifically, in the Sun's core, hydrogen atoms fuse to make helium. The energy produced in the core powers the Sun and produces all the heat and light the Sun emits.

The huge magnitude of energy it provides make solar energy a suitable candidate source from which to extract energy and convert it into a usable form. The most direct approach is to convert incident light energy to electricity, using photovoltaics. Apart from requiring lower arable land usage, less infrastructure and a reduced visual impact, it can be applied in developing regions of the world, where other techniques are not as feasible.

Solar energy technology allows to generate electricity at the point of use, with solar panels, and, therefore, minimizing losses associated with long range distribution of power. However, there are still many challenges to overcome with solar energy technology, such as avoiding potential shortfalls and storage problems. Nevertheless, it is clear that solar power has the theoretical potential to supply much of the world's energy demand [16]. In fact, only relatively small areas with solar panels would be necessary to produce enough electricity to satisfy the global demand. In practice, distribution losses would force solar technology to produce this power as close as possible to where it is needed, but it, nevertheless, highlights the potential of solar power. Furthermore, the continued improvement of power conversion efficiency will 
help reduce the area required for solar cells, as well as help to bring down costs on a price per unit of energy basis.

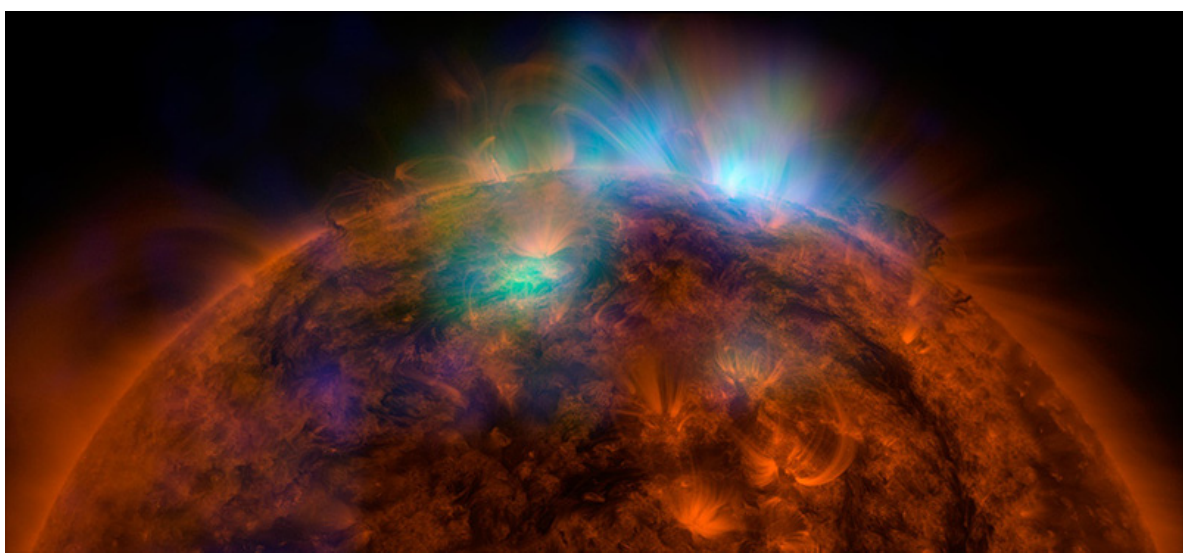

Figure 1.3: $X$-rays stream off the sun in this image showing observations from by NASA's Nuclear Spectroscopic Telescope Array, or NuSTAR, overlaid on a picture taken by NASA's Solar Dynamics Observatory (SDO) [17]. Image credit: NASA/JPL-Caltech/GSFC

\subsection{Solar Cells}

A solar cell or photovoltaic solar cell can be defined as a device capable of absorbing sunlight and converting it into electricity. They are based on the photovoltaic effect $(P V)$ that was experimentally demonstrated by Edmond Becquerel in 1839. In that experiment, silver chloride was placed in an acidic solution and illuminated while connected to platinum electrodes, generating voltage and current. At the end of 19th century, in 1873, Willoughby Smith et al. first described a $P V$ effect in selenium. Ten years later, in 1883, Charles E. Fritts built the first solid state photovoltaic cell by placing a sheet of amorphous selenium on a metal backing and covering the selenium with a transparent gold film, but with a conversion efficiency less than 1\%. Later, in 1954, Chapin et al. [18] at Bell Labs discovered and demonstrated the first 
silicon single-crystal solar cell that reached $6 \%$ efficiency. Solar cells gained prominence with their incorporation into the 1958 Vanguard I satellite.

\section{Solar cell history}

Up to now, solar cells can be classified in three different generations. They have evolved using different light-harvesting material components and mechanisms to convert light into charge carriers.

The first generation of solar cells was based on crystalline silicon, still dominating technological applications of photovoltaics due to their high power conversion efficiency $(P C E)$ and stability. The second generation was based on amorphous silicon, copper indium gallium selenide $(C I G S)$, or cadmium telluride $(C d T e)$. They had lower conversion efficiencies than the first generation but were cheaper. The third generation is based on organic materials and intended to break the Shockley-Queisser limit, although none have actually achieved this breakthrough.

First-generation solar cells The first generation of solar cells was based on crystalline silicon, developed initially with the interest of its use in the US-American space program. The standard device structure of a silicon solar cell is a $p$ - $n$ junction, formed from an $n$-type and a $p$-type doped silicon region.

Silicon-based solar cells have reached a high power conversion efficiency of $26.1 \%$ [19] for single crystal (non-concentrator) and a long-term stability, and have become a fundamental pillar in the solar energy market. Furthermore, this status will continue in the near future since it is very difficult for new technologies to compete due to the rapid fall in the cost of silicon $P V$ (Figure 1.5)(from around $\$ 2.00 /$ watt in 2010 , to approximately $\$ 0.25$ / watt in 2017 ) $[20]$. 


\section{Best Cell Efficiencies for Cristalline Si Cells}

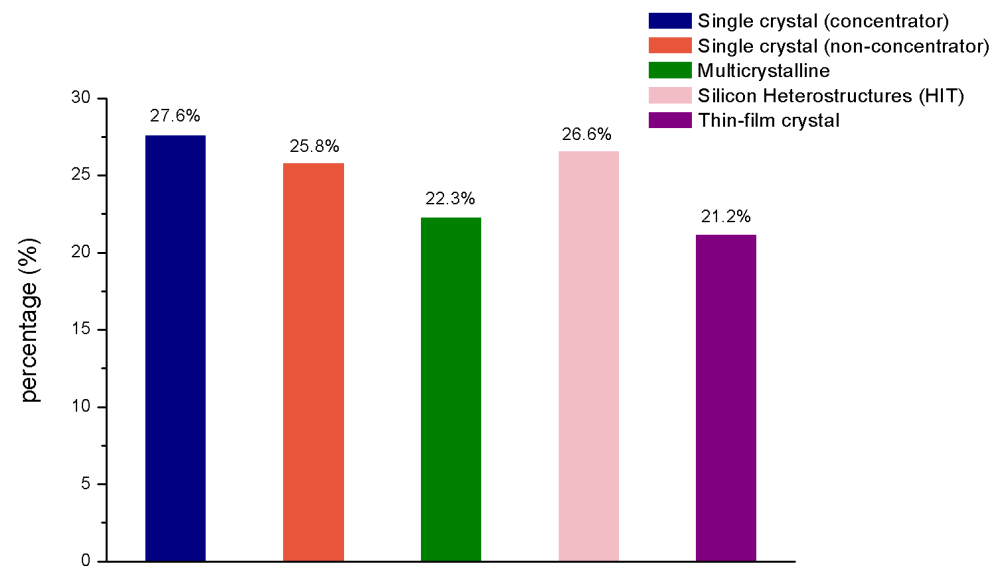

Figure 1.4: Best PCEs for crystalline Si cells. Data obtained form NREL [19].

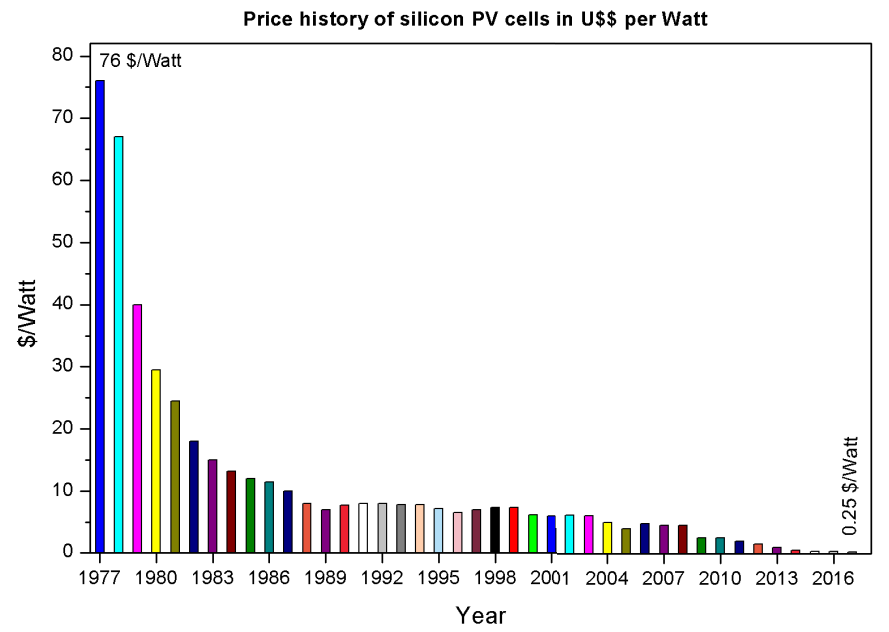

Figure 1.5: Prices of $S i$ solar cells along the last years. Data obtained form [20] and Bloomberg New Energy Finance \& PV.energytrend.com.

Despite their high efficiencies, crystalline silicon solar cells require high-grade silicon and an energy intensive manufacturing process. Since they apply well to rooftop applications where area is a limiting factor, the high cost is offset by the need for maximum power production. When area is 
not a limiting factor, like solar farms, it has been demonstrated that it is more cost-effective to use cheaper panels with lower efficiencies, for example, multicrystalline silicon solar cells, with $22.3 \%$ efficiency and being at least $50 \%$ cheaper. Recently, researchers have focused on new silicon based technologies like silicon heterostructures (HIT) or thin film crystals reaching efficiencies of $26.6 \%$ and $21.2 \%$, respectively [19].

Second-generation solar cells One of the main problems of the use of silicon in solar cells is its indirect bandgap and its consequent low absorption coefficient, being necessary layers in the order of about $100 \mu \mathrm{m}$ to obtain enough light absorption. Second-generation solar cells appeared as an alternative to minimize the quantity of material used. These solar cells have an inorganic light-absorbing layer with a high extinction coefficient so that a thin film is enough for light absorption, and are therefore also known as thin film solar cells. These cells employ new materials with good photovoltaic properties in thin films being about $1 \mu \mathrm{m}$ thick, such as amorphous silicon, cadmium telluride $(C d T e)$, cadmium sulfide $(C d S)$, copper-indium-gallium-selenide $(C I G S)$ and copper-zinc-tin-sulfide/selenide $(C Z T S)[21,22]$.

Cadmium telluride photovoltaics constitute the second largest commercial technology after silicon, due to its structural simplicity [23] and have reached a record-cell efficiency of $22.7 \%$ [19] on a glass substrate. Through a vacuum-based method, the conductive glass substrate is covered by an $n$-type layer of $C d S$ and then a $p$-type layer of $C d T e$. The final layer is a back contact, usually aluminium $(A l)$ [24]. In addition, the bandgap for the $C d T e$ is $1.5 \mathrm{eV}$, very close to the optimum bandgap for $p$ - $n$ junction solar cells due to their black body limit [25]. One of the main advantages of employing these solar cells is that $C d T e$ has a temperature coefficient about half that of the one of silicon, thus significantly reducing the relative efficiency loss. In warm climates 
with high solar irradiances $C d T e$-based solar cells are potentially more efficient than silicon alternatives.

On the other hand, CIGS solar cells have reached an efficiency of $22.6 \%$ [19]. In order to manufacture a standard CIGS solar cell, a glass substrate is covered by a back contact molybdenum layer followed by a copper, (indium, gallium) selenide (sulfur) light-absorbing layer. A cadmium sulfide buffer layer is then deposited. Finally, the solar cell is completed by a layer of zinc oxide $(Z n O)$ and/or zinc oxide-aluminum on the front surface [26, 27]. Vacuum-based techniques are employed to create these solar cells. The CIGS absorbing layer is produced through two processes. In the one-step method, all materials $(C I G S)$ are deposited in a single step by using various evaporation processes. In the two-step process, the copper, indium and gallium are first deposited, followed by the sulfurization or selenization process [21].

Third-generation solar cells The definition of third-generation solar cells is wide, encompassing a significant range of different technologies. Usually it refers to organic, quantum dot and dye-sensitized solar cells $(D S S C)$ but also include multi-junction solar cells. Only limited commercial success has been achieved with third-generation solar cells, with dye sensitized solar cells perhaps having the most success up to date. Nevertheless, promising alternatives are beginning to appear.

Dye-sensitized solar cells, created in the early 1990s, operate via a biomimetic process similar to photosynthesis $[14,28,29]$. Visible light is absorbed by dye molecules, sensitizing a wide-bandgap semiconductor. In order to enhance light absorption, the semiconductor is found in the form of mesoporous titanium dioxide $\left(\mathrm{TiO}_{2}\right)$, being only $10 \mu \mathrm{m}$ thick, to provide a high surface area for dye molecules to be adsorbed on its surface. Most common dyes are ruthenium-based metal complexes, adsorbed through carboxylate 
groups. When illuminated, excited electrons in the dye are injected into the conduction band of the mesoporous $\mathrm{TiO}_{2}$, diffusing then to the electrode contact. Oxidized dye molecules regenerated by a redox electrolyte, commonly in the form of an iodide-triiodide redox couple in an organic solvent. Record efficiencies have been obtained recently using cobalt $(I I I / I I)$ redox complexes with more favorable redox potentials and therefore higher open-circuit voltages [30]. Dye cells usually contain a dense $\mathrm{TiO}_{2}$ layer underneath the mesoporous layer to act as a blocking layer to recombine electrons in the FTO (Fluorine doped Tin Oxide) substrate with triiodide in the electrolyte [14].

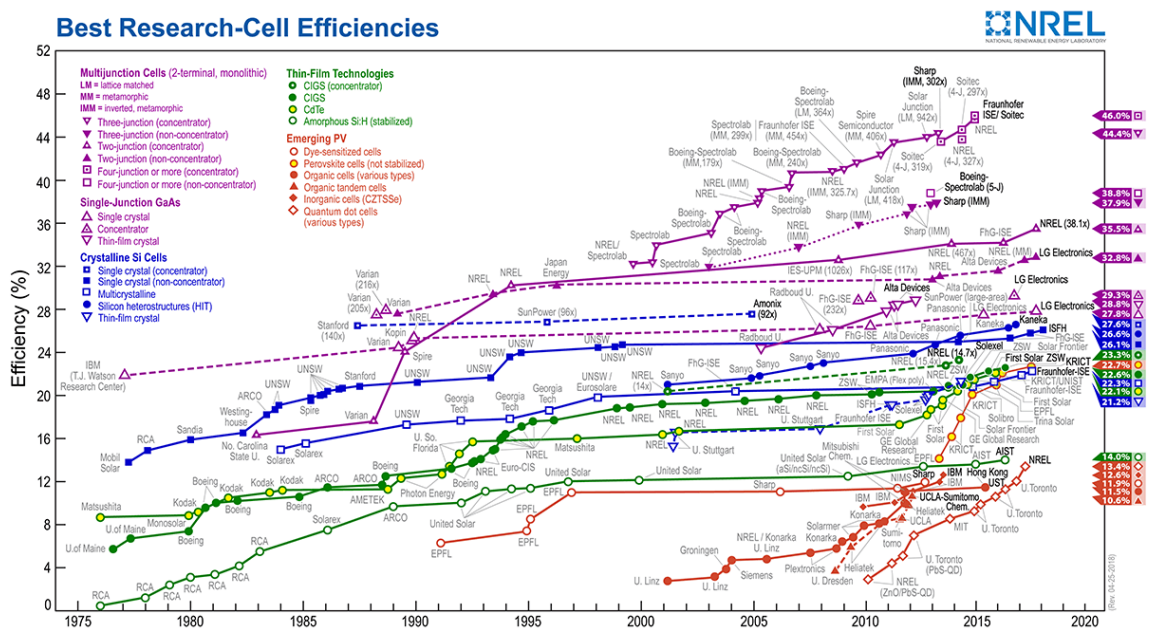

Figure 1.6: Efficiency Chart of best research-cell efficiencies of different technologies. The fast improvement of perovskite solar cells in the last decade can be appreciated. Courtesy of NREL [19].

DSSC device efficiencies have reached $11.9 \%$ [19,31], having a number of advantages such as low-cost production, abundant materials, roll-to-roll processing on flexible substrates, and good response in diffuse light conditions. However, the main scale-up limiting factor is their instability, associated with the use of a liquid electrolyte. Redox species are corrosive to sealing materials, and the volatile solvent is prone to leaking. Water or a humid atmosphere can 
also significantly affect performance. A number of attempts have been made to employ a solid state electrolyte, i.e., a hole transport material to reduce the dye molecules before they degrade. The best results have been obtained using a heavily doped organic hole-conducting material, spiro-OMeTAD [32]. However, device efficiencies have so far failed to exceed $8 \%$, mainly due to the thickness of the titania layer that limits light absorption [33].

Finally, perovskite solar cells, born in the third generation, are one of the most promising new solar technologies in decades. These devices are made from low-cost materials, and their low temperature fabrication processes allow them to be used in many applications. Additionally, the $P C E$ of these devices is close to that of market-leading multi-crystalline silicon technology. The high efficiency of these devices is driven by the relatively low bandgap of methylammonium (MA) lead iodide and a low loss-in potential (the difference between the bandgap energy and the device open-circuit voltage). Nowadays, PCE of single-junction perovskite solar cells has reached 22.7\% Figure 1.6 shows the best efficiencies for different PV technologies. Further improvements are expected to come from future reductions in the loss-in potential, which can be done by decreasing the density of trap states that act as charge recombination centers in a device. The following chapter is devoted to perovskite solar cells and compositional engineering. 


\section{Chapter 2}

\section{Organic-Inorganic Halide Perovskites}

\subsection{Organic-Inorganic Hybrid Materials}

Organic-inorganic hybrid $(O I H)$ materials constitute a huge family of materials with different structures, based on inorganic and organic components that have attracted the attention of researchers due to their potential application in technology. On the one hand, some pure organic materials have good conductive and light-emitting properties, with low-cost processing methods [34], but lack the long-term stability to be fully scalable and applicable. On the other hand, inorganic materials have also remarkable properties like, for example, high, medium and low conductivity and long-term stability. By synthesizing organic-inorganic hybrid materials, attempts are made to combine properties which are typical of inorganic and organic substances in one material.

Photovoltaics is one of the fields of research that has benefited significantly from these OIH materials. Hybrid systems can simultaneously use 
efficient organic absorbers and the efficient dielectric screening of inorganic components. This, in turn, facilitates dissociation into free charge carriers at the respective organic/inorganic side and transport to the respective electrode.

The quantity of each organic/inorganic material affects the interface structures, the corresponding optoelectronic properties, excitation lifetimes and charge-carrier transport properties of the hybrid. In order to adjust the desirable properties of an $O I H$ material, it is necessary to know the binding strength of electron-hole pairs, their spatial extension, the nature of exciton diffusion, and the time scales of their formation and recombination.

$O I H$ materials structural lattice comprises sequentially repeating units of organic and inorganic parts, merging or enhancing chemical, physical or structural properties of the materials through different bonding interactions (e.g., covalent, ionic bonding, Van der Waals).

In recent years several alternative materials have been tested [35-41], obtaining notable photo-electrochemical and physical properties such as a tunable band-gap, high conductivity and thermal stability [34,41]. These properties have been successfully applied in optoelectronic devices such as solar cells or light-emitting diodes (LEDs) [42].

\subsection{Perovskites for Solar Cells}

\subsubsection{Perovskite Material}

Perovskite is a mineral with the formula $\mathrm{CaTiO}_{3}$ discovered in 1839 by the Prussian mineralogist Gustav Rose and named after the Russian mineralogist Lev Aleksevich von Perovski. Natural crystals have a hardness between 5.5 and 6 and a density of $4000-4300 \mathrm{kgm}^{-3}$ with an orthorhombic crystal structure. Perovskite has given its name to an important subfamily of organic-inorganic hybrid materials of compounds called perovskites, with the general formula 
$A B X_{3}$, where $A$ is normally a large cation, $B$ is a medium-sized cation and $X$ is an anion, with an electrically neutral overall ionic structure [43].

Since the discovery of the dielectric and ferroelectric properties of barium titanate, $\mathrm{BaTiO}_{3}$, and its applications in electronics in the 1940s, the importance of perovskites has been growing exponentially. Nowadays, many compounds adopting the perovskite structure are known. Subsequent attempts to improve the material properties have led to a great deal of research on the structure, with the result of vast numbers of new phases with very useful physical and chemical properties, far broader than those shown by $\mathrm{BaTiO}_{3}$ (see Table 2.1). Examples of insulating, semiconducting and superconducting perovskite materials are known. These materials are the archetypal systems for phases transitions with accessible cubic, tetragonal, orthorhombic, trigonal and monoclinic polymorphs depending on the tilting and rotation of the $B X_{3}$ polyhedra in the lattice.

Within the formal stoichiometry of $A B X_{3}$, charge balancing $\left(q_{A}+q_{B}+\right.$ $\left.3 q_{X}=0\right)$ can be reached in different ways. For metal oxide perovskites $\left(\mathrm{ABO}_{3}\right)$, some example are $\mathrm{KTaO}_{3}, \mathrm{SrTiO}_{3}$ and $\mathrm{GdFeO}_{3}$. The range of accessible materials can be extended by partial substitution on the anion sublattice. With substitution on the metal sublattice, double, triple and quadruple perovskites can be formed [44].

For halide perovskites, the oxidation states of the two cations must sum three, so the only viable ternary combination is, for example, $\mathrm{CsSnI}_{3}$. In hybrid halide perovskites such as $\mathrm{CH}_{3} \mathrm{NH}_{3} \mathrm{PbI}_{3}$, also denoted as $\mathrm{MAPbI}_{3}$, a divalent inorganic cation is present and the monovalent metal is replaced by an organic cation of equal charge. Any molecular cation can be used, if there is sufficient space to fit it within the cavity, otherwise the three-dimensional perovskite network is broken. Currently, the cations 
for high-efficiency photovoltaic solar cells are $R b, C s$, methylammonium $(M A)$, and/or formamidinium $(F A)$. Molecules larger than $F A$, such as ethylammonium $(E A)$, guanidinium $(G A)$, and imidazolium $(I A)$ have been investigated, obtaining suitable candidates for multication $3 D$ perovskites with unexpected properties such as suppressing halide segregation and stability [45]. For layered structures, the crystal properties become anisotropic with larger carrier masses and stronger exciton binding energies.

Taking into account the formal stoichiometry $A B X_{3}$, the ideal cubic structure takes place when the $B-X-B$ angle is $180^{\circ}$, although several adjustments may occur to the octahedral units resulting in a tilted angle and lower symmetry. The connectivity of the octahedral units can be affected by the size of the cation and the functionality between the cation and the metal, leading to a corner-sharing, face-sharing, or edge-sharing structure [40]. Face-sharing and edge-sharing structures lead to non-perovskite lattices. However, several lattices can be formed through the connectivity of the structural octahedral units:

- a three-dimensional $(3 D)$ perovskite network is created when the connectivity is present along all three axes

- a two-dimensional $(2 D)$ perovskite lattice is obtained when the connectivity is lost in one axis. The general formula for $2 \mathrm{D}$ materials can be $A_{2} M X_{4}$ or $A_{2}^{+} M X_{4}$.

- a one-dimensional $(1 D)$ perovskite lattice is obtained when the connectivity is lost in two axis. The general formula for materials can be $A_{3}^{+} M X_{5}$ or $A_{3} M X_{5}$

The flexibility of the perovskite framework allows to include cations such as $\mathrm{NH}_{4}^{+}$, which can be considered spherical at normal temperatures. More complex phases, such as the inorganic-organic hybrid compounds $\mathrm{MAPbX}_{3}$, 
where $X$ is typically $C l, B r, I$ or a combination of these anions, have been synthesized. Nevertheless, flexibility comes at a structural cost. The $A B X_{3}$ perovskite structure depends on structural variations such as composition, temperature and pressure, all of which strongly affect physical properties.

Table 2.1: Representative $A B X_{3}$ perovskite phases.

\begin{tabular}{|c|c|c|c|c|c|}
\hline \multirow[t]{2}{*}{ Phase } & & \multirow[t]{2}{*}{ Space group } & \multicolumn{3}{|c|}{ Unit cell } \\
\hline & & & $a(n m)$ & $b(n m)$ & $c(n m)$ \\
\hline \multicolumn{6}{|l|}{1,2} \\
\hline & $A g M g F_{3}$ & $C, P m \overline{3} m(221)$ & 0.41162 & 0.41162 & 0.41162 \\
\hline & $\mathrm{CsPbI}_{3}$ & $C, P m \overline{3} m(221)$ & 0.62894 & 0.62894 & 0.62894 \\
\hline & $K C u F_{3}$ & $T, I 4 / m c m(140)$ & 0.56086 & 0.56086 & 0.76281 \\
\hline & $\mathrm{NaMgF}_{3}$ & O, Pbnm (62) & 0.48904 & 0.52022 & 0.71403 \\
\hline
\end{tabular}

1,5

$\begin{array}{lllll}\mathrm{KTaO}_{3} & \mathrm{C}, \mathrm{Pm} \overline{3} m(221) & 0.40316 & 0.40316 & 0.40316 \\ \mathrm{KNbO}_{3} & \mathrm{O}, \mathrm{Amm} 2(38) & 0.3971 & 0.5697 & 0.5723\end{array}$

2,4

$\begin{array}{lllll}\mathrm{SrTiO}_{3} & \mathrm{C}, \mathrm{Pm} \overline{3} m(221) & 0.3905 & 0.3905 & 0.3905 \\ \mathrm{BaTiO}_{3} & \mathrm{~T}, \mathrm{P} 4 m m(99) & 0.39906 & 0.39906 & 0.40278 \\ \mathrm{CaTiO}_{3} & \mathrm{O}, \mathrm{Pbmn}(62) & 0.54035 & 0.54878 & 0.76626 \\ (\mathrm{Fe}, \mathrm{Mg}) \mathrm{SiO}_{3} & \text { O, Pnma (62) } & 0.5020 & 0.6900 & 0.4810\end{array}$

3,3

$\begin{array}{lllll}\mathrm{BiFeO}_{3} & \mathrm{Tr}, \mathrm{R3c}(161) & 0.55798 & 0.55798 & 1.3867 \\ \mathrm{BiInO}_{3} & \mathrm{O}, \text { Pnma (62) } & 0.59546 & 0.83864 & 0.50619 \\ \mathrm{ErCoO}_{3} & \mathrm{O}, \text { Pbnm (62) } & 0.51212 & 0.54191 & 0.73519 \\ \mathrm{GdFeO}_{3} & \mathrm{O}, \mathrm{Pbnm}(62) & 0.53490 & 0.56089 & 0.76687\end{array}$

4, 5
$\operatorname{ThTaN}_{3}$
C, $P m \overline{3} m$
0.4020
0.4020
0.4020 


\subsubsection{Perovskite-based Solar Cells}

Organometallic perovskite materials have been studied since 2007 [42], and their were first used in solar cells in 2009 [14, 46], when Kojima et al. demonstrated the use of methylammonium lead iodide and bromide as sensitizers in a dye cell with a mesoporous titania thickness of $10 \mu \mathrm{m}$ and a liquid iodide-triiodide electrolyte. The perovskite was deposited onto $\mathrm{TiO}_{2}$ by spin coating a $D M F$ solution containing methylammonium halide and lead iodide. The initial PCE of $2.19 \%$ was rapidly improved modifying mesoporous titanium oxide and the electrolyte nature, obtaining conversion efficiencies of $3.13 \%$ and $3.81 \%$ for $M A P b B r_{3}$ and $M A P b I_{3}$ perovskites, respectively. Also, a remarkable voltage of open circuit $\left(V_{O C}\right)$ of $0.96 \mathrm{~V}$ for $\mathrm{MAPbBr}_{3}$ was achieved. However, it was noted that cells stopped to operate because of perovskite solving in acetonitrile, and it was, therefore, replaced with the nonpolar solvent ethylacetate [47] obtaining improved efficiencies of $6.2 \%$. Nevertheless, cells lost $80 \%$ of their efficiency after $10 \mathrm{~min}$.

The use of quantum dots $(Q D)$, such as $C d S, C d S e, P b S, P b S e, \operatorname{InP}$, In $A s$ and $S b_{2} S_{3}$, as absorbers instead of dyes significantly boosted the research on solar cell devices [47].

An inorganic perovskite, $\mathrm{CsSnI}_{3}$, has been used as the hole transport material in dye cells incorporating the common ruthenium sensitizer $N 719$. The device configuration was identical to that used in liquid dye cells, but with the electrolyte replaced with the solid state perovskite. A device efficiency of 8.51\% was recorded, which exceeds all other efficiencies for solid state dye cells achieved [48]. Despite this promising result, the study of tin-based perovskites has been hampered due to their instability [40].

The first use of a methylammonium lead halide perovskite in a solid state cell was reported in 2012, and since then, its performance has been steadily 
improved. As a result, different device architectures have been reported with high performance.

The perovskite can act as an efficient light absorber as the mesoporous layer thickness is reduced to a few hundred nanometers. The sensitization of $\mathrm{TiO}_{2}$ with $\mathrm{MAPbI}_{3}$ using spiro-OMeTAD $\left(2,2^{\prime}, 7,7^{\prime}\right.$ - tetrakis (N, N-dip-methoxypheniamine) - 9,9' - spirobifluorene) as hole transporter, resulted in an efficiency of $9.7 \%$ with an operability of about 500h [49]. By photoinduced absorption spectroscopy, two processes - injection of holes from perovskite to spiro-OMeTAD and electrons to mesoporous $\mathrm{TiO}_{2}$ were found. Interestingly, very good results were obtained when employing the same configuration but without the hole transport material, reaching an efficiency of $5.5 \%$ [50]. This proved that a perovskite is capable of transporting holes in addition to acting as a sensitizer. Recently, Lee et al. achieved efficient performance without a $\mathrm{TiO}_{2}$ mesoporous layer [51]. Furthermore, mixed perovskite $\mathrm{M} \mathrm{APbI}_{2} \mathrm{Cl}$ was shown to have simultaneously the properties of an absorber and conductor of electrons. Using spiro-OMeTAD as a hole transporter and mesoporous $\mathrm{Al}_{2} \mathrm{O}_{3}$ (an insulator with the bandgap between 7 and $9 \mathrm{eV}$ ) instead of titanium oxide, an efficiency of $10.9 \%$ was obtained. Such cells are called mesosuperstructured cells. Sequential deposition also became an important improvement when Grätzel et al. reported an efficiency of $14.1 \%$ using this method [52]. This procedure introduces lead iodide into a mesoporous $\mathrm{TiO}_{2}$ film, then dipped into a dilute methylammonium iodide $(M A I)$ solution forming the perovskite. The increase in performance can be related to the improved morphology when forming the perovskite within the pores.

Since a perovskite has a very high absorption coefficient, it does not need an increased surface area of a mesoporous structure, though a high film coverage 
is necessary. As the spin coating method may lead to numerous pin-holes in the thin film, thus, increasing the probability of a short-circuit, other methods have been employed. Some of them are revised in this thesis, such as evaporation of the perovskite precursor materials [53]. As a consequence, $M A P b I_{3-x} C l_{x}$ planar devices have reached efficiencies of $15.4 \%$.

The use of the chloride perovskite, produced by mixing $\mathrm{MAI}$ and $\mathrm{PbCl}_{2}$ in a 3:1 molar ratio as the perovskite precursor solution, initially achieved better performance than for the iodide alone, formed from equimolar mixing of $M A I$ and $\mathrm{PbI}_{2}$. Nevertheless, actual research questions these achievements, since the effect of $\mathrm{Cl}$ doping is not well defined, and it is not incorporated into the perovskite lattice [54], unlike bromide ions [55]. No significant change has been found in the bandgap of a iodide perovskite and a chloride incorporated perovskite, although chloride seems to be related to the improved perovskite crystallization process rather than an actual doping effect [54].

Since vacuum deposition is a complicated technological process, Chen et al. [56] proposed a similar approach [57] in which instead of dipping, the lead iodide film moves through vapors of methylammonium iodide, leading to the formation of a film with an optimal coverage of the dense $\mathrm{TiO}_{2}$ in the planar configuration of the cell. With this technology, an efficiency exceeding $12 \%$ was obtained. Recently, a three-step technology [58] that allows the exclusion of a film of non-reacted $\mathrm{PbI}_{2}$ has been reported.

The modification of dense titanium oxide in the planar configuration of cells with small amounts of graphene [59] and titanium diisopropoxide bis (acetilacetonate) [60] to decrease series resistance has obtained conversion efficiencies of $15.6 \%$ and $15.9 \%$, respectively. Coordination of the energy levels of the hole-transport component (poly - triarylamine - PTAA) [61] and spiro - OMeTAD [62] as well as optimization of series resistance has enhanced 
conversion efficiency from $16 \%$ to $19.3 \%$ [63].

More information about deposition methods and compositional engineering is shown in this chapter.

\subsubsection{Crystal Structure}

This section describes the ideal $A B X_{3}$ perovskite structure and some structural variations affecting the chemical and physical properties. Weber first reported the cubic perovskite crystal structure of $\mathrm{MAPbI}_{3}$ [64], but, since the molecular cations are orientationally disordered in the crystal, an effective average higher lattice symmetry takes place [65]. Recently, three phases of $\mathrm{MAPbI}_{3}$ depending on the temperature, have been reported: orthorhombic, tetragonal and cubic Bravais lattices [66] (see Figure 2.1). X-ray diffraction is able to distinguish the position of Bragg peaks between the three phases, but the peak intensities arising from $\mathrm{CH}_{3} \mathrm{NH}_{3}^{+}$relative to $\mathrm{PbI}_{2}^{-}$are too weak to assign accurate molecular orientations.

- Orthorhombic Phase $(T<165 K)$

The $\mathrm{MAPbI}_{3}$ orthorhombic perovskite maintains its structural stability up to about $165 K$, with an initially assigned Pna21 spatial group [67], and reassigned to Pnma via higher quality powder neutron diffraction data. The structure is a $\sqrt{2} a \times \sqrt{2} a \times 2 a$ supercell expansion of the simple cubic perovskite lattice.

The $\mathrm{CH}_{3} \mathrm{NH}_{3}^{+}$sublattice is fully ordered but the ordering may be sensitive to the material preparation and/or cooling rate into this phase. It is possible that different ordering might be frozen into the low-temperature phase.

- Tetragonal Phase $(165-327 K)$

At $165 \mathrm{~K}, \mathrm{MAPbI}_{3}$ changes from orthorhombic to tetragonal space group $I 4 / m c m$ [67]. This can be also considered a $\sqrt{2} a \times \sqrt{2} a \times 2 a$ expansion of 
the cubic perovskite unit cell. The molecular cations are no longer in a fixed position.

- Cubic Phase $(T>327 K)$

As the temperature is raised, tetragonal lattice parameters become more isotropic. A transition to a cubic phase occurs around $327 K$, which can be seen from changes in the heat capacity, as well as in temperature-dependent neutron diffraction [68]. The cubic space group has been assigned the space group $\operatorname{Pr} \overline{3} m$. Nevertheless, the local structure will necessarily have a lower symmetry. Indeed, for the bromide and chloride analogues of $\mathrm{MAPbI}_{3}$, pair-distribution function analysis of X-ray scattering data indicates a local structure with significant distortion of the lead halide framework at room temperature [69].

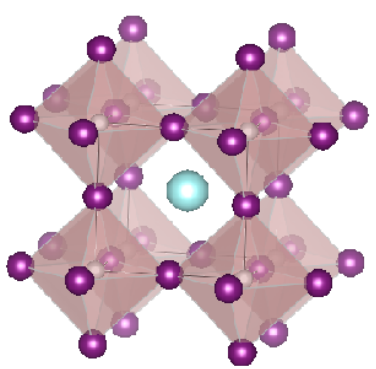

Cubic

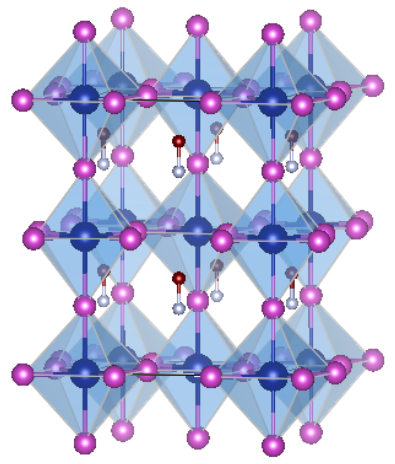

Tetragonal

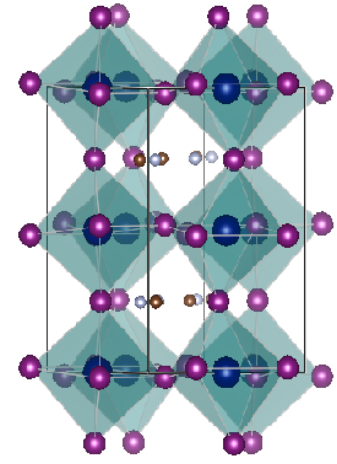

Orthorhombic

Figure 2.1: Cubic, tetragonal and Orthorhombic phases of $\mathrm{MAPbI}_{3}$ at different temperatures.

\subsubsection{Stability and Compositional Engineering}

One of the main disadvantages of the use of perovskite solar cells is their low long-term stability. Usually, high performance PSCs lose their efficiencies 
and degrade in a significantly short time interval, ranging from several minutes to some days. In order to become fully scalable, PSCs should meet at least stability standards of thin film photovoltaic cells (IEC 61646) in which only a $10 \%$ decrease from the initial performance is allowed over a period of $1000 h$ in accelerated aging tests (only $5 \%$ is allowed for crystalline silicon solar cells).

The performance of a solar cell is typically expressed in terms of its efficiency $(\eta)$, calculated from the current-voltage $(I-V)$ parameters, the short-circuit current density $\left(J_{S C}\right)$, the open-circuit voltage $\left(V_{O C}\right)$ and the fill factor $(F F)$. These parameters reflect any degradation of the solar cell, although they do not provide much information about its mechanisms. To study the degradation mechanisms in PSCs, measurement techniques such as current voltage measurement $(I-V)$ under 1 Sun lighting condition, electrochemical impedance spectroscopy $(E I S)$, incident photon-to-collected-electron efficiency (IPCE) technique, Raman and FTIR spectroscopy [70,71], spatially-resolved photocurrent, and intensity modulated photovoltage spectroscopy, among others, should be applied. The EIS and IPCE techniques are usually helpful to figure out which component of the cell suffers degradation, although sometimes, due to the overlap of the time-constants of different interfaces, it is difficult to differentiate the degrading components. Finally, to gain insight into chemical and structural changes, techniques such as SEM, TEM, AFM, Raman, $P L$ and $X R D$ are often applied.

Manufacturing methods of PSCs affect both their performance and stability. Nevertheless, many stability improvements have been made, thanks to the fast progress in light absorber materials syntheses, device fabrication procedures and the better understanding of the degradation mechanisms. For example, Chen et al. [72] reported an efficient PSC with a high stability 
(>90\% of the initial PCE) in a light soaking test for $1000 \mathrm{~h}$.

In the study of degradation mechanisms, stability of PSCs is categorized into intrinsic and extrinsic types. Intrinsic stability includes chemical and structural stability over a range of photovoltaic operating conditions (humidity, temperature and light exposure) in the presence of a certain amount of impurities, especially oxygen and water, which are introduced in the device during manufacturing. On the other hand, extrinsic stability deals with the failures of sealing and moisture blocking layers.

The optoelectronic properties such as bandgap, absorption and charge carrier mobilities of perovskite material depend on its structure. Therefore, any crystal distortion due to phase transformation can affect these properties, degrading the performance of PSCs. Also, both oxygen and water above certain amounts can degrade perovskites. For example, an excess of oxygen can result in oxidation of the organic components in the cell. Polar solvent and highly hygroscopic nature of the amine salts can also degrade the perovskite materials. Finally, the photo-oxidation process is unavoidable in most semiconducting materials.

On the other hand, an important advantage of perovskites is their flexibility in composition, with each site of the $A B X_{3}$ crystal structure easily replaceable by several other elements and/or compounds. Compositional engineering of the perovskite is one strategy to improve device efficiency and has become the main source of efficiency and stability improvements. Some of the results obtained in the literature are presented in this section in terms of the $A-, B$-, and $X$-site ion replaced by suitable alternatives. 


\section{Anion $X$ Alternatives}

Methylammonium lead iodide $\left(\mathrm{MAPbI}_{3}\right)$, the most commonly studied perovskite solar cell material, has been widely characterized as chemically unstable when exposed to moisture, heat, and light [73,74]. This instability translates to $P C E$ degradation rates above $0.1 \% h^{-1}$ for some $\mathrm{MAPbI}_{3}$ perovskite solar cells. It has also been reported that $M A P b I_{3}$ undergoes a transformation from the tetragonal to the cubic phase at around $53^{\circ} \mathrm{C}$, affecting the photovoltaic performance causing thermal instability. However, several studies have shown that $\mathrm{MAPbI}_{3}$ can still exist as tetragonal phase even after heating to $100^{\circ} \mathrm{C}[40,49,51,75]$. Thermal instability may originate either from intrinsic instability of the perovskite film or the HTM layer.

It has been demonstrated that a small addition of $\mathrm{Br}^{-}$in the $\mathrm{MAPbI}_{3}$ was able to stabilize and improve the photovoltaic properties. It has also been found that the $E_{g}$ could be tuned from $1.57 \mathrm{eV}$ for the pure $\mathrm{MAPbI}_{3}$ to $2.28 \mathrm{eV}$ for the pure $\mathrm{MAPbBr} r_{3}$ by controlling the $I / B r$ ratio of the perovskite precursor solution [55]. Unfortunately, the $M A P b I_{3-x} B r_{x}$ perovskite, for $0.2<x<0.85$, segregates into separate iodine and bromine rich domains when exposed to light.

Small additions of chloride have also been studied. It has been reported that an excess of $\mathrm{Cl}^{-}$to the solution, employing $\mathrm{MACl}$, retards crystallization of $\mathrm{MAPbI}_{3}$ [51]. First reports on the structure of $\mathrm{MAPbI}_{3-x} \mathrm{Cl}_{x}$ claimed to exhibit enhanced properties, but later results showed that chloride addition primarily acted as a crystallization retardant, facilitating the formation of bigger grains and improving uniformity. It has been demonstrated, experimentally as well as theoretically, that the formation of continuous solid phase $\mathrm{MAPbI}_{3-x} \mathrm{Cl}_{x}$ is actually not alloyed and that chloride incorporation into $\mathrm{MAPbI}_{3}$ is possible only at a relatively low concentration, so that it could 
be classified as a dopant agent rather than alloy, as can be seen in this thesis.

Several different non-halide anions, also called pseudo-halides, have been tested for formation of perovskite materials, such as borohydride $\left(\mathrm{BH}_{4}^{-}\right)$ and thiocyanate $\left(S C N^{-}\right)[76]$. However, pure organic lead borohydrides or thiocyanate perovskites have not been successfully synthesized.

\section{Cation $A$ Alternatives}

Since the bandgap of the perovskite-structured formamidinium lead iodide $\left(\mathrm{FAPbI}_{3}\right)$ is narrower $(1.48 \mathrm{eV})$ than that of $M A P b I_{3}(1.53 \mathrm{eV})$, it is possible to replace $M A$ with $F A$ and achieve higher photocurrents. $F A P b I_{3}$ undergoes phase transformation to tetragonal crystalline structure at $150^{\circ} \mathrm{C}$ [77], showing larger thermal stability as compared to $M A P b I_{3}$ substrate [78]. The main disadvantage is that pure $F A P b I_{3}$ is very sensitive to moisture and degrades rapidly at room temperature from the $\alpha$ to $\delta$ phase, which has a wider band gap and a non-perovskite structure [40]. Therefore, one of the important goals of compositional engineering is the stabilization of the $\alpha-F A P b I_{3}$ phase.

Initially, $\mathrm{FAPbI}_{3}$ was mixed with $15 \mathrm{~mol} \%$ of either $\mathrm{MAPbI}_{3}$ [79], $\mathrm{MAPbBr}_{3}$ [80] or $\mathrm{CsPbI}_{3}$ [81] obtaining significantly improved results on stability. However, the $\mathrm{FA}_{1-x} \mathrm{MA}_{x} \mathrm{PbI}_{3-y} \mathrm{Br}_{y}$ perovskite composition has shown the highest efficiency with $F A: M A$ and $I: B r$ ratios of 2:1 and 5:1, respectively [82]. It is worth noting that the majority of devices showing $>20 \%$ PCE have used this perovskite composition or small modifications $[82,83]$.

The thermal instability of perovskite materials with organic cations has prompted interest in inorganic perovskite materials for solar cell applications. Among other suitable properties, inorganic cations do not evaporate as easily as organic cations and show greater stability under the working conditions of 
solar cells.

$\mathrm{Cs}^{+}$was the first inorganic cation to be introduced into the lead halide perovskite for manufacturing new photoabsorbing solar cell materials [84]. Cesium has been introduced into the structure of $\mathrm{FAPbI}_{3}$ stabilizing the perovskite structure at room temperature and increasing the $P C E$ [85]. The cubic phase of $\mathrm{CsPbI}_{3}$ is not stable at room temperature, just like $\mathrm{FAPbI}_{3}$ but, $C s F A$ alloys yield a stable cubic perovskite phase. The cubic phase of $\mathrm{CsPbI}_{3}$ has an $E_{g}$ of $1.73 \mathrm{eV}$, ideal for tandem applications with $c-S i$. Conversely, the $\alpha$-phase of $\mathrm{CsPbBr}_{3}$ is stable at room temperature but its $E_{g}$ $(2.36 \mathrm{eV})$ is far too large to yield a $P C E$ of more than $15 \%$ in single-junction.

Other examples of compositional engineering have looked at the use of different $A$-site cations entirely. Some reports have studied the use of guanidinium $(G A)$ cations, given the similar structure to $F A$. Some characteristics and properties of $G A$-addition in PCSs are studied herein. Also, other cations such as ethylammonium $(E A)$ and imidazolium $(I M)$ have been investigated in the literature, and, particularly, the incorporation of $I M$ is studied in this thesis. Multiple-cation perovskite absorbers have also been studied. Saliba et al. showed that adding $C s I$ to the $F A_{1-x} M A_{x} \mathrm{PbI}_{3-y} \mathrm{Br}_{y}$ perovskite improved device efficiency, stability, and reproducibility [83]. One reason for this is the small size of $\mathrm{Cs}^{+}$, that makes the cubic structure more favorable [81]. Additionally, the $\alpha$ phases of $\mathrm{FAPbI}_{3}$ and $\mathrm{CsPbI}_{3}$ are similar in terms of their atomistic structure and unit cell volume.

Finally, it is worth noting that devices made with 5-aminovaleric acid (5- $A V A)$ mixed with $M A$ have been reported to be most stable under illumination [86]. Indeed, perovskites comprising 5- $A V A / M A$ cations fully retained their PCEs for over one year under constant illumination in ambient air [87]. 


\section{Lead Alternatives}

Despite the appealing advantages of $\mathrm{Pb}$-based perovskites, such as intensive broad-band absorption, high charge carrier mobility and long charge diffusion length [49], their stability in ambient environment, intrinsic toxicity and anomalous hysteresis $[88,89]$ are still challenges to overcome. Indeed, the high toxicity of lead has made the European Union's Restriction of Hazardous Substances directive to prohibit its use in any electrical or electronic equipment $[90,91]$. Therefore, numerous researchers have focused on designing new lead-free perovskites as efficient as lead-based perovskites. It has been demonstrated that the outstanding photovoltaic properties of lead-based perovskites were not only attributed to the high symmetry of structure, but also to the strong $\mathrm{Pb} 6 s-I 5 p$ anti-bonding coupling. The lowest unoccupied states consist of $\mathrm{Pb} 6 p-I 5 s \sigma$-antibonding and $\mathrm{Pb} 6 p-I 5 p \pi$-antibonding orbitals. The higher occupied states can be decomposed into three parts (see Figure 2.2):

- a $\mathrm{Pb} 6 s-I 5 p \sigma$-antibonding orbital in the top of the states,

- $I 5 p$ orbitals in the middle energy region and

- $\mathrm{Pb} 6 p-I 5 s$-bonding and $\mathrm{Pb} 6 p-I 5 p \pi$-bonding orbitals in the bottom of the state.

The $\mathrm{Pb} 6 s-I 5 p \sigma$-bonding orbital is situated in the lower energy region. It has been proved that that the top valence band and bottom conduction band of $\mathrm{MAPbI}_{3}$ and $2 \mathrm{D}$ butylammonium lead iodide, $\left(\mathrm{C}_{4} \mathrm{H}_{9} \mathrm{NH}_{3}\right)_{2} \mathrm{PbI}_{4}$, are composed primarily of $\sigma$-antibonding stated of $\mathrm{Pb} 6 s$ and $I 5 p$ orbitals and $\sigma$-antibonding states of $\mathrm{Pb} 6 p$ and $I 5 s$ orbitals, respectively [92]. 


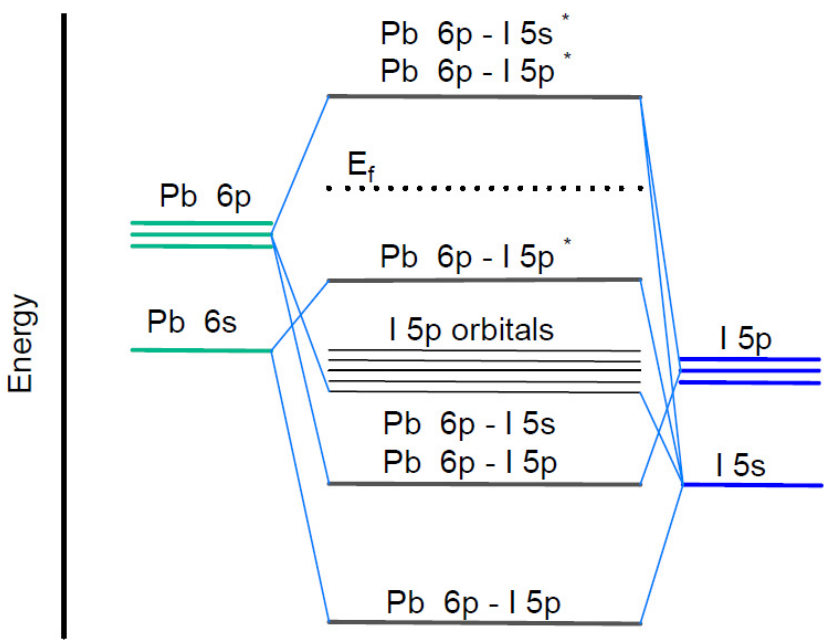

Figure 2.2: Bonding diagram of $\left(\mathrm{PbI}_{6}\right)^{-4}$ cluster (zero-dimensional system).

Metal cations of $S n^{2+}, G e^{2+}, B i^{3+}$, and $S b^{3+}$, which have $n s^{2}$ lone pairs, and can form an octahedral structure with halogen anions, can be candidates for lead substitution $[93,94]$. Depending on the level of lead substitution, we have two different types of perovskites: fully lead-substituted perovskites and partially lead-substituted perovskites.

Pure-tin-halide perovskites $\left(M A S n X_{3}, F A S n X_{3}\right.$ and $\left.C s S n X_{3}\right)$ have been studied in parallel with the study of lead halide perovskites [95]. Of all the closest elements to $\mathrm{Pb}$ in the group $\mathrm{IV}$ in the periodic table with lower toxicity, tin $(S n)$ is considered the most promising alternative. $S n^{2+}$ has a similar radius as $P b^{2+}(P b 1.49 \AA$ and $S n 1.35 \AA$ ), so no obvious lattice distortion will be caused after replacing/partly substituting for lead in perovskites [96]. Nevertheless, the toxicity issue has not been completely solved. It has been demonstrated that $S n$ is more easily cleared away from the human body (around one year) than $\mathrm{Pb}$ (20 years), but the intrinsic instability of $S n$-containing perovskites may result in decomposition into unstable products 
$\mathrm{SnI}_{2}$, and further decomposition into $H I$ along with toxicologically inactive oxygenated $S n$ precipitates [91]. This problem can be mitigated by enhancing the stability of $S n$-based perovskite solar cells.

A $\mathrm{MASnI}_{3}$ perovskite with a $P C E$ of around $6.4 \%$ has been reported for use in solar cells $[97,98]$. However, the use of $S n$ presents its own drawbacks, since the +2 oxidation state of $S n$, required to form a perovskite, is not stable and the metal rapidly oxidizes to the +4 state upon exposure to oxygen or humidity in the air. This means that any exposure to air can cause immediate oxidation of tin making the manufacturing process difficult at best. Structural and electrical properties, such as charge transport and recombination, doping densities, carrier lifetimes, mobilities and diffusion length for pure-tin-halide perovskites have been extensively studied $[99,100]$. For the $M A S n I_{3}$, a direct bandgap of $1 \mathrm{eV}-1.3 \mathrm{eV}$ is in good agreement with the experimental values [101]. However, it has been found that the oxidation of $S n^{2+}$ to $S n^{4+}$ can be slowed down replacing the $M A^{+}$organic cation by $\mathrm{FA}^{+} . \mathrm{FASnI}_{3}$ has a more suitable bandgap of $1.41 \mathrm{eV}$ and a single stable phase over a broad temperature of up to $200^{\circ} \mathrm{C}$.

$\mathrm{Cs}^{+}$is another frequently used inorganic $A$ cation. It has been demonstrated that $C s S n X_{3}$ possesses higher hole mobility, and lower exciton binding energy $(0.1$ or $18 \mathrm{meV})$ than the conventional $\mathrm{MAPbI}_{3}(30 \mathrm{meV})$ [99]. In addition, the $3 D$-orthorhombic $C s S n I_{3}$ perovskite is a $p$-type semiconductor with favorable bandgap of $1.3 \mathrm{eV}$ and high optical absorption coefficient $\left(10^{4} \mathrm{~cm}^{-1}\right.$ comparable to $\left.\mathrm{MAPbI}_{3}\right)$ [99]. However, the $\mathrm{CsSnX} \mathrm{X}_{3}$ has been demonstrated to be a unique temperature-dependent phase-transition material, exhibiting four polymorphs [102,103]. The $C s S n I_{3}$ perovskite device has reached a PCE of $13 \%$ [104], with transitions being caused by $\mathrm{Cs}^{+}$cation migration and the plasticity of the $S n-X-S n$ angle. 
Other metal halide perovskites such as $A G e X_{3}, A_{3} B i_{2} X_{9}$, and $A_{3} S b_{2} X_{9}$, or hybrid binary metal double perovskites such as $\mathrm{Cs}_{2} \mathrm{BiAgBr}_{6}, \mathrm{Cs}_{2} \mathrm{BiAgCl}_{6}$, and $\mathrm{Cs}_{2} \mathrm{InAgCl}_{6}$ have been studied in depth in order to avoid or mitigate the stability problems, but up to know, none of them have reached an efficiency high enough to compete with the lead-based perovskite.

Another solution is to partially replace the lead element to prepare PSCs of low-toxic without sacrificing efficiency. The $S n-P b$ hybrid perovskite solar cell $(M A / F A) P b_{1-x} S n_{x} X_{3}(x \leq 1)$ seems to be a compromise when considering the efficiency and lead-toxicity at the same time [105]. Alloyed 50/50 Sn/Pb halide perovskites have reached a 13.6\% PCE [106] and, recently, a $17.6 \%$ efficiency was obtained for the devices having a $40 \% \mathrm{~Pb}$ content. The reasons should be explained by the following points:

(i) $\mathrm{Pb}^{2+}$ is a stable state and further attributes the $\mathrm{Pb}^{2+}$-based perovskites to form a highly symmetrical $3 D$ structure.

(ii) The unique electronic features with strong $\mathrm{Pb} 6 s-I 5 p$ antibonding coupling would help to form a $3 D$ electronical state.

Additionally, rapid crystallization of $S n$-based perovskite films also affects the quality of the films. Therefore, additives such as $S n F_{2}$, that inhibit the oxidation reaction and additional solvent engineering that control film growth rate are widely reported. However, excessive $S n F_{2}$ induces phase separation of perovskite thin films.

Based on the extensive research done, it has been proved that no $\mathrm{Pb}$-free perovskites can compete in $P C E$ with pure- $P b$-based perovskites. Fortunately, the highest efficiency of the $S n-P b$ hybrid solar cell was achieved with $\mathrm{Pb}$-poor content, which is expected to further reduce lead usage in the future. The application of low-bandgap $S n-P b$ hybrid perovskites as bottom cell absorbers in tandem cells looks very promising, indeed since the highest 
efficiency of all-perovskite tandem cell reached $21.2 \%$, which is competitive with perovskite-silicon and perovskite-CIGS tandem cells. However, despite the toxicity of lead, large-scale manufacture of lead halide perovskite solar cells might not be as bad as it appears.

Table 2.2 Effective radii of several relevant ions and cations used and proposed for synthesis of perovskite materials for solar cell purposes.

\begin{tabular}{|c|c|c|c|c|c|}
\hline Cation $A$ & $\begin{array}{c}\text { Effective } \\
\text { radius } \\
r_{A, \text { eff } / p m}\end{array}$ & Cation B & $\begin{array}{c}\text { Effective } \\
\text { radius } \\
r_{\text {A, effipm }}\end{array}$ & Anion $X$ & $\begin{array}{c}\text { Effective } \\
\text { radius } \\
r_{A, \text { eff } / \mathrm{pm}}\end{array}$ \\
\hline Ammonium, $\left[\mathrm{NH}_{4}\right]^{+}$ & 146 & $\mathrm{~Pb}^{2+}$ & 119 & Chloride, $\mathrm{Cl}^{-}$ & 181 \\
\hline Hydroxylammonium, $\left[\mathrm{NH}_{3} \mathrm{OH}\right]^{+}$ & 216 & $\mathrm{Sn}^{2+}$ & 110 & Bromide, $\mathrm{Br}^{-}$ & 196 \\
\hline Methylammonium, $\left[\mathrm{CH}_{3} \mathrm{NH}_{3}\right]^{+}$ & 217 & $B i^{3+}$ & 103 & lodide, I- & 220 \\
\hline Hydrazinium, $\left[\mathrm{NH}_{3} \mathrm{NH}_{2}\right]^{+}$ & 217 & $\mathrm{Sb}^{3+}$ & 76 & Thiocyanate, $\mathrm{SCN}^{-}$ & 220 \\
\hline Azetidinium, $\left[\left(\mathrm{CH}_{2}\right)_{3} \mathrm{NH}_{2}\right]^{+}$ & 250 & $\mathrm{Ge}^{2+}$ & 73 & Borohydride, $\mathrm{BH}^{4-}$ & 207 \\
\hline Formamidinium, $\left[\mathrm{CH}\left(\mathrm{NH}_{2}\right)_{2}\right]^{+}$ & 253 & & & & \\
\hline Imidazolium, $\left[\mathrm{C}_{3} \mathrm{~N}_{2} \mathrm{H}_{5}\right]^{+}$ & 258 & & & & \\
\hline Dimethylammonium, $\left[\left(\mathrm{CH}_{3}\right)_{2} \mathrm{NH}_{2}\right]^{+}$ & 272 & & & & \\
\hline Ethylammonium, $\left[\left(\mathrm{CH}_{3} \mathrm{CH}_{2}\right) \mathrm{NH}_{3}\right]^{+}$ & 274 & & & & \\
\hline Guanidinium, $\left[\left(\mathrm{NH}_{2}\right)_{3} \mathrm{C}\right]^{+}$ & 278 & & & & \\
\hline $\mathrm{K}^{+}$ & 164 & & & & \\
\hline $\mathrm{Rb}^{+}$ & 172 & & & & \\
\hline $\mathrm{Cs}^{+}$ & 188 & & & & \\
\hline
\end{tabular}

It should be noted that the optimal perovskite layer thickness in the $P S C$ is around $400 \mathrm{~nm}$, so the quantity of lead employed may be small enough to compensate for its presence with a positive environmental impact. This leads to a popular comparison within the perovskite community: if the lead from a single lead-acid car-battery were to be completely converted to be use in $P S C s$, an area of approximately $7000 \mathrm{~m}^{2}$ could be covered with perovskite 
solar cells [107]. Furthermore, the issue of lead exposure could be overcome by proper encapsulation and recycling of lead-based PSCs.

\section{The Goldschmidt Tolerance Factor}

Perovskite instability has caused the field of research to be expanded to explore new types of perovskites. Predicting crystal structure from chemical composition is actually an important challenge in the discovery of new materials. An important tool when studying the suitability of new materials to form a perovskite structure is the Goldschmidt tolerance factor, which has been recently updated and improved. From a crystallographic perspective, the ideal perovskite unit cell has no adjustable atomic position parameters, so that any compositional change must be accommodated by a change in the lattice parameter, simply a sum of anion and cation bond lengths. The cubic unit cell edge, $a$, verifies

$$
a=2(B-X)
$$

where $B-X$ means the bond length. The width of the cuboctahedral cage site, $\sqrt{2} a$, is equal to twice the $A-X$ bond length, i.e.,

$$
\sqrt{2} a=2(A-X)
$$

This means that the ideal structure is formed when

$$
\frac{(A-X)}{(B-X)}=\sqrt{2}
$$

or

$$
\frac{(A-X)}{\sqrt{2}(B-X)}=1
$$

This relationship was employed by Goldschmidt in 1926, in order to 
predict the probability that a pair of ions would form a perovskite structure phase. By the time Goldschmidt presented the relationship, only a few crystal structures had been determined, so ionic radii were used as a substitute for measured bond lengths. Table 2.2 shows the radii of several relevant ions and cations used and proposed for synthesis of perovskite materials for solar cell applications. It is assumed that in order to form a stable structure, the cations only need to touch the surrounding anions (Goldschmidt's rule), then:

$$
\frac{\left(r_{A}+r_{X}\right)}{\left(r_{B}+r_{X}\right)}=\sqrt{2}
$$

or

$$
t=\frac{\left(r_{A}+r_{X}\right)}{\sqrt{2}\left(r_{B}+r_{X}\right)}=1
$$

where $t$ is called the tolerance factor, $r_{A}$ is the radius of the cation, $r_{B}$ is the radius of the octahedrally coordinated cation and $r_{X}$ is the radius of the anion. Despite the simplicity of the tolerance factor, it has had a significantly predictive power, especially for oxides, where ionic radii are known with greatest precision. A tolerance factor close to 1 would mean a high probability of formation of a perovskite structure. It has been found that if $t$ has a value between 0.9 and 1 , a cubic perovskite structure is probable. If $t>1$, meaning a larger $A$ and smaller $B$, a hexagonal packing of the $A X_{3}$ layers is preferred. When $t$ is between 0.71 and 0.9 , the structure distorts to the cuboctahedral coordination polyhedron. For lower values of $t, A$ and $B$ cations are of similar size and are associated with the ilmenite structure, $\mathrm{FeTiO}_{3}$. In the case of chlorides and sulphides, the tolerance factor is lower than that for oxides and fluorides, so that cubic and distorted cubic phases form for values 0.8 and 0.9 . Hexagonal perovskites form if $t$ is greater than 0.9 .

The tolerance factor can be extended to more complex compositions 
employing an average value for ionic radii or bond length. For example, for an $A$-site substituted phase $A_{1-x} A_{x}^{\prime} B X_{3}$

$$
t=\frac{\left[(1-x) r_{A}+x r_{A^{\prime}}+r_{X}\right]}{\sqrt{2}\left(r_{B}+r_{X}\right)}
$$

and in the case of $B$-site substitution $A B_{1-x} B_{x}^{\prime} X_{3}$

$$
t=\frac{\left(r_{A}+r_{X}\right)}{\sqrt{2}\left[(1-x) r_{B}+x r_{B^{\prime}}+r_{X}\right]}
$$

Apart from the tolerance factor, the perovskite stability is defined by an octahedral factor, $\mu$, defined by

$$
\mu=\frac{r_{B}}{r_{X}}
$$

$\mu$ should take values between 0.442 and 0.895 . The crystalline structure and the values of the parameters $t$ and $\mu$ for more frequently used perovskites $M A P b I_{3}, M A P b I_{3-x} C_{x}$ and $M A P b I_{3-x} B r_{x}$ are presented in Figure 2.3.

\section{Globularity factor}

Recent results have shown that the tolerance factor for more complex molecules than $M A$ and $F A$ needs to be reviewed. Kieslich et al. [109] first considered the nonspherical shape of the molecules to calculate improved tolerance factors. However, this model only applies to elemental and simpler organic cations. For more complex molecules, additional considerations such as rigidity, molecular asymmetry or acidity are needed. For this reason, Grätzel et al. [45], revised the cationic radius by proposing the molecular globularity, $g$, defined as

$$
g=\frac{S}{S_{e q}}
$$




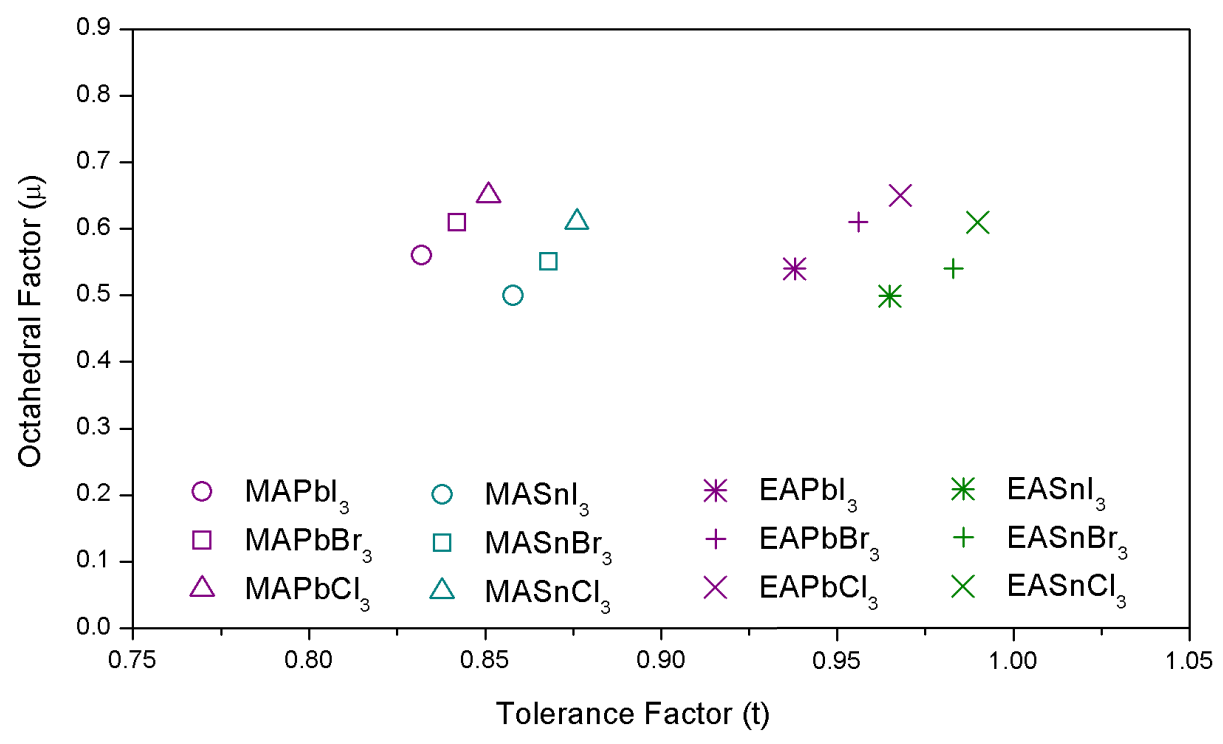

Figure 2.3: Comparison of aproximate $t$ and $\mu$ factors for 12 halide perovskites [108].

where $S$ is the actual molecular surface and $S_{e q}$ is the surface of a sphere of volume equal to the molecular volume. Molecular globularity increases from 1 as the molecular shape deviates from that of a sphere, accounting for molecular asymmetry. With the globularity factor, the tolerance factors (assuming a normalized tolerance factor of 1 for $F A$ ) of the cations ( $X A$, with $X=M, F, E, G, I)$ are defined by

$$
t=1-\left(\frac{r_{e q}(F A)}{g}-\frac{r_{e q}(X A)}{g}\right)
$$

where $r_{e q}$ is the radius of the sphere with the same volume as that calculated for the molecule. These calculations confirm the expected tolerance factor trend where $M A$ has a smaller tolerance factor than $F A$ and $E A$. $G A$ could still be in an accessible range, consistent with the report of partial $G A$ incorporation in perovskite films [110]. The main surprise, however, is $E A$, being even closer to the black perovskite formation regime than previously anticipated (more so 
even than $R b$ at the other end of the range). This renders $E A$ close to being compatible with $3 D$ perovskites and may be tolerated in large quantities.

\section{New Tolerance Factor}

One key aspect of the performance of $t$ is how well the sum of ionic radii estimates the interatomic bond distances for a given structure. Shannon's revised effective ionic radii, as a function of ion, oxidation state, and coordination number [111], is the most common data source for ionic radii. Most efforts to improve $t$ have focused on refining the input radii or increasing the dimensionality of the descriptor. However, up to now all applied approaches for improving the Goldschmidt tolerance factor have only been effective over a limited range of $A B X_{3}$ compositions.

The reason behind these attempts to improve $t$ is that its accuracy has been reported often insufficient. For example, considering $576 A B X_{3}$ solids experimentally characterized at ambient conditions, $t$ correctly distinguishes between perovskite and nonperovskite for only $74 \%$ of materials and performs worse for compounds containing heavier halides (chlorides - 51\% accuracy, bromides $-56 \%$, and iodides $-33 \%$ ) than for oxides $(83 \%)$ and fluorides $(83 \%)$.

Employing the SISSO (Sure Independence Screening and Sparsifying Operator) approach to identify an improved tolerance factor for predicting whether a given compound will be stable as perovskite, J. Bartel et al. [112] defined a new tolerance factor, $\tau$, as

$$
\tau=\frac{r_{X}}{r_{B}}-n_{A}\left(n_{A}-\frac{\frac{r_{A}}{r_{B}}}{\ln \left(\frac{r_{A}}{r_{B}}\right)}\right)
$$

where $n_{A}$ is the oxidation state of $A$ and $r_{i}$ is the ionic radius of ion $i$. This new tolerance factor $\tau$ allows to achieve a $92 \%$ overall accuracy and 
nearly uniform performance across the five anions evaluated (oxides - 92\% accuracy, fluorides $-92 \%$, chlorides $-90 \%$, bromides $-93 \%$, iodides $-91 \%$ ). The prediction of perovskite stability using $\tau$ requires only the chemical composition. The monotonic nature of $\tau$ allows for the determination of perovskite probability as a continuous function of the radii and oxidation states of $A, B$, and $X$. Using $\tau$, the probability of inorganic and organic-inorganic double perovskite formation for thousands of unexplored compounds has been predicted, resulting in a library of around 90,000 new stable perovskites for use in various applications, including photovoltaics. A repository containing all files necessary for classifying $A B X_{3}$ and $A A^{\prime} B B^{\prime}\left(X X^{\prime}\right)_{6}$ compositions as perovskite or nonperovskite using $\tau$ is available at [113].

\subsection{Components of Typical Perovskite Solar Cells}

This section provides a description of each component of perovskite-based solar cells, presenting also updated information on the research carried out on these components.

\subsubsection{The Compact Layer}

The compact layer, also known in the literature as blocking layer, is a pinhole-free compact, continuous $n$-type contact layer. The compact layer is intended to prevent, or slow down as much as possible, the recombination between the electrons injected into the conductive oxide layer and the holes in the perovskite. It is usually composed of the same material as the one used in the mesoporous layer, and it is uniformly deposited onto the conductive glass substrate. Blocking layers of $\mathrm{TiO}_{2}$ deposited by spray pyrolysis have shown to be compact, uniform and significantly thin. Other alternative methods to spray pyrolysis have been successfully employed, such as atomic layer 
deposition or spin-coating. Apart from $\mathrm{TiO}_{2}$, compact layers can use other metal oxides such as $\mathrm{TiCl}_{4}$ or $\mathrm{SnO}_{2}$, which have been reported to provide PCEs over $21 \%[114,115]$.

\subsubsection{The Mesoporous Layer}

The mesoporous layer consists of a thin mesoporous film of metal oxide nanoparticles deposited on top of the blocking layer. $\mathrm{TiO}_{2}$ is actually the most employed material for the mesoporous layer [29], due to its favorable properties, such as a wide bandgap of $3.2 \mathrm{eV}$ (implying it only has parasitic absorption in the UV region of the solar spectrum), its nontoxic nature and a low price.

$\mathrm{TiO}_{2}$ is a $n$-type material doped by oxygen vacancies, its doping density depending on its crystalline phase (anatase, rutile and brookite) and on its processing. The three phases have been studied in depth, the rutile phase being the thermodynamically most stable phase at room temperature, while brookite and anatase show a good kinetic stability below $600^{\circ} \mathrm{C}$. Actually, the anatase phase of $\mathrm{TiO}_{2}$ allows a higher performance on DSSCs and PSCs, although the reason of this behavior has not been completely understood. Perovskite solar cells using the $\mathrm{TiO}_{2}$ rutile phase have also been successfully reported [116].

The $\mathrm{TiO}_{2}$ mesoporous layer has been employed in a configuration similar to that of $D S S C s$, where the perovskite sensitizer was fully infiltrated in the gaps between the particles $[46,117]$. It is known that the $\mathrm{TiO}_{2}$ framework provides a high contact area for electron injection in DSSC and $s s D S S C$ solar cells, increasing the projected electrode area and the external quantum efficiency. However, the role of this mesoporous layers is actually being questioned, since the highest performance devices only contain a thin mesoporous layer of about 
$100 \mathrm{~nm}$.

In perovskite solar cells, the diffusion length of free carriers have been shown to be ranging between $100 \mathrm{~nm}$ and $1 \mu \mathrm{m}$ [118]. When the diffusion length is about $100 \mathrm{~nm}$, the mesoporous layer collects the absorbed electrons before they recombine. In this case, the collection efficiency is given by

$$
\eta_{C}=\frac{1}{1+\left(\frac{d}{L_{D}}\right)^{2}}
$$

where $d^{2}$ is the mean square displacement that electrons need to travel towards the $\mathrm{TiO}_{2}$ surface (usually around $20 \mathrm{~nm}$ for mesoporous layers) and $L_{D}$ is the electron diffusion length. If the diffusion length is greater than $1 \mu \mathrm{m}$, the mesoporous layer could act as a high surface area electrode that reduces the effective impedance for charge transfer, when compared to a flat electrode.

Alternatives to $\mathrm{TiO}_{2}$ include $\mathrm{ZnO}$ [119,120], $\mathrm{ZrO}_{2} \quad$ [121] and $\mathrm{Al}_{2} \mathrm{O}_{3}$, although, up to date, they have reached lower efficiencies than $\mathrm{TiO}_{2}$.

\subsubsection{The Perovskite Absorber}

The perovskite layer is the photo-active layer sensitive to the visible region of the spectrum. The role of this layer is to absorb photons and transform them in free charges. $\mathrm{MAPbI}_{3}$ and related cubic and tetragonal perovskites are direct bandgap semiconductors. The bandgap of pure $\mathrm{MAPbI}_{3}$ has been reported to be about $1.57 \mathrm{eV}$ with an optical absorption onset at $800 \mathrm{~nm}$, and a high absorption coefficient between $10 \times 10^{4}$ and $10 \times 10^{5} \mathrm{~cm}^{-1}$ throughout the visible spectrum and part of the infrared [122]. It has been reported that, in order to obtain high PCEs, it is necessary to obtain high diffusion lengths. Although the electronic effect of grain boundaries is not yet entirely clear, it is key in order to obtain larger grain size, high crystallinity and low 
defect concentration. Most organic-inorganic perovskites are deposited from solution and cannot withstand prolonged heating treatments. Since 2012, many studies have focused on compositional engineering and optimization of the deposition. Initially, $M A P b I_{3}$ was deposited by preparing concentrations of the precursor materials $\left(\mathrm{PbI}_{2}\right.$ and $\left.M A I\right)$ in a polar solvent, such as $\gamma$-butyrolactone $(G B L)$, N,N-dimethylformamide $(D M F)$, dimethylsulfoxide $(D M S O)$ or 1-methyl-2-pyrrolidinone $(N M P)$, which, upon spin-coating, resulted in crystallization $[117,123]$.

In the following section, a brief abstract of perovskite preparation processes and deposition protocols are reported.

\subsubsection{The Hole Transport Layer}

In a perovskite solar cell, the hole transport layer (HTM) is responsible for selectively transporting holes and blocking electrons. It must be able to match the current generation rate of the perovskite and, thus, have a sufficiently low resistance. Many materials, such as inorganic $p$-type semiconductors $\mathrm{NiO}, \mathrm{WO}_{3}$ and $\mathrm{MoO}_{3}$, have been successfully employed as hole transporting materials in perovskite solar cells. However, the most commonly HTMs are solution-processable $p$-type organic materials, either molecular or polymeric, such as 2,2',7,7'-tetrakis-N,N-di-paramethoxyphenylamine-9,9'-spirobifluorene (spiro-OMeTAD) [124]. Spiro-OMeTAD has been successfully employed in $D S S C s$ and it is actually used for high efficiency perovskite solar cells.

Spiro-OMeTAD is an amorphous, wide bandgap organic semiconductor with high solubility in organic solvents. It has a glass transition temperature of $121^{\circ} \mathrm{C}$ and a melting point at $246^{\circ} \mathrm{C}$ [125]. Hole mobilities ranging from $1 \times 10^{-5} \mathrm{~cm}^{2} V^{-1} s^{-1}$ to $1 \times 10^{-4} \mathrm{~cm}^{2} V^{-1} s^{-1}$ have been reported. The first oxidation potential of spiro-OMeTAD was measured at $-5.1 \mathrm{eV}$ by 
photoelectron spectroscopy and electrochemical techniques. The oxidation potential matches the valence band of most hybrid perovskite materials, and then, the hole transfer should be favored, while conduction band electrons are not energetic enough to reduce spiro-OMeTAD. Typically, spiro-OMeTAD is deposited by spin-coating from a concentrated solution in chlorobenzene or toluene. Curiously, bare spiro-OMeTAD leads to very poor device performance.

Additives are usually employed in the formulation and spin-coated together with the HTM. The most commonly used additives are 4-tertbutylamine $(4-t B P$ or $t B P)$ and LiTFSI. In addition, molecular dopants are sometimes used to enhance the conductivity of the HTM. The presence of those specific additives originate from the formulation used in solid state dye cells, although their role in PCS is still not clear. Snaith showed that LiTFSI has an effect on the hole mobility of spiro-OMeTAD [126]. It has also been demonstrated that LiTFSI actually acts as a $p$-dopant in the presence of oxygen, increasing the rate of photo-oxidation of the HTM [127-129]. Despite the fact that the role of LiTFSI remains not completely clear, avoiding the use of $4-t B P$ and LiTFSI has been difficult. Figure 2.4 shows the device architecture and cross-sectional FESEM image for a mesoporous oxide based perovskite solar cell using spiro-OMeTAD as the hole transport material.

\subsubsection{Back-contact}

Traditional thin film perovskite solar cells are back-contacted with a metallic layer, usually gold or silver. However, silver suffers from instability towards $\mathrm{MAPbI}_{3}$ as it oxidizes into $\mathrm{AgI}$. Semi-transparent contacts using silver nanowires $[130,131]$ and indium tin oxide counter electrodes sputtered on $M o O x$ have also been successfully used to assemble 2-terminal tandem 


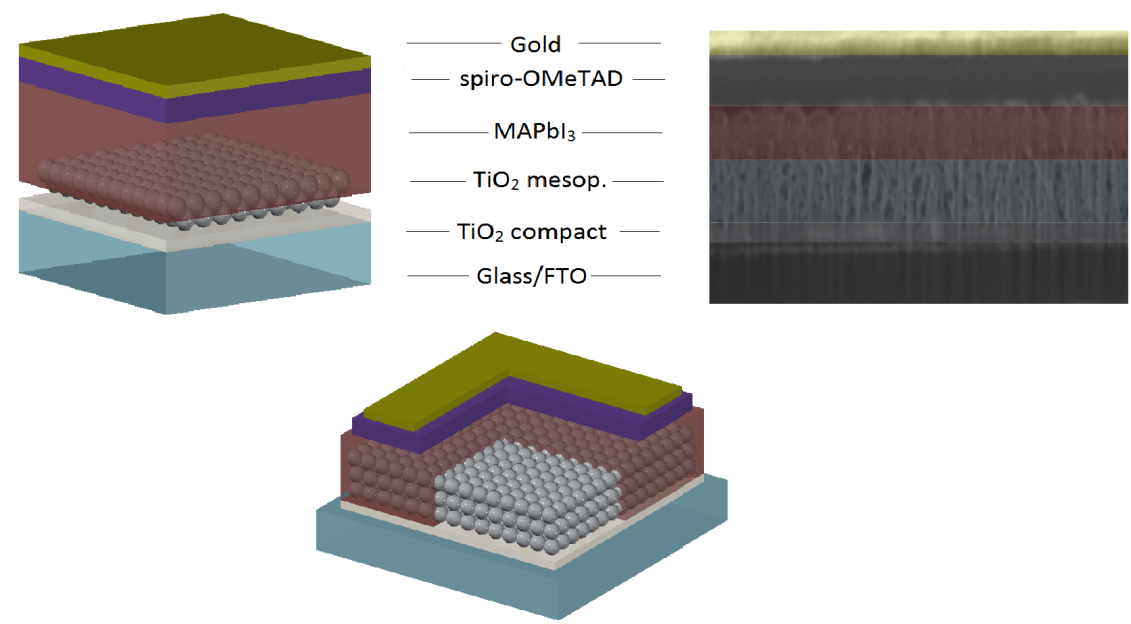

Figure 2.4: Device architecture and cross-sectional FESEM image for a mesoporous oxide based perovskite solar cell.

Si/M APbI 3 solar cells $[132,133]$.

\subsection{Chemical and Physical Techniques}

One advantage of organometal halide perovskites is their flexible processability. Perovskite films can be prepared by chemical and physical deposition techniques, mostly including one-step solution processing, two-step sequential deposition, vapor deposition and vapor assisted solution processing. It is worth noting that perovskite film quality is susceptible to deposition conditions. Therefore, it is important to gain further knowledge of processing methods, which can enable further improvements. In this section, some representative methods are described.

\subsubsection{Solution Processes}

Solution-process manufacturing methods for perovskites in PSCs can be divided into two categories, depending on the number of steps required 
to form the perovskite material. In the one-step methods, all perovskite precursors are dissolved in a single solution and deposited onto a substrate. Then, after the evaporation of the solvent, the perovskite material forms. These methods were the first manufacturing processes to be researched on solid-state PSCs $[49,51]$. The solvents, which are capable of dissolving the precursor materials, are typically high-boiling point solvents such as $\gamma$-butyrolactone (GBL), N,N-dimethylformamide $(D M F)$, dimethyl sulfoxide (DMSO), N-methyl-2-pyrrolidone $(N M P)$ and dimethyl-acetamide $(D M A$ or $D M A c)$ [134]. The advantage of one-step methods is the compositional control during the manufacturing processes. This method can be complemented by an extra step in which the perovskite film is annealed at a specific temperature to finalize the reaction, improve the crystal quality as well as film morphology [135].

On the other hand, two-step methods usually consist of a stepwise perovskite precursor addition, also known as sequential deposition, to form the perovskite. Organic-inorganic perovskite materials were first synthesized employing a two-step method by Liang et al. in 1998, and later in 2013, Burschka et al. successfully applied these methods to PSCs [136,137]. With these two-step methods, the number of parameters that can be controlled is increased. The main benefits of employing two-steps methods are an improved crystallization and the possibility of different deposition techniques.

Apart of these categories, other methods may need further processing steps in order to modify the perovskite material or the precursors formed sequentially. 


\subsubsection{Deposition Techniques}

\section{Spin-coating}

Spin-coating is the most employed technique to deposit perovskite thin films from solution with homogeneous thickness, given its reliability and simplicity of application. Almost all high-efficiency PSCs employ spin-coating in one (or more) steps of the device preparation.

Deposition by the spin-coating method involves the application of a solution onto a substrate, followed by spinning the substrate at a high rate, usually several thousand rounds-per-minute. Two physical principles play a fundamental role in the mechanics of spin-coating: the centrifugal force, arising from the rotating motion of the substrate, that causes the solution to be spread outwards from the center, and the surface tension of the solvent, that holds the solution together while the centrifugal force pulls it apart.

Depending on how the solution is applied on the substrate, spin-coating techniques can be split into two categories: static and dynamic. Static spin-coating involves the deposition of the solution while the substrate is not moving. When using a static dispense it is usual to coat the entire substrate (or at least all of the active part of the substrate) before starting the spin coating. Dynamic spin-coating then refers to a spinning substrate onto which the solution is dispersed, sometimes also called spincasting.

Despite its ability to produce thin films of highly uniform thickness, spin-coating is not good enough for upscaling of thin film manufacturing. During the application process most of the solution is flung aside and, unless specifically recycled, wasted. The rotating motion also results in a higher velocity of the substrate the further away it is from the center of rotation. This means that the solution will experience a larger force on the edges of the substrate than in its center, which can result in a non-uniform layer thickness 
for large substrates.

\section{Sequential Deposition in Two-steps}

For a sequential deposition procedure of the perovskite, the most commonly employed technique is to dip a solid $\mathrm{Pb}_{2}^{+}$powder precursor film into a chemical bath solution which contains the respective cations and anions. There are many chemical combinations available and many have already been studied [138]. The sequential deposition of $\mathrm{MAPbI}_{3}$ consists of a two-step process. In the first step, $\mathrm{PbI}_{2}$ is infiltrated into $\mathrm{TiO}_{2}$ nanopores, forming a thin coverage layer. In the second step, lead iodide is converted almost instantaneously into $\mathrm{MAPbI}_{3}$ by dipping the film into a $M A I$ solution in 2-propanol (IPA). $\mathrm{PbI}_{2}$ is insoluble, while $M A I$ dissolves easily in $I P A$. The $M A P b I_{3}$ perovskite will, however, dissolve in IPA but the time it takes is longer than the reaction time to form the perovskite film. With this method, large grains of high quality, better coverage and uniformity have been achieved. PCEs of about $15 \%$ were initially achieved, reaching later efficiencies of up to $17 \%$ [139]. Im et al. showed that the crystal size and growth rate of $M A P b I_{3}$ is strongly affected by the concentration of $M A I$ [139], which also affects the light harvesting efficiency and the hole collection rate.

\section{Vapor-assisted Deposition}

The vapor-assisted deposition method has been demonstrated as an effective way to deposit uniform and dense perovskite films. This deposition method involves partial or total deposition of the perovskite layer through a carrier gas. Leyden et al. used a multi-zone tube oven to deposit both $M A I$ and $\mathrm{PbI}_{2}$ from two different sources, under relatively low vacuum (100 $\mathrm{Pa}$ ) using a $N_{2}$ carrier gas and efficiencies of $11.8 \%$ [140]. Alternatively, Chen et 
al. have proved that vapor-assisted conversion $(V A S P)$ of $\mathrm{PbI}_{2}$ to $\mathrm{MAPbI}_{3}$ was achievable by placing $\mathrm{PbI}_{2}$-coated films in a closed petri dish containing $M A I$ powder. When the dish is heated to $150^{\circ} \mathrm{C}$, the $M A I$ vaporizes and converts lead iodide into perovskite with full surface coverage and grain size up to microscale. Efficiencies of $16.8 \%$ have been achieved employing this deposition $[141,142]$.

\section{Thermal Evaporation}

Mitzi et al. [143-145] employed thermal perovskite evaporation from its precursors $\mathrm{PbI}_{2}$ and $M A I$ to deposit $M A P b I_{3}$ as the photo-active layer in a solar cell $[146,147]$. There are different techniques for thermal evaporation. Typically, thermal evaporation takes place when placing $P b I_{2}$ and $M A I$ in two separate thermal evaporation boats and heated together under vacuum [148]. However, Teuscher et al. have shown that regulating the chamber pressure, with a thermal regulator on the $M A I$ source, affects the final $I / P b$ ratio in the device [149]. In addition, it has been shown that $\mathrm{PbCl}_{2}$ can also be used as a source of evaporation together with $M A I$, to form efficient solar cells [150]. Single source evaporation of perovskite single crystals has shown that the perovskite can be deposited with a high level of purity, while retaining the proper nominal $\mathrm{MAPbI}_{3}$ stoichiometry [151]. By flash evaporation of meniscus-coated perovskite films, Longo et al. demonstrated that single-source evaporation of the perovskite could compete with dual-source evaporation, and achieve $P C E$ of up to $12.2 \%$ [152].

\section{Vacuum flash-assisted deposition}

In this deposition method, after spin-coating a perovskite precursor containing $\mathrm{Pb}_{2}^{+}, \mathrm{Br}^{-}, \mathrm{I}^{-}, M A^{+}$and $F A^{+}$in a mixture of $D M S O, D M F$ and 
$G B L$, the substrate is placed in a vacuum oven at $20 \mathrm{~Pa}$ for $10 \mathrm{~s}$, followed by annealing at $100^{\circ} \mathrm{C}$ under atmospheric pressure for 30 minutes. Device fabrication then follows the traditional route of spiro-OMeTAD deposition and gold back-contact evaporation [153]. This deposition technique has achieved high PCE perovskite solar cells (up to 20.5\%), and smooth uniform films with large perovskite grains, without the need of any anti-solvent.

\subsubsection{Morphology Control}

\section{Thermal Annealing}

Thermal annealing is required in most of the deposition techniques to remove residual solvents or additives and to crystallize the perovskite. However, this process affects the perovskite film formation inducing chemical and structural changes. Annealing temperature is a key parameter that favors the proper formation of the perovskite film. An annealing temperature between $80-100^{\circ} \mathrm{C}$ has been proved to be optimal for the film morphology. Snaith et al. found that rapid annealing at $130^{\circ} \mathrm{C}$ induced the growth of micron-sized perovskite crystal domains, while long yet moderate annealing at $100^{\circ} \mathrm{C}$ resulted in polycrystalline domains with a size of 100-1000 $\mathrm{nm}$ [154]. In addition to the annealing temperature, the thermal annealing time is also of importance since it has a significant impact on the film morphology of perovskites [155]. Thermal annealing at $105^{\circ} \mathrm{C}$ for $15 \mathrm{~min}$ has been proved to be efficient enough to conduct the formation of phase-pure perovskites. Additionally, if the thermal annealing time is extended by up to $2 h$, the crystallization and grain size of perovskites is increased, keeping full coverage uniformity and, therefore, leading to remarkably enhanced charge mobility and significantly increased fill factor $(F F)$ and short-circuit current density $\left(J_{S C}\right)$. However, a longer annealing time for $3 h$ decomposes perovskite. A PCE of 
$15 \%$ was reached for a planar perovskite solar cell fabricated employing the optimal annealing process. Stepwise annealing ramps are also a key factor in the formation of the perovskite films. They have been related with the solvent evaporation rate, which is particularly important for the nucleation and growth of the crystals [156].

\section{Solvent Annealing}

Solvent annealing is applied to improve the grain size and the crystallinity of perovskites, complementing the thermal-annealing process. It consists of the introduction of solvent vapor during perovskite crystallization. $D M F$ vapor annealing solubilizes the solid film, so that the precursor ions or molecules are able to diffuse a longer distance than when employing thermal annealing. A solvent-annealed $\mathrm{MAPbI}_{3}$ film with an average grain size of up to $1 \mu \mathrm{m}$, larger than the maximum grain size in thermally annealed films (around $260 \mathrm{~nm}$ ), has been achieved [157]. Additionally, solvent-annealed films exhibited other key properties capable of improving device performance such as, reduced trap density, improved charge recombination lifetime, lower charge extraction time, and better carrier diffusion length. Besides $D M F$, vapor annealing under $D M S O$ or mixed $D M F /$ chlorobenzene solvents have been demonstrated to improve perovskite crystallization $[158,159]$. The combination of solvent annealing and thermal annealing have provided a high-quality perovskite [160] exhibiting high uniformity, large grain size up to $1.1 \mu \mathrm{m}$ and a PCE of $14 \%$.

\section{Anti-solvent Crystallization of the Perovskite Film}

Among the many different methods introduced to improve the surface morphology of a perovskite film, gas-blowing [161], amino halide addition [162-164], anti-solvent treatment (A.S.T.) [165,166], solvent vapor annealing 
$[167,168]$, and vacuum treatment [169], have been the most broadly employed post-treatment methods.

In 2014, Jeon et al. reported the first anti-solvent preparation technique for perovskite solar cells [170] applying a perovskite precursor solution with a mixture of $G B L$ and $D M S O$ solvents onto a mesoporous $\mathrm{TiO}_{2}$ substrate for spin-coating. During the spin-coating process, anti-solvent was injected onto the spinning substrate causing the $G B L: D M S O$ solvent to be expelled from the perovskite precursor film. The result was a highly even precursor film which, upon annealing, formed a highly crystalline and uniform perovskite film [170-173]. It is worth noting that with a A.S.T. several world record efficiencies have been reported [166, 174-178].

The anti-solvent technique is only used for one-step methods since all the required chemical components of the perovskite material must be present in the same solution. This technique involves the addition of a specific solvent, in which the perovskite is poorly soluble, to the precursor solution causing the perovskite to start crystallizing. Normally, the perovskite precursors are dissolved in high boiling point polar aprotic solvents such as $\gamma$-butyrolactone $(G B L)$, dimethylformamide $(D M F)$, dimethyl sulfoxide (DMSO), N-methyl-2-pyrrolidone $(N M P)$ and dimethylacetamide $(D M A c)$.

The working mechanism of an anti-solvent is to speed up heterogeneous nucleation through the creation of an instantaneous local supersaturation on the spinning substrate. During the anti-solvent treatment, and under the influence of several physicochemical properties of both solvents, complicated interactions are taking place simultaneously.

Thus far, the most popular anti-solvents for perovskite film formation are toluene $(\mathrm{Tol})$ [174], chlorobenzene $(C B)$ [176] and diethyl ether (ether) [178]. Although these three solvents perform efficiently with different 
perovskites they show completely different physicochemical properties. Other anti-solvents that have recently been studied are trifluorotoluene (TFT), p-xylene $(X y l)$, and dichloromethane $(D C M)$, deposited from a mixed $D M S O$ and DMF solution (1:4) [179].

Anti-solvents have shown to have a significant effect on the perovskite crystal growth kinetics and film morphology on a micrometer scale, due to differences in boiling point, miscibility, and dielectric constants. Anti-solvents with a low boiling point as well as poor miscibility with solution solvents will lead to lower efficiency and reproducibility compared to the anti-solvents with a higher boiling point and good miscibility with $D M F / D M S O$. Indeed, it has been shown that using a high boiling point and miscible anti-solvents, compact perovskite films with full coverage on the substrate, and PCEs above $18 \%$ are obtained. When using TFT to replace Tol and CB, a PCE of $20.3 \%$ has been reached.

It has been proved that a suitable A.S.T. serves not only to speed up heterogeneous nucleation but also to influence the initial crystal growth, leading to a pinhole-free homogeneous film morphology during thermal annealing [179].

\section{Atmospheric Effects}

Organometal halide perovskites are vulnerable to the environment and, therefore, controlling the atmosphere can help its crystallization. Thermal annealing in $\mathrm{O}_{2}$ has been found to increase $P C E$. This improvement may be due to the fact that $\mathrm{O}_{2}$ diffusion can help reduce defect density at the grain boundaries and within the bulk of perovskites $[180,181]$. In addition, when employing spiro-OMeTAD as the hole transporting layer, $\mathrm{O}_{2}$ treatment would cause $p$-type doping and, therefore, an increase in the electrical conductivity. 
Moisture-assisted crystal growth has also been demonstrated as an effective way to improve the film quality, grain size, carrier mobility, and lifetime of perovskites [125]. Due to the high hydrophilicity of $M A I$, an exposure to moderate moisture of a relative humidity $(R H)$ between $30 \%$ and $40 \%$ would cause the perovskite grain boundary to drag and merge adjacent grains, generating larger crystal grains.

Zou et al. have reported that spin-coating under low $R H$, accompanied by thermal annealing under high $R H$, improved perovskite crystallinity and device performance [126]. The reason for the improved crystallinity is that, at the spin-coating stage, low $R H$ leads to the formation of high nucleation density, leading to a high coverage of perovskite films, and, at the thermal annealing stage, the modest supersaturation induced by high $R H$ benefited the formation of perovskite films. Recently, it has been found that hydrated perovskite crystalline phases formed when exposed to water vapor at room temperature [127]. Also, both oxygen and moisture were found to greatly affect the luminescent properties of perovskites [128]. It has been demonstrated that perovskite films prepared by ambient annealing exhibited a comparable solar cell performance to that annealed in dry $N_{2}[129,182]$. The formation of $\mathrm{MAPbCl}_{3}$ phase was inhibited when annealing in air with $\mathrm{RH}$ of $50 \%$, which favored $\mathrm{MAPbI}_{3}$ crystallization. As a result, thermal annealing in air led to a significant increase of both crystallinity and crystal size of $\mathrm{MAPbI}_{3}$ as compared to that in nitrogen [183].

\subsection{The Physics of Solar Cells}

\subsubsection{Introduction}

Electromagnetic radiation consists of electric and magnetic disturbances that propagate as waves. The two components of an electromagnetic wave 
are mutually perpendicular and perpendicular to the direction of propagation. The electric field acts on both stationary and moving charged particles, but the magnetic field acts only on moving charged particles. Electromagnetic waves travel through vacuum at a constant speed called the speed of light, $c$, which has a value of $c=2.997 \times 10^{8} \mathrm{~m} / \mathrm{s}$.

A wave is characterized by its wavelength, $\lambda$, defined as the distance between two consecutive peaks of the wave. Electromagnetic radiation visible to the human eye (light) has a wavelength between $420 \mathrm{~nm}$ (violet light) and $700 \mathrm{~nm}$ (red light). The properties of a wave can be expressed in terms of its frequency, $\nu$, defined by the number of oscillations in a time interval divided by the duration of the interval. Frequency is given in Hertz, $H z$, with $1 \mathrm{~Hz}=1 \mathrm{~s}^{-1}$. Light has a frequency range between $710 \mathrm{THz}$ (violet light) and $430 \mathrm{THz}$ (red light). The period of an oscillation is the reciprocal of the frequency, i.e., $\frac{1}{\nu}$. During this period the wave propagates by one complete wavelength $\lambda$. The speed of propagation can be written as the quotient between the distance of propagation during one period of oscillation and the period of one oscillation, i.e.,

$$
c=\lambda \nu
$$

Classical mechanics, which has its origins in the laws of motion of Isaac Newton in the 17th century, can predict a precise trajectory for particles, with specific locations and momenta at each instant. Nevertheless, classical mechanics fails when applied to the transfer of very small energies and to objects of very small mass. In the late 19th century and in the early 20th century, three experiments led to the formulation of the quantum theory. One proved that energy was transferred only in discrete amounts, the second stated that electromagnetic radiation also behaves like a stream of particles, and the 
third showed that electrons, supposed to be particles, also behave like waves. Therefore, all electromagnetic radiation, including sunlight, can be considered as composed of particles, called photons, carrying specific amounts of energy that depend on the spectral properties of the source. The equation relating these two points of view is

$$
E_{\lambda}=\frac{h c}{\lambda}
$$

where $E_{\lambda}$ is the photon energy, $\lambda$ is the wavelength, $h$ is the Planck's constant and $c$ is the speed of light. The intensity of a light beam is defined as the number of photons it contains. Since the energy of the photons depends on the wavelength, the spectral composition of sunlight has to be taken into account when designing a solar cell.

\subsubsection{Air Mass}

The surface temperature of the Sun is about $5762 K$ and its radiation spectrum can be approximated by a black body radiator, whose emission is uniform in all directions. Only the photons emitted directly to the Earth contribute to the solar spectrum. Since the Earth is about 150 million kilometers away from the Sun, the light reaching the Earth can be considered parallel streams of photons. The radiation intensity above the Earth's atmosphere, or solar constant, is about $1.353 \mathrm{~kW} / \mathrm{m}^{2}$ and the spectral distribution is called air mass zero $(A M 0)$ radiation spectrum [184]. Air mass is defined by

$$
\text { Airmass }=\frac{1}{\cos \theta}
$$

where $\theta$ is the angle of incidence ( $\theta=0$ when the Sun is directly in the zenith) and it is used to measure how absorption in the atmosphere affects the spectral content and intensity of the solar radiation. From the definition, since $\cos \theta$ 


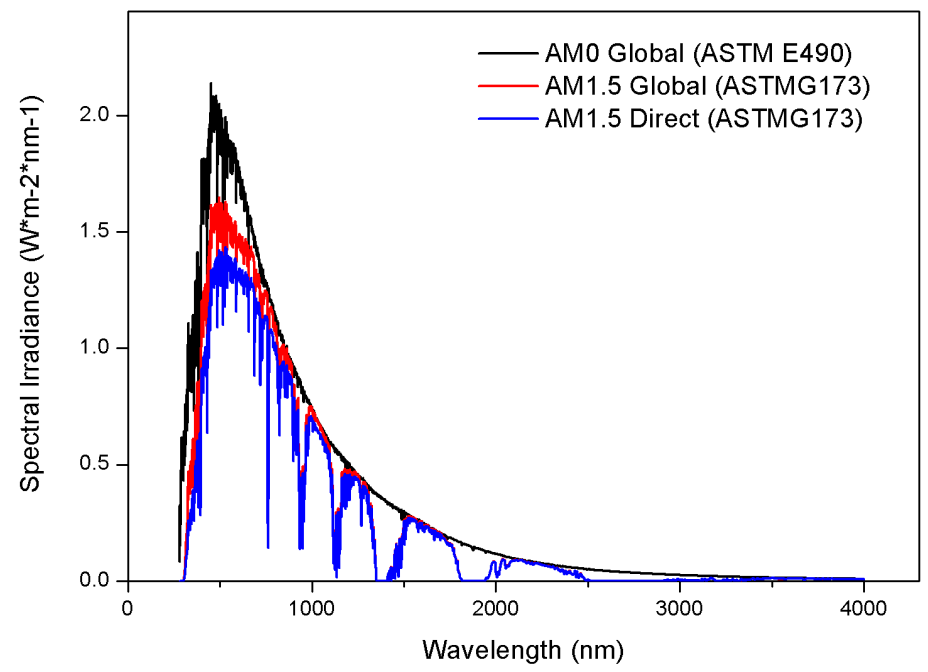

Figure 2.5: $A M 0$ spectrum compared with $A M 1.5$ spectrum (global and direct).

is positive and less than or equal to one for $\theta$ between $-90^{\circ}$ and $90^{\circ}$, the air mass number is always equal to one or greater.

Air mass number 1.5 spectrum, $A M 1.5$, normalized to a total power density of $1 \mathrm{~kW} / \mathrm{m}^{2}$, is reached when $\theta=48.2^{\circ}$, and it is normally used for comparing solar cell performances. On the Earth's surface, sunlight spectral content has a diffuse (indirect) component, mainly due to scattering and reflection in the atmosphere. This diffuse component can be up to $20 \%$ of the light incident on a solar cell. $A M 1.5 \mathrm{~g}$ (global) spectrum is defined including the diffuse component, in opposition to $A M 1.5 d$ (direct) that does not include it [185]. $A M 0$, and $A M 1.5 \mathrm{~g}$ (global and direct) are depicted in Figure 2.5.

\subsubsection{Properties of Photovoltaics}

Photovoltaics $(P V)$ transform solar radiation to usable energy by pushing electrons from a low-energy state to a higher energy state. $P V$ devices are 


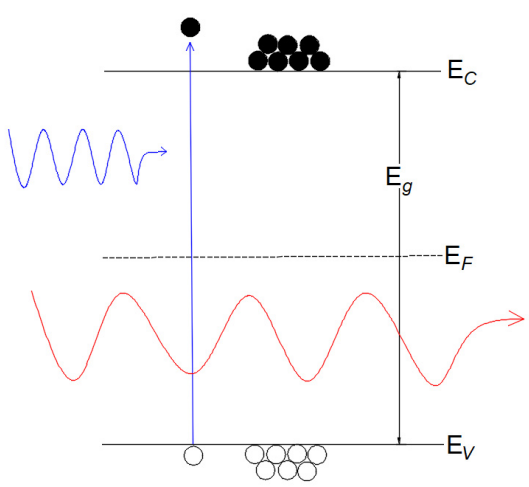

Figure 2.6: Schematic band diagram of a semiconductor. Photons with energy greater than $E_{g}$ (in blue) can be absorbed, creating an electron-hole pair.

made from semiconductors, which are reviewed in this section. The bandgap $\left(E_{g}\right)$ is one of the key elements necessary to understand the mechanism of a $P V$ device. The bandgap consists of disallowed energy levels introduced by the periodic potential of atoms in a solid [186,187]. The bandgap is defined as the energy difference between the upper edge of the valence band $\left(E_{V}\right)$ and the lower edge of the conduction band $\left(E_{C}\right)$. The chemical potential of electrons, known as the Fermi level $\left(E_{F}\right)$, is the maximum energy level that would be filled at $0 K$. At finite temperatures, the Fermi level defines the energy level at which the occupancy of that state is $50 \%$. In a semiconductor, the Fermi level lies within the bandgap, however thermal excitation at finite temperatures means that there is a population of electrons in the conduction band at equilibrium. By using a single semiconductor with, for example, an $E_{g}$ of $1.5 \mathrm{eV}$, all photons of higher energy will generate charge-carriers with an energy corresponding to the $E_{g}$ and photons with lower energy than the $E_{g}$ will not be absorbed. A schematic band diagram is shown in Figure 2.6 [187].

The ideal $E_{g}$ of a photoabsorber for use in a solar cell depends on the 


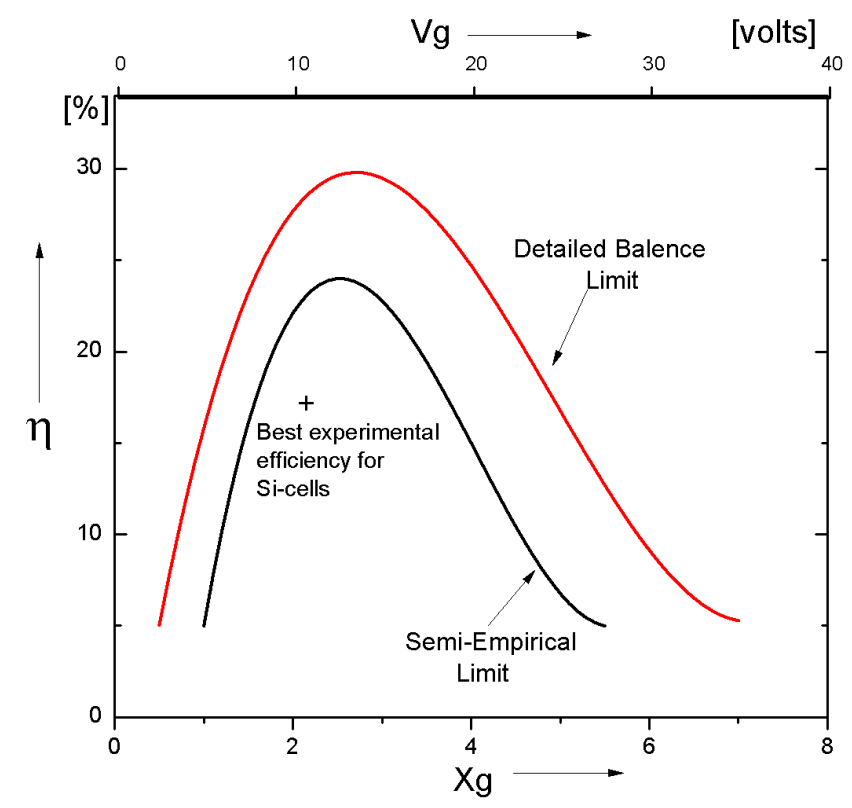

Figure 2.7: Figure based on the original work by Shockley and Quisser showing the theoretical maximum achievable $\eta$ for single-junction solar cells as a function of the bandgap, denoted here $V_{g}$ for fidelity to the original work $[188,190]$.

light emission spectrum of the radiating body. In 1961, Shockley and Queisser published a detailed balance $P C E$ limit of single-junction semiconductor solar cells, known as the Shockley-Queisser $(S Q)$ limit, which is depicted in Figure 2.7 [188]. As can be seen, the highest achievable $P C E$ for a single-junction solar cell is around 30\% although, recent research has established this limit at $33.8 \%[189]$.

\section{Semiconductors}

Semiconductor materials are used in solar cells due to their capability to absorb light and deliver a portion of the absorbed photon energy to carriers 
of electrical current (electrons and holes). A semiconductor diode is formed when an $n$-type semiconductor and a $p$-type semiconductor are put in contact to form a junction. In this section, basic physical principles underlying the operation of solar cells are studied. Definition of the basic solar cell figures of merit, like the open-circuit voltage $\left(V_{O C}\right)$, short-circuit current $\left(I_{S C}\right)$, fill factor $(F F)$, conversion efficiency $(\eta)$ and the collection efficiency $\left(\eta_{C}\right)$ are also introduced.

\section{Fundamental Properties}

The electronic structure of a solid accounts for electrical, optical and magnetic properties, that can have technological applications. Some solids, particularly metals, conduct electricity as they have mobile electrons. These electronic conductors are classified on the basis of the variation of their electrical conductivity with temperature. Therefore, a metallic conductor is an electronic conductor with a conductivity that decreases as the temperature is raised. Metallic conductors include metallic elements, their alloys and graphite. A semiconductor is an electronic conductor with a conductivity that increases as the temperature is raised [191], and includes, among others, silicon and gallium arsenide. Generally, a semiconductor has a lower conductivity than that typical of metals, but the magnitude of the conductivity is not relevant to the distinction. Conventionally, substances with very low electrical conductivities are classified as insulators. Superconductors are substances that conduct electricity with zero resistance [191].

To understand the origins of electronic conductivity in conductors and semiconductors, it is necessary to study the consequences of the formation of bands in different materials. At $T=0$, only the lowest molecular orbitals are occupied, up to the Fermi level. The availability of empty orbitals just 
above the Fermi level accounts for the mobility of the electrons and hence the electrical conductivity. At temperatures above zero, atoms vibrate, and collisions between the moving electrons and atoms are more likely. This means that the electrons $\left(e^{-}\right)$are scattered out of their paths through the solid and are less efficient charge transporters. As the temperature is increased, electrons leave the lower band, called valence band and populate the empty orbitals of the upper band, called conduction band. As a consequence of the electron movement, positively charged holes are left in the valence band. A hole $\left(h^{+}\right)$ can be described as a positively charged quasi-particle defined by the absence of an electron. These holes and the promoted electrons are now mobile, and the material is now a semiconductor, because the electrical conductivity depends on the number of electrons that are promoted across the gap, and that number increases as the temperature is raised. If the gap between the valence band and conduction band is wide, very few electrons can be promoted at room temperature $(R T)$ and the conductivity remains close to zero, resulting in an insulator.

A method to increase the number of charge carriers (electrons and holes) and enhancing the semiconductivity of a solid is to add foreign atoms, called dopants, into a pure semiconductor. These dopants can trap electrons, withdrawing electrons from the filled band and leaving holes, allowing also the remaining electrons to move. The doping procedure gives rise to $p$-type semiconductors, the $p$ indicating that the holes are positive relative to the electrons in the band. Alternatively, a dopant can carry excess electrons, and these additional electrons occupy otherwise empty bands, originating $n$-type conductivity, the $n$ denoting the negative charge of the carriers.

A solar cell usually consists of a photoabsorber, which absorbs light to excite electrons $\left(e^{-}\right)$, and two selective contacts to spatially separate the 
photogenerated charges, i.e. the excited $e^{-}$and the hole $h^{+}$. Without any physical separation of the photogenerated charges to the two different electrodes no photovoltage will appear. This separation of charges can be introduced, for example, by creating an electric field in the material of the solar cell, which will cause the $e^{-}$to move in one direction and $h^{+}$in the other. The charge separation results in a current flow and, along with the potential difference between the electrodes, generates electric power when connected to an external circuit. Charge separation by an electric field is common in solar cells which are based on a $p$ - $n$ junction, such as crystalline silicon $(c-S i)$ solar cells. Solar cell materials are chosen on the basis of their absorption characteristics and their production cost. They can be made from different semiconductor materials. Crystalline, polycrystalline and amorphous silicon $(S i)$ are the most commonly employed materials to manufacture solar cells, due to the fact that their absorption characteristics match approximately the solar spectrum. Other semiconductor materials, such as GaAs, GaInP, $\mathrm{Cu}(\mathrm{InGa}) \mathrm{Se}_{2}$ or $\mathrm{CdTe}$ have also been used to manufacture solar cells.

\section{The $p$ - $n$ Junction}

The simplest $P V$ device is a $p-n$ homojunction diode. A $p-n$ junction is formed when semiconductor slabs with different concentrations of charge carriers, electrons and holes, are brought into electrical contact. A homojunction means that the material employed is the same, other than the doping level, on both sides of the junction.

In a pure semiconductor the Fermi level would be in the middle of the bandgap, but impurities in the material, called dopants, lead to a higher concentration of one of the two carrier types. An electron-rich slab is doped with electron donors and is said to be $n$-type, while a hole-rich slab is doped 
with electron acceptors and is said to be $p$-type.

The law of mass action states that the product of the electron and hole densities is constant at equilibrium, therefore the hole concentration in an $n$-type material and the electron concentration in a $p$-type material are reduced. The less abundant carrier in a semiconductor is called the minority carrier, and we shall see that solar cells depend on collection of these minority carriers.

When the $p$ - and $n$ - type slabs are connected, diffusion of electrons and holes occurs until equilibrium is reached, so that the Fermi level $\left(E_{F}\right)$ is constant across the diode. However, in the process of reaching equilibrium the electrons and holes leave behind fixed charges in the vicinity of the junction, with positive charge left behind on the $n$-type side and negative charge left behind on the $p$-type side.

This region is known as the depletion region (because the concentration of free charges is low) or the space charge region (because of the fixed charges present in this region). These fixed charges of opposite polarity produce an electric field and, therefore, a potential difference, called the built-in potential $\left(V_{b i}\right)$, between the two semiconductor slabs. Figure 2.8 shows a schematic $p$ $n$ junction.

The magnitude of this built-in potential is such that, at equilibrium, there is no net current across the diode, as the rate of diffusion across the depletion region is cancelled out by the rate of thermally activated charges being swept by the electric field across the junction. However, if a positive external bias is applied $(V>0)$, this reduces the magnitude of the electric field. Now, the electrons on the $n$-type side have a smaller energetic barrier to reach the $p$ type side. 
a)

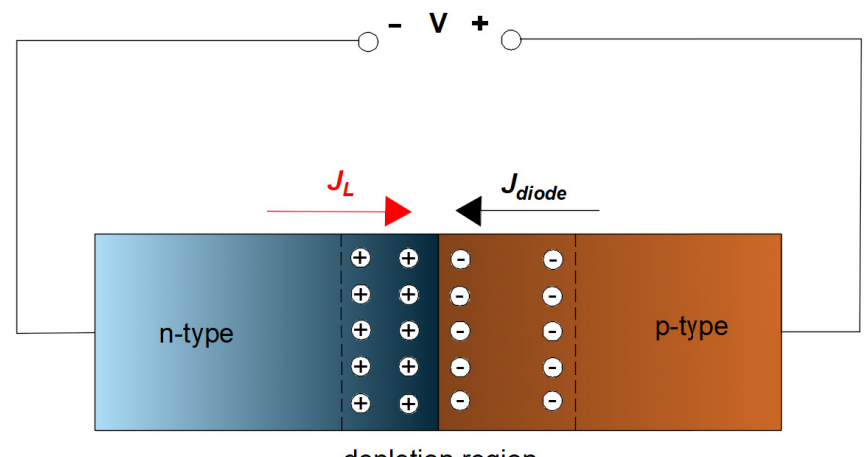

b)

depletion region

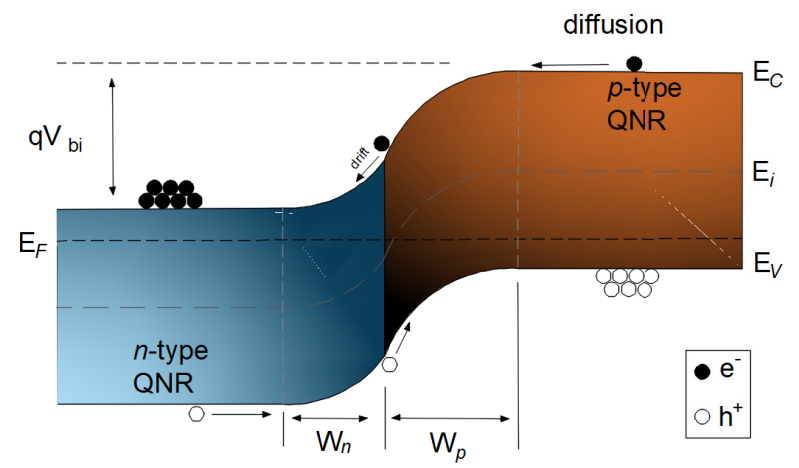

Figure 2.8: (a) Schematic plot of a $p$-n junction solar cell. (b) Energy band level diagram of a $p-n$ solar junction.

As the energetic distribution of electrons at thermal equilibrium has an exponential tail, an incremental decrease in the potential barrier produces an exponential increase in diffusive current across the diode. However, if a negative bias is applied $(V<0)$, the magnitude of the electric field is enhanced, but because the minority carrier population is small, the increase in electric field-driven drift current is low. Therefore, the built-in field provides rectification - it is easier to drive current in the forward direction than in the reverse direction. The properties of the $p$ - $n$ junction that we have developed qualitatively are described quantitatively by the ideal diode equation, also 
called the Shockley equation

$$
J(V)=J_{0}\left(e^{\frac{q V}{n k T}}-1\right)
$$

where $J$ is the current density, $q$ is the elementary charge, $n$ is the diode ideality factor, $k$ is Boltzmann's constant, $T$ is temperature, and $J_{0}$ is the reverse saturation current density, also called the leakage current density. Furthermore, bias modulates the width of the depletion region. The width of the depletion on the $n$-type side of the junction $\left(w_{n}\right)$ is given by [192]

$$
w_{n}(V)=\sqrt{\frac{2 \varepsilon_{r} \varepsilon_{0} n_{A}\left(V_{b i}-V\right)}{q n_{A}\left(n_{A}-n_{D}\right)}}
$$

where $\varepsilon_{r}$ is the relative static permittivity, $\varepsilon_{0}$ is the vacuum permittivity, $n_{A}$ is the acceptor density on the $p$-type side, and $n_{D}$ is the donor density on the $n$-type side.

So far, only a $p-n$ junction is in equilibrium in the dark has been discussed. However, when a semiconductor is illuminated, so that some photons have energy greater than the bandgap, optical absorption will generate additional electrons and holes on top of the thermally activated population. Once the semiconductor has successfully absorbed a photon, an $e^{-}$is excited from the valence band to the conduction band, leaving behind an $h^{+}$in the $V B$ and resulting in the formation of an electron-hole pair $\left(e^{-}-h^{+}\right)$, also known as an exciton. Now, the minority carrier population is much higher, but minority carriers must diffuse across the quasi-neutral region $(Q N R$, the part of the slab that does not experience an electric field) to reach the depletion region. Once a minority carrier reaches the depletion region it is quickly swept across by drift transport.

In this process the photo-generated carrier has moved to a higher energy 
state, which is the photovoltaic effect. This new current source is called the photocurrent and it is negative in sign because it opposes the diffusive current. Therefore, the current density-voltage characteristics of the illuminated $p-n$ diode become

$$
J(V)=J_{0}\left(e^{\frac{q V}{n k T}}-1\right)-J_{L}(V)
$$

where $J_{L}$ is the photocurrent density. The bias-dependence of the photocurrent density is often overlooked. For $c$-Si solar cells, the photocurrent density is not modulated when bias is applied, since the depletion width is very narrow relative to the total thickness of the solar cell. In order to collect photo-excited carriers, $\mathrm{PbS}$-based quantum dot photovoltaic devices depend on drift transport from the built-in electric field $[187,193]$, therefore the photocurrent density decreases as the electric field diminishes under forward bias and the depletion region gets narrower (see Equation 2.18). Equation 2.19 provides a quantitative model for the current-density voltage characteristics in a diode.

The Shockley equation 2.17 applies to an ideal diode, but in reality parasitic resistances may reduce the power output of a solar cell and alter the current voltage characteristics so that the generalized Shockley equation is $[187,194]$

$$
J(V)=J_{0}\left(e^{\frac{q\left(V-J r_{s}\right)}{n k T}}-1\right)+\frac{V-J r_{S}}{A r_{P}}
$$

where $A$ is the device area, $r_{S}=A R_{S}$ and $r_{P}=A R_{P}$ are the specific series resistance and the specific parallel resistance, respectively. However, for the rest of this thesis we will only discuss results in terms of the ideal Shockley equation. In the next section the limits on power conversion efficiency for solar cells will be discussed. 


\section{Figures of Merit}

Short-Circuit Current The short-circuit current $I_{S C}$ is the current through the solar cell when the voltage across the solar cell is zero, i.e., when the solar cell is short-circuited. $I_{S C}$ is due to the generation and collection of light-generated carriers. For an ideal solar cell at most moderate resistive loss mechanisms, the short-circuit current and the light-generated current are identical. Therefore, the short-circuit current is the strongest current which may be drawn from the solar cell. $I_{S C}$ depends on many factors such as, the area of the solar cell, the number of photons, the spectrum of the incident light, the optical properties (absorption and reflection) of the solar cell and the collection probability of the solar cell. When comparing solar cells of the same material type, the most critical material parameter is the diffusion length and surface passivation. In a cell with perfectly passivated surface and uniform generation, the equation for the short-circuit current can be approximated as

$$
I_{S C}=q G\left(L_{n}-L_{p}\right)
$$

where $G$ is the generation rate, and $L_{n}$ and $L_{p}$ are the electron and hole diffusion lengths, respectively. Although this equation is only an approximation and does not fit the real behavior for the conditions encountered in most solar cells, the expression highlights that the short-circuit current depends strongly on the generation rate and the diffusion length. Silicon solar cells under an $A M 1.5$ spectrum have a maximum possible current of 46 $\mathrm{mA} / \mathrm{cm}^{2}$.

Open-Circuit Voltage The open-circuit voltage, $V_{O C}$, is the maximum voltage that a solar cell can deliver, when no current flows through the external circuit. Figure 2.9 shows $I-V$ curve together with the representation of $V_{O C}$. The open-circuit voltage corresponds to the amount 
of forward bias on the solar cell due to the bias of the solar cell junction with the light-generated current. $V_{O C}$ can be expressed as

$$
V_{O C}=\frac{n k T}{q} \ln \left(\frac{I_{L}}{I_{0}}+1\right)
$$

Equation 2.22 measures how open-circuit voltage depends on the saturation current of the solar cell and the light-generated current. The saturation current, $I_{0}$ depends on recombination in the solar cell, therefore $V_{O C}$ is a measure of the amount of recombination.

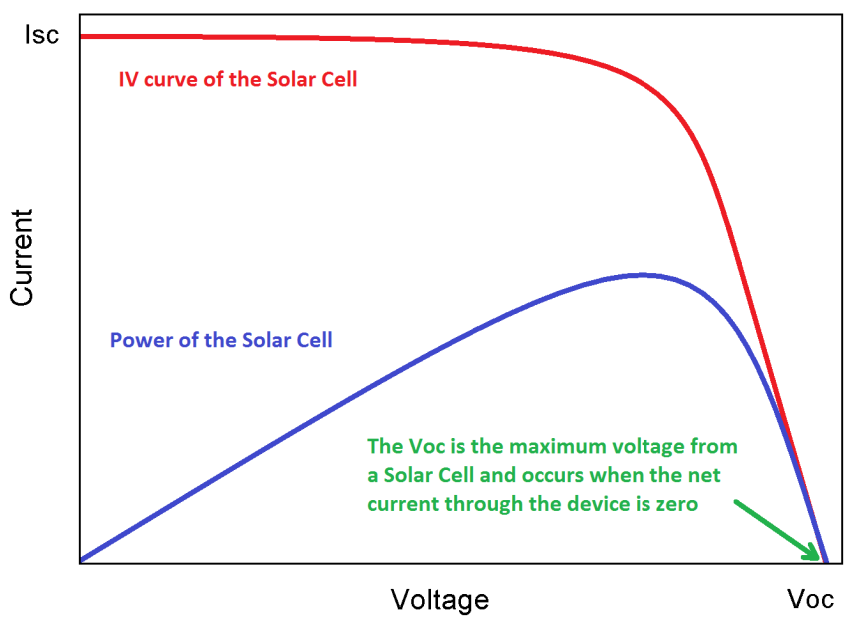

Figure 2.9: $I-V$ curve representation with $V_{O C}$, the maximum voltage delivered when no current flows through the external circuit.

$V_{O C}$ can be also expressed in terms of the bandgap $E_{g}$. It is well known that the short-circuit current decreases when the bandgap is increased, however the open-circuit voltage increases as the bandgap is increased. In an ideal device the $V_{O C}$ is limited by radiative recombination and the analysis uses the principle of detailed balance to determine the minimum possible value of the diode saturation current, $J_{0}$, in terms of $q$ (electronic charge), $\sigma$ (Stefan-Boltzman constant), $k$ (Boltmann constant), $T$ (temperature) and 
$E_{g}$. Employing the expression of $J_{0}, V_{O C}$ can be described as a function of $E_{g}$ so long as the voltage is less than the bandgap, as is the case under one sun illumination.

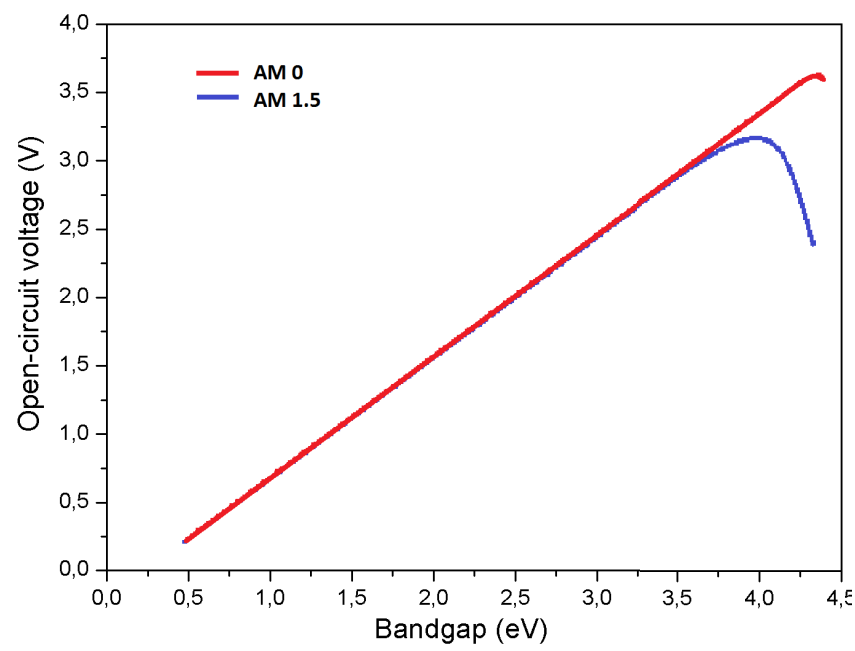

Figure 2.10: $V_{O C}$ as function of bandgap for a cell with $A M 0$ and $A M 1.5$. $V_{O C}$ increases with bandgap as the recombination current falls. There is drop off in $V_{O C}$ at high bandgaps due to the low $I_{S C}$.

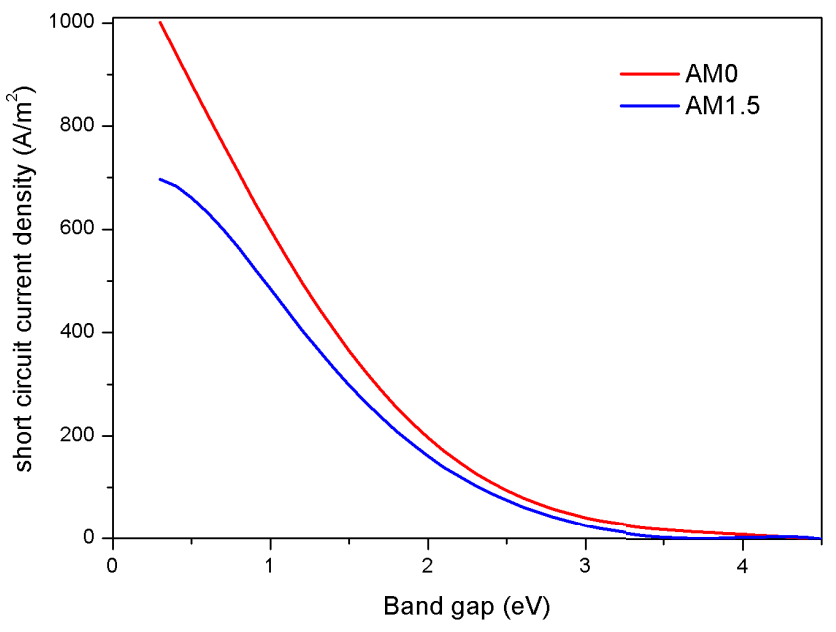

Figure 2.11: Short-circuit current density as a function of the bandgap under $A M 0$ and $A M 1.5$ spectrum. 
Fill Factor The fill factor $(F F)$ is defined as the ratio of the maximum power from the solar cell to the product of $V_{O C}$ and $I_{S C}$. The $F F$ can be seen as the area of the largest rectangle which will fit in the $I$ - $V$ curve and measures the squareness of the solar cell (Figure 2.12).

Assuming that the solar cell behaves as an ideal diode, the fill factor can be expressed as a function of $V_{O C}$,

$$
F F=\frac{v_{O C}-\ln \left(v_{O C}+0.72\right)}{v_{O C}+1}
$$

where

$$
v_{O C}=\frac{q V_{O C}}{k T}
$$

is a normalized voltage. Equation 2.23 is a good approximation of the ideal value of $F F$ for values of $v_{O C}$ greater than 10 . The $F F$ does not change drastically with a change in $V_{O C}$.

In practice, the $F F$ will be lower due to the presence of parasitic resistive losses. Thus, the FF is most commonly determined from measurement of the $I-V$ curve and is defined as

$$
F F=\frac{J_{M P P} V_{M P P}}{J_{S C} V_{O C}}
$$

where $J_{M P P}$ and $V_{M P P}$ are the current density and voltage, respectively, at the maximum power point $(M P P)$.

By construction, FF is less than one and measures how well the device maintains current extraction as bias is applied. 


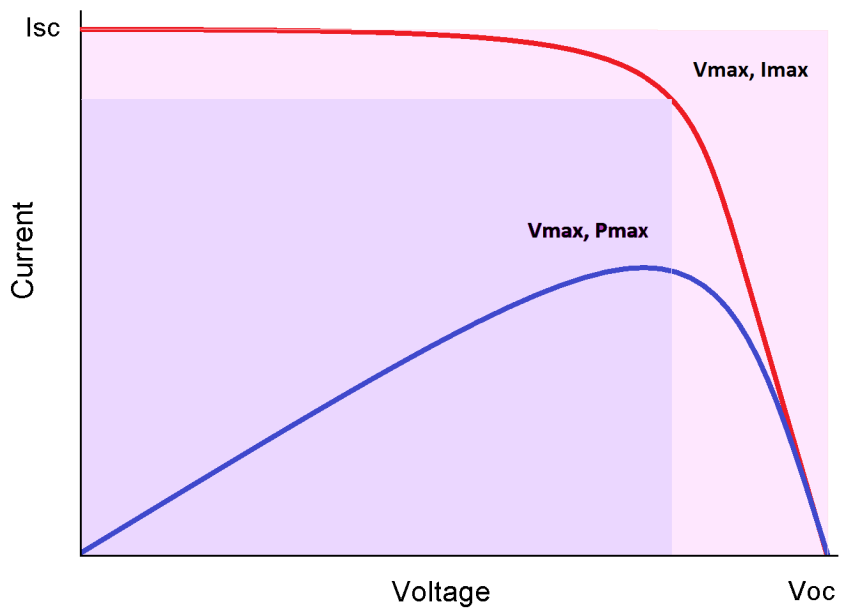

Figure 2.12: Graph of cell output current and power as a function of voltage. Graphically, the FF is a measure of the "squareness" of the solar cell and is also the area of the largest rectangle which will fit in the $I V$ curve.

Power Conversion Efficiency Power conversion efficiency $(P C E, \eta)$ is the most commonly used parameter to compare the performance between solar cells. $P C E$ is defined as the ratio of energy output from the solar cell to input energy from the sun. In addition to reflecting the performance of the solar cell itself, the efficiency depends on the spectrum and intensity of the incident sunlight and the temperature of the solar cell. Therefore, conditions under which efficiency is measured must be carefully controlled in order to compare the performance of one device to another. Solar cells are measured under the incident light described by the AM1.5 spectrum and with an irradiance of $I_{i n}=1000 \mathrm{~W} / \mathrm{m}^{2}$. Then, the efficiency of a solar cell is determined as the fraction of incident power which is converted to electricity and is defined as:

$$
\eta=\frac{P_{\max }}{I_{\text {in }}}=\frac{J_{S C} V_{O C} F F}{I_{\text {in }}}
$$

where $J_{S C}$ is the short-circuit current density in $m A / \mathrm{cm}^{2}, V_{O C}$ is the open-circuit voltage in volts, and $F F$ is the fill factor. 
Collection Efficiency Another figure of merit of some relevance is the collection efficiency, defined relative to both optical and recombination losses as an external collection efficiency

$$
\eta_{C}^{e x t}=\frac{I_{S C}}{I_{i n c}}
$$

where $I_{\text {inc }}$ is the maximum possible photocurrent that would result if all photons with $E>E_{G}$ created electron-hole pairs were collected.

It can also be defined with respect to recombination losses as the internal collection efficiency

$$
\eta_{C}^{i n t}=\frac{I_{S C}}{I_{g e n}}
$$

where $I_{g e n}$ is the light-generated current. This represents what the short-circuit current would be if every photon absorbed were collected and contributes to the short-circuit current. If there is no grid shadowing, no reflective losses and we suppose the solar cell has infinite optical thickness, then $I_{\text {gen }}=I_{\text {inc }}$.

It is easy to see that an efficient solar cell should have a high short-circuit current $I_{S C}$, a high open-circuit voltage $V_{O C}$ and a fill factor $F F$ close to one. Then the efficiency can be written as

$$
\eta=\frac{P_{M P}}{P_{i n}}=\eta_{\text {ideal }} \eta_{\text {photon }} F F \eta_{V} \eta_{C}^{i n t}
$$

where:

- $\eta_{\text {ideal }}$ is the ideal efficiency assuming the maximum energy that can be extracted from an absorbed photon is $E_{G}$

- $\eta_{\text {photon }}$ is the photon efficiency accounting for photons that are reflected, transmitted through or not absorbed in the solar cell and is expressed

- $\eta_{V}$ is the voltage efficiency defined by the ratio of the open-circuit voltage 
to the bandgap voltage.

\section{Efficiency limits}

The maximum efficiency $\left(\eta_{B C}\right)$ of a Carnot engine coupled to a blackbody absorber at temperature $T_{A}$, surrounded by an ambient with temperature $T_{0}$, with an emission source at temperature $T_{S}$ is given by

$$
\eta_{B C}=\left(1-\frac{\Omega_{e m i t}}{\Omega_{a b s}}\left(\frac{T_{A}}{T_{S}}\right)^{4}\right)\left(1-\frac{T_{0}}{T_{A}}\right)
$$

where $\Omega_{\text {emit }}$ and $\Omega_{a b s}$ are the solid angles of emission and absorption for the blackbody. The maximum Carnot efficiency is $85 \%$ for $T_{A}=2478 \mathrm{~K}, T_{S}=$ $5800 K, T_{0}=300 K$ and $\Omega_{e m i t}=\Omega_{a b s}=\pi[187,195]$, despite the fact that real $P V$ devices cannot approach this efficiency.

In [188], the authors derived the theoretical efficiency versus bandgap, obtaining a maximum $P C E$ of $30 \%$ for a bandgap of $1.1 \mathrm{eV}$. For the $A M 1.5 G$ spectrum, the maximum efficiency is $33 \%$ for a bandgap of 1.34 $e V[196,197]$.

An important loss mechanism in $P V$ devices is the loss of solar energy to the production of heat in the material, called thermalization. Not all of the energy from absorbed photons can be converted to useful energy: any energy above the conduction band edge is lost to heat, as carriers relax to their band edges, emitting phonons (lattice vibrations) in the process [187].

\subsubsection{Perovskite Bandgap Origin}

The $E_{g}$ of the lead halide perovskites is defined by the lead halide framework [39,198]. The $V B$ edge of the $M A P b I_{3}$ perovskite consists mainly of $I 5 p$ orbitals overlapping with the $P b 6 p$ and $6 s$ orbitals, and the $C B$ edge consists of $P b 6 p-I 5 s \sigma^{*}$ and $P b 6 p-I 5 p \pi^{*}$ hybridized orbitals. Similarly, 
bromide perovskites are defined by the $4 p$ orbitals of the $\mathrm{Br}$ atom and chloride perovskites by the $3 p$ orbitals of the $C l$ atom [199]. It has been proved that the $E_{g}$ of the perovskite can be tuned by stoichiometric adjustment of the halide precursors [190].

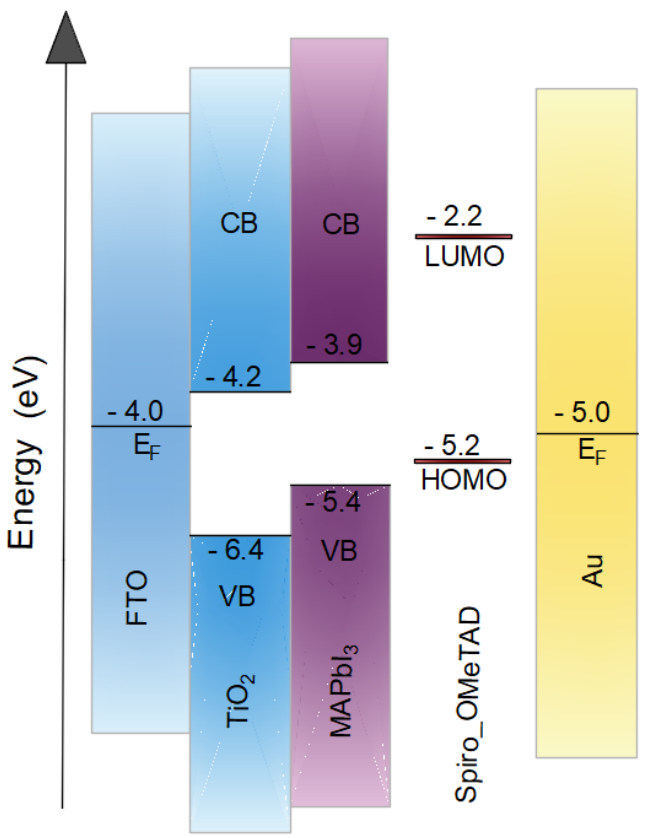

Figure 2.13: Energy band diagram of a typical $M A P b I_{3}$ perovskite solar cell.

The shift of the $E_{g}$ is due to the difference in the binding energies of the halide $p$ - orbitals. The $E_{g}$ of the perovskite is mostly mechanically affected by the choice of the cation $[200,201]$. Due to the size difference of the cation, the $\mathrm{Pb}-\mathrm{X}$ bond lengths and bond angles will be affected and, thereby, the valence electronic structure. Employing larger cations, such as the $F A^{+}$, results in a lower binding energy between the $P b$ and $X$, and hence lower $E_{g}$ of the $\mathrm{FAPbX}_{3}$ perovskite compared to the $\mathrm{CsPbX}$ perovskites. Even though a certain selection of cation, metal, and anions results in a $t$ between 0.8 and 
1, this does not necessarily mean that a stable perovskite material can be formed [190]. An energy band diagram of a typical $M A P b I_{3}$ perovskite solar cell is shown in Figure 2.13.

\subsubsection{Hysteresis in Current-Voltage Measurements}

The term hysteresis is derived from an ancient greek word meaning deficiency or lagging behind. Hysteresis relates to the history-dependent behavior of a system under test. It was first employed around 1890 by Sir James Alfred Ewing and it is commonly associated with ferromagnetic materials. When an external magnetic field is applied to a ferromagnetic material, the atomic domains align themselves with it. Even when the field is removed, part of the alignment will be retained: the material has become magnetized. If the magnetic field is then applied in different directions the magnetization of the material changes in a way that is dependent on the previous magnetization state, creating a hysteresis loop as shown in Figure 2.14 .

Similar history-dependent behavior has been observed in the measurement of solar cell current-voltage $(J-V)$ curves. $J-V$ sweep allows to assess the steady-state power output over a range of different bias levels. The bias is usually swept across a wide voltage range, covering at least the range from short-circuit to the open-circuit voltage. A small delay at each potential step is often incorporated to allow the current to settle to the steady state value.

In theory the measured $J-V$ curve should be independent of the sweep direction in order to be representative of steady state behavior [44]. However, it has been widely observed that perovskite solar cells present a substantial mismatch between the current density-voltage $(J-V)$ curves measured on forward scan (from short circuit to open circuit) and backward scan (from 
open circuit to short circuit).

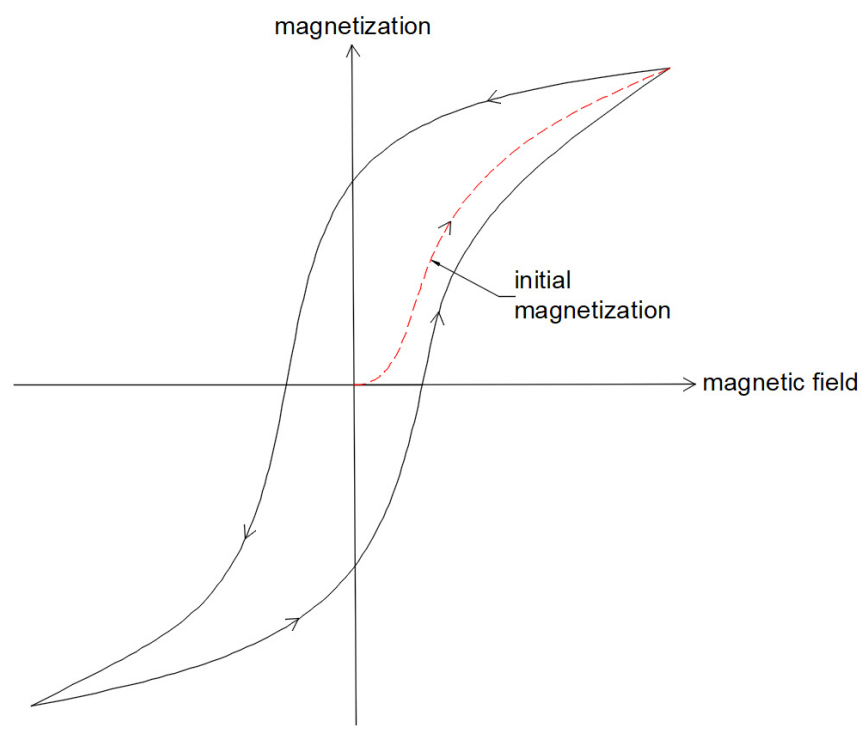

Figure 2.14: Example of a magnetic hysteresis loop for a ferroelectric material. The material is initially magnetized to a saturation value. Subsequent change in the magnetic field causes the magnetization to change relative to the previous condition (the history of system).

$J-V$ hysteresis is also found in dye-sensitized solar cells, organic thin film solar cells, and Si solar cells, when the voltage scan is too fast [202]. In devices made of perovskite, modification of the interface between the perovskite and the electron collecting layer apparently affects hysteresis. For instance, modification of $\mathrm{TiO}_{2}$ compact layer with $\mathrm{C60}$ [203] reduces hysteresis. Incorporation of $\mathrm{Zr}$ [204] and $\mathrm{Au}$ nanoparticles [205] in the $\mathrm{TiO}_{2}$ compact layer also reduces hysteresis. 


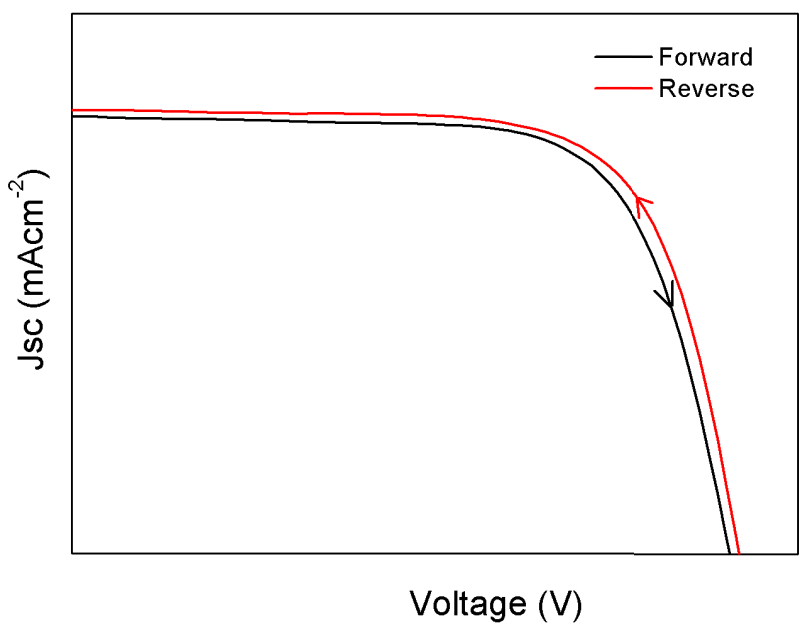

Figure 2.15: Example of hysteresis effect on a $J-V$ curve in perovskite solar cell.

Hysteretic $J-V$ curves imply that there is a clear difference in transient carrier collection at a given voltage during the forward and backward scan (see Figure 2.15). It is generally known that backward scan measures higher current than the forward scan, independent of scan sequence. This confirms that carrier collection is always more efficient during the backward scan. In general, carrier collection (or current) in the device depends on carrier generation, separation, and carrier transport in the bulk of the layers and transfer across different interfaces in the device. As carrier generation and separation are considered fast processes and depend only on illumination (not on the voltage scan) any difference in initial collection must be influenced by transport and/or transfer at the interfaces [44].

As carrier collection depends on the conductivity of perovskite and other layered materials and the connectivity at their interfaces, hysteresis is observed to be affected by many factors that can slightly change the characteristics of the layers in a perovskite device. Such anomalous hysteresis in perovskite cells 
is influenced by many factors such as device architecture, characteristics of metal oxide scaffold, thickness and morphology of perovskite films, properties of the electron, and hole conducting layers, and even the conditions of treatment before and during the measurement [44].

In order to understand the cause of hysteresis in $J-V$ curves of perovskite solar cells, different origin mechanisms to hysteresis have been presented. It has been proposed that the cause may be by ferroelectric polarization [206-210], ion migration [206,211], carrier dynamics at different interfaces or deeper trap states in the perovskite [206]. Although, actually there is no mechanism that can explain the phenomenon coherently. However, all the proposed mechanisms implicitly refer to altered carrier collection efficiency during the scans. As carrier collection depends both on transport in the bulk and transfer at the interfaces in the device, it is obvious that both the bulk properties (e.g., ferroelectric polarization, high density of mobile ions and trap states, etc.) and the interfacial contacts contribute to hysteresis.

Although understanding the mechanism of origin of hysteresis is doubtlessly the first step toward finding a solution to this anomalous behavior of perovskite solar cells, determining how hysteresis affects the actual cell performance is more important with regard to commercialization. Indeed, it holds more relevance to practical use of the solar cells [44]. The steady-state performance of cells operating at a constant voltage (or across an external load) is what matters for real use of any solar cell. As hysteresis is a transient phenomenon observed only upon voltage scan, it may not be considered a big problem as long as the device offers stable and reliable steady-state performance across a constant load over time. The only thing we need to know is the actual performance of devices showing hysteresis while operating at a constant voltage (at maximum power point). For devices showing 
hysteresis, the stabilized power output at MPP should be measured as the actual performance. Although, in most cases, the stabilized power output falls closer to the power calculated during the forward scan, it is sensitive to the structure and conditions of preparation [44]. 



\section{Chapter 3}

\section{Characterization Techniques}

\subsection{X-ray Diffraction}

$X$-ray diffraction $(X R D)$ is a common nondestructive analytical technique employed for phase identification of a crystalline material, in which the crystal atoms diffract an incident beam of $X$-rays into specific directions. $X R D$ allows determining the mono- and polycrystalline mineral phases of the materials by measuring their structural properties, such as the size of the grains, the epitaxial state, the composition of phase, preferential orientation and structural defects. The crystalline state is characterized by the triperiodic distribution in the space of an atomic motif. This ordered distribution constitutes the parallel and equidistant planes known as lattice planes $(h, k, l)$. Interreticular distances are of the order of 0.15 to $15 \AA$ and depend on the arrangement and diameter of the atoms in the crystal lattice. They are constants that characterize the crystal and are calculated using $X$-ray diffraction.

The incident $X$-ray beam interacts with the sample producing intense reflected $X$-rays by constructive interference when conditions satisfy Bragg's Law: 


$$
2 d_{h k l} \sin \theta=n \lambda
$$

where $\lambda$ is the wavelength of the incident $X$-rays, $\theta$ is the incident angle of the beam, $d_{h k l}$ is the spacing between the crystal lattice plane of atoms and $n$ is the order diffraction.

The grain size of the samples was evaluated from the $X R D$ line broadening using the Scherrer method:

$$
D=K \lambda / \beta \cos \theta
$$

where $D$ is the crystallite size, $\lambda$ is the wavelength, $k$ is a constant equal to $0.9, \beta$ is the peak width at half maximum and $\theta$ is the peak position.

Pawley fit was performed to fit the peak profiles of thin film perovskites. In order to solve a structure from powder data it is necessary to extract as many $h k l$ and intensity values as possible from the data set.

The factors $R p$ (profile factor)and $R w p$ (weighted profile factor) were used as numerical criteria to evaluate the fitting quality of the simulated to experimental data:

$$
\begin{aligned}
R w p & =100\left(\frac{\sum w_{i}\left(y_{i}^{o b s}-y_{i}^{c a l c}\right)^{2}}{\sum w_{i}\left(y_{i}^{\text {obs }}\right)^{2}}\right)^{\frac{1}{2}} \\
R p & =100\left(\frac{\sum\left|y_{i}^{\text {obs }}-y_{i}^{\text {calc }}\right|}{\sum\left|y_{i}^{\text {obs }}\right|}\right)
\end{aligned}
$$

where $y_{i}^{o b s}$ is the intensity of experimental profile for a given $2 \theta$ angle, $w i=\left(1 / y_{i}^{o b s}\right)$ is the weight of profile determination and $y_{i}^{c a l c}$ is the calculated intensity.

$X$-Ray diffraction patterns of perovskite films on ITO or FTO substrates 
were obtained by using a RIGAKU Ultima IV in the Bragg-Bentano configuration using $C u K_{\alpha}$ radiation $(\lambda=1.541841 \pm 0.002 \AA)$.

For perovskite materials, the $X R D$ technique helps to determine the crystal phase and to evaluate the crystallinity of the material. It is also possible to observe the formation of the perovskite as well as its degradation. Perovskite precursors, particularly the lead compounds, have a rather distinct $X R D$ pattern due to their crystallinity and are, therefore, easy to visualize from the perovskite diffraction pattern.

\subsection{Photoluminescence}

Photoluminescence $(P L)$ spectroscopy is a nondestructive analytical technique to study the electronic structure of materials. It is defined as the spontaneous emission of light from a material under optical excitation. The intensity and spectral content of the emitted photoluminescence provides information about the bandgap, the impurity levels and defects as well as recombination mechanisms.

Photoluminescence spectra have been recorded at low temperature using an $H E$-closed cryostat. $P L$ measurements were achieved using a $325 \mathrm{~nm}$ $H e-C d$ laser by a back-thinned $S i-C C D$ Hamamatsu detector.

\subsection{Atomic Force Microscope}

In the Atomic Force Microscope (AFM), a sharp point located at the end of a flexible lever travels the surface of a sample while maintaining a constant small interaction force. The scanning motion is performed by a piezoelectric scanner, and the peak/sample interaction is monitored by reflecting a laser on the back of the lever, which is collected in a photodiode detector. The 
photodiode is divided into 4 segments, and the voltage differences between the different segments determine precisely the changes in the inclination or amplitude of oscillation of the tip, which when in contact with the sample may cause surface modifications.

AFM measurements have been done with the Bruker Multimode 8 . Contact AFM, tapping AFM, phase Image, tunnel effect microscopy, magnetic forces, lift mode, lateral forces, force-distance measures, and electrochemistry are the working modes availables.

\subsection{Field Emission Scanning Electron Microscope}

The Field Emission Scanning Electron Microscope (FESEM) uses as a source of electrons a field emission cannon that provides very focused high and low energy electron beams, which improves the spatial resolution and allows working at very low potentials $(0.02-5 \mathrm{kV})$. This helps to minimize the loading effect on nonconductive specimens and to avoid damaging samples sensitive to the electronic beam.

The equipment that employed is the ULTRA 55 model of ZEISS, with the following implemented detectors: $S E 2$ secondary electron detector, secondary electron detector in len, detector of backscattered electrons $A s B$, backscattered electron detector in lens $E s B, X$-ray dispersive energy detector $(E D S)$ and $G E M I N I$ technology.

\subsection{Optical Spectroscopy}

The $U V$-visible spectroscopy is a nondestructive analysis method commonly used in analytical chemistry for the quantitative determination of transition metal ions, highly conjugated organic compounds, and biological 
macromolecules.

The Beer-Lambert law states that the absorbance of a solution is directly proportional to the concentration of the absorbing species in the solution and the path length. Therefore, $U V$-visible spectroscopy can be employed to determine the concentration of the absorber in a solution. The light intensity after it passes through a sample can be expressed as

$$
I(x)=I_{0} e^{-\alpha x}
$$

where $x$ is the distance traveled by the wave in the material, $I_{0}$ is the initial light intensity and $\alpha$ is the absorption coefficient, calculated by means of Lambert's formula

$$
T=A e^{-\alpha d}
$$

with $T$ being the transmittance, $d$ the thickness of the sample and $\alpha$ is the absorption coefficient. $\alpha$ verifies the following formula

$$
\alpha=\frac{1}{d} \ln \left(\frac{1}{T}\right)
$$

The optical bandgap $\left(E_{g}\right)$ of the samples can be estimated by using the Tauc equation

$$
(\alpha h v)^{2}=A\left(h v-E_{g}\right)
$$

where $h \nu$ is the photon energy, $\alpha$ is the absorption coefficient and $A$ is a constant. The optical properties, were carried out at room temperature using spectrometer Ocean Optics HR4000 equipped with a Si-CCD detector and an integrating sphere to collect specular and diffuse transmittance at room temperature. 



\section{Chapter 4}

\section{Synthesis and Bandgap}

\section{Tuning of $M A P b X_{3-x} Y_{x}$ \\ $(X, Y=I, B r, C l)$ Hybrid}

\section{Perovskites}

\subsection{Motivation}

Numerous studies have focused on material properties related to device performance, such as carrier mobilities, recombination lifetimes, excitonic properties and optical absorption [212]. One of the main problems of perovskite-based devices is the lack of long term stability to become scalable. With the aim to improve stability, research on the mixed halide perovskite $M A P b I_{3-x} C l_{x}$ have been carried out for photovoltaic applications [75, 213], derived from the pure halide perovskite $\mathrm{MAPbI}_{3}$ via apical substitution of the iodine atom with a chlorine atom.

Organolead halide perovskite materials also have excellent emission 
properties and have been used in photovoltaic applications as light absorbers for high energy photons [214] or can be employed to build efficient light emitters [215]. Solution processed crystalline $M A P b I_{3-x} C l_{x}$ films are able to convert $70 \%$ of the absorbed light into emitted light [216].

Recently, ultra-stable amplified spontaneous emission for solution-processed organic-inorganic perovskites $\mathrm{MAPbX}_{3}$, where $X=C l, B r, I$, have also been reported [217] paving the way for the realization of on-chip coherent light sources. Laser-light emission from perovskite crystals consisting of two-dimensional layers of metal halides, separated by organic materials, has also been reported [217].

It has been proved [55] that the bandgap of a perovskite can be tuned by varying the halide (i.e., bromide, iodide) composition of the perovskite precursor solution. High open-circuit voltages have been obtained by using the higher bandgap of $M A P b B r_{3-x} C l_{x}$, and these perovskites have been used in photovoltaic applications as a light absorber for high energy photons [214]. In [218] a bromide-iodide lead perovskite film, $M A P b I_{1.2} B r_{1.8}$ with an optical bandgap of $1.94 \mathrm{eV}$, that can be used for tandem cell applications with $\mathrm{c}-\mathrm{Si}$ cells, has been reported. However, it is important to establish whether these processes form solid solutions or if there is compositional inhomogeneity, which may introduce a range of local bandgaps reducing $P V$ performance. It has been reported that the $P L$ emission intensity and the average recombination lifetime strongly depends on the $\mathrm{Br}: \mathrm{Cl}$ ratio.

The longest recombination lifetime of $446 \mathrm{~ns}$ was found for $M A P b B r_{2.4} C l_{0.6}$ while the strongest $P L$ intensity was found for $M A P b B r_{2.25} C l_{0.75}[212]$. Depending on the excitation, $M A P b B r_{3-x} C l_{x}$ perovskites exhibit moderate to high $P L$ quantum efficiencies at room temperature. This moderate $P L$ efficiency at low excitation density has been 
attributed to the presence of defects causing nonradiative decay, meanwhile at high excitation density the defects are filled and radiative recombination becomes dominant [219].

The emission of the $M A P b B r_{3-x} C l_{x}$ perovskite has been tuned from 2.42 to $3.16 \mathrm{eV}$, allowing the construction of light emitting diodes operating at room temperature and emitting in green and blue ranges [220]. However, despite the outstanding photovoltaic and emission performances that have been obtained using $M A P b I_{3}$ and its related mixed perovskites, the formation of mixed halide perovskites remains poorly investigated.

In this chapter, an investigation on optical and structure properties of pure $\mathrm{MAPbX}_{3}(X=I, \mathrm{Br}, \mathrm{Cl})$ perovskites and their related $\mathrm{I} / \mathrm{Br}$ and $\mathrm{Br} / \mathrm{Cl}$ mixed halide lead methylammonium perovskites is presented.

Pure $I, B r$ and $C l$ lead methylammonium perovskites have been synthesized from suitable solutions and then mixed perovskites were obtained by mixing pure perovskites in proper proportions. The halide ratio in the thin film perovskites follows the same ratio used in liquid precursors. Crystalline structure, as well as the relationship between the absorption and emission of pure and mixed halide perovskites, as a function of the $I: B r$ and $B r: C l$ ratio in the material has been studied. It has been found, that the exact composition of the final compound reproduces the stoichiometry of the precursor solution, therefore the optical absorption and emission properties can be optimized through composition tuning.

The photoluminescence properties and the recombination behavior of the solution processed $M A P b X_{3-x} Y_{x}(X, Y=I, B r, C l ; x=0,1,2,3)$ thin films depend on different ratios of $I: B r$ and $B r: C l$. 


\section{$4.2 \quad$ Experimental}

\subsubsection{Synthesis of Pure Perovskite Powders}

$\mathrm{MAPbX}_{3}(X=I, \mathrm{Br}, \mathrm{Cl})$ perovskite crystal powders were synthesized by mixing an equimolar ratio of $0.30 \mathrm{~mol}$ of $\mathrm{CH}_{3} \mathrm{NH}_{2}$ (33\% in methanol from Sigma Aldrich) and hydrohalide acid $H X(X=I, B r, C l)$. The mixture was stirred for 2 hours in a $250 \mathrm{~mL}$ round-bottom flask, kept in an ice bath $\left(0^{\circ} \mathrm{C}\right)$. Subsequently, this mixture was heated to $100^{\circ} \mathrm{C}$ for 30 minutes, and then a solution of $0.03 \mathrm{~mol}$ of $\mathrm{Pb}\left(\mathrm{NO}_{3}\right)_{2}$, previously dissolved in $50 \mathrm{~mL}$ of distilled water, was added dropwise to the hot $M A X_{3}(X=I, B r, C l)$ solution under vigorous stirring.

After this process, a precipitate of crystalline powders with different colors (black for iodide, orange for bromide and white for chloride) took place. The remaining solution was then left to cool until $40^{\circ} \mathrm{C}$ and filtered. The crystalline powders were washed several times with absolute ethanol, diethyl ether and then dried under vacuum. In particular, $M A P b I_{3}$ powders were filtered on vacuum with the solution above $40^{\circ} \mathrm{C}$ to avoid decomposition [64]. The synthesis described before complies with the following equation:

$$
\mathrm{CH}_{3} \mathrm{NH}_{2}+3 \mathrm{HX}+\mathrm{Pb}\left(\mathrm{NO}_{3}\right)_{2} \longrightarrow \mathrm{CH}_{3} \mathrm{NH}_{3} \mathrm{PbX}_{3}+2 \mathrm{NO}_{3}^{-}+2 \mathrm{H}^{+}
$$

\subsubsection{Synthesis of Thin Film Perovskites}

Iodine and bromine crystalline powders were dissolved at $45 \% \mathrm{wt}$ in dimethylformamide $(D M F)$ solution and chloride perovskite was dissolved in dimethylsulfoxide $(D M S O)$. The different mixed halide perovskites $\operatorname{MAPbX}_{3-x} Y_{x}(X, Y=I, B r, C l ; x=0,1,2,3)$ were prepared by mixing the respective pure precursor solutions $\mathrm{MAPbX}_{3}(X=I, B r, C l)$ at $45 \%$ wt in the ratios 1:2 and 2:1, respectively. All solutions, three for pure perovskite and 
four for mixed halide perovskites were then deposited onto FTO substrates by spin coating or by dipping. Mixed perovskite containing iodides and chlorides were not considered in this study.

\subsection{Results and Discussion}

\subsubsection{X-ray Diffraction}

The methods used to synthesize pure perovskite powders $M A P b X_{3}$ for $X=I, B r, C l$ were successful for the preparation of high quality samples in crystal form.

Figure 4.1 shows the powder $X$-ray diffractogram patterns for $M A P b X_{3}$ $(X=I, B r, C l)$ in tetragonal symmetry.

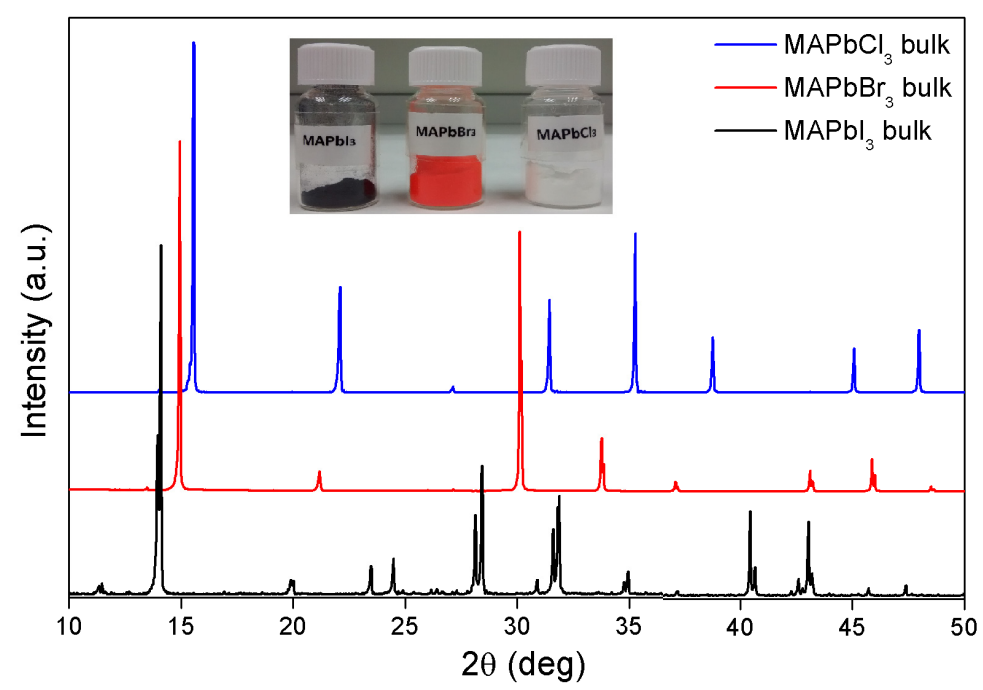

Figure 4.1: $X$-Ray diffractograms of $\mathrm{MAPbX}_{3}(X=I, B r, C l)$ perovskite bulk. Includes images of single crystal powder using the Weber method [64]. 


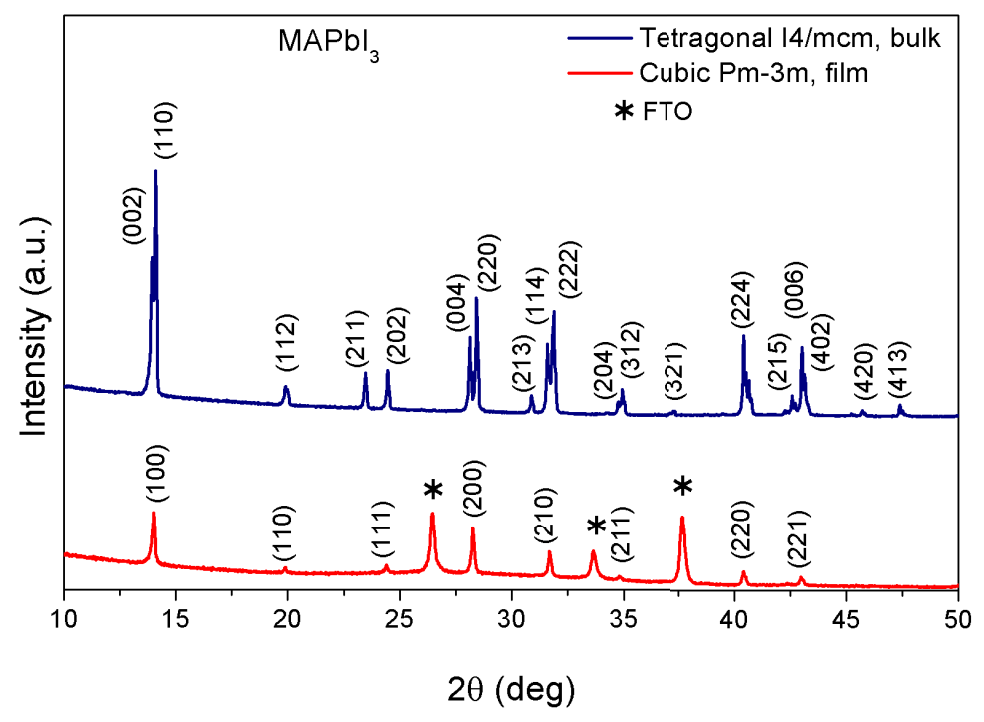

Figure 4.2: Comparison of the powder and thin film $X$-ray diffractograms for $\mathrm{MAPbI}_{3}$.

Figure 4.2 shows a comparison of the powder and thin film $X$-ray diffractograms for $\mathrm{MAPbI}_{3}$ in tetragonal $I 4 / \mathrm{mcm}$ and cubic $P m \overline{3} m$ symmetry, respectively. The (211) and (213) reflections in the powder diffractogram are sufficiently separated from Bragg reflections expected in cubic symmetry, in agreement with [39].

Figures 4.3 and 4.4 show the powder and thin film $X$-ray diffractograms for

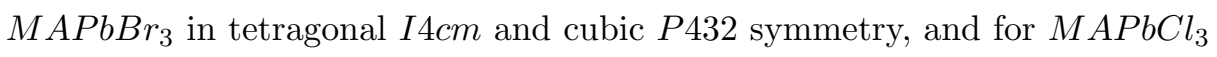
in tetragonal $P 4 / n$ and cubic $P 432$ symmetry, respectively. 


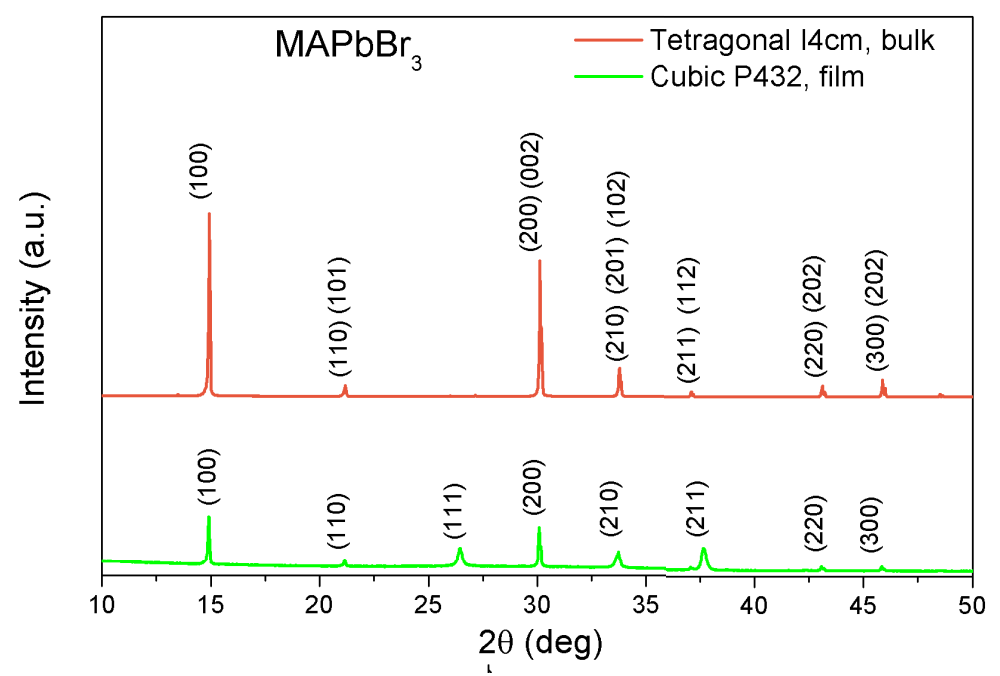

Figure 4.3: Comparison of the powder and thin film $X$-ray diffractograms for $\mathrm{MAPbBr}_{3}$.

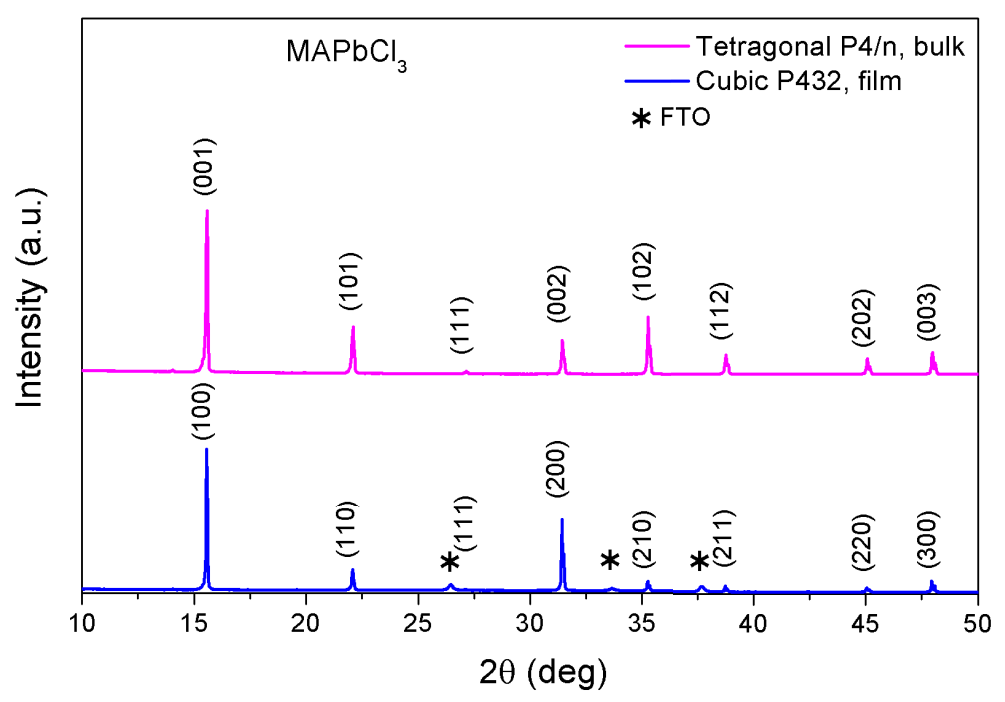

Figure 4.4: Comparison of the powder and thin film $X$-ray diffractograms for $\mathrm{MAPbCl}_{3}$.

Figure 4.5 shows the $X$-ray diffractograms for $M A P b I_{3}$ and $M A P b B r_{3}$ thin films and the combinations with bromide $\left(\mathrm{MAPbI}_{2} \mathrm{Br}\right.$ and $\left.\mathrm{MAPbIBr} \mathrm{A}_{2}\right)$. 
All thin film diffractograms correspond to the same cubic structure and the most intense diffraction peak located below $15^{\circ}$ corresponds to (100) diffraction plane, and the peaks located at about $20^{\circ}, 30^{\circ}$ and $34^{\circ}$ are related to (110), (200) and (210) diffraction planes, respectively.

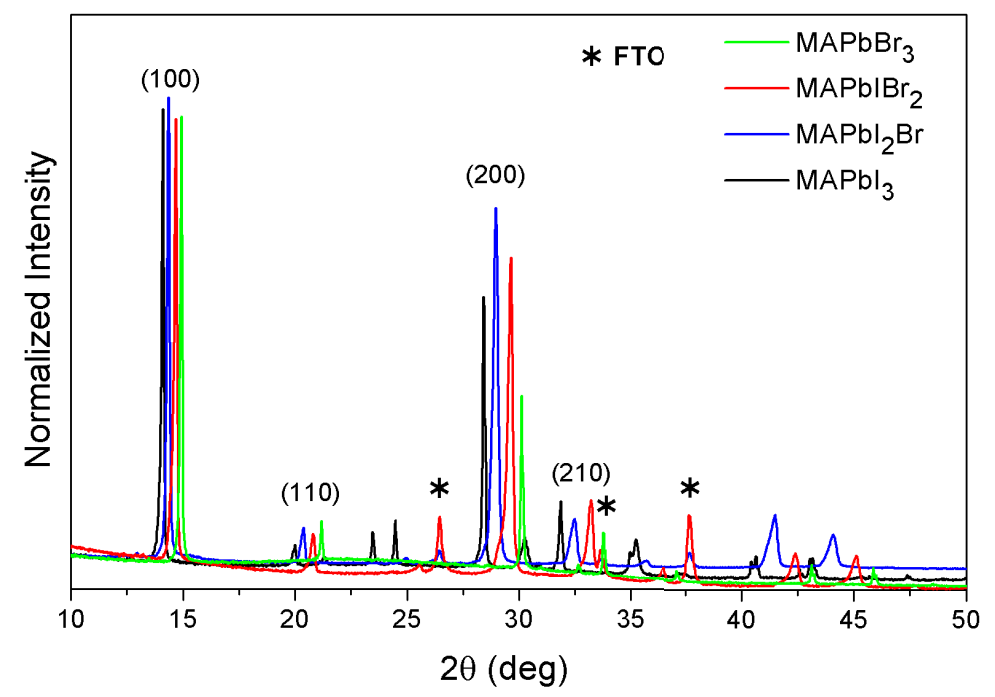

Figure 4.5: $X$-ray diffractogram for $M A P b I_{3-x} B r_{x}(x=0,1,2,3)$ thin films.

Three diffraction peaks corresponding to the FTO substrate and located at $26.5^{\circ}, 33.7^{\circ}$ and $37.7^{\circ}$ are also observed. Figure 4.6 shows the shift of the peak position according to the following trend: the lower the average size of the halides in the perovskite, the higher the position of the diffraction peak, or vice versa. Figure 4.7 shows the linear relation between the peak position and the bromide content $x$. 


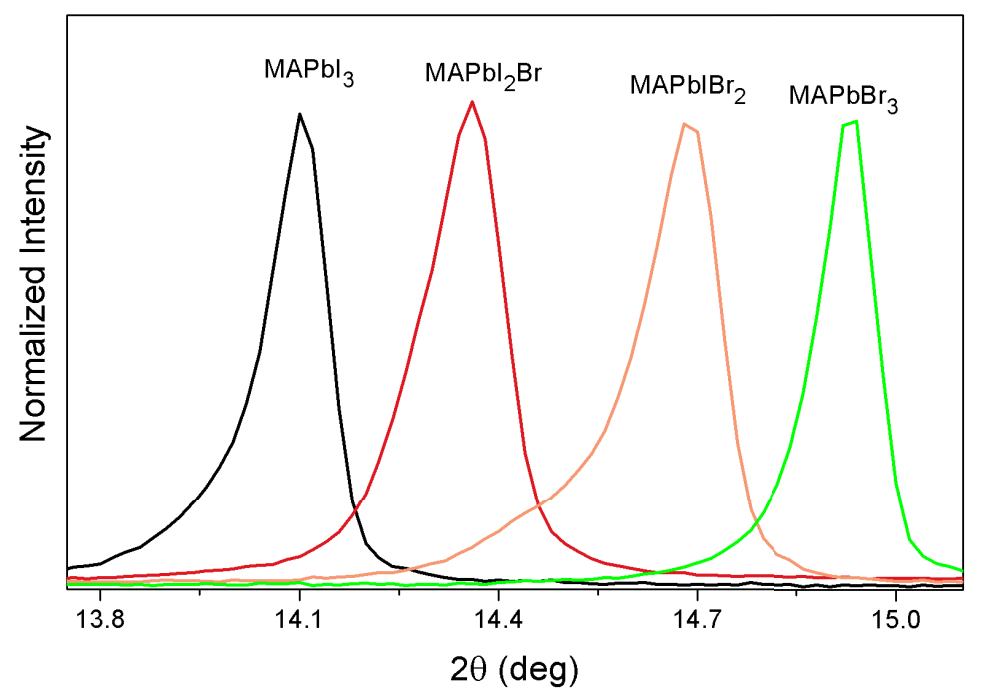

Figure 4.6: $X$-ray diffractogram for $\mathrm{MAPbI}_{3-x} \mathrm{Br} r_{x}$ magnified in the region of the strongest plane intensity (100) located between $13.8^{\circ}$ and $15.0^{\circ}$.

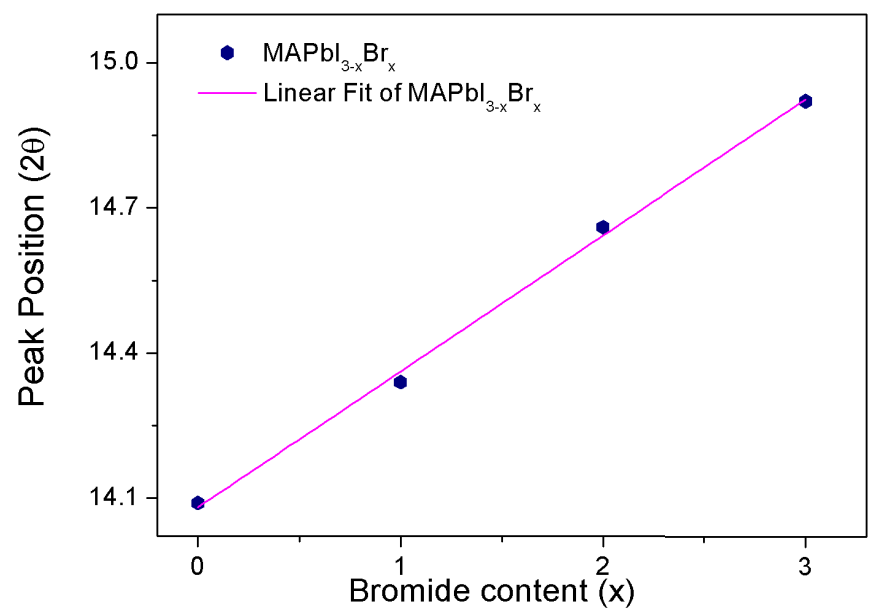

Figure 4.7: Peak position of $M A P b I_{3-x} B r_{x}$ in terms of $x$ content.

The lattice constants were calculated by fitting the whole pattern using $J A D E$ software. It was found that the lattice constant follows Vegard's law [221], which states that lattice constants vary linearly with the ratio between the two halide components of the thin films (see Figure 4.8). 


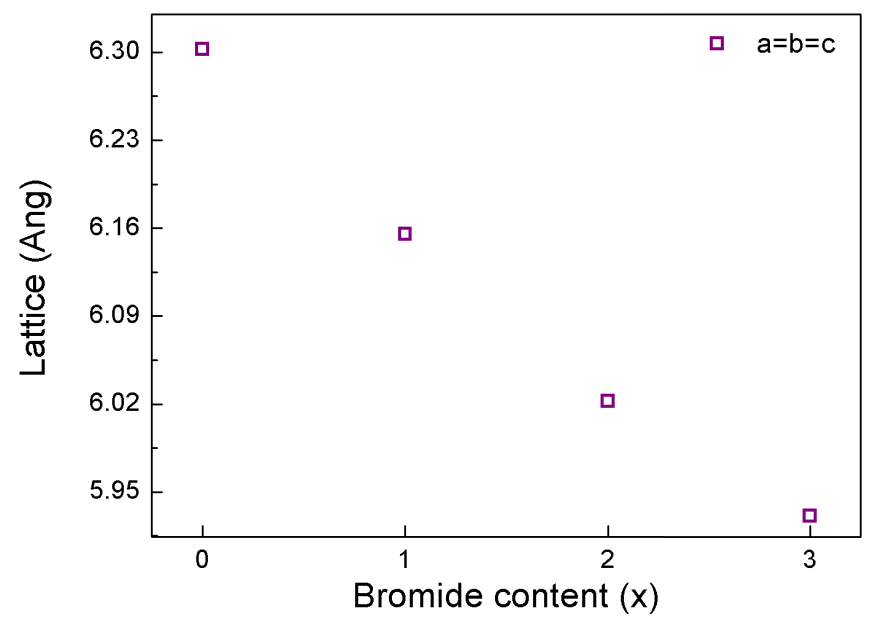

Figure 4.8: Lattice parameter represented as a function of bromide content.

Figure 4.9 shows the $X$-ray diffractograms for pure $M A P b B r_{3}$ and $\mathrm{MAPbCl}_{3}$ thin films and their corresponding mixtures for $x=1,2$. The intensity of $X R D$ peaks has been normalized for the intensity of the most intense (100) peak. As mentioned before, pure and mixed $\mathrm{Br}$ and $\mathrm{Cl}$ perovskite thin films exhibit a cubic structure belonging to the spatial group P432. Figure 4.10 displays the shift of the position of the diffraction peaks to higher angles when the average size of halides contained in the thin film decreases, which means that the distance between diffraction planes is reduced due to the lower average size of halides.

In Figure 4.11, the peak position as a linear function of chloride content $x$ can be seen. The lattice constant, calculated as mentioned before, follows the Vegard's law, and varies linearly with the ratio between the two halides components of the thin films, as shown in Figure 4.12.

Concerning the iodide and chloride mixed halide perovskite, we were not able to synthesize layers with the same $I: C l$ ratio, as we did for $B r: I$ and $\mathrm{Br}: \mathrm{Cl}$ mixed halides. In all attempts to synthesize $M A P b I_{3-x} C l_{x}$ thin films, the $C l$ content was very low, far from the desired 1:2 or 2:1 ratios, in agreement 
with the literature.

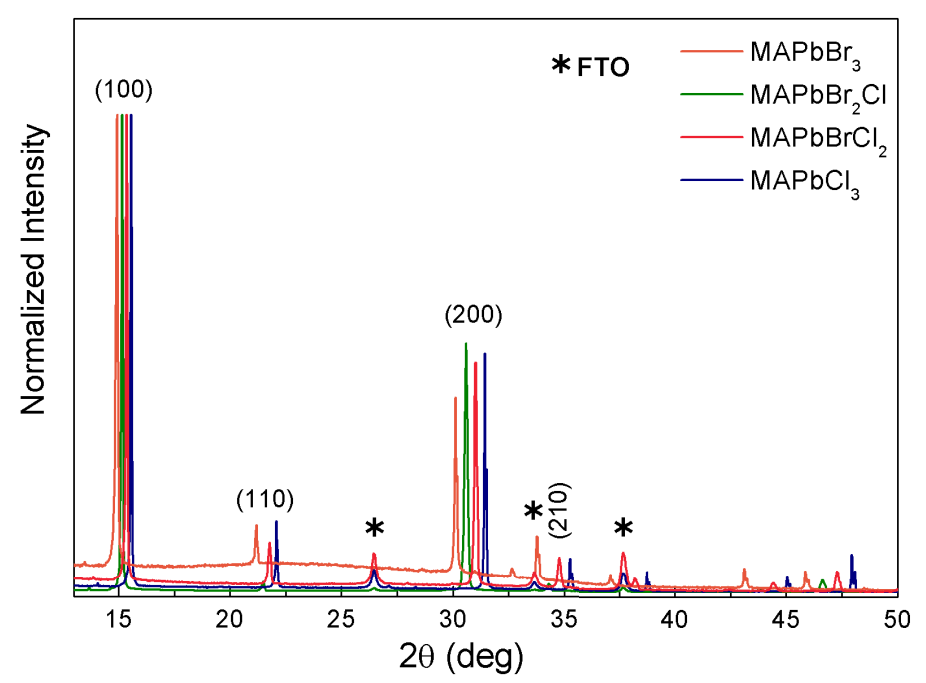

Figure 4.9: $X$-ray diffractogram for $\mathrm{MAPbBr}_{3-x} C l_{x}(x=0,1,2,3)$ thin films.

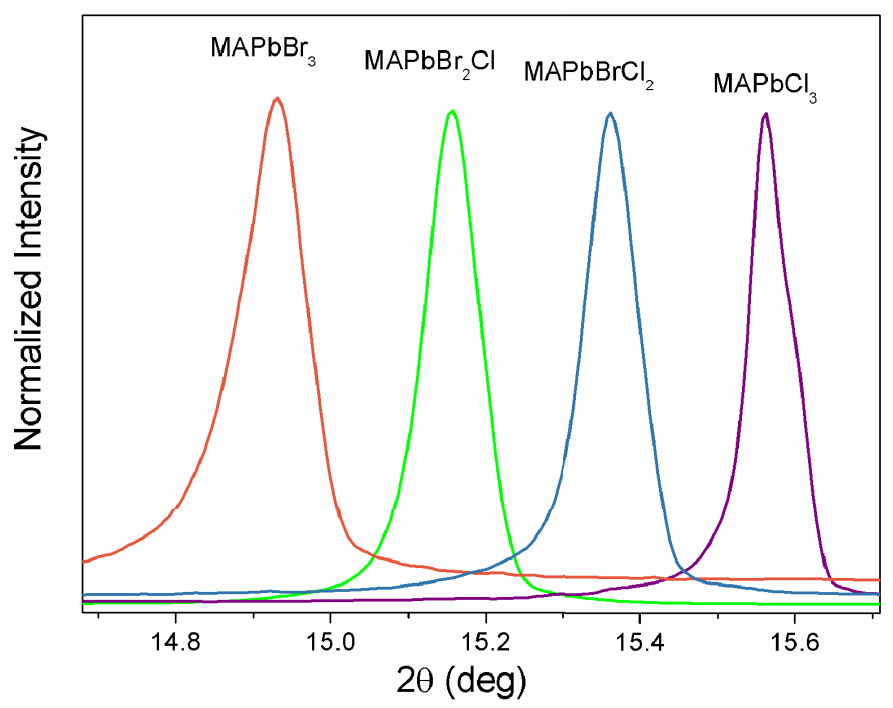

Figure 4.10: $X$-ray diffractogram for $\mathrm{MAPbBr}_{3-x} C l_{x}$ magnified in the region of the strongest plane intensity (100) located between $14.6^{\circ}$ and $15.8^{\circ}$. 


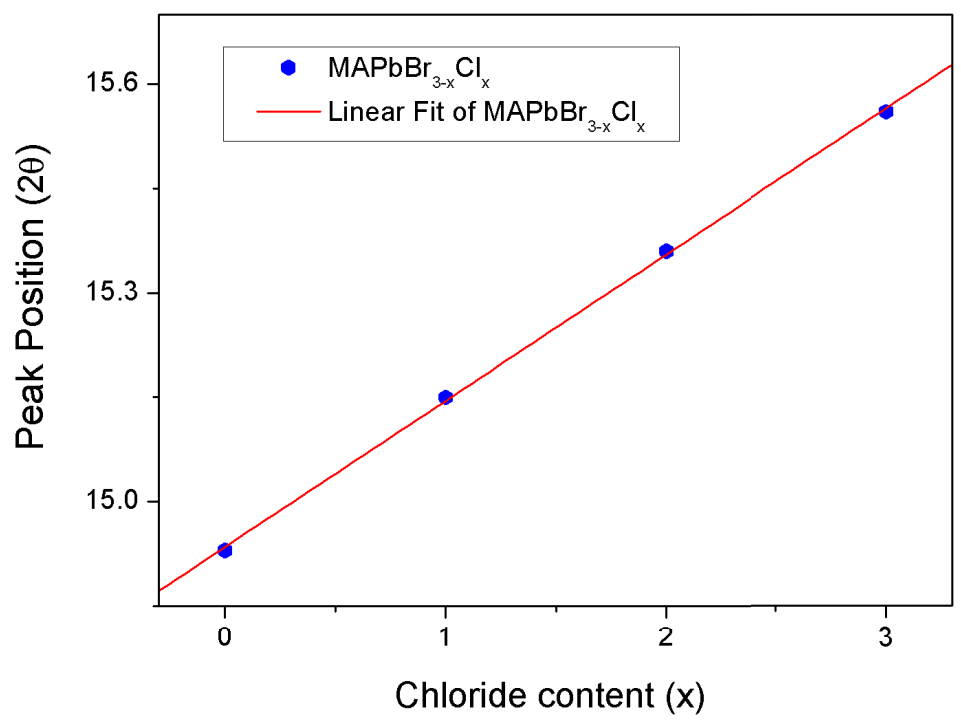

Figure 4.11: Peak position of $M A P b B r_{3-x} C l_{x}$ in terms of $x$ content.

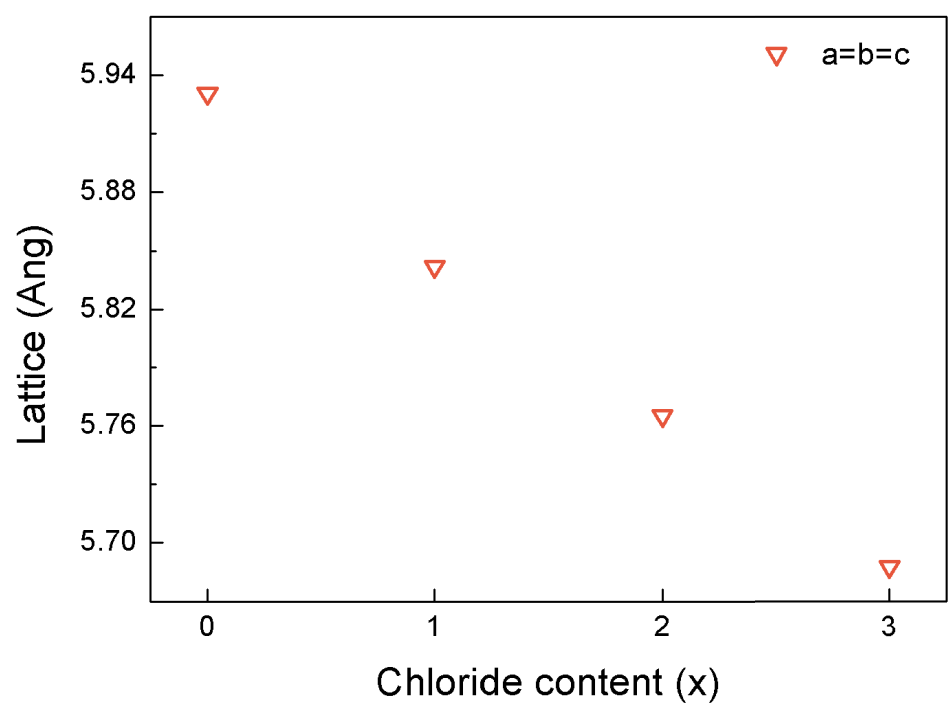

Figure 4.12: Lattice parameter of $\mathrm{MAPbBr}_{3-x} \mathrm{Cl}_{x}$ as a function of chloride content.

As mentioned in Chapter 2, it has been demonstrated, experimentally and theoretically, that the formation of continuous solid phase $M A P b I_{3-x} C l_{x}$ is 
actually not alloyed and that chloride incorporation into $\mathrm{MAPbI}_{3}$ is possible only at a relatively low concentration, so that it could be classified as a dopant agent rather than alloy [54]. However, the role of chloride is still being reviewed, and recent research has shown that chloride addition primarily acts as a crystallization retardant.

\subsubsection{Absorbance}

Figure 4.13 shows the absorbance for $M A P b X_{3}$ thin film perovskites, where $X=I, B r, C l$, and their mixtures $\mathrm{MAPbI}_{2} \mathrm{Br}, \mathrm{MAPbIBr}_{2}, \mathrm{MAPbBr} \mathrm{Cl}_{2} \mathrm{Al}$ and $\mathrm{MAPbBrCl}_{2}$. The main features of the absorption spectra are the existence of a sub-bandgap absorption tail, followed by a strong rise in absorption, which corresponds to excitonic absorption, and then a transition from the valence band to the conduction band. When excitonic absorption dominates the absorption spectrum, the classical relationship between the absorption coefficient $(\alpha)$ and the bandgap energy $\left(E_{g}\right)$, commonly used for calculating the bandgap of a direct semiconductor, is expressed as:

$$
\left(\alpha_{E}\right)^{2}=A\left(E-E_{g}\right)
$$

where $E$ is the photon energy and $A$ is a constant, is no longer valid and Elliott's equation has to be used [222]. The different widths exhibited for the different perovskites complicate the calculation of the real bandgap, thus, herein, the onset of the absorption edge will referred to. The absorption edge ranges from the lowest value, $1.66 \mathrm{eV}$ for $\mathrm{MAPbI}_{3}$, increases when decreasing the average size of the halides contained in the film, reaching $2.65 \mathrm{eV}$ for $M A P b B r_{3}$ and, its highest value, $3.37 \mathrm{eV}$, for $M A P b C l_{3}$. As expected, the lower size of the halogen anion in the perovskite thin films results in a shift of the absorption edge to higher energies. 


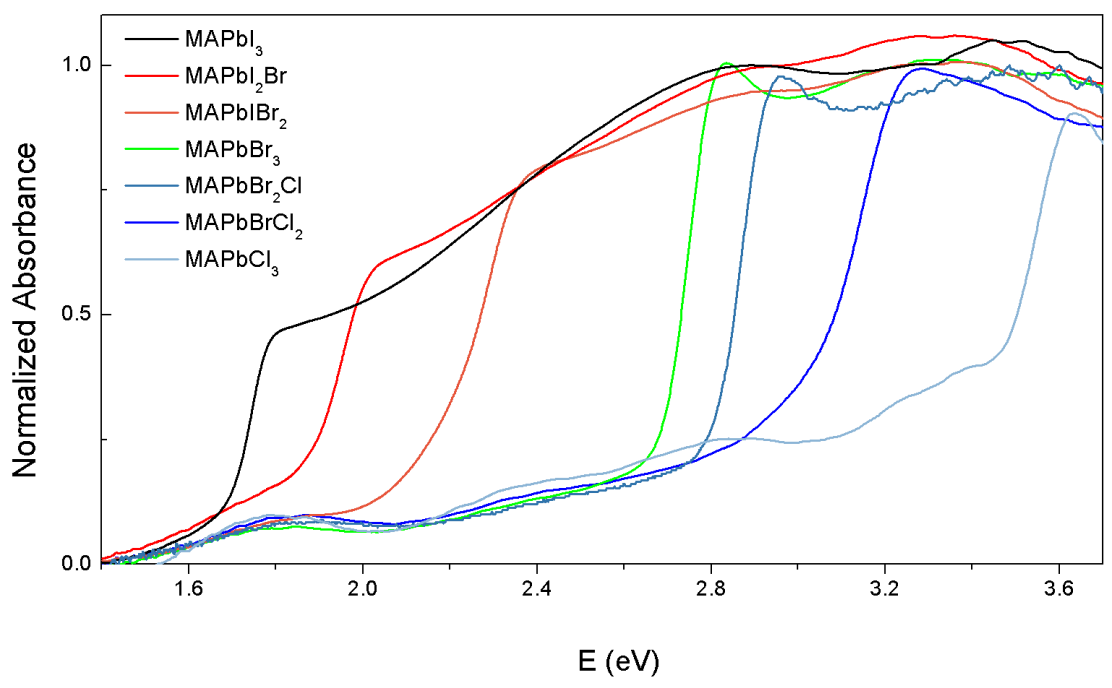

Figure 4.13: Absorbance for $M A P b I_{3-x} B r_{x}$ and $M A P b B r_{3-x} C l_{x}(x=$ $0,1,2,3)$ thin films.

The sub-band absorption exhibited for most perovskite thin films shown here could be related to optical transitions between the conduction or valence bands, and energy levels located inside the gap, or to the incomplete coating of the substrate, equivalent to the presence of holes in the films and that should be observed in the transmittance spectra. However, this possibility cannot be inferred from the transmittance spectra obtained, therefore we believe that the sub-bandgap absorption is related to transition involving intra-gap energy levels.

This hypothesis agrees with the features observed for the photoluminescence spectra. Table 4.1 displays the absorption edge at room temperature for the different perovskites in energy and in wavelength, whose values were calculated from the onset of the absorbance, shown in Figure 4.13. 
Table 4.1 Absorption edge for $M A P b I_{3-x} B r_{x}$ and $M A P b B r_{3-x} C l_{x}$ and the most intense $P L$ peaks.

\begin{tabular}{|c|c|c|c|c|}
\hline \multirow[t]{2}{*}{ Sample } & \multicolumn{2}{|c|}{$\begin{array}{c}\text { Absorption edge } \\
\text { at } 300 K\end{array}$} & \multicolumn{2}{|c|}{$\begin{array}{c}P L \text { peak position } \\
\text { at } 10 \mathrm{~K}\end{array}$} \\
\hline & $(n m)$ & $(e V)$ & $(n m)$ & $(e V)$ \\
\hline $\mathrm{MAPbI}_{3}$ & 746 & 1.66 & 780 & 1.58 \\
\hline$M A P b I_{2} B r$ & 685 & 1.81 & 725 & 1.71 \\
\hline$M A P b I B r_{2}$ & 590 & 2.10 & 618 & 2.00 \\
\hline$M A P b B r_{3}$ & 468 & 2.65 & 544 & 2.27 \\
\hline $\mathrm{MAPbBr} r_{2} \mathrm{Cl}$ & 448 & 2.77 & 514 & 2.41 \\
\hline$M A P b B r C l_{2}$ & 420 & 2.95 & 462 & 2.68 \\
\hline$M A P b C l_{3}$ & 368 & 3.37 & 398 & 3.11 \\
\hline
\end{tabular}

\subsubsection{Photoluminescence}

Figure 4.14 shows the photoluminescence spectra recorded at $10 \mathrm{~K}$ for the different pure $\mathrm{MAPbX}_{3}(X=I, B r, C l)$ thin film perovskites and their mixtures considered in this study. $P L$ emission energy depends on the halide components of the thin film, covering all the wavelengths of the visible spectrum. The lowest energy (highest wavelength, $790 \mathrm{~nm}$ ) is observed for $\mathrm{MAPbI}_{3}$, while the highest energy (lowest wavelength, $398 \mathrm{~nm}$ ) is observed for $\mathrm{MAPbCl}_{3}$. The wavelength position for $\mathrm{MAPbBr}_{3}$ is located in between $(550 \mathrm{~nm})$. 


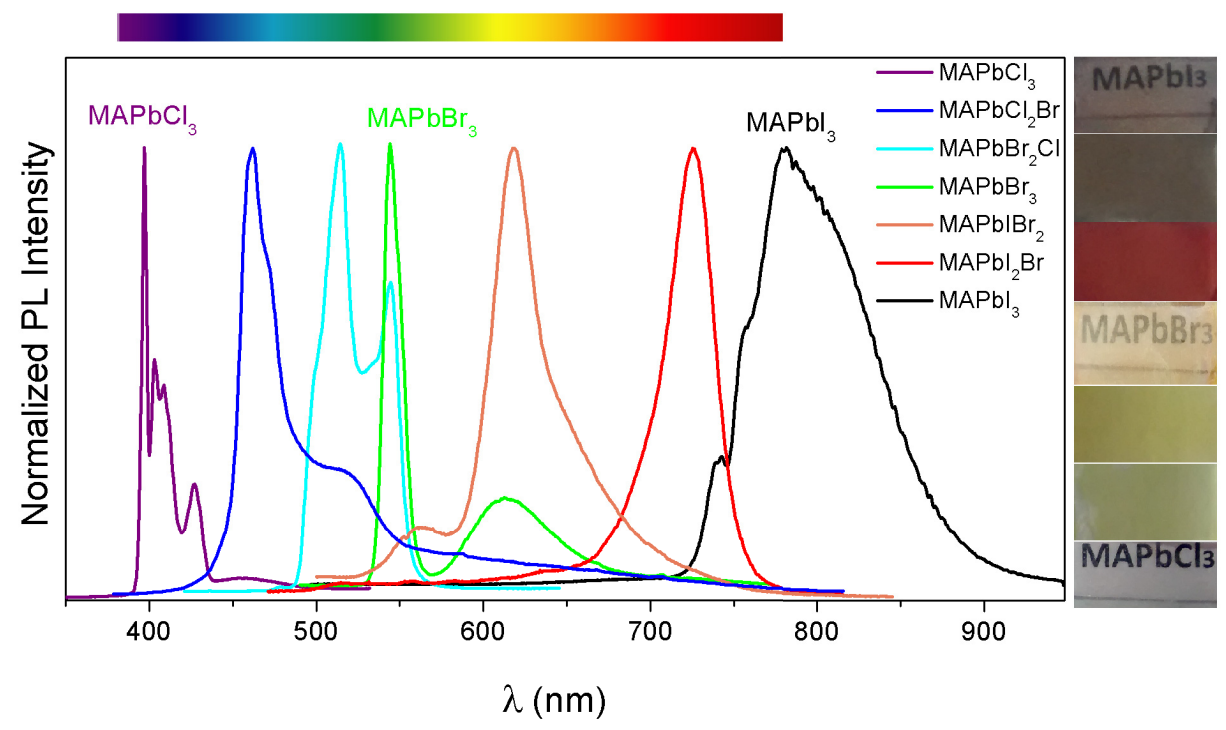

Figure 4.14: $P L$ spectra for $M A P b I_{3-x} B r_{x}$ and $M A P b B r_{3-x} C l_{x}$ (x= $0,1,2,3)$ thin films recorded at $10 \mathrm{~K}$. On the right, an image of the $M A P b I_{3-x} B r_{x}$ and $M A P b B r_{3-x} C l_{x}$ thin films.

In most cases the shape of the photoluminescence spectra contains several peaks, which means that, apart from inter-band transitions, other radiative transitions between the valence and/or conduction bands and some energy levels located inside the bandgap are involved. The component of $P L$ spectra with the highest energy corresponds to transitions from the conduction band to the valence band and the lower energy components of the $P L$ spectra involve energy levels inside the bandgap, which is in agreement with the sub-bandgap absorption mentioned before.

The wavelength position of the maximum of the photoluminescence emission is always shifted to higher wavelengths when compared to the onset of the absorption edge. 


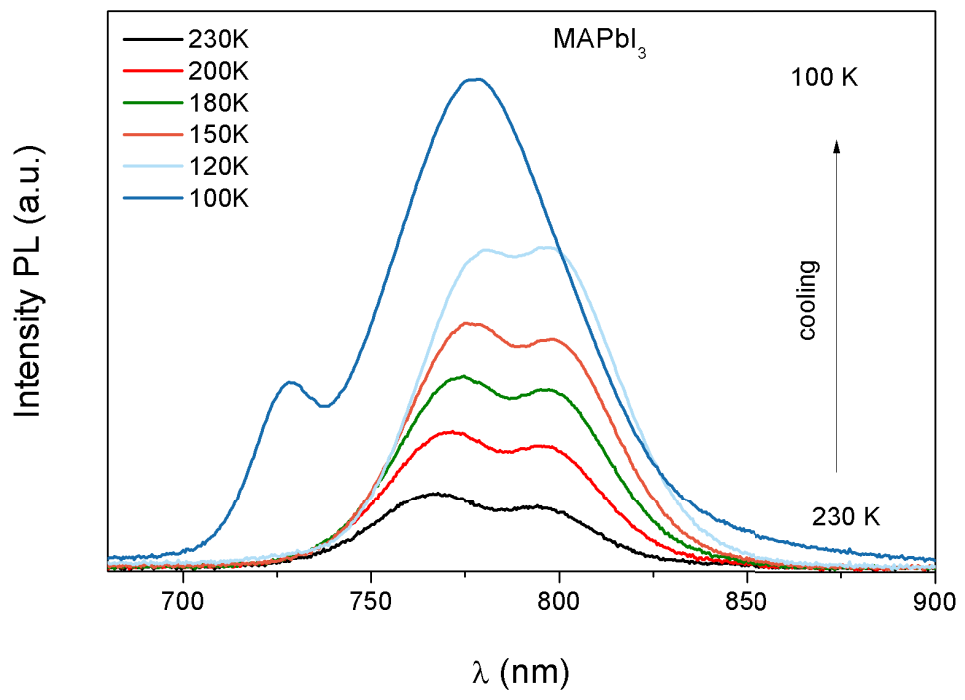

Figure 4.15: $P L$ spectra for $M A P b I_{3}$ in cooling.

The high intensity of $P L$ emission suggests that most decay transitions are radiative, and nonradiative decay is negligible. Since the radiative recombination dominates, it can be assumed that most energy levels inside the bandgap correspond to shallow levels and electron-hole pairs, formed during optical excitation, recombine radiatively emitting photons. The amount of deep levels inside the bandgap is very low and, as a result, both pure and mixed perovskites exhibit high luminescent efficiency. Table 4.1 shows the energy position and related wavelength for the most intense $P L$ peaks for the different $M A P b X_{3-x} Y_{x}(X, Y=I, B r, C l$ and $x=0,1,2,3)$ perovskite thin films. The energy position of $P L$ peaks is always red shifted with respect to the onset of the absorption edge.

Figure 4.15 shows the $P L$ spectra for $M A P b I_{3}$ recorded in cooling, in which it can be seen a shift to lower wavelengths and an increase in the intensity of the peak, indicating a change of phase around $120 \mathrm{~K}$. Figure 4.16 shows the $P L$ spectra in heating, displaying a change of phase around $160 \mathrm{~K}$, where the peaks 
shift to higher wavelengths and their intensity decreases when temperature increases. These changes in intensity and position are in agreement with the $D S C$ plot shown in Figure 4.17. The asymmetry of the $D S C$ plot could be indicative of the existence of a transient intermediate phase.

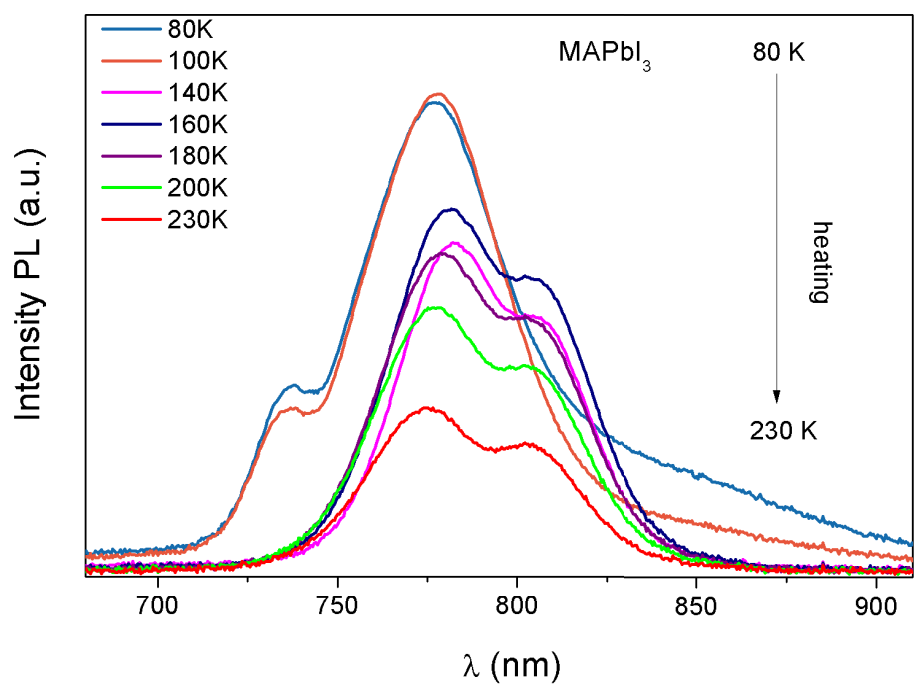

Figure 4.16: $P L$ spectra for $\mathrm{MAPbI}_{3}$ in heating.

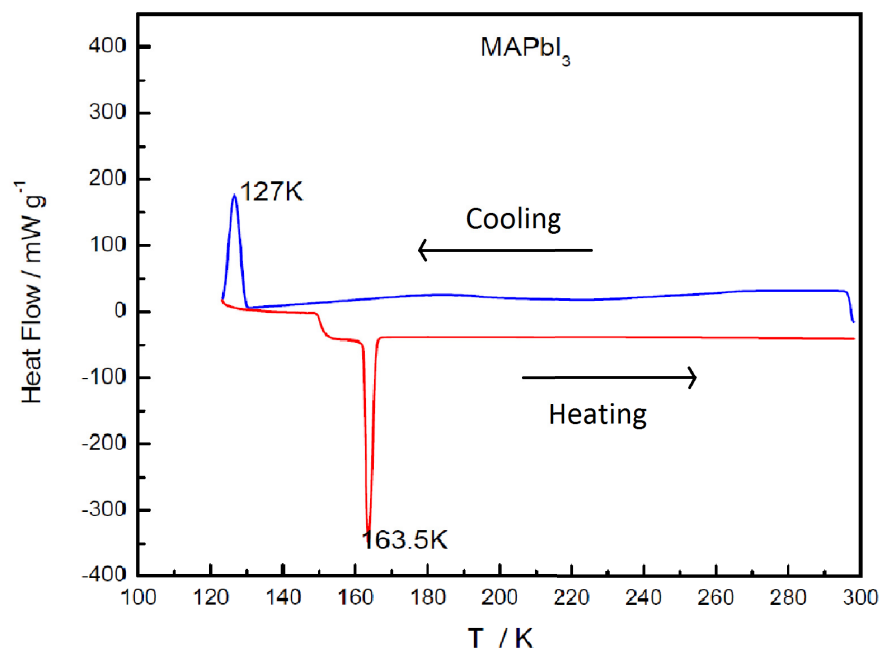

Figure 4.17: $D S C$ plot $M A P b I_{3}$. 
Figure 4.15 shows the $P L$ spectra for $M A P b I_{3}$ recorded in cooling, in which it can be seen a shift to lower wavelengths and an increase in the intensity of the peak, indicating a change of phase around $120 \mathrm{~K}$. Figure 4.16 shows the $P L$ spectra in heating, displaying a change of phase around $160 K$, where the peaks shift to higher wavelengths and their intensity decreases when temperature increases. These changes in intensity and position are in agreement with the $D S C$ plot shown in Figure 4.17. The asymmetry of the $D S C$ plot could be indicative of the existence of a transient intermediate phase.

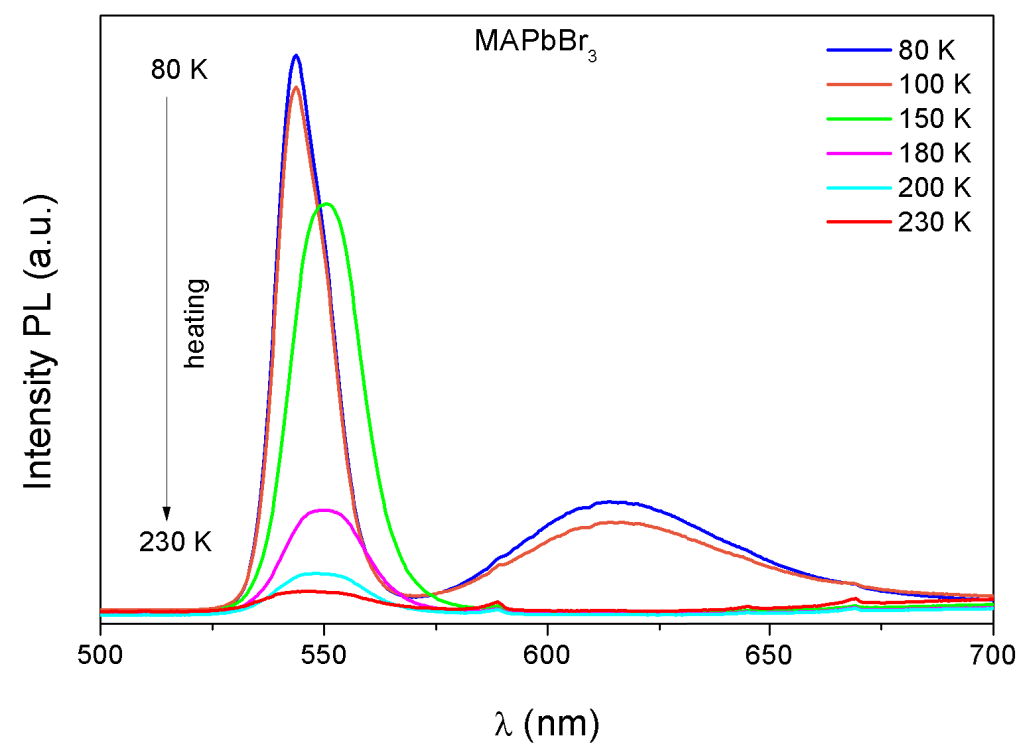

Figure 4.18: $P L$ spectra for $M A P b B r_{3}$ in heating.

$P L$ spectra recorded in heating for $M A P b B r_{3}$, see Figure 4.18, shows a shift to higher wavelengths and a decrease in the intensity of the peak, indicating, also, a change of phase at about $150 \mathrm{~K}$. Figure 4.19 shows the $D S C$ plot, being in agreement with the $P L$ data. Moreover, the asymmetry of the $D S C$ plot could indicate the existence of a transient intermediate phase. 


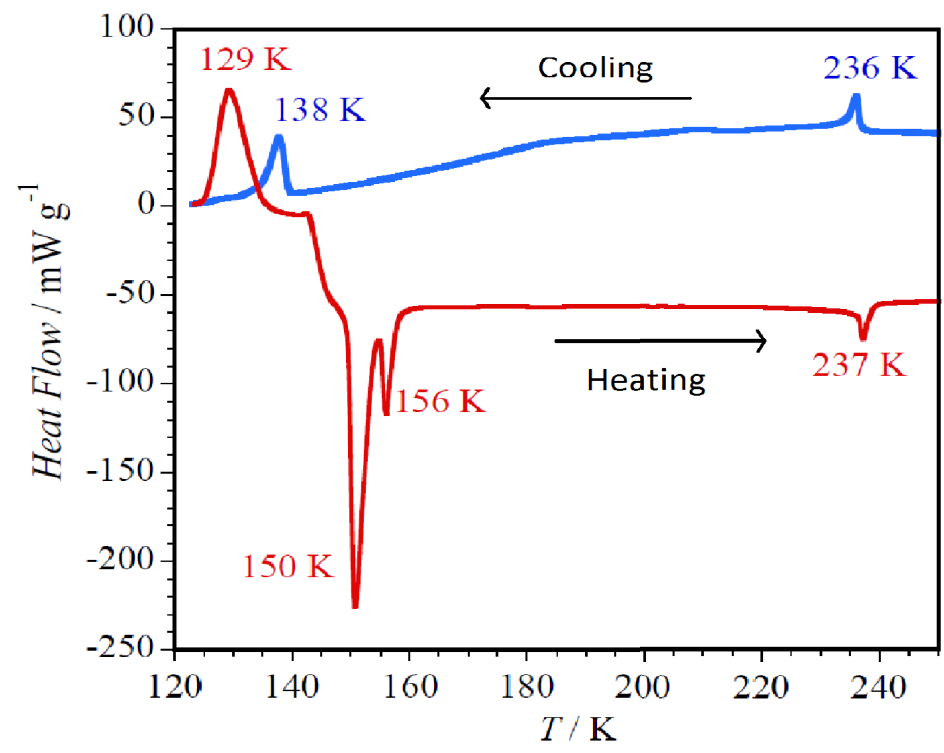

Figure 4.19: $D S C$ plot of $M A P b B r_{3}$.

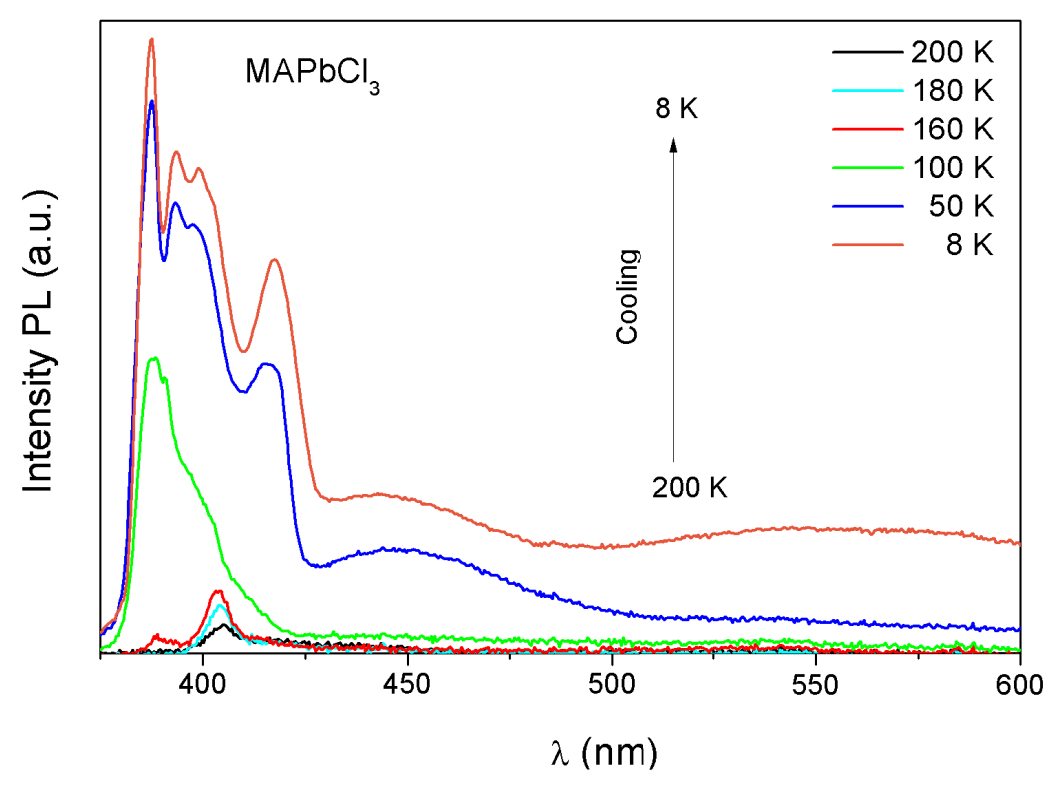

Figure 4.20: $P L$ spectra for $M A P b C l_{3}$ in cooling.

Finally, the $P L$ spectra for $M A P b C l_{3}$ recorded in cooling can be seen 
in Figure 4.20, where a shift to lower wavelengths and an increase in the intensity of the peak can be observed for temperatures between 160 and 100 $K$, indicating a change of phase. Furthermore, once $50 K$ has been reached, the intensity has further increased, and the peak has unfolded towards higher wavelengths, suggesting the existence of another change of phase. The first observed change of phase in $P L$ spectrum is corroborated by $D S C$ plots (Figure 4.21). The second change of phase can not be appreciated in the $D S C$ plot, since the minimum shown temperature is $150 \mathrm{~K}$.

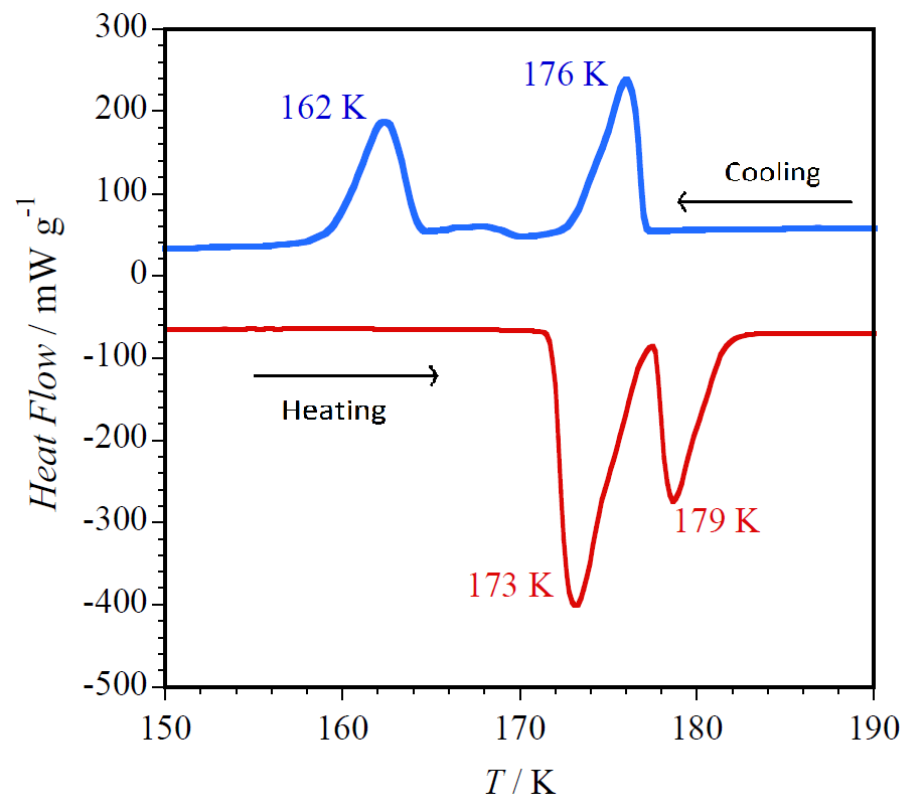

Figure 4.21: $D S C$ plot of $M A P b C l_{3}$.

\subsubsection{FESEM Analysis}

In order to study the morphology of perovskite powders and thin films, a FESEM analysis was carried out. Figure 4.22 shows a comparison between pure $\mathrm{MAPbX}_{3}$ with $X=I, B r, C l$ perovskite powders and thin films. 


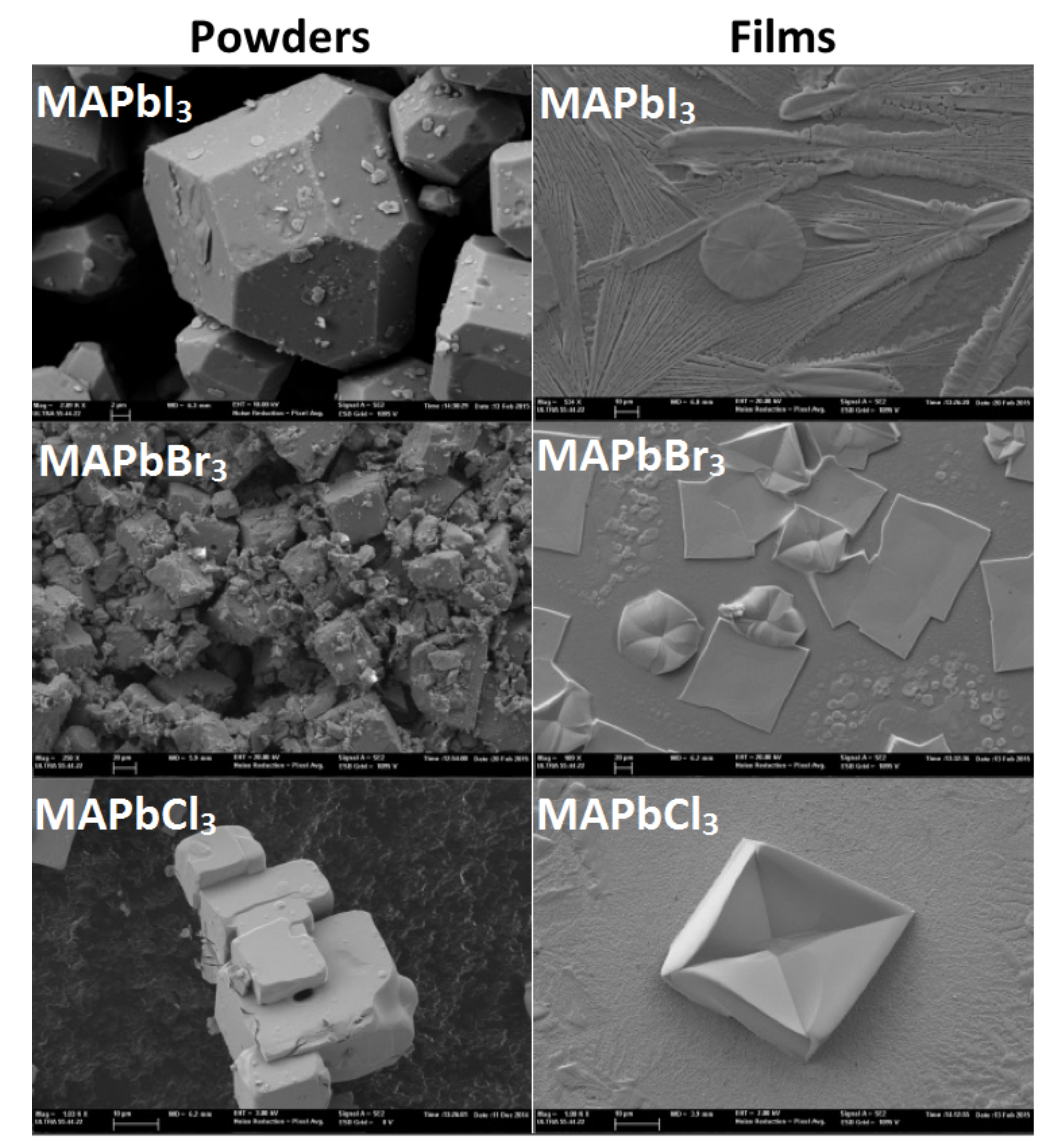

Figure 4.22: FESEM images of pure $\mathrm{MAPbX}_{3}$ powder and thin film, when $X=I, B r, C l$.

As it can be seen, both powders and thin films show different morphologies depending on the composition. Also, a significantly different morphology can be appreciated between powders and thin films for each perovskite composition. With respect to the powders, larger grains are easily observed for $\mathrm{MAPbI}_{3}$ and $\mathrm{MAPbCl}_{3}$ than that the observed for the $M A P b B r_{3}$ powders. On the other hand, thin films are not homogeneous nor uniform. The $\mathrm{MAPbI}_{3}$ film presents an irregular combined needle- and plate- shaped morphology and, the $\mathrm{MAPbBr}_{3}$ and $\mathrm{MAPbCl} l_{3}$ films show different crystal morphologies and sizes. 


\subsection{Conclusion}

Methylammonium lead halide perovskites with different halides (iodide, bromide and chloride) and methylammonium lead halides mixtures ( $I: B r$ and $B r: C l$ ), prepared by mixing pure methylammonium lead halide perovskites in the desired proportions, were synthesized from methylamine, lead nitrate and the corresponding hydroX acid $(X=I, B r, C l)$ precursors and deposited as thin films onto FTO substrates by spin coating or dipping.

$X$-ray diffractograms for $M A P b X_{3-x} Y_{x}$ films for $X, Y=I, B r, C l$ and $0 \leq x \leq 3$ reported in this chapter crystallized in cubic phase irrespective of the $X$ component and $x$ content, but the position of the peaks shifted according to the halide component and content in the films. Furthermore, the powders and films of all synthesized mixed halide thin film perovskites reached the expected stoichiometry, as inferred from elemental analysis. In perovskite mixed halides combining $I$ and $C l$, the chloride content was so low that it should be considered as a dopant agent rather than a mixture.

The absorption edge of thin film perovskites can be tuned all along the visible spectrum from 368 to $746 \mathrm{~nm}$. The bandgaps of film $M A P b X_{3-x} Y_{x}$ perovskites were inferred from transmittance spectral measurements. It was found that the bandgap for thin film $M A P b X_{3}$ perovskites is about 1.66, 2.65 and $3.37 \mathrm{eV}$ for $X=I, B r, C l$, respectively, whilst reaching intermediate values for mixed $M A P b X_{3-x} Y_{x}$ perovskites. Low temperature $P L$ emission can also be tuned along the visible spectrum from 400 to 800 $n m$, approximately. $P L$ emissions are centered at 1.58, 2.27 and $3.11 \mathrm{eV}$ for

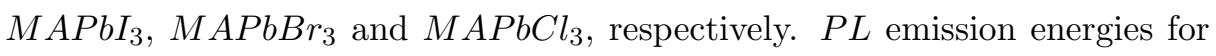

mixed halides perovskites are located in intermediate values. As a general rule, it was observed that the center of the $P L$ emission at $10 \mathrm{~K}$ is red shifted with respect to the absorption edge measured at room temperature. 



\section{Chapter 5}

\section{Role of the Chemical}

\section{Substitution in the Structural}

\section{and Luminescence Properties}

of the Mixed Halide

Perovskite $M A P b I_{3-x} B r_{x}$

$(0 \leq x \leq 1)$ thin films

\subsection{Motivation}

As mentioned in the previous chapters, despite the outstanding photovoltaic and emission performance reported, $\mathrm{MAPbI}_{3}$ perovskite materials are still not stable enough to become fully scalable. It has been proved that $\mathrm{MAPbI}_{3}$ degrades under humid conditions, and then forms $\mathrm{PbI}_{2}$ at higher temperatures, due to the loss of $M A I$ [223]. 
Some studies have found that $\mathrm{MAPbI}_{3}$ is more sensitive to moisture and humidity than $\mathrm{MAPbBr}_{3}[224,225]$, however, the absorption of $M A P b B r_{3}$ is not comparable to that of $\mathrm{MAPbI}_{3}$. Hence, some researchers focused on the preparation of mixed halide perovskite $M A P b I_{3-x} B r_{x}$ and $M A P b I_{3-x} C l_{x}$. The substitution of iodine by chloride or bromide atoms leads to a significant improvement of perovskite stability [55,226]. Although, there are numerous studies on the growth of mixed halide bromine perovskites, their formation process remains poorly investigated.

In order to better understand how to optimize the chemical composition of $\mathrm{MAPbI}_{3-x} \mathrm{Br}_{x}$ to meet the requirement of different applications, an investigation on the processed solutions, $X$-ray structural analyses and optical properties of the $M A P b I_{3-x} B r_{x}$ thin films are reported in this section.

\subsection{Experimental Procedure}

$\mathrm{MAPbI}_{3}$ and $\mathrm{MAPbBr}_{3}$ powders were first synthesized by reacting 0.30 mol of hydrohalide acid $H X(X=I, B r)$ (in water, Sigma Aldrich) with 0.30 mol of $\mathrm{CH}_{3} \mathrm{NH}_{2}$ (33\% in methanol, Sigma Aldrich) in a round-bottomed flask at $0^{\circ} \mathrm{C}$ for $2 \mathrm{~h}$ with stirring. The mixture was heated to $100^{\circ} \mathrm{C}$ for 30 minutes, and then, when the mixture is still hot, a solution of $0.03 \mathrm{~mol}$ of $\mathrm{Pb}\left(\mathrm{NO}_{3}\right)_{2}$ previously dissolved in $50 \mathrm{~mL}$ of distilled water, was added dropwise under vigorous stirring. The crystalline perovskite powders precipitate showing different colors: $M A P b I_{3}$ with black color and $M A P b B r_{3}$ with orange color. The remaining solution was left to cool-down and was then filtered. The obtained powders were washed several times with absolute ethanol and diethyl ether and were then dried under vacuum.

The precursor solutions were prepared by dissolving $M A P b I_{3}$ and $M A P b B r_{3}$ powders at $45 \%$ wt dimethylformamide $(D M F)$ solution. The 
mixed perovskite solutions were prepared by varying the iodide/bromide concentration. The mixed solutions were stirred for $3 h$ to ensure solution homogeneity. Thin film perovskites were then produced by spin coating at a speed of $3000 \mathrm{rpm}$ for $30 \mathrm{~s}$ or dipping. Finally, the samples were annealed for $1 \mathrm{~h}$ at $100^{\circ} \mathrm{C}$ and then kept in vacuum to avoid their degradation.

The synthesis of $M A P b I_{3}$ and $M A P b B r_{3}$ perovskite powders can be described by the following equations:

$$
\begin{gathered}
\mathrm{CH}_{3} \mathrm{NH}_{2}+3 \mathrm{HI}+\mathrm{Pb}\left(\mathrm{NO}_{3}\right)_{2} \rightarrow \mathrm{CH}_{3} \mathrm{NH}_{3} \mathrm{PBI}_{3}+2 \mathrm{NO}_{3}^{-}+2 \mathrm{H}^{+} \\
\mathrm{CH}_{3} \mathrm{NH}_{2}+3 \mathrm{HBr}+\mathrm{Pb}\left(\mathrm{NO}_{3}\right)_{2} \rightarrow \mathrm{CH}_{3} \mathrm{NH}_{3} \mathrm{PbBr}_{3}+2 \mathrm{NO}_{3}^{-}+2 \mathrm{H}^{+}
\end{gathered}
$$

\subsection{Results and Discussion}

\subsection{1 $X$-ray Analysis}

Figure 5.1 shows the $X$-ray diffraction patterns of $M A P b I_{3-x} B r_{x}$ with different bromide fractions. As the bromide content is increased, the diffraction profiles are shifted towards higher angles, resulting from the decrease of lattice spacing due to the partial substitution of the larger iodine atom (ionic radius $2.2 \AA$ ) with a smaller bromide atom (ionic radius $1.96 \AA$ ). The pure $M A P b I_{3}$ sample presents several diffraction profiles matching that expected for the tetragonal perovskite $I 4 / \mathrm{mcm}$, specifically the peak at $23.46^{\circ}$, associated with the (211) planes, disappeared at the composition $x=0.4$; out this range a cubic phase similar to the $M A P b B r_{3}$ compound having a space group $P m \overline{3} m$ was formed. The formation of the tetragonal $\mathrm{MAPbI}_{3}$ is in good agreement with previous reports in the literature $[46,47,227,228]$.

The reflections $(004)_{T}$ and $(220)_{T}$ of the $M A P b I_{3}$ phase merged with the $(200)_{c}$ direction of the cubic phase, also the $(002)_{T}$ and $(110)_{T}$ were combined 
in the peak centered at about $14.12^{\circ}$ with a full width at half maximum (FWHM) of $0.20^{\circ}$ (Figure 5.2). The existence of these double peaks with high FWHM values, is due to the fact that the planes $(00 l)$ and $(h k 0)$ are related to $c$ and $a=b$ axes, hence a slight difference in the $d_{h k l}$ spacing for each peak.

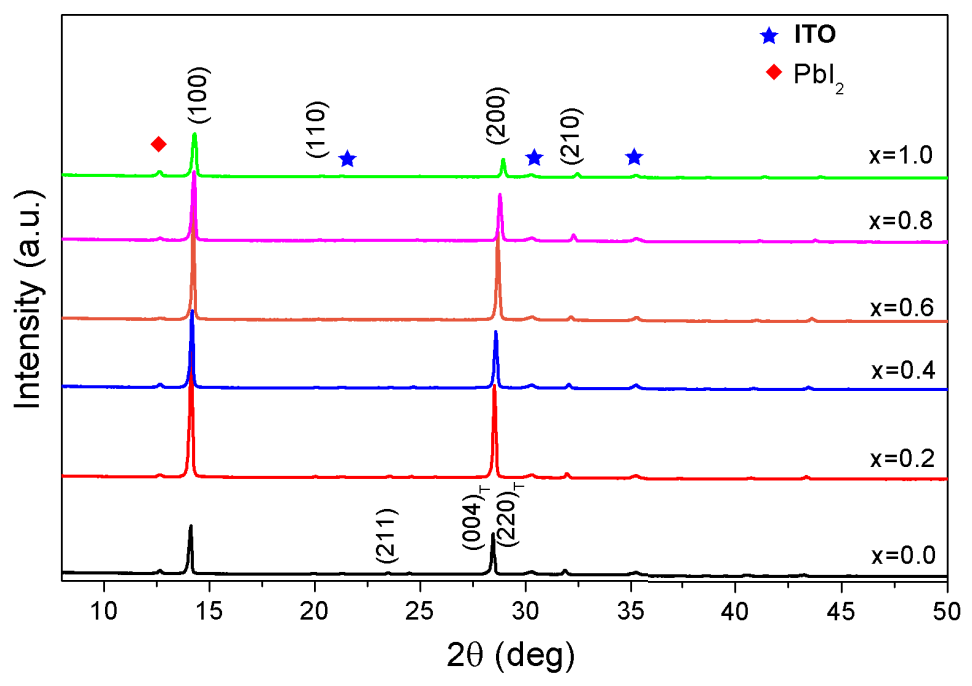

Figure 5.1: $X R D$ of $M A P b I_{3-x} B r_{x}(0 \leq x \leq 1)$ thin films.

In the $\mathrm{MAPbI}_{3}$ tetragonal phase, there was a strong preferential orientation along the $(h k 0)$ planes. It should be noted that the diffractograms indicated the presence of $\mathrm{PbI}_{2}$ diffraction peak at $12.64^{\circ}$ with a small intensity. This peak has been often identified in thin film $X$-ray diffraction, which may be an indicator of a slight decomposition of the samples [39]. These results suggest that the presence of the impurity phase $\mathrm{PbI}_{2}$ is due to the solution interaction $M A P b B r_{3} / \mathrm{MAPbI}_{3}$ that leaves a small amount of $M A P b I_{3}$ unreacted, thus leaving a corresponding amount to form $\mathrm{PbI}_{2}$. However, as can be appreciated in Figure 5.1, the formation of $\mathrm{PbI}_{2}$ was negligible and the perovskite peaks were dominant. The peaks located at about $21^{\circ}, 30^{\circ}$ and $35^{\circ}$ correspond to 
the $I T O$ glass substrate.

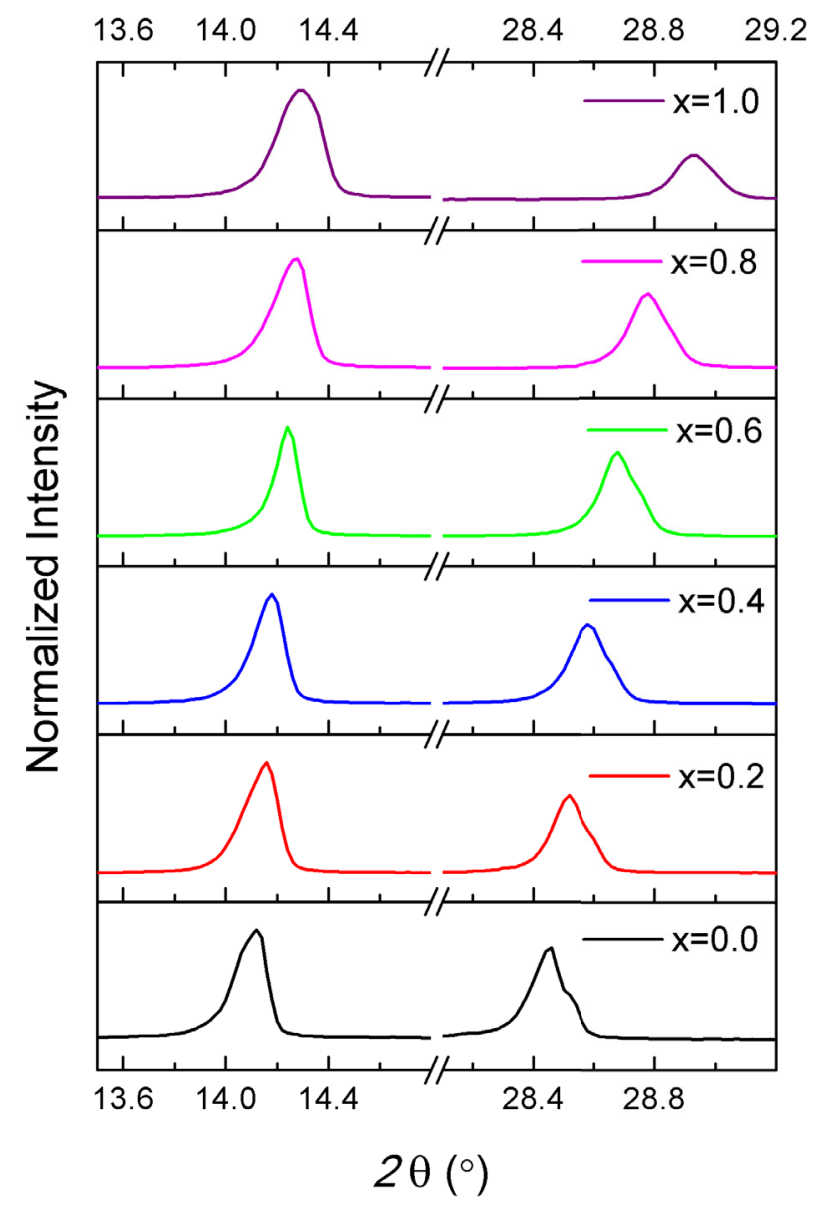

Figure 5.2: $X R D$ of $M A P b I_{3-x} B r_{x}(0 \leq x \leq 1)$ magnified in (100) plane, located between $13.6^{\circ}$ and $14.5^{\circ}$, and (200) plane located between $28.2^{\circ}$ and $30.0^{\circ}$.

Figure 5.2 shows the $X$-ray diffractograms of $M_{A P b I_{3-x}} \operatorname{Br}_{x}(x=0,0.2$, $0.4,0.6,0.8,1.0)$ magnified in the region of the two strongest plane intensities: $13.9^{\circ}-14.5^{\circ}(2 \theta)$ and $28.0^{\circ}-29.4^{\circ}(2 \theta)$. The position of the two peaks shifts to higher angles when the average fraction of bromide introduced in the thin film increases, in agreement with the previous results of Chapter 4. 


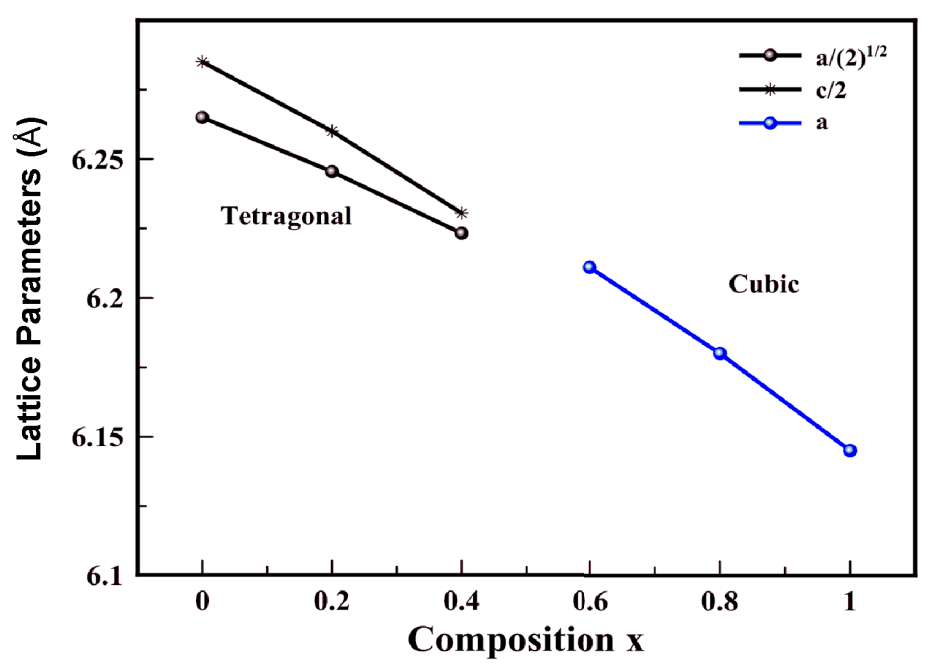

Figure 5.3: Lattice parameters $M A P b I_{3-x} B r_{x}$ as a function of bromide content.

Figure 5.3 reports the evolution of lattice parameters as a function of the bromide content $x$. The calculated lattice parameters were obtained using Lebail fit via Full Prof suite software [229]. The cell parameter $a$ varies quasi-linearly, respecting Vegard's law, with $x$ composition. The parameters $\frac{a}{\sqrt{2}}$ and $\frac{c}{2}$ are the pseudo-cubic parameters calculated from the refined tetragonal cell.

\subsubsection{FESEM and AFM Analyses}

FESEM and AFM analyses were conducted to examine the influence of $B r$ incorporation in the morphology. Figure 5.4 presents top view FESEM images of the $M A P b I_{3-x} B r_{x}$ perovskite thin films deposited onto ITO substrates with different bromide fractions.

The $M A P b I_{3-x} B r_{x}$ film has a denser and homogenous morphology with fiber-like crystals with the presence of voids due to solvent evaporation and some crevices between the crystal boundaries. In the case of $\mathrm{MAPbBr}_{3}$, it 
consists of aggregate crystals with crevices.
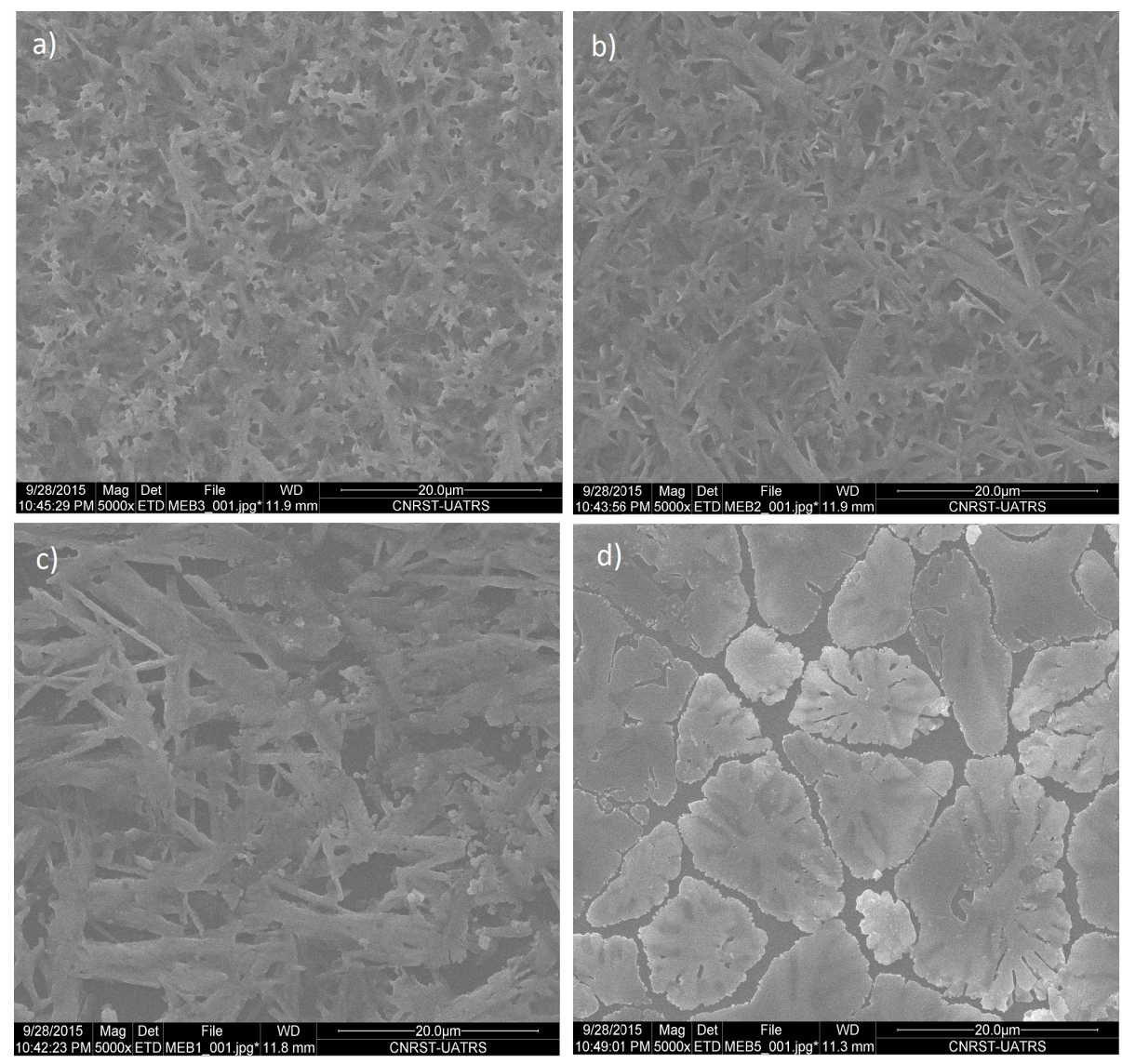

Figure 5.4: FESEM micrographs of thin films with different amount of bromide, (a) $\mathrm{MAPbI}_{3}$, (b) $M A P b I_{2.4} B r_{0.6}$, (c) $M A P b I_{2} B r_{1}$ and (d) $\mathrm{MAPbBr}_{3}$.

Furthermore, FESEM micrographs show the dependence of the grain size on the $B r$ content, $x$ : the obtained films with a different bromide content have a different shape, morphology and size. Indeed, as the composition $x$ increases, the aggregation of grains increased. Figure 5.5 shows AFM images of the $M A P b I_{3-x} B r_{x}$ perovskite thin films with different bromide fractions. 


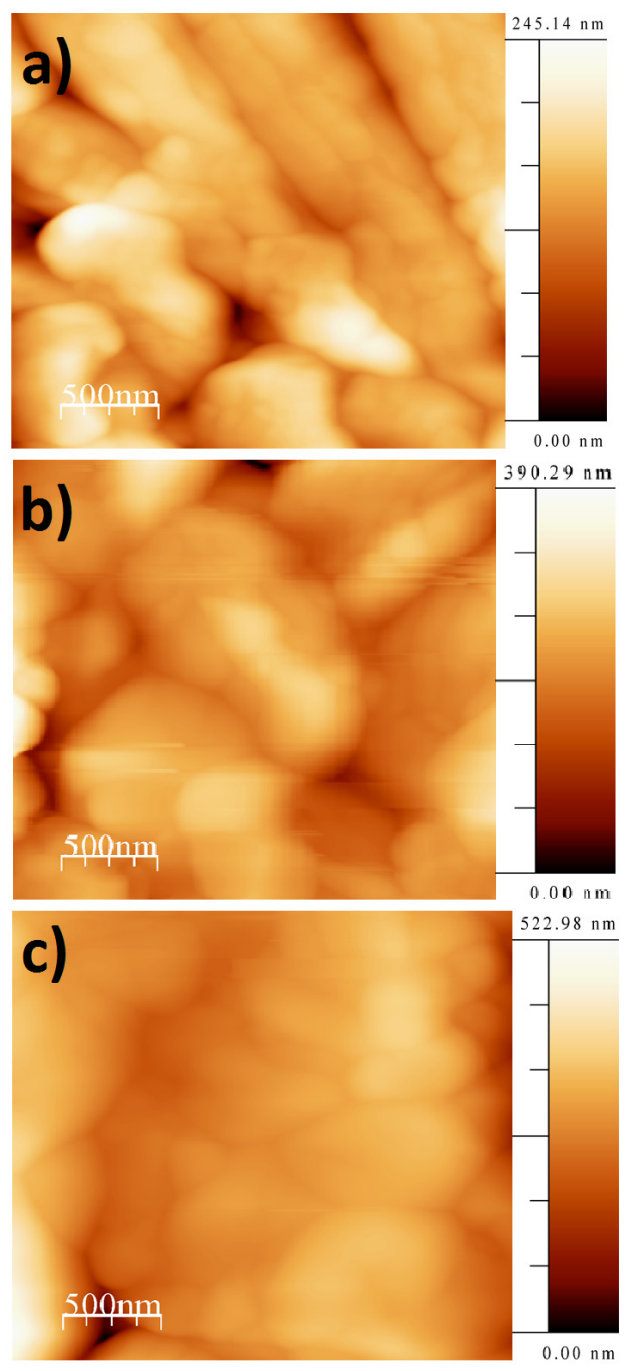

Figure 5.5: AFM images of thin films with different amount of bromide: a) $M A P b I_{2.8} B r_{0.2}$ with $R M S=41.24 \mathrm{~nm}$, b) $M A P b I_{2.4} B r_{0.6}$ with $R M S=$ $42.73 \mathrm{~nm}$ and c) $M A P b I_{2.0} B r_{1.0}$ with $R M S=56.99 \mathrm{~nm}$.

The roughness increases slightly when the bromide fraction increases. The films with a small bromide fraction $(x=0.2)$ have a roughness of 41.24 $\mathrm{nm}$, while it increases to reach $42.73 \mathrm{~nm}$ for $M A P b I_{2.4} B r_{0.6}$ and $56.99 \mathrm{~nm}$ for $\mathrm{MAPbI}_{2.0} B r_{1.0}$. AFM analysis confirms the FESEM micrographs, which showed the formation of aggregate crystals when the $B r$ content increased. 


\subsection{3 $U V$-vis Spectra and Luminescence Properties}

Changing the bromide fraction contained in $\mathrm{MAPbI}_{3-x} \mathrm{Br}_{x}$ affects the optical properties of the perovskite thin films. Figure 5.6 displays the absorbance for $\mathrm{MAPbI}_{3-x} B r_{x}$ for different bromide fractions. As the $\mathrm{Br}$ content increases, the absorbance edge shifts to short wavelength values, indicating the increase of the bandgap energy of the prepared perovskite thin films.

The onset bandgap of mixed bromide iodide perovskite thin films is located in intermediate values between $1.59 \mathrm{eV}\left(\mathrm{MAPbI}_{3}\right)$ and $2.27 \mathrm{eV}\left(\mathrm{MAPbBr}_{3}\right)$, meaning that the bandgap can be tuned by varying the composition of the ratio $I / B r$. This finding is consistent with the works of Sadhanala et al. and Noh et al. who reported that the perovskite bandgap can be tuned by varying the halide $(I / B r)$ composition of the perovskite precursor solution [55, 218]. This increase of the bandgap energy is due to the structural distortion caused by the change in stress of $P b-I$ bonds after $B r$ inclusion [249].

According to absorption measurements, the intensities of the absorbance signal in the $U V$-vis spectra of these films decrease systematically when the fraction of bromide increases. It should be noted that an integrating sphere to collect specular and diffuse transmittance has been used in order to remove the effect of light scattering originating from refraction and reflection phenomena inside the perovskite crystals. However, Tian et al. showed that poor substrate coverage strongly affects the absorption measurement [230].

Figure 5.7 shows the variation of the absorption edge with different halide compositions. The dependence of the absorption edge on the bromide content can be described by the following equation:

$$
E_{g}(x)=E_{g}\left(M A P b I_{3}\right)+\left[E_{g}\left(M A P b B r_{3}\right)-E_{g}\left(M A P b I_{3}\right)-b\right] \frac{x}{3}+b\left(\frac{x}{3}\right)^{2}
$$




$$
E_{g}(x)=1.579+0.0121 x+0.034 x^{2}
$$

where $b$ is the bowing parameter $[231,264]$ reflecting, on the one hand, the fluctuation degree in the crystal field, on the other hand, the nonlinear effect arising from the anisotropic nature of binding [232].

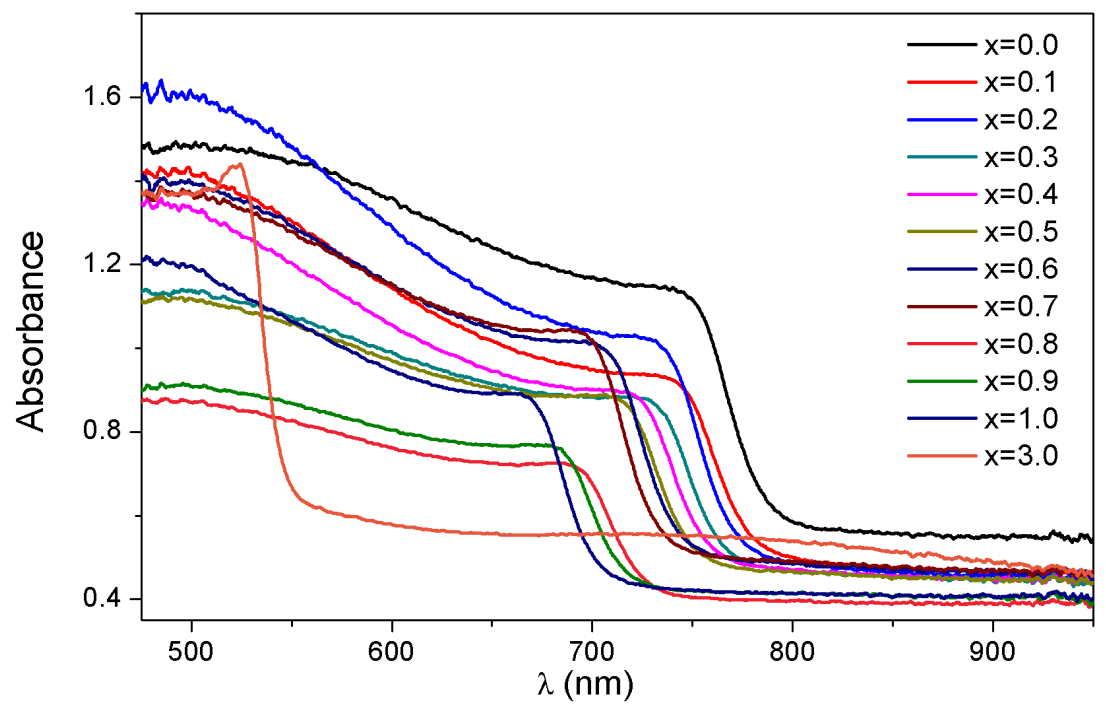

Figure 5.6: $U V$-vis absorption spectra of $M A P b I_{3-x} B r_{x}$ with $0 \leq x \leq 3$.

Fitting equation 5.3.3 to the experimental data shows the nonlinear behavior of $E_{g}$ as a function of $x$, the energies extracted from this data analysis are $E_{g}\left(M A P b I_{3}\right)=1.58 \mathrm{eV}, E_{g}\left(M A P b B r_{3}\right)=2.25 \mathrm{eV}$ and $b=0.30 \mathrm{eV}$. The relatively small $b$ parameter confirms that compositional disorder is low and a great miscibility between $\mathrm{MAPbBr}_{3}$ and $\mathrm{MAPbI}_{3}$. These bandgap values are close to values reported by Noh et al. $\left(E_{g}\left(M A P b I_{3}\right)=1.58 \mathrm{eV}\right.$, $\left.E_{g}\left(\mathrm{MAPbBr}_{3}\right)=2.28 \mathrm{eV}\right)[55]$. The blue shift of the bandgaps might be due to the presence of a $\mathrm{PbI}_{2}$ impurity phase in the prepared samples. 


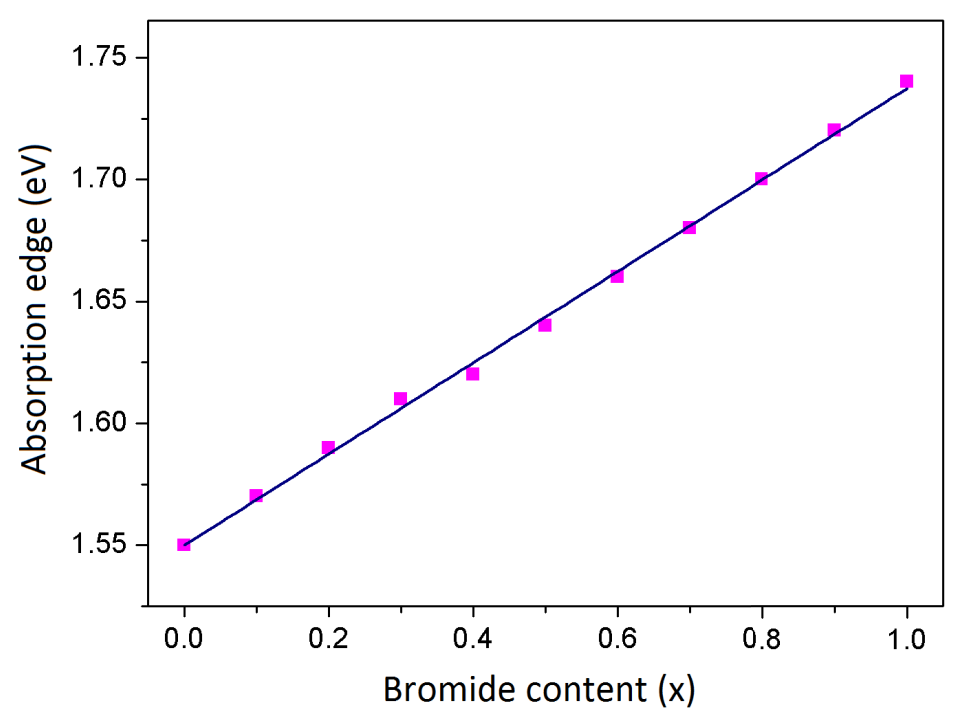

Figure 5.7: Variation of the absorption edge with the chemical composition of bromide for $M A P b I_{3-x} B r_{x}(0 \leq x \leq 1)$.

In Figure 5.8, the $P L$ results of $M A P b I_{3-x} B r_{x}$, formed using the stoichiometric solution with a different fraction of bromide, are compared. Intensities are normalized to clarify the shift of the emission peaks. A systematic shift in the position of the peaks to lower wavelengths is observed as the concentration of bromide increases, which indicates that the recombination centers (defect centers) probably come from the presence of the bromide and iodide in the perovskite lattice, which is in agreement with the $X R D$ analysis. These observations are consistent with the work of Sadhanala et al. [218] suggesting a clean bandgap and one photo-active species from which emission arises in the $\mathrm{Br} / \mathrm{I}$ lead perovskite films. On the other hand, Fedeli et al. [233] reported a quasi-invariant $P L$ energy for intermediate compositions which falls in the $1.67-1.78 \mathrm{eV}$ range, meaning that the emission involves recombination centers that deepen in the bandgap. 


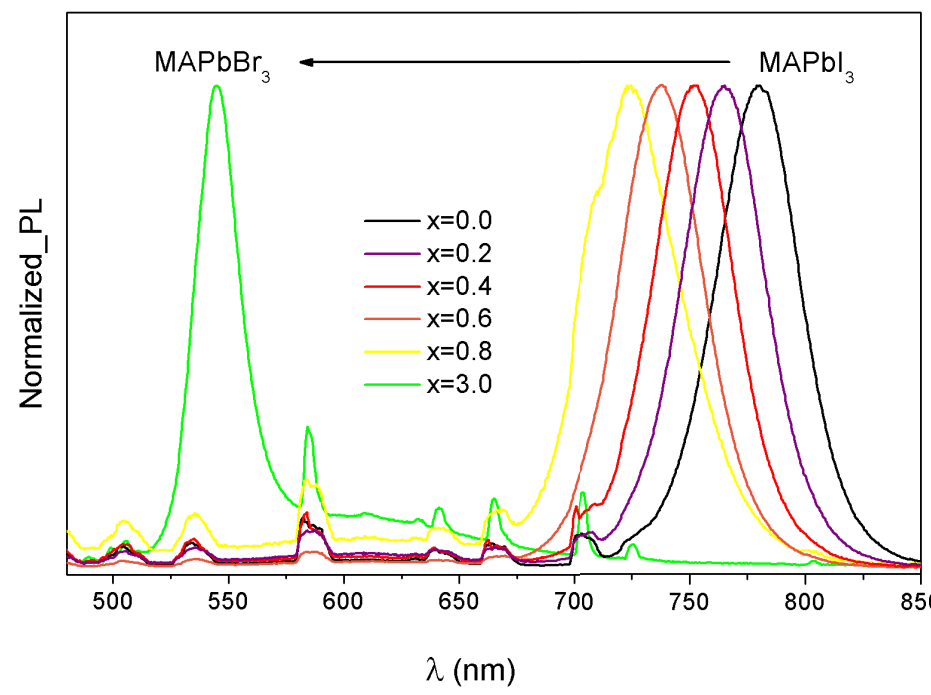

Figure 5.8: $P L$ spectra at room temperature for $M A P b I_{3-x} B r_{x}(0 \leq x \leq 3)$ thin films.

Table 5.1 Position of the absorption edge and the most intense $P L$ peak for $M A P b I_{3-x} B r_{x}$ perovskite thin films recorded at room temperature.

\begin{tabular}{lcccc}
\hline \multirow{2}{*}{ Sample } & Absorption edge & \multicolumn{2}{c}{$P L$ peak position } \\
\hline & \multicolumn{2}{c}{ at $25^{\circ} \mathrm{C}$} & \multicolumn{2}{c}{ at $25^{\circ} \mathrm{C}$} \\
\cline { 2 - 5 }$(\mathrm{nm})$ & $(\mathrm{eV})$ & $(\mathrm{nm})$ & $(\mathrm{eV})$ \\
\hline $\mathrm{MAPbI}_{3}$ & 784 & 1.58 & 781 & 1.58 \\
$\mathrm{MAPbI}_{2.8} \mathrm{Br}_{0.2}$ & 775 & 1.60 & 764 & 1.62 \\
$\mathrm{MAPbI}_{2.6} \mathrm{Br}_{0.4}$ & 761 & 1.63 & 750 & 1.65 \\
$\mathrm{MAPbI}_{2.4} \mathrm{Br}_{0.6}$ & 748 & 1.66 & 738 & 1.68 \\
$\mathrm{MAPbI}_{2.2} \mathrm{Br}_{0.8}$ & 730 & 1.70 & 724 & 1.71 \\
$\mathrm{MAPbBr}_{3}$ & 543 & 2.28 & 545 & 2.27 \\
\hline
\end{tabular}

Table 5.1 shows the energy position of the absorption edge, the position of the most intense $P L$ peak and related wavelength at room temperature 
for different perovskite thin films synthesized in this study. The wavelength position of the maximum of $P L$ emission is shifted to lower wavelengths when compared to the onset of the absorption edge.

\subsection{Conclusion}

Herein, the synthesis of mixed bromide-iodide lead perovskite thin films onto ITO substrate by the spin coating process has been reported.

$X$-ray diffraction analysis indicates the formation of a tetragonal phase $I 4 / m c m$ up to $x=0.4$ and a cubic perovskite with space group $P m \overline{3} m$ in the composition range of $0.6 \leq x \leq 1$. Absorbance measurements show that the perovskite films exhibit a very high absorbance in the visible and short infrared region. As the fraction of bromide changes, the absorption edge of the thin films can be tuned along the visible spectrum from $545 \mathrm{~nm}$ to 789 $n m$.

The bandgap energy of perovskite thin films was estimated from the absorbance spectral measurements, it was found that the onset of the absorption edge for $M A P b I_{3-x} B r_{x}$ thin films ranges from $1.58 \mathrm{eV}\left(\mathrm{MAPbI}_{3}\right)$ to $2.28 \mathrm{eV}\left(\mathrm{MAPbBr}_{3}\right)$. In the $P L$ study, the incorporation of bromide into $M A P b I_{3-x} B r_{x}$ shifts the $P L$ emission to shorter wavelengths, the overall $P L$ emission of mixed bromide-iodide lead perovskite thin films are located in intermediate values between $781 \mathrm{~nm}\left(\mathrm{MAPbI}_{3}\right)$ and $545 \mathrm{~nm}\left(\mathrm{MAPbBr}_{3}\right)$ implying that the defect centers come from the presence of the bromide and iodide in the perovskite lattice. 



\section{Chapter 6}

\section{$M A P b I_{2.9-x} \mathrm{Br}_{x} C l_{0.1}$ Hybrid}

\section{Halide Perovskites: Shedding}

\section{Light on the Effect of}

\section{Chloride and Bromide Ions}

\section{on Structural and}

\section{Photoluminescence Properties}

\subsection{Motivation}

Mixed halide perovskite $M A P b I_{3-x} B r_{x}$ has been studied in detail (see Chapters 4 and 5) and has shown excellent absorption properties with high power conversion efficiency and longer stability than $\mathrm{MAPbI}_{3}$ [234-236]. In addition, $\mathrm{MAPbI}_{3-x} \mathrm{Cl}_{x}$ has been reported to have a longer carrier diffusion length compared with that of $\mathrm{MAPbI}_{3}(\sim 100 \mathrm{~nm})$, and, therefore, an 
improved device performance $[118,240,241]$. Moreover, adding $C l$ to the $\mathrm{MAPbBr}_{3}$ to form $\mathrm{MAPbBr}_{3-x} \mathrm{Cl}_{x}$ increases the open circuit voltage, fill factor, the short circuit current density [55,214], and may reduce the rapid degradation of $\mathrm{MAPbI}_{3}$ in moisture due to the stability of the $\mathrm{MACl}$ structure [242]. Despite the huge number of studies on $\mathrm{Cl} / \mathrm{Br}$ incorporation in mixed halide perovskites, the role of $\mathrm{Cl}$ and $\mathrm{Br}$ in the optoelectronic and morphological characteristics of perovskite films is still of much interest [212, 237, 243-245].

Hence, the aim of this chapter is to gain further insight into the effect of $\mathrm{Cl}$ and $\mathrm{Br}$ addition on the different properties of the $M A P b I_{2.9-x} B r_{x} C l_{0.1}$ perovskite thin films. A study on their structural and optical properties is presented in this section, with the aim to shed light on their properties and optimize their chemical composition, and, thus, meet the requirements of different applications.

\subsection{Experimental Procedure}

The $\mathrm{MAPbI}_{3}, \mathrm{MAPbBr}_{3}$ and $\mathrm{MAPbCl}_{3}$ powders were first synthesized by reacting equimolar ratio of hydrohalide acid $H X(X=I, B r, C l)$ (in water, Sigma Aldrich) with $\mathrm{CH}_{3} \mathrm{NH}_{2}$ (33\% in methanol, Sigma Aldrich) in a round-bottom flask at $0^{\circ} \mathrm{C}$ for $2 h$ with stirring. Then this solution was heated to $100^{\circ} \mathrm{C}$ and $0.030 \mathrm{~mol}$ of $\mathrm{Pb}\left(\mathrm{NO}_{3}\right)_{2}$ previously dissolved in $50 \mathrm{~mL}$ of distilled water) was added dropwise to the solution.

The crystalline perovskite powders precipitate, $\mathrm{MAPbI}_{3}$ with black color and $\mathrm{MAPbBr}_{3}$ with orange color. The remaining solution was then left to cool until around $40^{\circ} \mathrm{C}$ and filtered. The obtained powders were washed with absolute ethanol and diethyl ether several times, then they were dried under vacuum. All materials were purchased from either Sigma-Aldrich or Alfa Aesar 
and used as received.

The precursor solutions were prepared by dissolving $M A P b I_{3}$ and $M A P b B r_{3}$ powders at $45 \% w t$ in dimethylformamide ( $D M F$, Sigma Aldrich) solution and $\mathrm{MAPbCl}_{3}$ at $45 \%$ wt in dimethylsulfoxide (DMSO, Sigma Aldrich). The mixed perovskite solutions were prepared by varying the iodide/bromide/chloride amounts. The mixed solutions were stirred for $3 h$ to ensure the homogeneity of the solution.

Thin film perovskites were then produced by spin coating at a speed of $4000 \mathrm{rpm}$ for $30 \mathrm{~s}$. Finally, the samples were annealed for $1 \mathrm{~h}$ at $100^{\circ} \mathrm{C}$ and kept under vacuum to avoid their degradation.

\subsection{Results and Discussion}

\subsection{1 $X$-ray Analysis}

Figure 6.1 shows the $X$-ray diffractograms for $M A P b I_{3}$ and $M A P b I_{2.9-x} \mathrm{Br}_{x} C l_{0.1}$ with different bromide fractions. The two strongest intensities are centered at about $14.0^{\circ}$ and $28.0^{\circ}$. These peaks are assigned to the crystal planes of the tetragonal lattice of the mixed halide perovskite, indicating that the tetragonal perovskite structure is formed [39]. The peak located at $12.6^{\circ}$ is assigned to $\mathrm{PbI}_{2}$ [120]. No other impurity peaks were detected.

Figure 6.2 shows the $X R D$ patterns of $M A P b I_{2.9-x} B r_{x} C l_{0.1}$, with different bromide fractions, magnified in the region $13.5^{\circ}-14.5^{\circ}(2 \theta)$ and $28.5^{\circ}-30.0^{\circ}(2 \theta)$. As it can be seen, the position of the two peaks shifts to higher angles when the average fraction of bromide and chloride introduced into the thin film increases. This behavior is due to the decrease of the lattice parameter resulting from the difference in the ionic radius of $\mathrm{Cl}^{-}(1.81 \AA)$, $B r^{-}(1.96 \AA)$ and $I^{-}(2.2 \AA)[51]$. Furthermore, $X R D$ analysis indicated the 
absence of a secondary phase, which confirmed the formation of single-phase crystals with a mixed halide lattice.

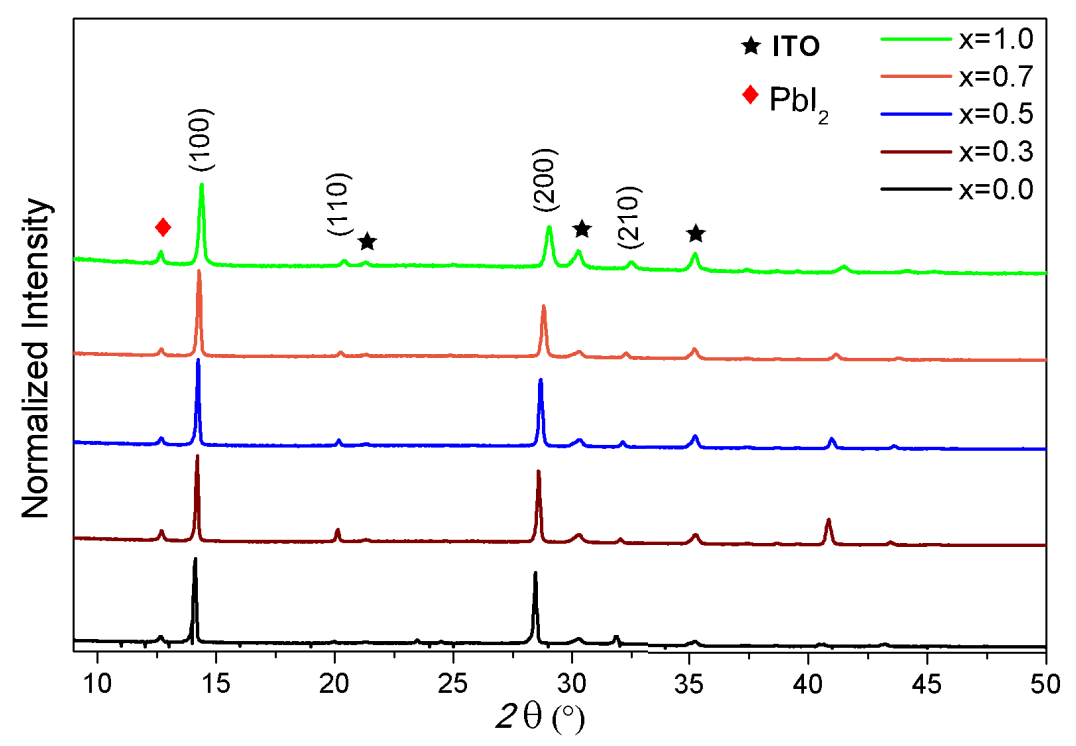

Figure 6.1: $X R D$ patterns of $M A P b I_{3}$ and $M A P b I_{2.9-x} B r_{x} C l_{0.1}$ with $0 \leq$ $x \leq 1$.

In order to gain further insight into the chloride incorporation, the $X R D$ patterns of the $M A P b I_{3-x} B r_{x}(x=0.4,0.8)$ with and without $10 \%$ of chloride were compared (Figures 6.3 and 6.4). It can be observed that the addition of $10 \%$ of chloride produces a slight shift toward higher angles of the peaks (100) and (200), located at $14.2^{\circ}$ and $28.6^{\circ}$ for $\mathrm{MAPbI}_{2.6} \mathrm{Br}_{0.3} \mathrm{Cl}_{0.1}$ and at $14.28^{\circ}$ and $28.8^{\circ}$ for $M A P b I_{2.2} \mathrm{Br}_{0.7} C l_{0.1}$, respectively. This shift might be due to the stresses in the crystal lattice caused by interstitial vacancies, created by the introduction of $10 \%$ of chloride. However, it is unclear whether $\mathrm{Cl}$ occupied an interstitial position in the $M A P b I_{3-x} B r_{x}$ crystal or substitutes $B r$ at the surface of the crystal. 


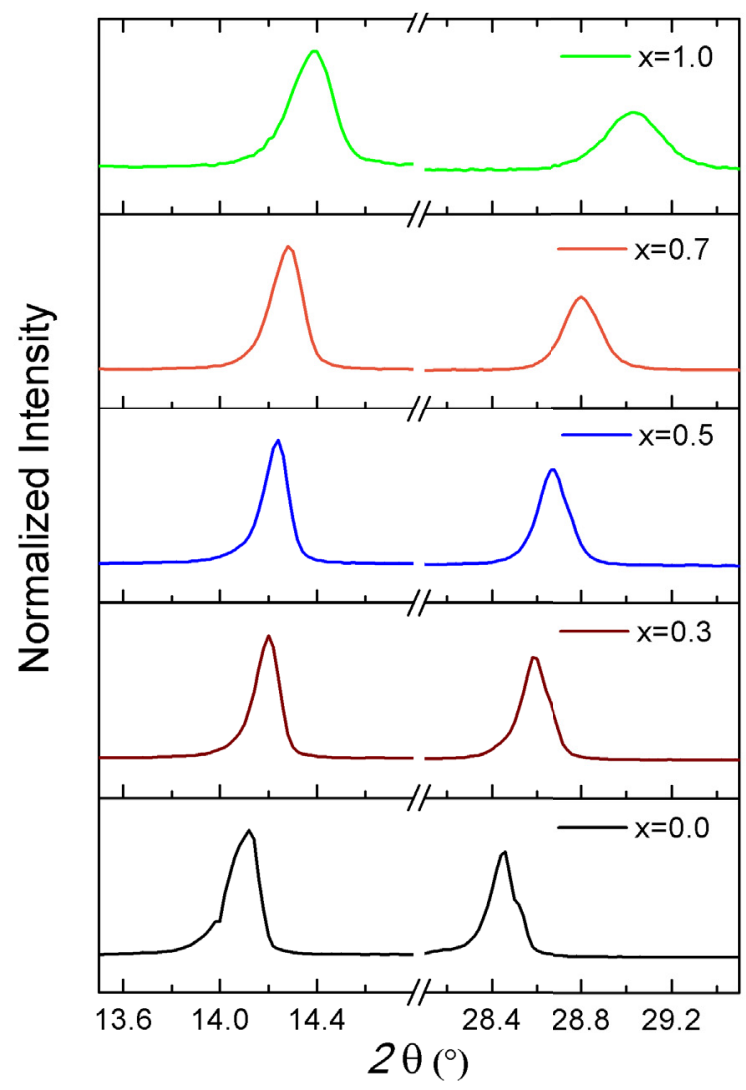

Figure 6.2: $X R D$ patterns of $M A P b I_{2.9-x} B r_{x} C l_{0.1}$, magnified in the region of $13.5^{\circ}-14.5^{\circ}(2 \theta)$ and $28.5^{\circ}-30.0^{\circ}(2 \theta)$.

Some peaks in the cubic-tetragonal region appeared broader, the existence of this double peak with high $F W H M$ values comes from the fact that the planes $(00 l)$ and $(h k 0)$ are related to $c$ and $a=b$ axes [235]. Indeed, in Figure 6.5 the $X R D$ peak at $\sim 28.5^{\circ}$ of the $M A P b I_{2.6} B r_{0.3} C l_{0.1}$ can be decomposed to form two Lorentzian tetragonal peaks, reflections $(004)_{T}(\operatorname{Red} L 1)$ and $(220)_{T}$ (Blue $L 2$ ), explaining the formation of the tetragonal $P 4 / \mathrm{mmm}$ phase up to the open range $0.3 \leq x$ of the bromide content. 

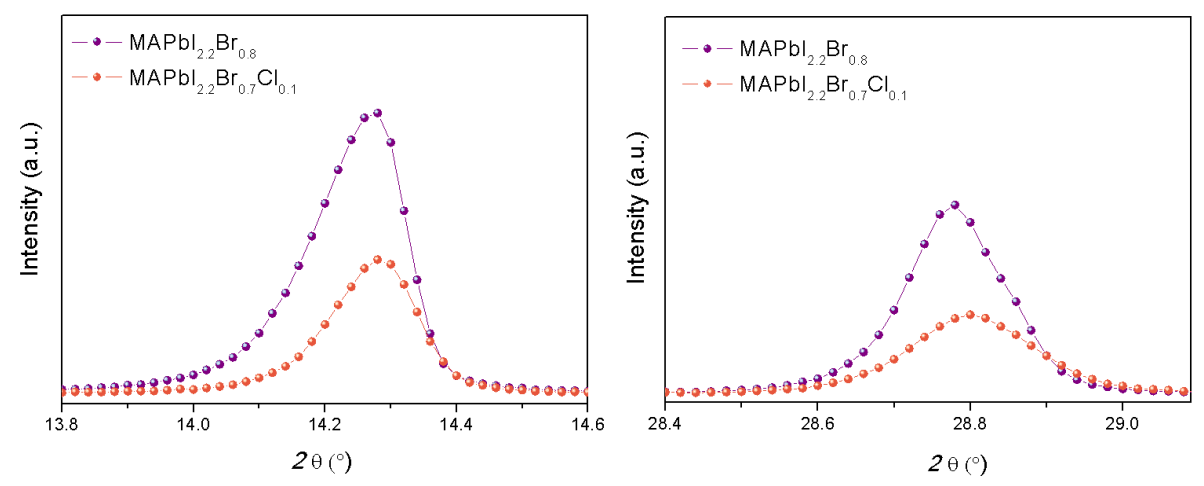

Figure 6.3: $X R D$ of $\mathrm{MAPbI}_{2.2} \mathrm{Br}_{0.8}$ with and without a $10 \%$ of chloride magnified in the region of $2 \theta=13.8^{\circ}-14.6^{\circ}$ and $2 \theta=28.3^{\circ}-29.0^{\circ}$.
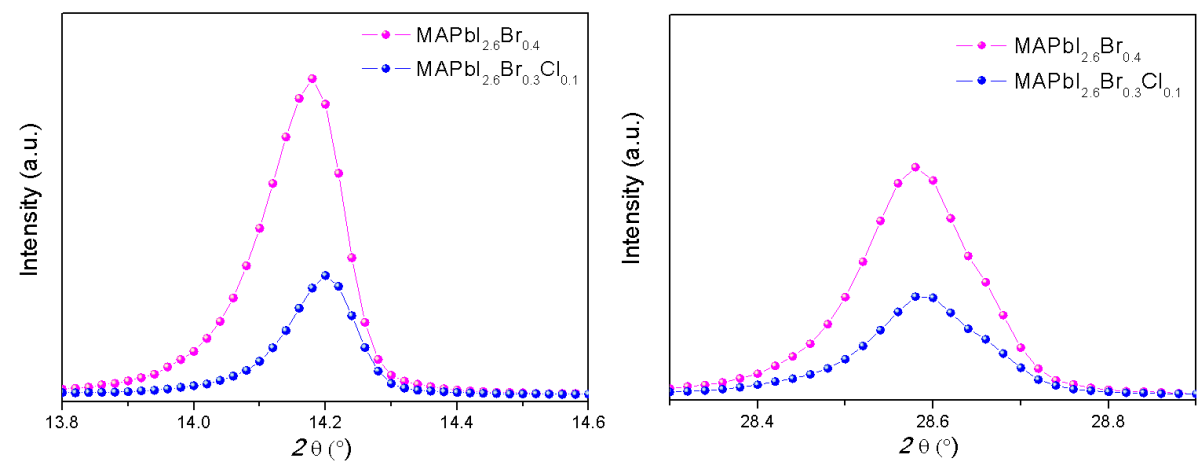

Figure 6.4: $X R D$ of $M A P b I_{2.6} B r_{0.4}$ with and without a $10 \%$ of chloride magnified in the region of $2 \theta=13.8^{\circ}-14.6^{\circ}$ and $2 \theta=28.3^{\circ}-29.0^{\circ}$.

The broadening of the peaks after chloride and bromide inclusion might also indicate a change in the crystallite size (size effect). New defect centers were created in the $\mathrm{MAPbI}_{3}$ host material by doping with chloride and bromide. 


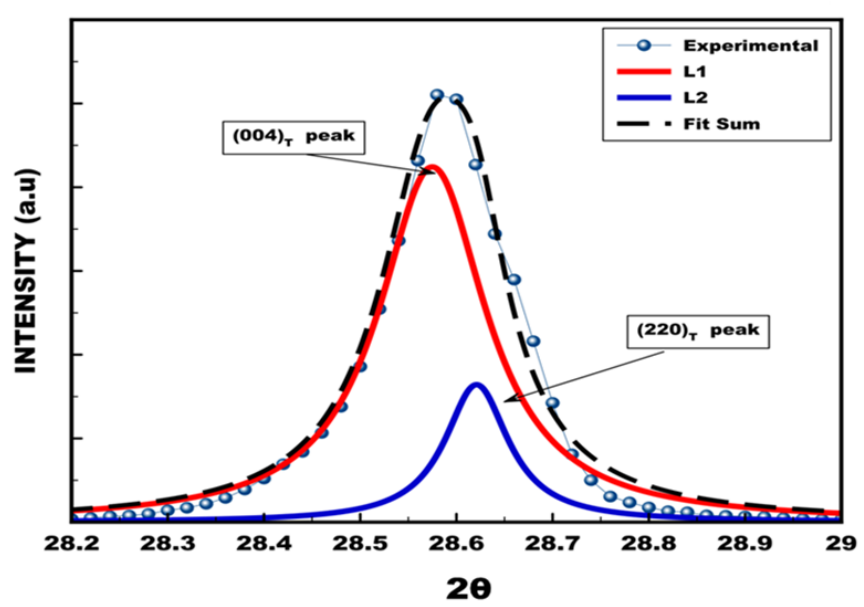

Figure 6.5: Deconvolution of $\mathrm{MAPbI}_{2.6} \mathrm{Br}_{0.3} \mathrm{Cl}_{0.1}$ peak at $28.5^{\circ}$ in two tetragonal peaks.

The Pawley refinement method was performed to fit the peak profiles, indicating the formation of the iodide halide $\mathrm{MAPbI}_{3} \mathrm{Pm}_{\overline{3}} m$ cubic phase for $x=0$ and the formation of the tetragonal $P 4 / m m m$ phase for $x \geq 0.3$.

The cell parameters of the halide thin films were refined using Pawley's fit. The experimental profiles were fitted with the most suitable pseudo-Voigt analytical function, which is represented by the following equation:

$$
p V(x)=\sum I_{\text {int }}[\eta L(x)+(1-\eta) G(x)]
$$

where $L(x)=\left(1+x^{2}\right)^{-1}$ is the Lorentzian component, $G(x)=\exp \left[(\ln 2) x^{2}\right]$ is the Gaussian component for $x=\left(2 \theta-2 \theta_{0}\right) / F W H M$ where $F W H M$ means Full Width at Half Maximum of the Bragg peaks, $\eta$ is the Gaussian character of $X$-ray profiles, $\theta_{0}$ the Bragg angle of $K \alpha_{1}$ peak and Int is the scale factor.

Figures 6.6, 6.7, 6.8 and 6.9 show the Pawley fit of the synthesized samples. ITO peaks were excluded from the diffraction. The factors $R p$ (profile factor) and $R w p$ (weighted profile factor) were used as numerical criteria to evaluate 
the fitting quality of the simulated to experimental data:

$$
\begin{aligned}
R w p & =100 \cdot\left(\frac{\sum w_{i}\left(y_{i}^{\text {obs }}-y_{i}^{\text {calc }}\right)^{2}}{\sum w_{i}\left(y_{i}^{\text {obs }}\right)^{2}}\right)^{\frac{1}{2}} \\
R p & =100 \cdot\left(\frac{\sum\left|y_{i}^{\text {obs }}-y_{i}^{\text {calc }}\right|}{\sum w_{i}\left|y_{i}^{\text {obs }}\right|}\right)
\end{aligned}
$$

where $y_{i}^{o b s}$ is the intensity of experimental profile for a given $2 \theta$ angle, $w_{i}=$ $1 / y_{i}^{o b s}$ is the weight of profile determination and $y_{i}^{\text {calc }}$ is the calculated intensity.

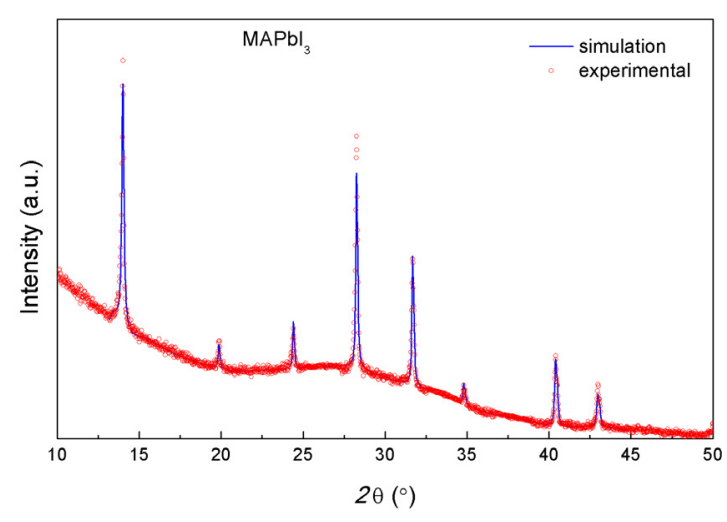

Figure 6.6: Pawley fit of $\mathrm{MAPbI}_{3}$ film.

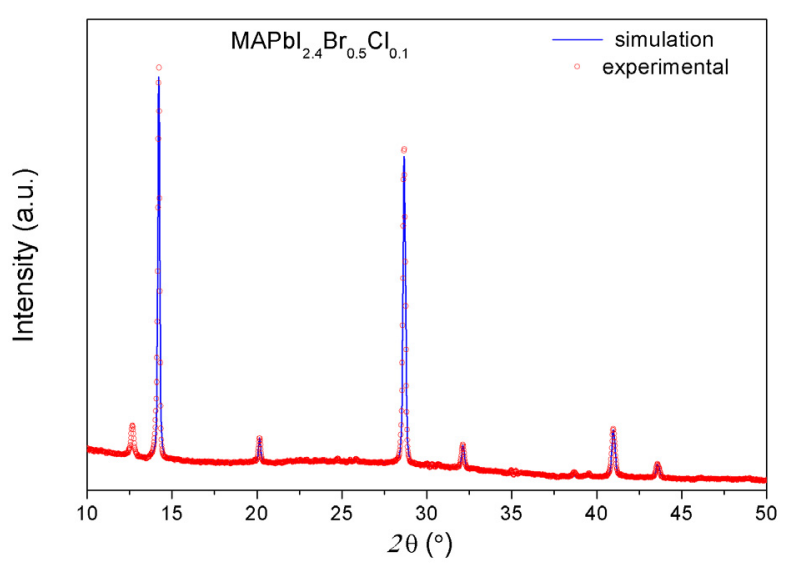

Figure 6.7: Pawley fit of $M A P b I_{2.4} B_{0.5} C l_{0.1}$ film. 


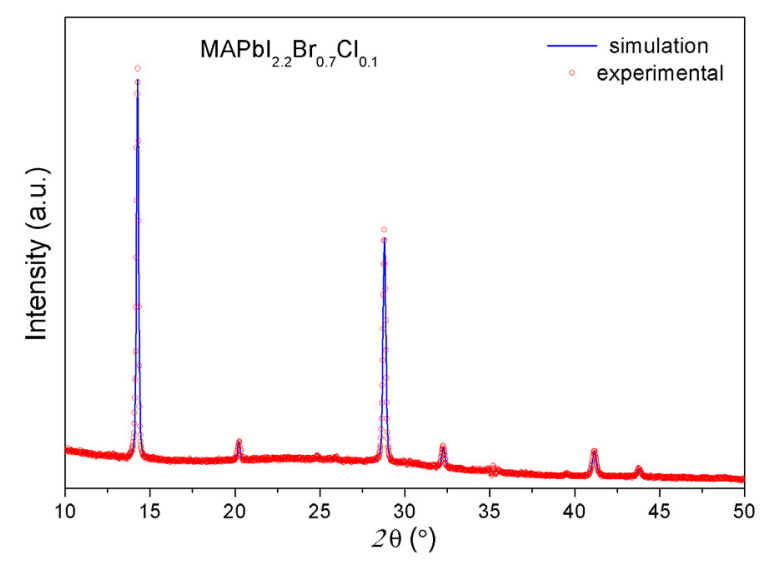

Figure 6.8: Pawley fit of $M A P b I_{2.2} \mathrm{Br}_{0.7} C l_{0.1}$ film.

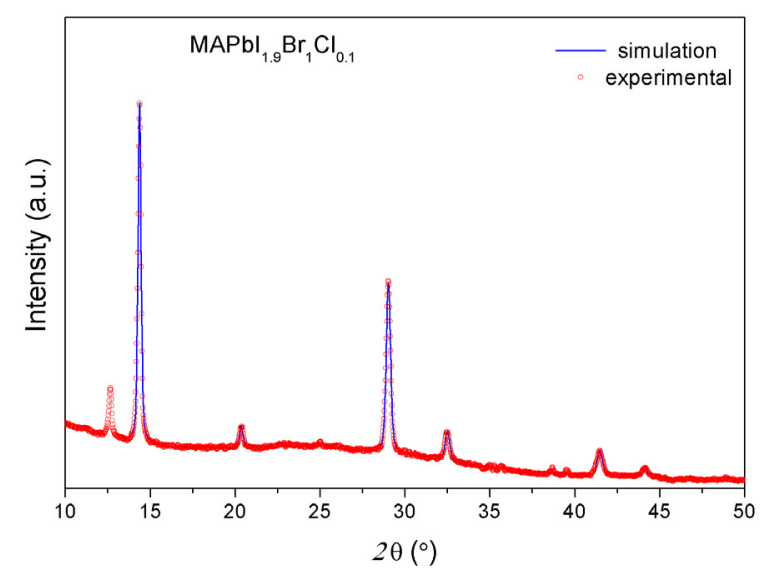

Figure 6.9: Pawley fit of $M A P b I_{1.9} B r_{0.1} C l_{0.1}$ film.

The refined cell parameters through Pawley fit, for the synthesized thin films, are reported in Table 6.1. It is shown that the lattice parameters decrease with the increase of the $\mathrm{Br}$ and $\mathrm{Cl}$ content. 
Table 6.1. Refinement factors and refined cell parameters using the Pawley fit method.

\begin{tabular}{lcccc}
\hline Sample & $a=b(\AA)$ & $c(\AA)$ & $R p$ & $R w p$ \\
\hline$M_{A P b I_{3}}$ & $6.3023(3)$ & $6.3023(3)$ & $2.23 \%$ & $3.23 \%$ \\
$M_{A P b I_{2.6}} \mathrm{Br}_{0.3} C l_{0.1}$ & $4.4189(2)$ & $6.2471(3)$ & $3.43 \%$ & $5.42 \%$ \\
$M A P b I_{2.4} \mathrm{Br}_{0.5} C l_{0.1}$ & $4.4033(2)$ & $6.2247(9)$ & $3.17 \%$ & $4.92 \%$ \\
$\mathrm{MAPbI}_{2.2} \mathrm{Br}_{0.7} C l_{0.1}$ & $4.3814(2)$ & $6.1900(4)$ & $3.03 \%$ & $4.32 \%$ \\
$\mathrm{MAPbI}_{1.9} \mathrm{Br}_{1} \mathrm{Cl}_{0.1}$ & $4.3494(3)$ & $6.1384(5)$ & $1.99 \%$ & $2.81 \%$ \\
\hline
\end{tabular}

\subsubsection{FESEM Analysis}

FESEM analysis was conducted to investigate the film morphology of $M A P b I_{2.9-x} \mathrm{Br}_{x} C l_{0.1}$. Figure 6.10 shows that the perovskite films, with and without chloride, exhibit a similar crystal texture and film uniformity. As can be seen, the films are homogenous and the crystalline particles are aggregated into larger crystals with a larger dimension. In addition, FESEM analysis shows a morphology with irregular coverage and uncovered areas among the perovskite aggregates may have originated from the evaporation of the solvent during film preparation.

It has been found that the presence of $10 \%$ of chloride affects the film morphology since it diminishes the number of voids and uncovered areas. In addition, numerous studies have reported an enhancement of the film reconstruction and mass transport when chloride is incorporated [246, 247]. Furthermore, various studies reported that chloride also slows down the nucleation and growth of perovskite crystals, leading to better quality films $[162,248]$. 


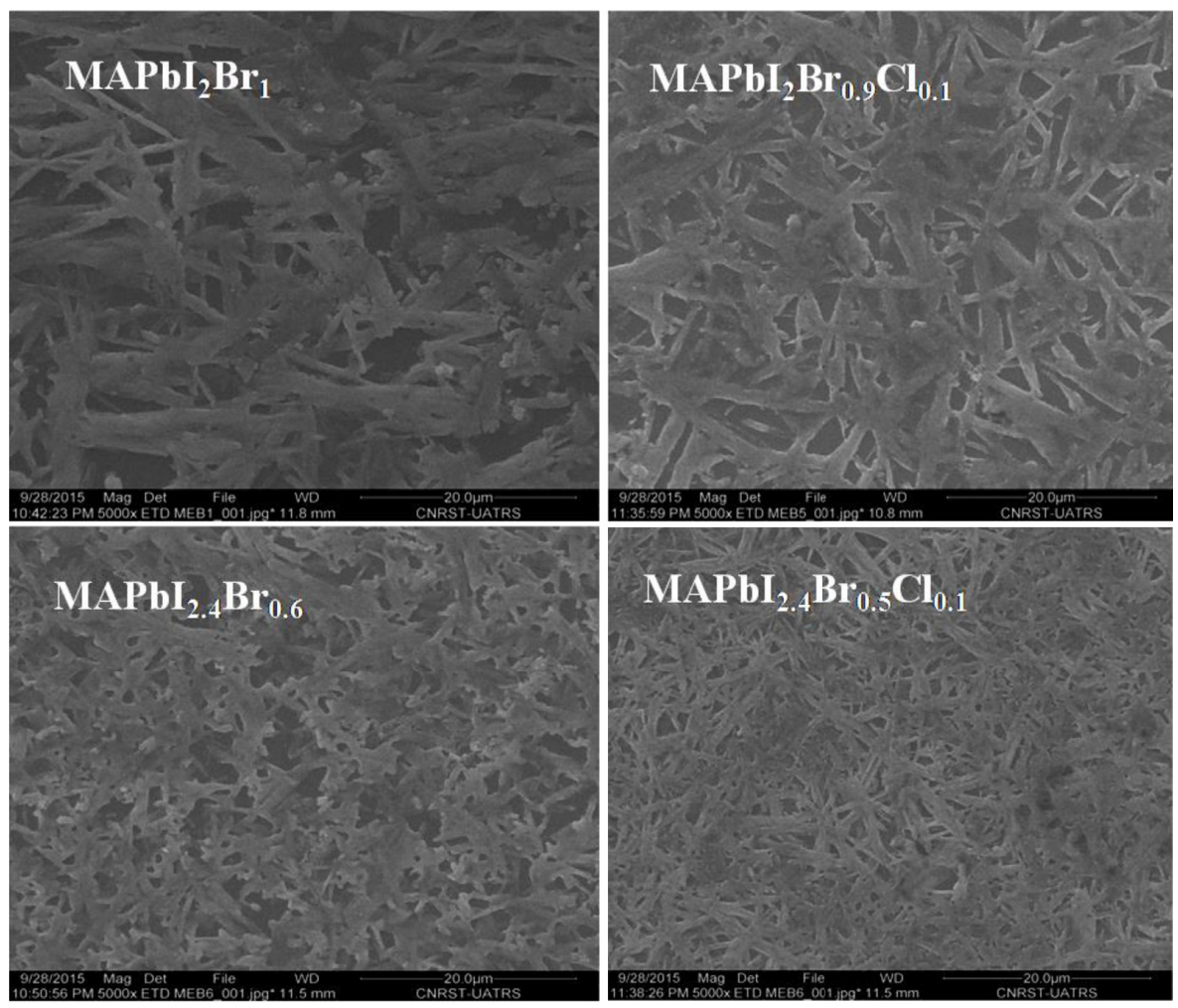

Figure 6.10: Top view FESEM image of $\mathrm{MAPbI}_{2} \mathrm{Br}_{1}, \mathrm{MAPbI}_{2} \mathrm{Br}_{0.9} \mathrm{Cl}_{0.1}$, $\mathrm{MAPbI}_{2.4} \mathrm{Br}_{0.6}$ and $\mathrm{MAPbI}_{2.4} \mathrm{Br}_{0.5} \mathrm{Cl}_{0.1}$.

\subsubsection{Absorbance}

To study the variation of optical properties in thin film perovskites, $U V$-vis absorption spectra measurements of $M A P b I_{2.9-x} \mathrm{Br}_{x} C l_{0.1}(0 \leq x \leq 1)$ perovskites were carried out (Figure 6.11).

The bandgap, estimated from the onset of the absorption edge, increased as the fraction of bromide increased (i.e., perovskite colors change from the brown to the red region), due to the structural distortion caused by the change in stress of $P b-I$ bonds after $B r$ and $C l$ inclusion [249]. Indeed, a bandgap energy value of $1.61 \mathrm{eV}$ was found for $\mathrm{MAPbI}_{2.6} \mathrm{Br}_{0.3} \mathrm{Cl}_{0.1}$ and $1.77 \mathrm{eV}$ for $M A P b I_{1.9} \mathrm{Br}_{1.0} \mathrm{Cl}_{0.1}$. 


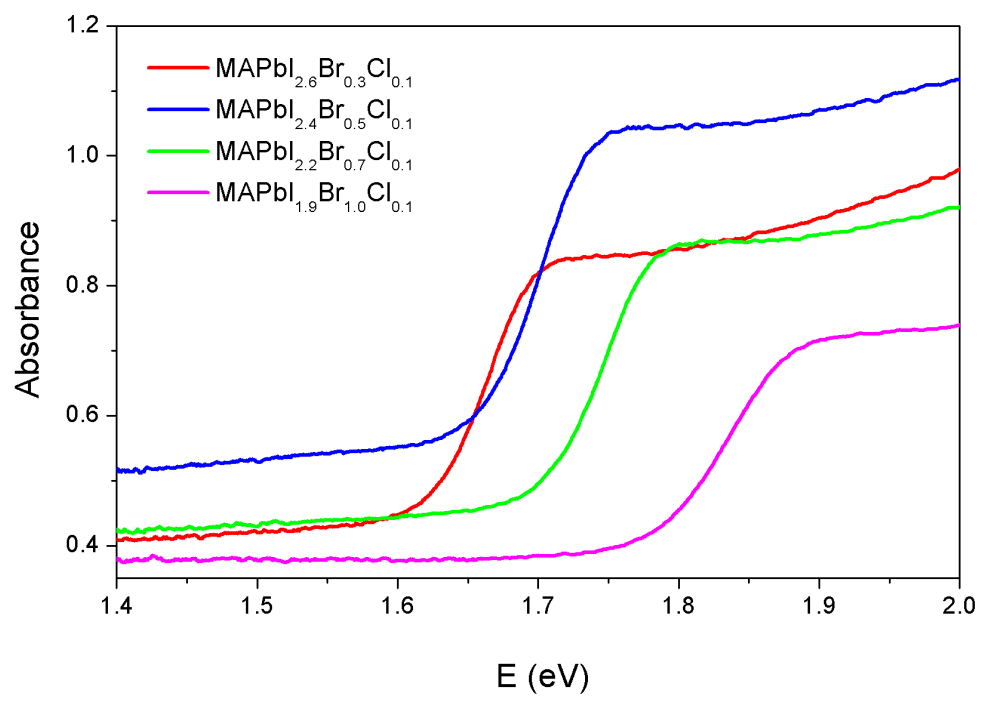

Figure 6.11: $U V$-vis absorption spectra of $M A P b I_{2.9-x} B r_{x} C l_{0.1}$ with $(0 \leq$ $x \leq 1)$.

The effect of chloride incorporation into the $M A P b I_{3-x} B r_{x}$ structure were also studied by measuring the $U V$-vis absorption of mixed hybrid halide perovskites, with and without $10 \%$ of chloride. The results are illustrated in Figure 6.12 and in Table 6.2. These results show that the introduction of a very small fraction of chloride into $M A P b I_{3-x} B r_{x}$ changes the bandgap value slightly, which implies the formation of an increasing amount of vacancies due to the presence of chloride/bromide in the thin films, which would involve additional energy levels in the band gap.

Usually, both bromide and chloride addition shifts the bandgap to higher energy values [240,247,248], although, in this study, the introduction of $10 \%$ of chloride shifts the bandgap slightly toward lower energies, due to the lower quantity of bromide in $\mathrm{MAPbI}_{2.4} \mathrm{Br}_{0.3} \mathrm{Cl}_{0.1}$ compared to $\mathrm{MAPbI}_{2.4} \mathrm{Br}_{0.6}$. 


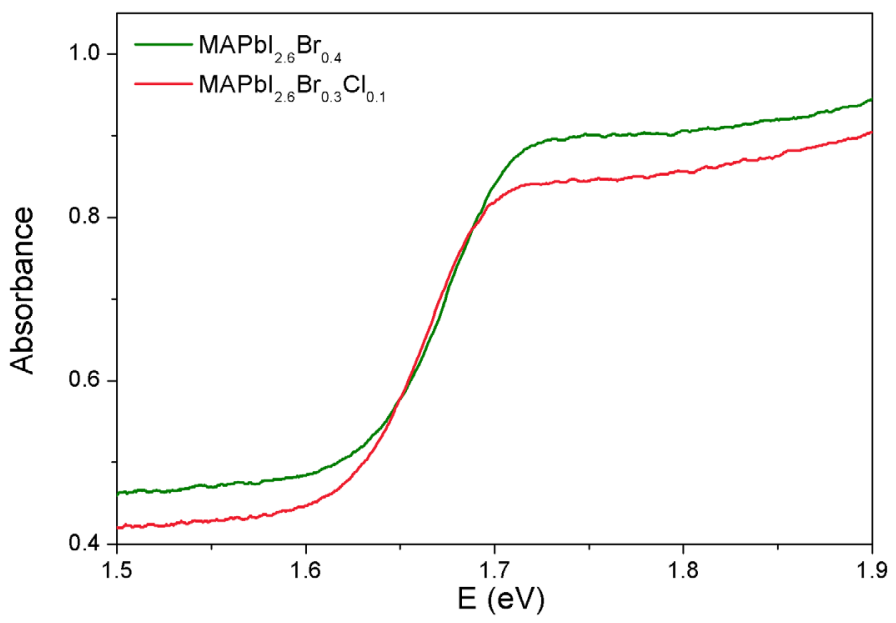

Figure 6.12: $U V$-vis absorption spectra of $M_{A P b I_{2.6}} \mathrm{Br}_{0.4}$ and $\mathrm{MAPbI}_{2.6} \mathrm{Br}_{0.3} \mathrm{Cl}_{0.1}$.

Table 6.2. Bandgap of $M A P I_{3-x} B r_{x}$ compared with $M A P b I_{2.9-x} B r x C l_{0.1}$.

\begin{tabular}{lc}
\hline Sample & Bandgap $(\mathrm{eV})$ \\
\hline $\mathrm{MAPbI}_{2.2} \mathrm{Br}_{0.8}$ & 1.71 \\
$\mathrm{MAPbI}_{2.2} \mathrm{Br}_{0.7} \mathrm{Cl}_{0.1}$ & 1.70 \\
$\mathrm{MAPbI}_{2.4} \mathrm{Br}_{0.6}$ & 1.66 \\
$\mathrm{MAPbI}_{2.4} \mathrm{Br}_{0.5} \mathrm{Cl}_{0.1}$ & 1.64 \\
$\mathrm{MAPbI}_{2.6} \mathrm{Br}_{0.4}$ & 1.63 \\
$\mathrm{MAPbI}_{2.6} \mathrm{Br}_{0.3} \mathrm{Cl}_{0.1}$ & 1.61 \\
\hline
\end{tabular}

\subsubsection{Photoluminescence}

Figure 6.13 displays the photoluminescence measurement recorded on mixed halide perovskite thin films at room temperature. The position of the maximum of the photoluminescence emission is found to shift to a lower 
wavelength as the fraction of bromide increases. This tendency is similar to the one observed in the $U V$-vis absorption analysis. As Gaussian emissions are essentially bromide-dependent, room photoluminescence emission can be tuned from $\sim 730$ to $770 \mathrm{~nm}$ by adding a small fraction of bromide.

In Figure 6.14, it can be observed that $\mathrm{MAPbI}_{2.6} \mathrm{Br}_{0.4}$ presented a higher blue shift than $\mathrm{MAPbI}_{2.6} \mathrm{Br}_{0.3} \mathrm{Cl}_{0.1}$ which showed a slight shift toward higher energies. This shifting tendency was also observed in the $X$-ray patterns of the synthesized films. Therefore, the introduction of $10 \%$ chloride in the halide lattice bandgap level diminishes the blue shifting, meaning that even a small quantity of chloride can affect thin film emissions.

The observed trend in the optical and luminescence spectra is in agreement with the $X$-ray diffraction data of the mixed halide perovskites. Further analyses are needed in order to investigate whether $\mathrm{Cl}$ substitutes $\mathrm{Br}$ or it merely occupied interstitial sites.

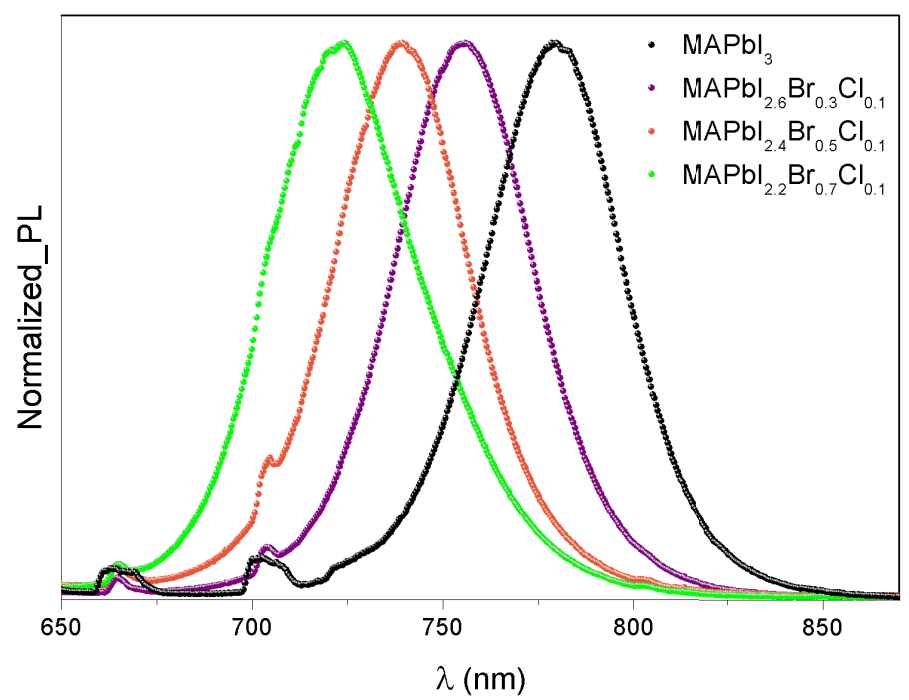

Figure 6.13: $P L$ spectra for $M A P b I_{3}$ and $M A P b I_{2.9-x} B r_{x} C l_{0.1}$ with $0 \leq$ $x \leq 1$. 
The narrower emission bands, with a $F W H M$ of $40 \mathrm{~nm}$, of the perovskite halide thin films are due to the amplified spontaneous emissions [216, 250]. In this study, it has been hypothesized that doping with chloride ions can enhance the near-infrared emissions, and the films can be potential candidates for near-infrared light emitting diodes (NIR-LED). In addition, recently, it has been confirmed that the role of chloride ions can lead to high quality films with exceptional smoothness [251,252].

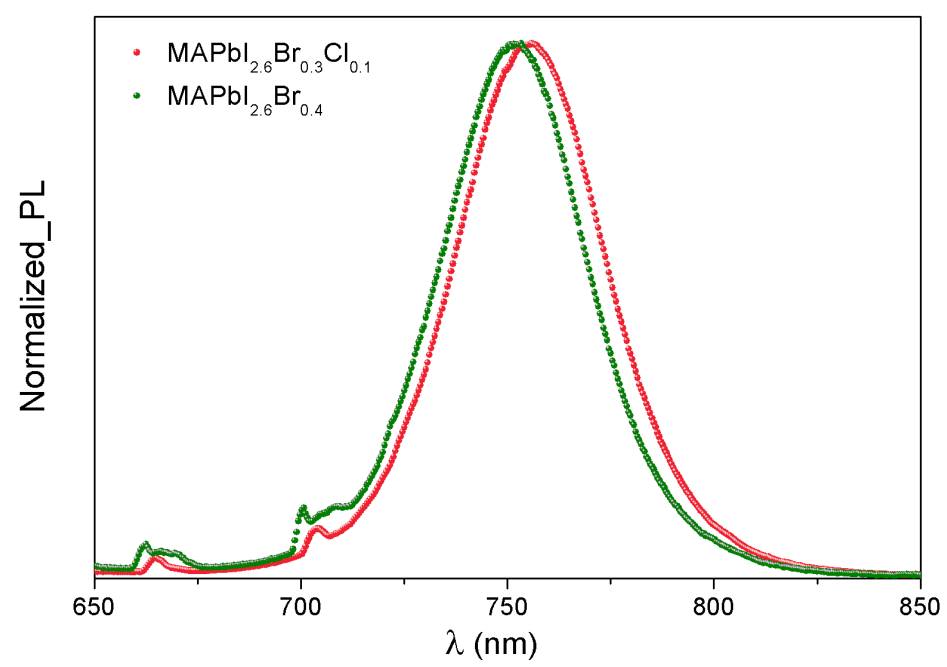

Figure 6.14: Comparison between the $P L$ emissions of $M A P b I_{2.6} B r_{0.4}$ and $\mathrm{MAPbI}_{2.6} \mathrm{Br}_{0.3} \mathrm{Cl}_{0.1}$.

We believe that the effect of chloride ions on the $P L$ emission of $M A P b I_{2.9-x} \mathrm{Br}_{x} C l_{0.1}$ is similar to its effect on $M A P b B r_{3-x} C l_{x}$ and $\mathrm{MAPbI}_{3-x} \mathrm{Cl}_{x}$ thin films. Yet, other parameters should be carefully taken into account when studying the effect of bromide and chloride incorporation in mixed lead halide perovskite films. Weil et al. reported that the annealing temperature of perovskite $M A P b B r_{3-x} C l_{x}$ can lead to the decomposition of $M A C l$ during the annealing step, inducing the blue shift of the emission 
peak [253]. In addition, Yan et al. revealed that not only the $C l$ affects the photoluminescence properties of the $\mathrm{MAPbBr}_{3-x} C l_{x}$ perovskite films, but also the $M A B r$ concentration [245].

\subsection{Conclusion}

In this chapter, the structural and optical properties of perovskite thin films, as well as the effects of $\mathrm{Cl}$ and $\mathrm{Br}$ incorporation on different properties of $\mathrm{MAPbI}_{2.9-x} \mathrm{Br}_{x} \mathrm{Cl}_{0.1}$, have been investigated. The films, deposited by the spin coating process, were homogeneous and adherent to the substrate with a strong absorbance in the $U V$-vis range. The Pawley fit method indicates the formation of the iodide halide $\mathrm{MAPbI}_{3} \mathrm{Pm} \overline{3} \mathrm{~m}$ cubic phase for $x=0$ and tetragonal $P 4 / \mathrm{mmm}$ phase for $x \geq 0.3$. In addition, the onset of the absorption edge for $M A P b I_{2.9-x} B r_{x} C l_{0.1}$ thin film perovskites ranges from 1.60 to $1.80 \mathrm{eV}$ when $0.3 \leq x \leq 1$. Moreover, it was found that both $\mathrm{Cl}$ and $\mathrm{Br}$ affect the $P L$ emission of the mixed halide lead perovskite, the $\mathrm{MAPbI}_{2.9-x} \mathrm{Br}_{x} C l_{0.1}$ films displayed intermediate values from $730 \mathrm{~nm}$ $\left(\mathrm{MAPbI}_{2.2} \mathrm{Br}_{0.7} \mathrm{Cl}_{0.1}\right)$ to $770 \mathrm{~nm}\left(\mathrm{MAPbI}_{2.6} \mathrm{Br}_{0.3} \mathrm{Cl}_{0.1}\right)$. 


\title{
Chapter 7
}

\section{Impact of Iodide Substitution}

\author{
on the Physical Properties
}

\section{and Stability of Cesium Lead}

\section{Halide Perovskite Thin Films}

$\mathrm{CsPbBr}_{3-x} I_{x}(0 \leq x \leq 1)$

\subsection{Motivation}

As mentioned in the introduction, methylammonuim $(M A)$ and formamidinium $(F A)$ perovskite materials [52,108, 118, 123,235, 250, 255-257], have shown outstanding optoelectronic properties, although the presence of the organic cation in these compounds may reduce their stability [223]. Indeed, the decomposition of $\mathrm{MAPbI}_{3}$ in the presence of molecular oxygen, light or under ambient conditions has been widely reported [74,258-260]. With the aim of improving stability, inorganic cesium lead halide $C s P b X_{3}$ perovskite 
has been studied as an alternative for $M A P b X_{3}$ or $F A P b X_{3}(X=I, B r, C l)$. However, the results from this research have shown some drawbacks. For example, it has been shown that the cubic phase of $\mathrm{CsPbI}_{3}$ forms only at temperature higher than $300^{\circ} \mathrm{C}$ and degrades rapidly in ambient air to a yellow non-perovskite phase, although $\mathrm{CsPbBr}_{3}$ has been reported to be a very stable perovskite material with high electron mobility $\left(2300 \mathrm{~cm}^{2} \mathrm{~V} \mathrm{~s}^{-1}\right)$, high hole mobility $\left(320 \mathrm{~cm}^{2} V \mathrm{~s}^{-1}\right)$ and an estimated electron lifetime of $2.5 \mathrm{~ms}[40,261]$. These latter results have recently encouraged new far-reaching research on the use of $C s$ containing perovskite in solar cells and, as a result, Kulbak et al. have reported a $C s \mathrm{PbBr}_{3}$ solar cell with a $P C E$ of about $5 \%$ [262] and Sutton et al. have reported a $\mathrm{CsPbIBr} r_{2}$ based device with a PCE higher than 9.8\% [263]. However, the relatively large bandgap of the $\mathrm{CsPbr}_{3}$ films and the fast degradation of $\mathrm{CsPbI}$ are limiting their use as an active layer in photovoltaic devices. Therefore, in this research mixed cesium lead halide $C s \mathrm{PbBr}_{3-x} I_{x}$ perovskite thin films have been synthesized, using the spin coating process, in order to gain further insight into the effect of iodide substitution on the physical properties and the stability of $\mathrm{CsPbBr}_{3-x} I_{x}$ perovskite material.

\subsection{Experimental section}

Indium tin oxide (ITO) substrates were cleaned by ultrasonication in $2 \%$ Helmanex soap in de-ionized water, isopropyl alcohol and then acetone for 5 min each. After solvent cleaning, the substrates were dried with $A r$ blow. $C s \mathrm{PbBr}_{3-x} I_{x}$ films were prepared by mixing a stoichiometric ratio of $C s X$ and $\mathrm{PbX}_{2}(X=I, \mathrm{Br}, 99.999 \%$, Sigma Aldrich). The precursors were dissolved at $48 \%$ wt in $D M S O(\geq 99.9 \%$, Sigma Aldrich) under an inert atmosphere in an Argon glove box. The solutions were kept under magnetic stirring at $60^{\circ} \mathrm{C}$ 
for $24 \mathrm{~h}$. The films were deposited, inside the glove box, using the spin coating process at $5000 \mathrm{rpm}$ for $30 \mathrm{~s}$, then annealed at $90^{\circ} \mathrm{C}$ for $30 \mathrm{~min}$. Finally, the samples were kept in the glove box before analysis.

\subsection{Results and Discussion}

\subsubsection{X-ray Analysis}

$X$-ray analysis of $C s \mathrm{PbBr}_{3-x} I_{x}$ samples shown in Figure 7.1 indicated that $\mathrm{CsPbBr} r_{3}$ crystallizes in the orthorhombic (Pnma) space group structure, showing several characteristic diffraction profiles, specifically the peaks at $15.21^{\circ}, 21.6^{\circ}$ and $30.6^{\circ}$ associated with the planes (101), (121) and (202), respectively. The intensity of (202) diffraction peak is significantly high compared to bibliographic data, indicating that the perovskite films are oriented in the (202) direction.

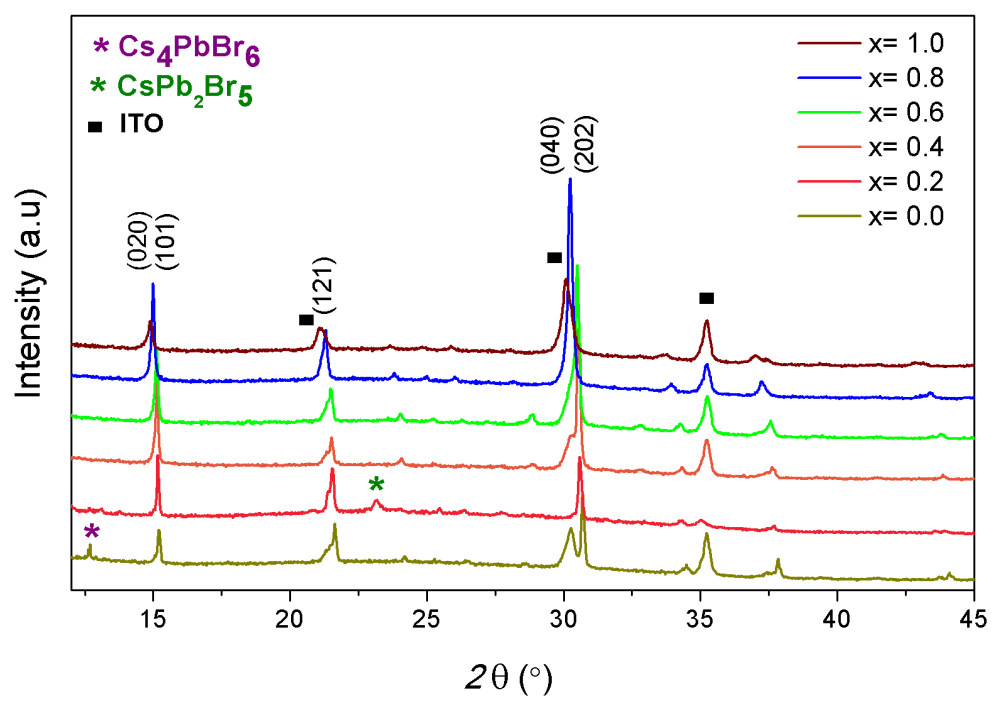

Figure 7.1: $X R D$ of $C s P b B r_{3-x} I_{x}(0 \leq x \leq 1)$ thin films. 
The lattice parameters obtained were $a=8.25(7) \AA, b=11.70(1) \AA$ and $c=8.21(1) \AA$. Thus, the measured spectrum for $\mathrm{CsPbBr}_{3}$ is in a good agreement with the orthorhombic perovskite structure and lattice parameters from data in the literature $(a=8.24(3) \AA, b=11.73(5) \AA$, and $c=8.19(8) \AA)$ [261].

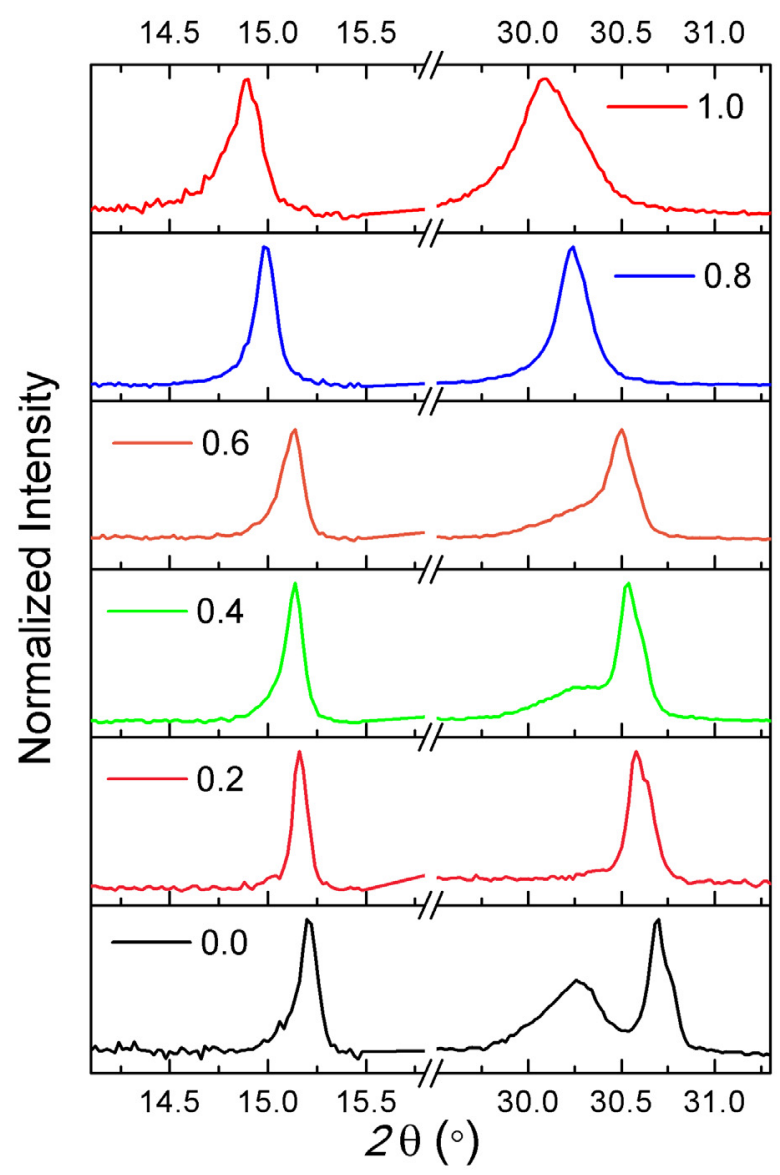

Figure 7.2: $X$-ray diffractograms of $C s P b B r_{3-x} I_{x}(0 \leq x \leq 1)$ magnified in the region of the strongest plan intensity: (100) plan located between $14.5^{\circ}$ and $15.5^{\circ}(2 \theta)$, and the (200) plan located between $30.5^{\circ}-31^{\circ}(2 \theta)$. 
Diffraction peaks located at $12.88^{\circ}$ and $23.15^{\circ}$ indicated the presence of $\mathrm{Cs}_{2} \mathrm{PbBr}_{4}$ and $\mathrm{CsPb}_{2} \mathrm{Br}_{5}$ crystallites respectively, possibly due to the weak solubility of $\mathrm{CsBr}$ and $\mathrm{PbBr}_{2}$ in the $\mathrm{DMSO}$ solvent.

The $C s \mathrm{PbBr}_{3-x} I_{x}$ spin coated films gradually changed their colors in a few seconds of the thermal annealing process, approximately at $90^{\circ} \mathrm{C}$ to evaporate the organic solvent, indicating the relatively fast crystallization of cesium lead perovskite films.

Figure 7.2 shows the $X$-ray diffractograms of $C s P b B r_{3-x} I_{x}$ samples magnified in the region of the two strongest plane intensities: $14.5^{\circ}-16.0^{\circ}$ $(2 \theta)$ and $28.0^{\circ}-31.0^{\circ}(2 \theta)$. As can be seen, the position of the two peaks shifts to lower angles due to the lattice expansion when bromide (ionic radius $1.96 \AA$ ) is substituted for iodide (ionic radius $2.2 \AA$ ).

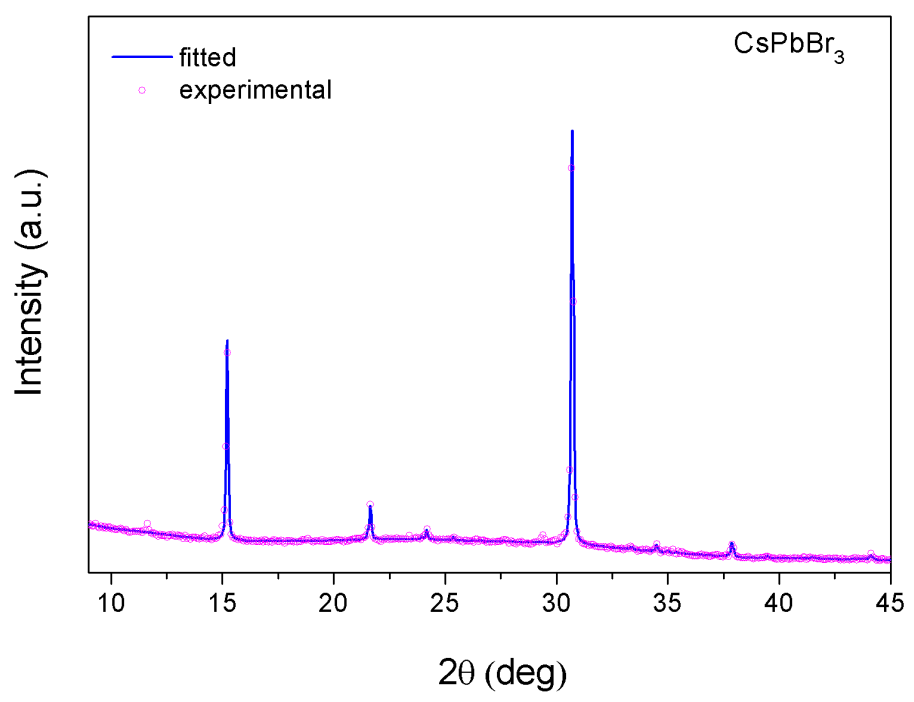

Figure 7.3: Pawley fit of the $\mathrm{CsPbBr}$ thin film. 


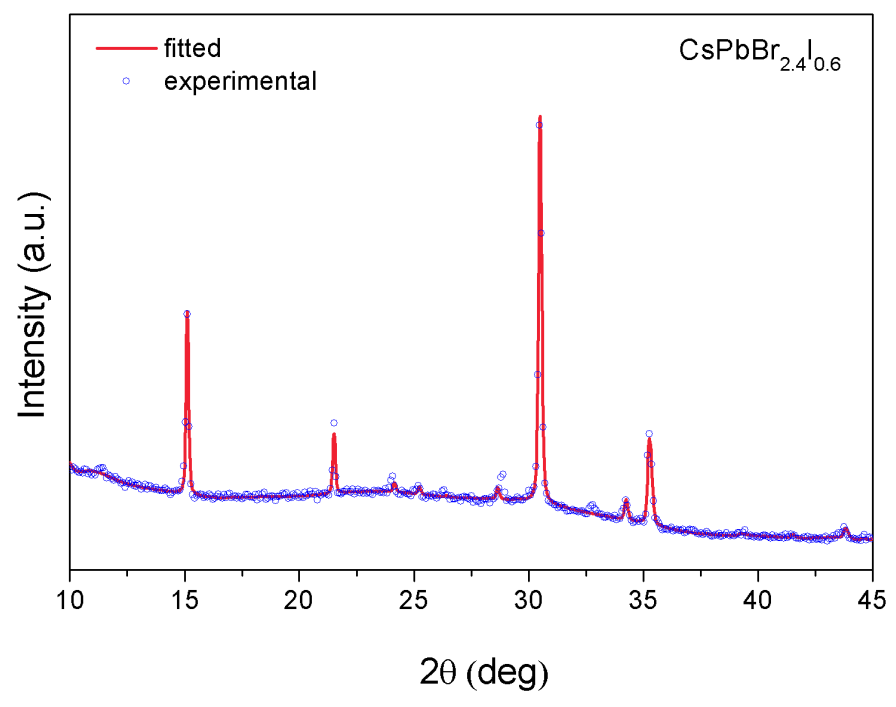

Figure 7.4: Pawley fit of $C s \mathrm{PbBr}_{2.4} I_{0.6}$ thin film.

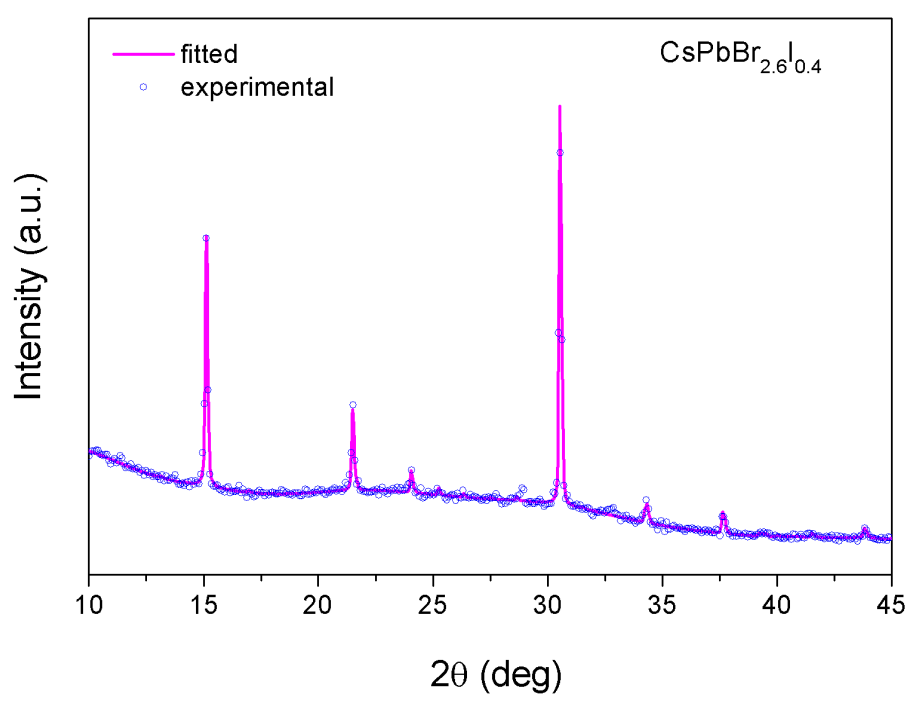

Figure 7.5: Pawley fit of the $C s P b B r_{2.6} I_{0.4}$ thin film. 


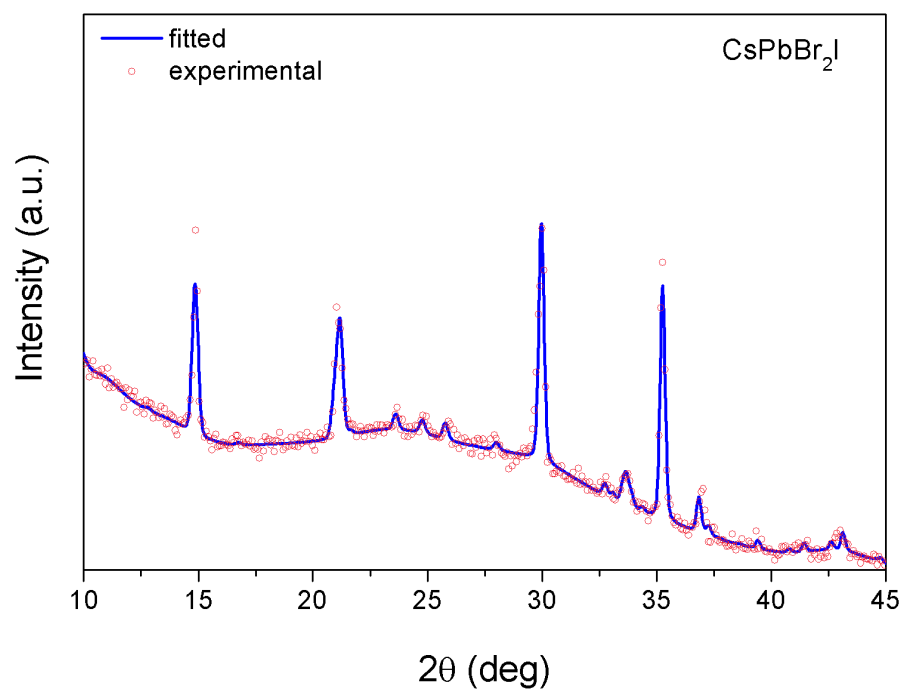

Figure 7.6: Pawley fit of the $\mathrm{CsPbBr} r_{2} I$ thin film.

The Pawley refinement method was performed to fit the peak profiles, indicating that the mixed lead halide $C s \mathrm{PbBr}_{3-x} I_{x}$ perovskite crystallizes in the orthorhombic (Pnma) space group. Figures 7.3, 7.4, 7.5 and 7.6 show the Pawley fit of synthesized mixed cesium lead halide perovskite thin films $C s \mathrm{PbBr}_{3-x} I_{x}$. The cell parameters of the $C s \mathrm{PbBr}_{3-x} I_{x}$ lattice were refined through Pawley fit and are shown in Table 7.1. The refined cell parameters for the synthesized thin films are reported in Figure 7.7, confirming the lattice expansion with the increase of the iodide content.

The lattice parameters of $\mathrm{CsPbBr}_{3}$ calculated in this research match well with the orthorhombic (Pnma) crystal structure reported in [261], with the lattice parameters of $a=8.26(3) \AA, b=11.79(5) \AA$ and $c=8.19(3) \AA$. 
Table 7.1 Refined cell parameters using the Pawley fit method.

\begin{tabular}{llllll}
\hline Sample & Group & $a(\AA)$ & $b(\AA)$ & $c(\AA)$ & $R w p$ \\
\hline$C s P b B r_{3}[41]$ & Pnma & 8.24400 & 11.7351 & 8.19820 & \\
$C s P b B r_{3}$ & Pnma & 8.25744 & 11.70180 & 8.21118 & $4.24 \%$ \\
$C_{s P b B r} I_{2.6} I_{0.4}$ & Pnma & 8.27629 & 11.69357 & 8.20048 & $3.78 \%$ \\
$C s P b B r_{2.4} I_{0.6}$ & Pnma & 8.24781 & 11.72234 & 8.21707 & $4.34 \%$ \\
$C s P b B r_{2.0} I_{1.0}$ & Pnma & 8.21092 & 11.92314 & 8.48119 & $4.34 \%$ \\
\hline
\end{tabular}

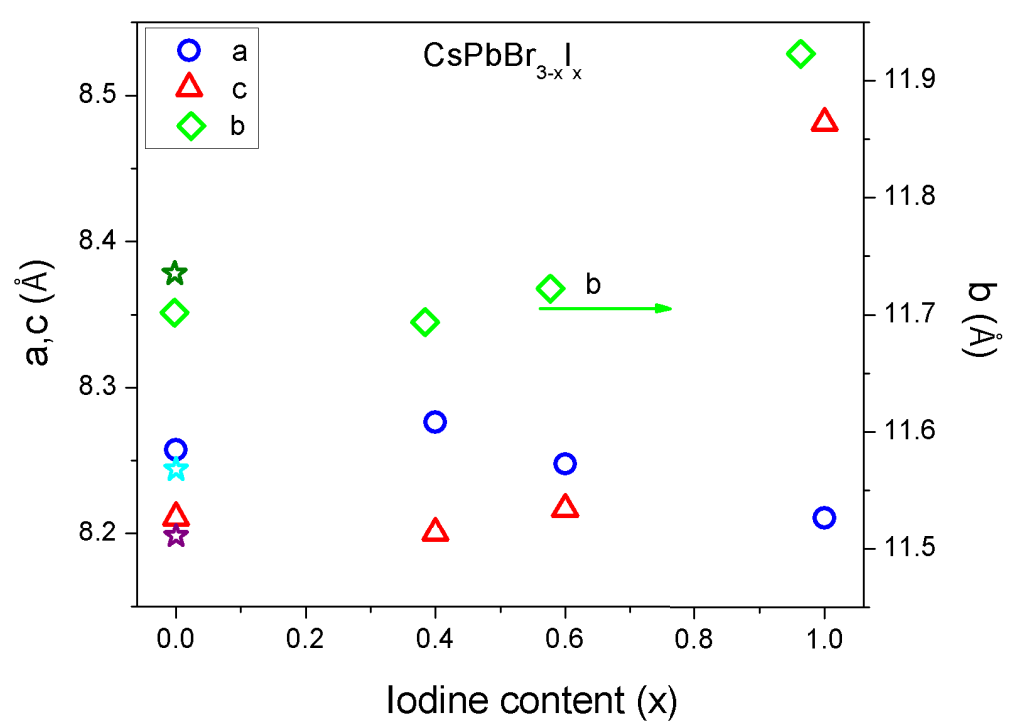

Figure 7.7: Dependence on $x$ of the lattice parameters $a, b$ and $c$ of $\mathrm{CsPbBr} r_{3-x} I_{x}$ orthorhombic. The stars represent data of $\mathrm{CsPbBr}$ reported by Stoumpos et al. [261].

Also, the dependence of the full-width at half-maximum $(F W H M)$ on the $X R D$ peak (200) was investigated. Figure 7.8, shows how FWHM values increase when the iodide fraction increases, which may be due to a variation with $x$ of the grain size, according to the Scherrer equation. Therefore, iodide 
substitution increases the grain size of the $C s P b B r_{3-x} I_{x}$ perovskite.

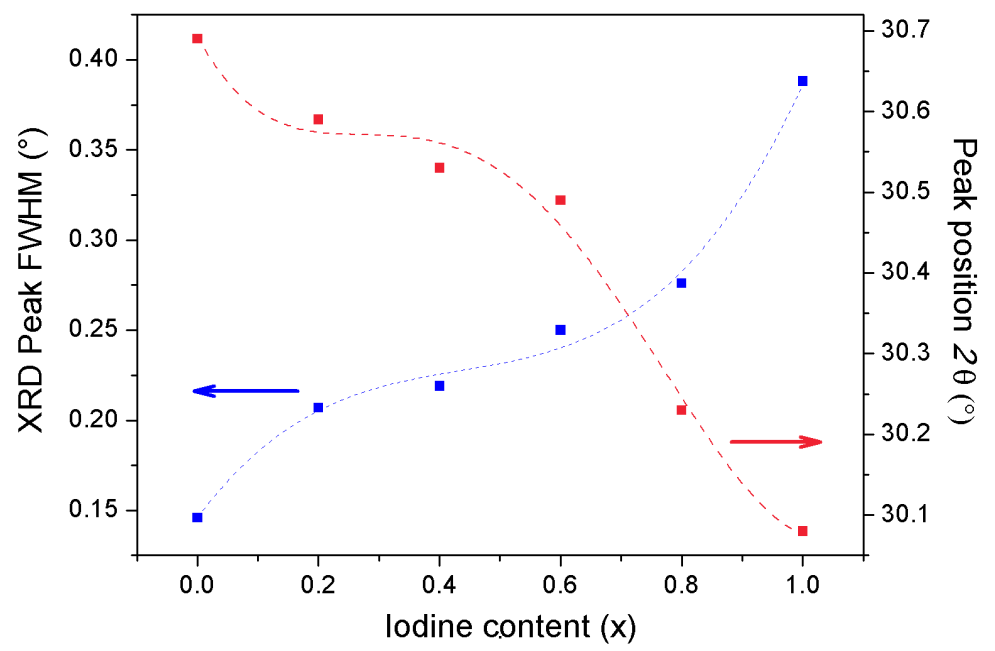

Figure 7.8: Dependence on $x$ of the $F W H M$ of the $X R D$ pattern and the position of (200) peak.

The particle size $(d)$ is determined using the Scherrer equation: $d=$ $0.9 \lambda / \beta \cos \theta$, where $\beta$ is the full-width at half-maximum, and $\theta$ is the Bragg angle. The calculations yield a size of approximately $61 \mathrm{~nm}$ and $38 \mathrm{~nm}$ for $\mathrm{CsPbBr}_{3}$ and $\mathrm{CsPbBr} r_{2} I_{1}$, respectively.

\subsubsection{FESEM Analysis}

FESEM images of $\mathrm{CsPbBr}_{3}$ and $\mathrm{CsPbBr}_{2} I_{1}$ (Figure 7.9) indicate the formation of films with an irregular surface coverage. The presence of voids might originate from the evaporation of the organic solvent during thermal annealing. The deposited films exhibit grains with a relatively small size $(200-300 \mathrm{~nm})$ with clear grain boundaries. 

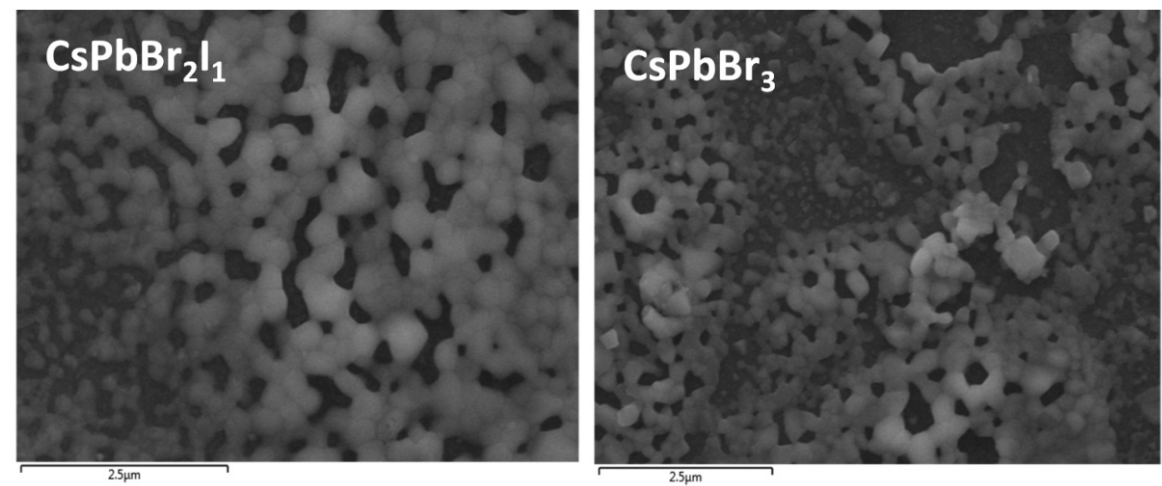

Figure 7.9: FESEM micrographs of a) $\mathrm{CsPbBr}{ }_{2} I_{1}$ and b) $\mathrm{CsPbBr}_{3}$ thin films.

\subsection{3 $U V$-vis Spectra and Luminescence Properties}

As shown in Figure 7.10, the composition of the mixed halide perovskite thin films can be distinguished by their colors, changing from brown $\left(\mathrm{CsPbBr}_{2} I_{1}\right)$ to yellow $\left(\mathrm{CsPbBr}_{3}\right)$. Optical measurements show that the absorbance onset gradually shifts to longer wavelengths as the $I$ concentration increases.

An approximation of the bandgap of $C s \mathrm{PbBr}_{3-x} I_{x}$ was obtained from the maximum of the absorbance, that is closed to the $P L$ peak. These values have been plotted as a function of $x$ for $C s P b B r_{3-x} I_{x}$ (Figure 7.11). The evolution of the bandgap with the iodide fraction deviates slightly from the linear dependence, displaying a downward bowing that can be fitted to a quadratic expression in $x$ :

$$
E g(x)=2.3836-0.2113 x+0.0006 x^{2}
$$

The dependence of the absorption edge with the iodide content can be 
described by the following equation:

$$
E g(x)=E g\left(C s P b B r_{3}\right)+\left[E g\left(C s P b I_{3}\right)-E g\left(C s P b B r_{3}\right)-b\right] \frac{x}{3}+b\left(\frac{x}{3}\right)^{2}
$$

where $b$ is the bowing parameter $[231,264]$ reflecting, on the one hand, the fluctuation degree in the crystal field and, on the other hand, the nonlinear effect arising from the anisotropic nature of binding [232]. The energies extracted from this data analysis were $\mathrm{Eg}\left(\mathrm{CsPbBr}_{3}\right)=2.38 \mathrm{eV}$, $E g\left(\mathrm{CsPbBr}_{2} I_{1}\right)=2.17 \mathrm{eV}, \mathrm{Eg}\left(\mathrm{CsPbI}_{3}\right)=1.75 \mathrm{eV}$ and $b=0.00567 \mathrm{eV}$. The calculated bandgap values are in good agreement with experimental and theoretical values reported $\left(E g\left(\mathrm{CsPbI}_{3}\right)=1.75 \mathrm{eV}, \mathrm{Eg}\left(\mathrm{CsPbBr}_{3}\right)=\right.$ $2.38 \mathrm{eV}$ ) [265]. The relatively small $b$ parameter confirms that compositional disorder is very low in our case.

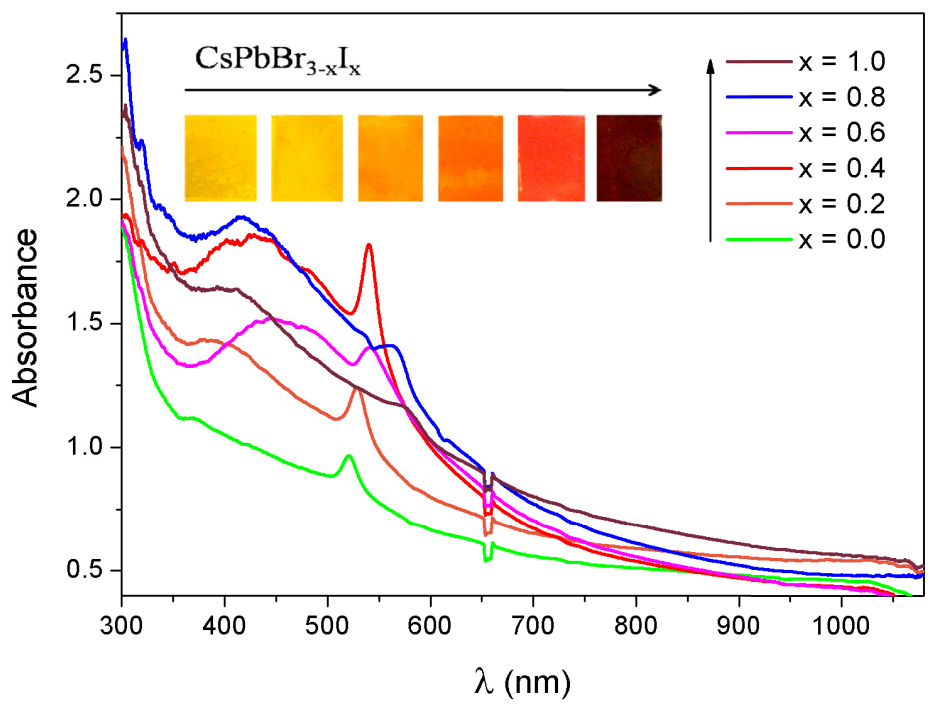

Figure 7.10: $U V$-vis absorption spectra of $C s P b B r_{3-x} I_{x}$ with $(0 \leq x \leq 1)$. Inside, photographs of lead bromide perovskite thin films under ambient light are shown. 
The absorption and $P L$ spectra of $\mathrm{CsPbBr}_{3}$ are shown in Figure 7.12, and as can be seen, the $P L$ spectrum fits perfectly to a Gaussian curve with a FWHM of about $26 \mathrm{~nm}$, indicating a highly pure green emission. This narrow $P L$ spectrum corresponds to the excitonic emission. The $P L$ emission peak, centered at approximately $530 \mathrm{~nm}$, exhibits a low stoke shift from the corresponding absorption spectrum, indicating the existence of a small vibronic relaxation of the $C s P b B r_{3}$ crystal [266]. The value of $P L$ emission is in a good agreement with previous reports [267].

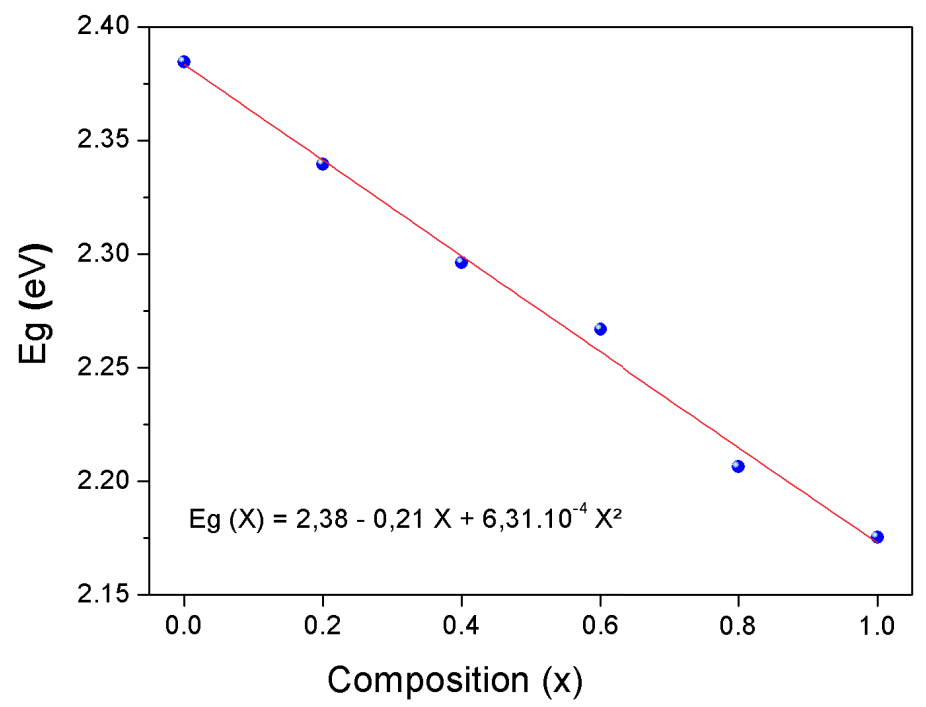

Figure 7.11: Variation of the absorption edge with the chemical composition of bromide for $\mathrm{CsPbBr}_{3-x} I_{x}(0 \leq x \leq 1)$.

As mentioned in the introduction, one of the important characteristics when designing a solar cell is the long-term stability of the materials. For this reason, an investigation into the thermal and the atmospheric stability of cesium based thin films was carried out by studying the impact of humidity and thermal annealing on the optical absorbance of $C s P b B r_{3-x} I_{x}$. 


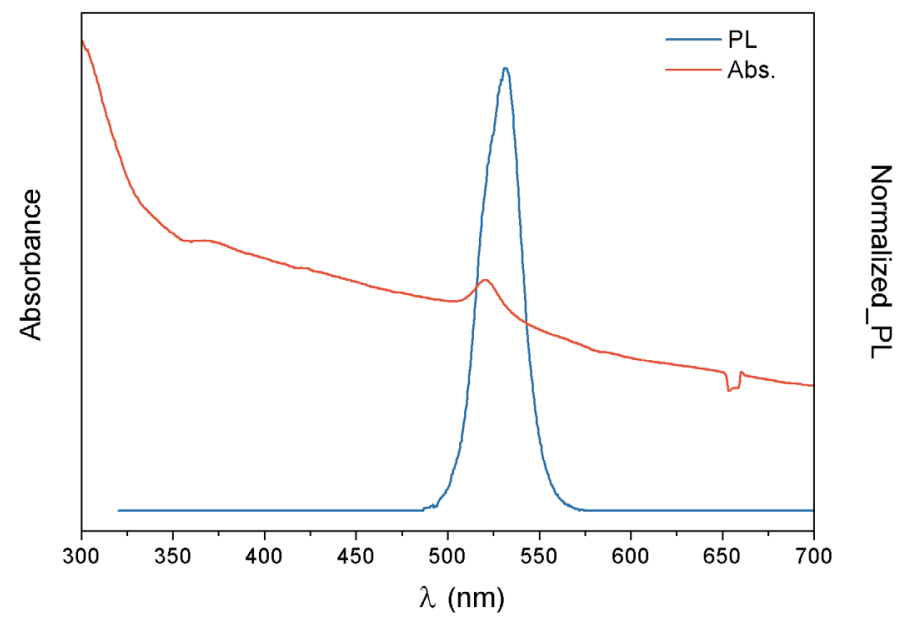

Figure 7.12: $U V$-vis absorption and $P L$ spectra of $C s P b B r_{3}$.

Figures 7.13, 7.14 and 7.15 show the absorbance spectrum of $C s P b B r_{3-x} I_{x}$ $(x=0,0.2,0.6)$ films before and after aging for 2 days under dark conditions at $60 \% \mathrm{RH}$ and after thermal annealing at $180^{\circ} \mathrm{C}$ for $15 \mathrm{~min}$. The results indicate that the absorption onset of $\mathrm{CsPbBr}_{3-x} I_{x}$ is stable with a slight change in the absorbance intensity, especially for $\mathrm{CsPbBr}_{2.4} I_{0.6}$ films. In addition, there was no change in the color of the $\mathrm{CsPbBr}_{3-x} I_{x}$ films after aging for 2 days in ambient atmosphere with $R H$ as high as approximately $60 \%$ or after being heated at high temperature. These results confirm the excellent stability of mixed cesium lead bromide films when compared to $M A$ or $F A$ based perovskite films [268, 269].

An analogous behavior was reported by Kulbak et al. demonstrating that replacing the organic cation of $A M X_{3}$ perovskite structure by an inorganic one, such as cesium, leads to thermally more stable perovskite structure. Since lifetime of $C s \mathrm{PbBr}_{3-x} I_{x}$ based devices is improved, compared to $\mathrm{MAPbBr}_{3}$ based devices [270], mixed cesium lead halide perovskite thin films represent a promising photovoltaic material. 


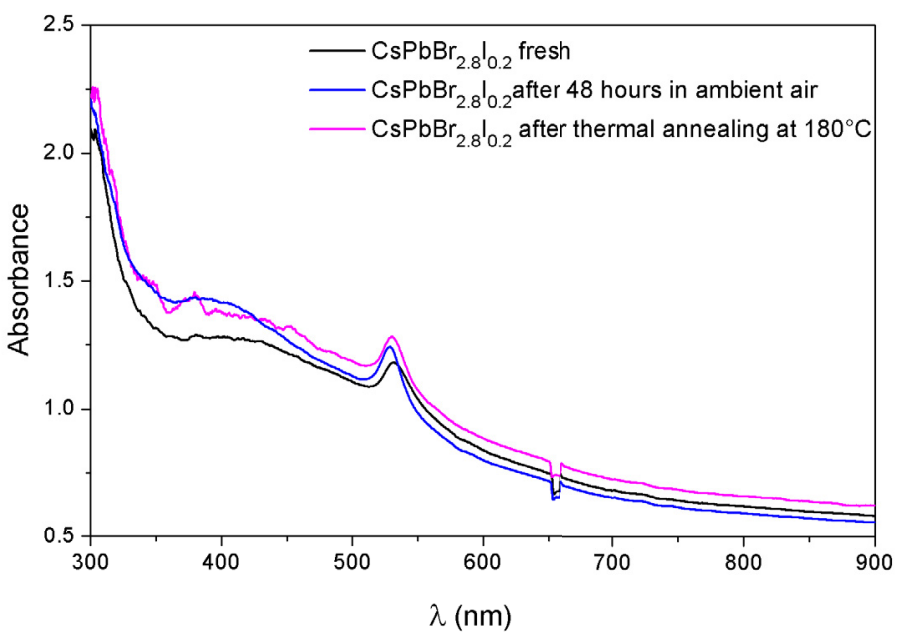

Figure 7.13: Absorbance spectra for $\mathrm{CsPbBr}_{2.8} I_{0.2}$ thin film fresh, after aging for 2 days at $60 \% \mathrm{RH}$ and after a thermal annealing at $180^{\circ} \mathrm{C}$ for 15 min.

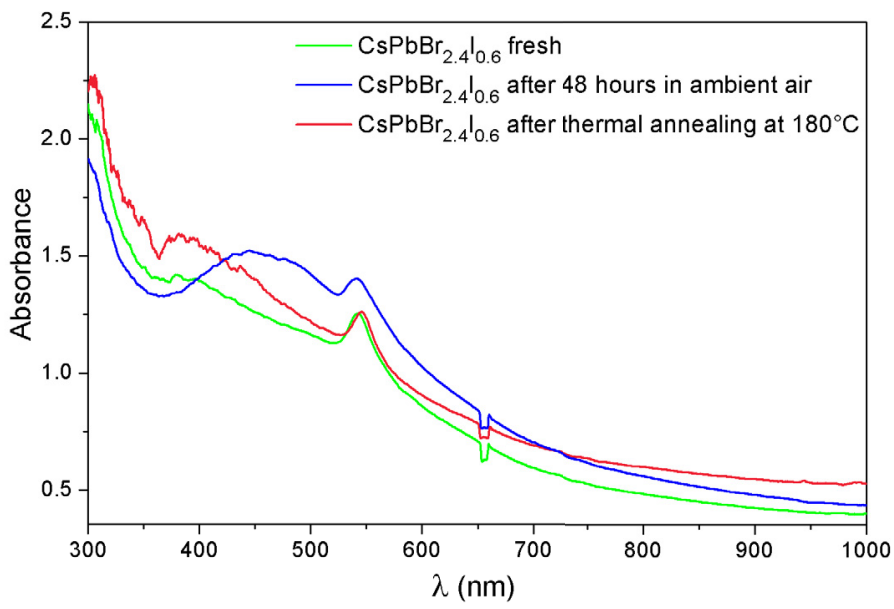

Figure 7.14: Absorbance spectra for $\mathrm{CsPbBr}_{2.4} I_{0.6}$ thin film fresh, after aging for 2 days at $60 \% \mathrm{RH}$ and after a thermal annealing at $180^{\circ} \mathrm{C}$ for 15 min. 


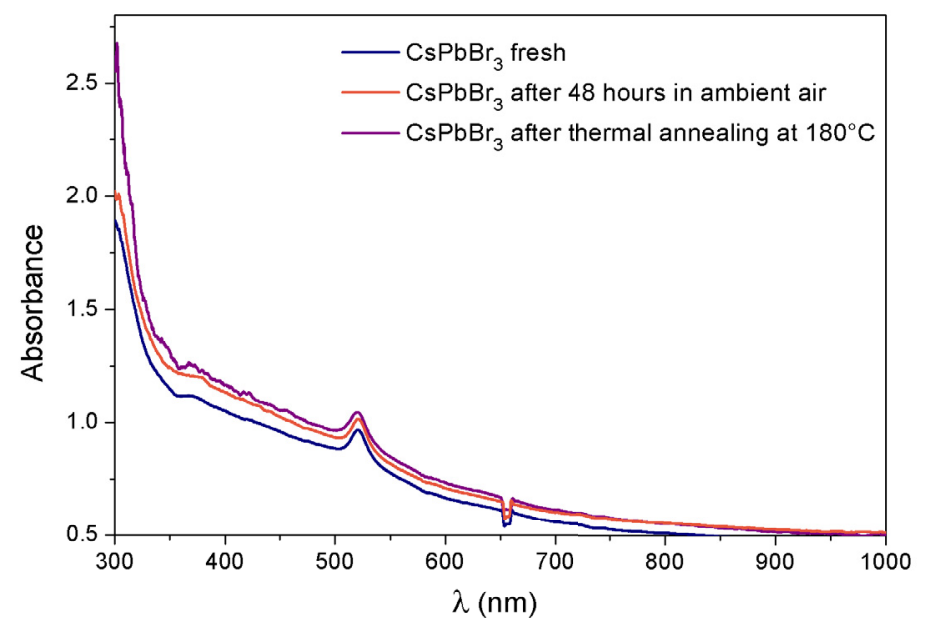

Figure 7.15: Absorbance spectra for $\mathrm{CsPbBr}_{3}$ thin film fresh, after aging for 2 days at $60 \% \mathrm{RH}$ and after a thermal annealing at $180^{\circ} \mathrm{C}$ for $15 \mathrm{~min}$.

\subsection{Conclusion}

The structural and optical properties of solution processed mixed cesium lead perovskite thin films $\operatorname{CsPbBr}_{3-x} I_{x}(0 \leq x \leq 1)$ and their stability were studied when exposed to ambient air and heat. $X$-ray diffraction analyses showed a shift to lower angles when the average fraction of iodide, incorporated into the lattice, increased. Absorbance measurement shows that $\mathrm{CsPbBr} r_{3-x} I_{x}$ films exhibit a very high absorbance in the visible and short infrared region. The bandgap of $\mathrm{CsPbBr}_{3-x} I_{x}$ is tuned from $2.38 \mathrm{eV}\left(\mathrm{CsPbBr}_{3}\right)$ to $2.17 \mathrm{eV}$ $\left(\mathrm{CsPbBr}{ }_{2} I_{1}\right)$ following Vegard's law. In addition, the color of perovskite films can be tuned from yellow to dark brown with increasing the iodide content in mixed halide perovskites. The $P L$ emission of the $\mathrm{CsPbBr}_{3}$ sample indicates a high-purity green emission located at $530 \mathrm{~nm}$. In addition, $\mathrm{CsPbBr}_{3-x} \mathrm{I}_{x}$ showed improved resistance to humidity and heat. These results indicate that mixed cesium lead halide perovskites represent a promising photovoltaic material. 



\title{
Chapter 8
}

\section{Effect of Guanidinium on the}

\author{
Optical Properties and
}

\section{Structure of the}

\section{Methylammonium Lead}

\section{Halide Perovskite}

\subsection{Motivation}

Defect energy levels have been shown to be situated relatively shallow $[279,280]$ and although it had been hypothesized that grain boundaries do not contribute significantly to recombination losses in perovskites, it has been shown that they are much more harmful than previously assumed [281,282]. This means that if it were possible to mitigate nonradiative carrier losses, the efficiency of perovskite solar cells could be substantially improved. In order to successfully mitigate such charge carrier losses, recombination pathways 
within the perovskite and device have been suppressed. Employing additives in perovskite methylammonium lead iodide seems to be an efficient method to tune its properties such as defect density and charge carrier lifetime. Regarding this topic, under-coordinated ions that may exist at grain boundaries and surfaces that can act as charge carrier trap/recombination centers $[283,284]$ have been described.

Different passivation methods have been employed up to now. For example, an iodopentafluorobenzene (IPFB) post-treatment has been used to passivate under-coordinated iodine [283], thiophene and pyridine have been employed to passivate under-coordinated $\mathrm{Pb}$ ions [284] and, recently, $\mathrm{Br}$ and $\mathrm{Cl}$ have been suggested to assist in suppressing recombination and decoupling electron-hole pairs $[285,286]$.

In order to improve performance, the guanidinium organic molecule $\mathrm{CH}_{6} \mathrm{~N}_{3}^{+}(G A)$ has been investigated as an additive for dye-sensitized solar cells $(D S S C s)$, in which it serves to passivate $\mathrm{TiO}_{2}$ surfaces. Also, it has been studied as an additive in $\mathrm{MAPbI}_{3}$ in order to improve structure, stability and performance. $G A$ has a larger size $(278 \mathrm{pm})$ than $M A(217 \mathrm{pm})$, which can lead to different perovskite structures and/or behaviors [109,287], therefore it is necessary to investigate the consequences of the GA addition in detail. Some authors suggest that bias-induced motion and hysteresis effects might be influenced by the zero-dipole moment of $G A[288,289]$.

Very recently, a passivation effect through partial $G A$ incorporation in perovskite films has been demonstrated [288], obtaining a significant mitigation of nonradiative decay and an enhanced carrier lifetime, high open circuit voltages and improved device performances.

In this chapter, the structural and optical effects of partial $G A$ incorporation in $\mathrm{MAPbI}_{3}$ perovskite thin films, with the aim of increasing 
and improving the stability of solar cells, is presented.

\subsection{Experimental procedure}

Guanidinium iodine $\left(\mathrm{CH}_{6} \mathrm{~N}_{3} I, G A I\right)$ was purchased from Sigma Aldrich. Crystalline methylammonium iodide $\left(\mathrm{CH}_{3} \mathrm{NH}_{3} \mathrm{I}, \mathrm{MAI}\right)$ powder was synthesized by reacting $0.3 \mathrm{~mol}$ of concentrated hydriodic acid (Sigma Aldrich) with $0.3 \mathrm{~mol}$ of methylamine $\left(\mathrm{CH}_{3} \mathrm{NH}_{2}\right)$ solution (33\% in methanol, Sigma Aldrich) whilst being stirred for $2 h$ in a $250 \mathrm{~mL}$ round-bottom flask kept in an ice bath $\left(0^{\circ} \mathrm{C}\right)$. The solvent was evaporated at $50^{\circ} \mathrm{C}$ in the rotary evaporator. Then, the precipitate was filtered and recrystallized by solving it in absolute ethanol and precipitating it with the addition of diethyl ether. After this process, pure $M A I$ was obtained.

The solutions $M A_{1-x} G A_{x} P b I_{3}$ for $0 \leq x \leq 1$ were prepared under inert atmosphere in a glove box dissolving the appropriate amount of the $M A I$ and $G A I$ powders with $\mathrm{PbI}_{2}(99 \%$ Sigma Aldrich) at $45 \%$ wt in $D M F$ (Dimethylformamide). The solutions were stirred at $60^{\circ} \mathrm{C}$ for 30 minutes.

The films were deposited onto conductive glass substrates ITO (Indium Tin Oxide) using the spin coating process at a speed of $5000 \mathrm{rpm}$ for 20 seconds. The $\mathrm{MAPbI}_{3}$ samples were annealed for $1 \mathrm{~h}$ at $100^{\circ} \mathrm{C}$ and the $M A_{1-x} G A_{x} \mathrm{PbI}_{3}$ thin films were annealed at $135^{\circ} \mathrm{C}$ for 15 minutes.

\subsection{Results and Discussion}

\subsubsection{X-Ray Diffraction}

Figure 8.1 presents the $X$-ray diffractograms of $M A_{1-x} G A_{x} \mathrm{PbI}_{3}$ for different $x$ values. The $M A P b I_{3}$ perovskite characteristic peaks are located at approximately $14.1^{\circ}$ and $28.4^{\circ}(2 \theta)$, corresponding to planes (100) and (200), 
respectively. These peaks can be observed for the $M A_{1-x} G A_{x} P b I_{3}$ samples $(0 \leq x \leq 0.2)$. When the $G A$ content arrives at $20 \%$, the peaks located at $8.4^{\circ}$ and $11.2^{\circ}(2 \theta)$, corresponding to the $G A$ tetragonal phase become visible, indicating the solubility limit has been reached. To determine the lattice parameter of $G A P b I_{3}$, a Pawley refinement of the $X R D$ spectra was carried out. A tetragonal lattice type (space group $P 42 / n m c)$ was found when neglecting the ITO peaks. Figure 8.2 shows a good fit between the simulated and the experimental diffractograms.

In order to gain further insight into the $G A$ incorporation, Pawley refinements of the $M A_{1-x} G A_{x} \mathrm{PbI}_{3}(0 \leq x \leq 0.2) X R D$ patterns were carried out obtaining a tetragonal lattice type (space group $14 \mathrm{~cm}$ ) and experimental and simulated data for the 100 peak are shown in Figure 8.3.

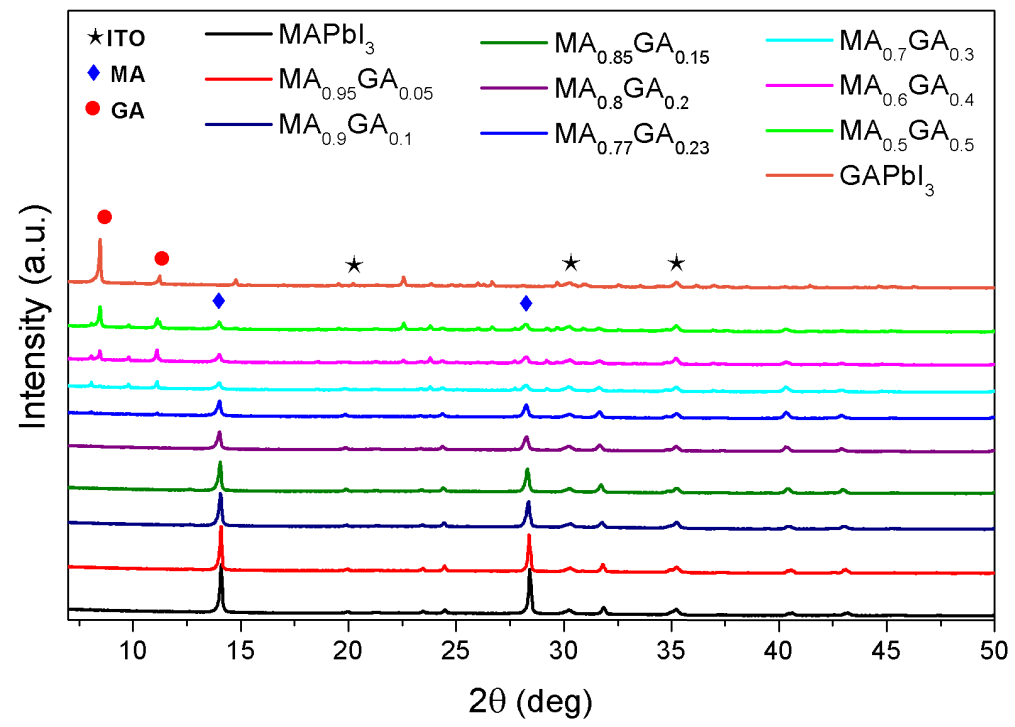

Figure 8.1: $X$-ray diffractograms of $M A_{1-x} G A_{x} \mathrm{PbI}_{3}(0 \leq x \leq 1)$. 


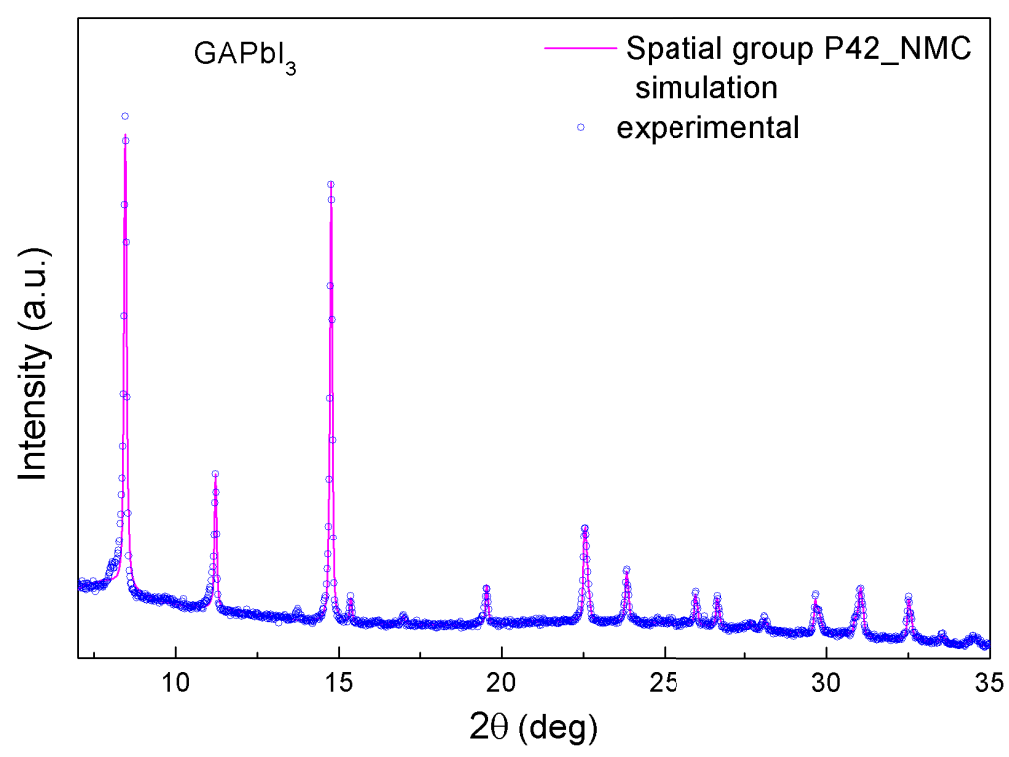

Figure 8.2: Pawley refinement of the $X R D$ spectra for pure $G A P b I_{3}$.

Table 8.1 Refinement factors and refined cell parameters using the Pawley method.

\begin{tabular}{lccc}
\hline Sample & $a=b(\AA)$ & $c(\AA)$ & $R w p$ \\
\hline$M A P b I_{3}$ & 8.87659 & 12.65859 & $4.42 \%$ \\
$M A_{0.9} G A_{0.1} \mathrm{PbI}_{3}$ & 8.90393 & 12.66932 & $4.21 \%$ \\
$\mathrm{MA}_{0.8} \mathrm{GA}_{0.2} \mathrm{PbI}_{3}$ & 8.93066 & 12.61813 & $3.95 \%$ \\
$\mathrm{GAPbI}_{3}$ & 11.97202 & 20.86364 & $5.52 \%$ \\
\hline
\end{tabular}

The addition of $G A$ produces a slight shift toward a lower angle of the characteristic (100) and (200) $M A$ perovskite peaks, located at $14.1^{\circ}$ and at $28.4^{\circ}$, which can be attributed to the fact that $G A$ has a larger volume than $M A$ and the partial substitution produces an increase in the lattice parameter. Table 8.1 summarizes the results of the Pawley refinements. Figure 8.4 shows the evolution of the lattice parameters vs $G A$ content $x$. As can be seen, the cell parameter $a$ varies almost linearly, following Vegard's law. However, 
parameter $c$ slightly increases with a $G A$ content of up to $10 \%$. The abrupt decrease for higher concentrations than $x=0.15$ indicates a probable lattice change.

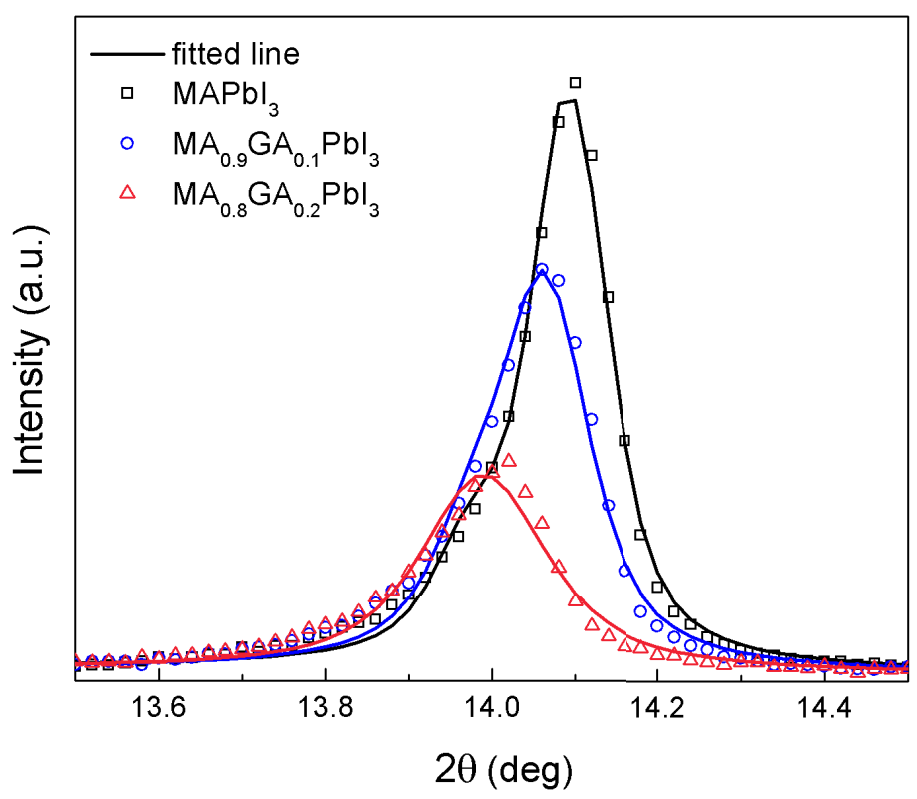

Figure 8.3: $X R D$ patterns of the $M A P b I_{3}$ and $M A_{1-x} G A_{x} P b I_{3}$ with $x=0$, $0.1,0.2$.

In order to evaluate the stability of mixed perovskite samples, diffractograms of $M A P b I_{3}$ and $M A_{1-x} G A_{x} P_{b I}$ thin films were recorded immediately after sample preparation. These samples were kept at room temperature and humidity for eight months before a control diffractogram was made.

Figures 8.5, 8.6, 8.7 and 8.8 show diffractograms for $M A_{1-x} G A_{x} \mathrm{PbI}_{3}(0 \leq$ $x \leq 0.15)$ fresh and after eight months aging, denoted $(A)$ and $(B)$ in each graph. As can be seen, the aged pure methylammonium perovskite shows a $12.6^{\circ}$ peak, corresponding to $\mathrm{PbI}_{2}$, which evidences perovskite decomposition. 


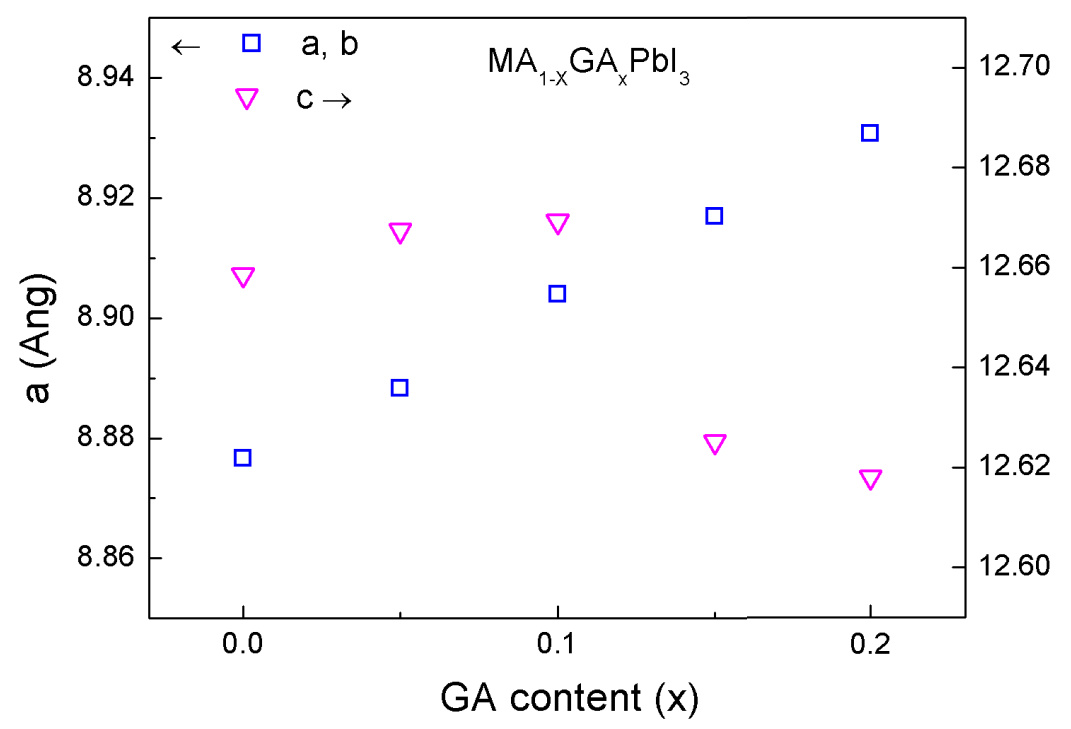

Figure 8.4: Lattice parameters as a function of $G A$ content.

In the $M A_{0.95} G A_{0.05} \mathrm{PbI}_{3}$ sample (Figure 8.6) it is observed a low intensity peak corresponding to the presence of $\mathrm{PbI}_{2}$ in both fresh and after eight months aging diffractograms. This may be due to small amounts of not properly dissolved $\mathrm{PbI}_{2}$, leaving impurities in the solution. However, the peak corresponding to $\mathrm{PbI}_{2}$ does not show larger intensity after 8 months aging, which can be interpreted as that the sample has not suffered degradation. On the other hand, the results after aging show that the sample $M A_{0.9} G A_{0.1} P b I_{3}$ remains unaltered (Figure 8.7). Finally, $X R D$ for $M A_{0.85} G_{0.15} \mathrm{PbI}_{3}$ (Figure 8.8) showed $\mathrm{PbI}_{2}$ and/or $\mathrm{GA}$ peaks that evidence decomposition or guanidinium perovskite segregation after aging. 


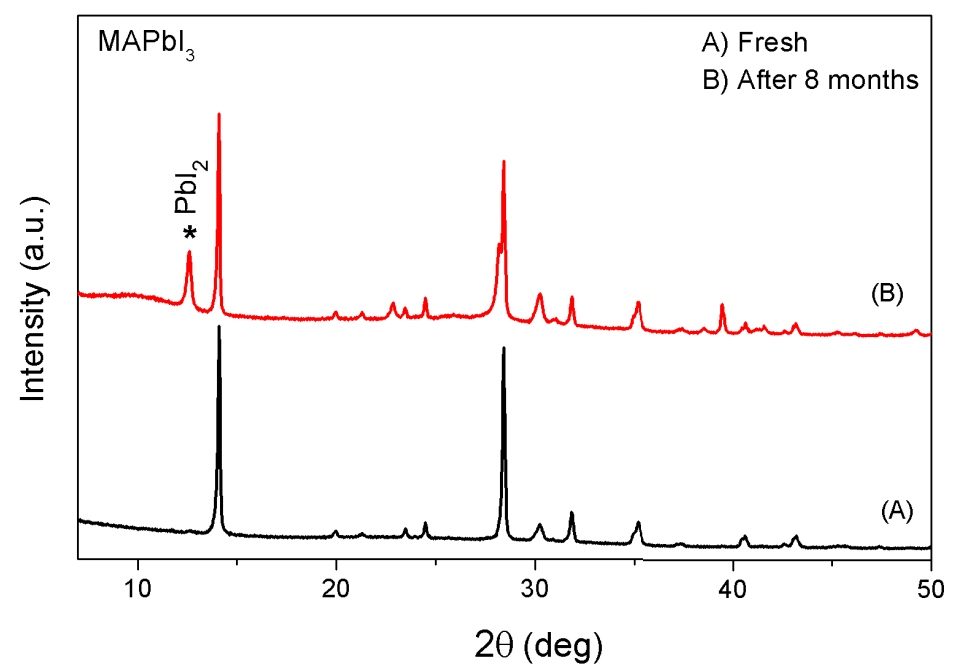

Figure 8.5: $\mathrm{XRD}$ for $\mathrm{MAPbI}_{3}$ fresh and after eight months.

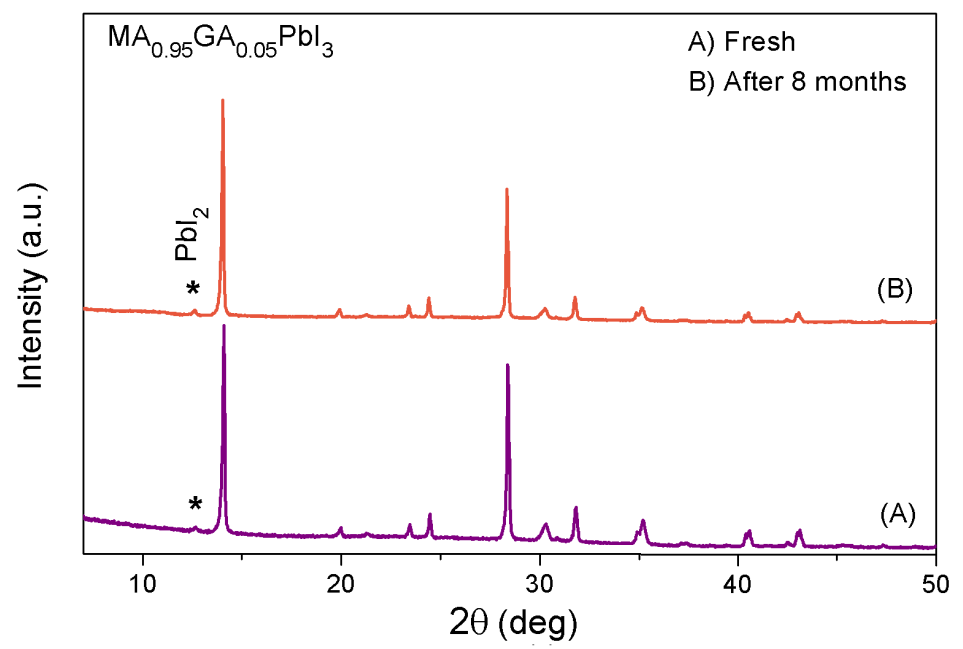

Figure 8.6: $X R D$ for $M A_{0.95} G A_{0.05} \mathrm{PbI}_{3}$ fresh and after eight months. 


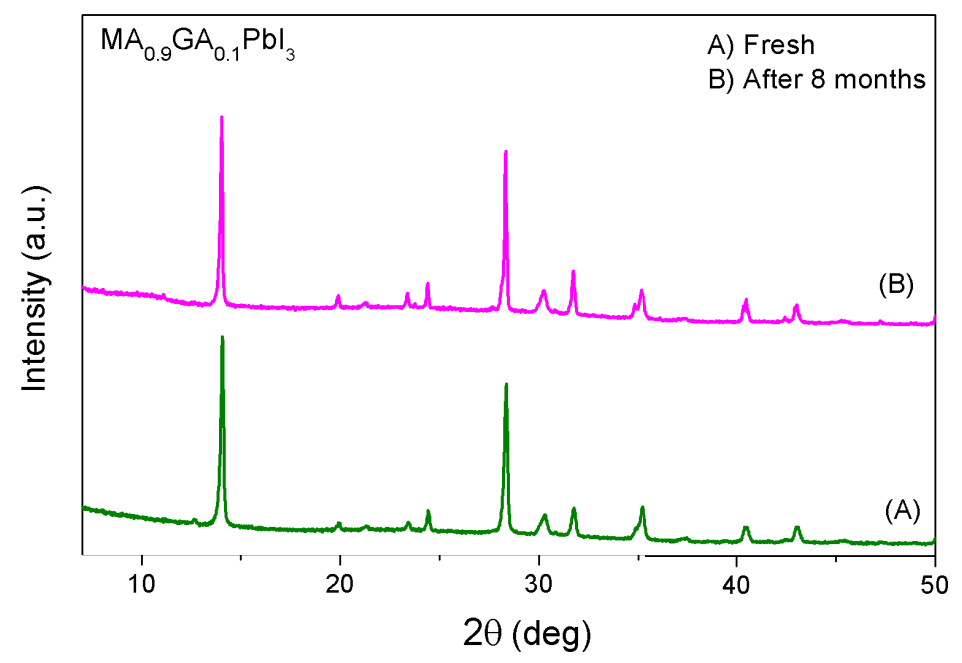

Figure 8.7: $X R D$ for $M A_{0.9} G A_{0.1} P b I_{3}$ fresh and after eight months.

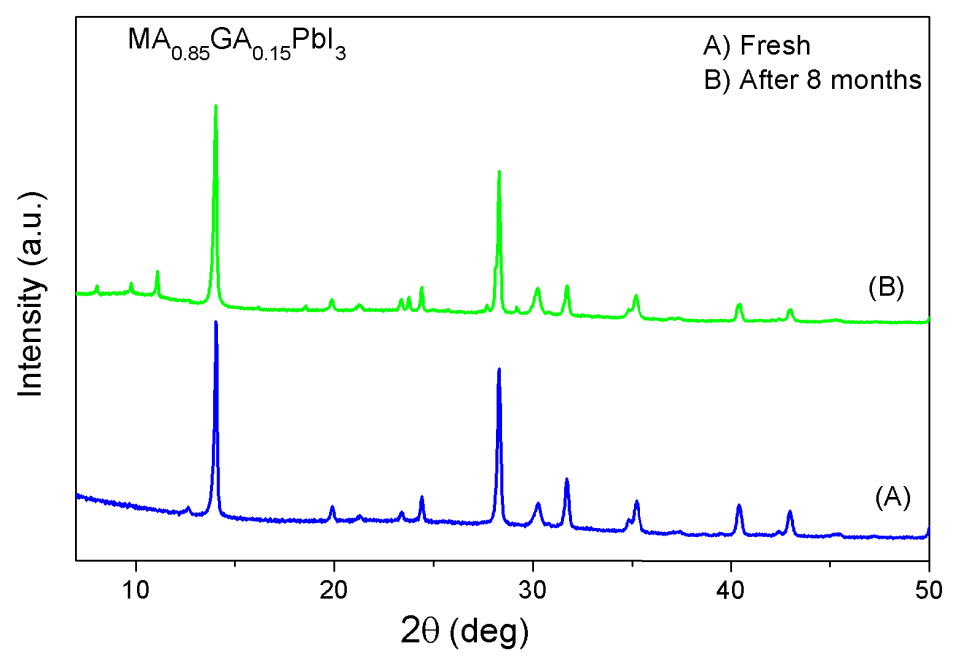

Figure 8.8: $X R D$ for $M A_{0.85} G A_{0.15} \mathrm{PbI}_{3}$ fresh and after eight months.

\subsubsection{Absorbance}

Figure 8.9 shows a change of color varying from black for $\mathrm{MAPbI}_{3}$ to yellow for $\mathrm{GAPbI}_{3}$. An integrating sphere was used to collect both, specular 
and diffuse transmittance to reduce the effect of light scattering originated from refraction and reflection phenomena on the perovskite crystals. The absorption spectra for different $G A$ values are also shown in Figure 8.9, where a decrease of the slope for a $G A$ contents above $20 \%$ can be appreciated, indicating a degradation of the bandgap. This result agrees with the previously suggested lattice change. The inset of Figure 8.9 shows the absorbance for the $\mathrm{GAPbI}_{3}$ sample.

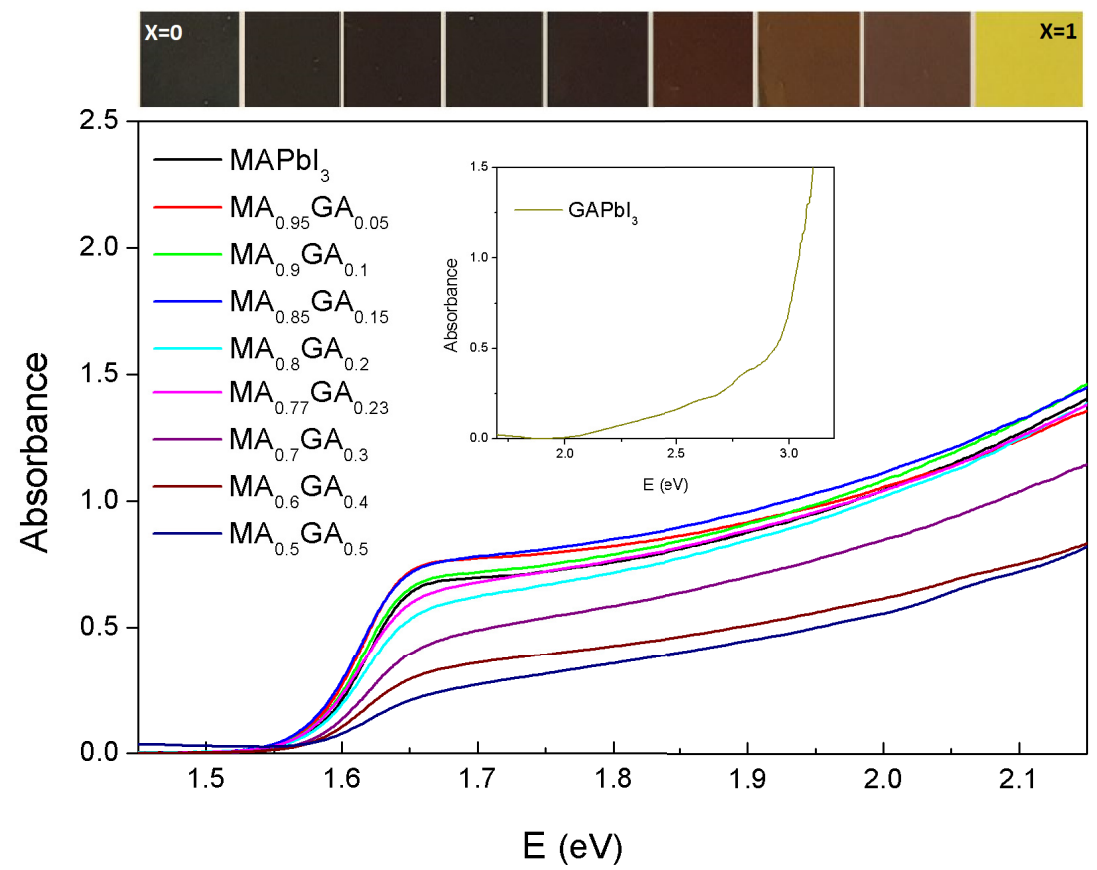

Figure 8.9: Thin film images of $M A_{1-x} G A_{x} P b I_{3}$ for $0 \leq x \leq 1$, from left to right, and $U V$-vis absorption spectra of $M A_{1-x} G A_{x} P b I_{3}$ for different $G A$ contents.

The $G A$ fraction contained in $M A_{1-x} G A_{x} P b I_{3}$ affects the optical properties. It can easily be appreciated that an increasing amount of $G A$ results in front edge diminution, and therefore, a reduction of the absorptance. Figure 8.10 provides Tauc plots showing variations in the optical bandgap. It 
can be observed that for the samples between 0 and $20 \%$, the bandgap evolves very slightly towards lower energies.

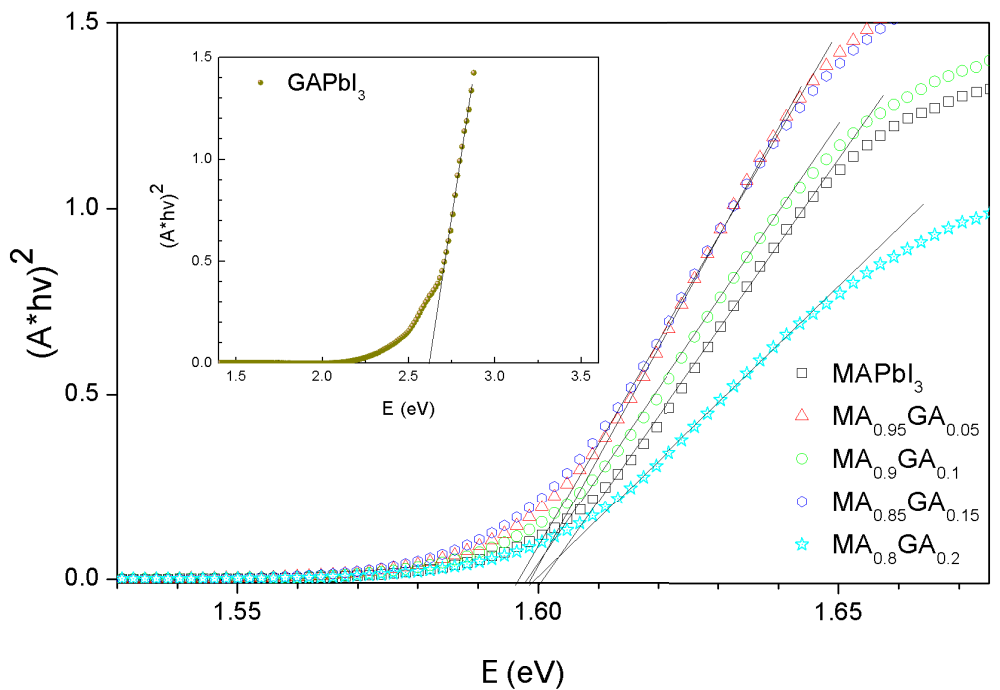

Figure 8.10: Tauc plots showing variations in optical bandgap.

Table 8.2 Bandgap variation depending on $G A$ content.

\begin{tabular}{lcc}
\hline Sample & \multicolumn{2}{c}{ Absorption edge at room $\mathrm{T}$} \\
\cline { 2 - 3 } Composition & $(\mathrm{eV})$ & $(\mathrm{nm})$ \\
\hline$M A P b I_{3}$ & 1.60 & 775 \\
$\mathrm{MA}_{0.95} \mathrm{GA}_{0.05} \mathrm{PbI}_{3}$ & 1.59 & 780 \\
$\mathrm{MA}_{0.9} \mathrm{GA}_{0.1} \mathrm{PbI}_{3}$ & 1.59 & 780 \\
$\mathrm{MA}_{0.85} \mathrm{GA}_{0.15} \mathrm{PbI}_{3}$ & 1.59 & 780 \\
$\mathrm{MA}_{0.8} \mathrm{G} A_{0.2} \mathrm{PbI}_{3}$ & 1.59 & 780 \\
$\mathrm{GAPbI}$ & 2.62 & 470
\end{tabular}

Table 8.2 shows the estimated bandgap $\left(E_{g}\right)$ of $M A_{1-x} G A_{x} P b I_{3}$, for samples between 0 and $20 \% G A$ contents obtained from the Figure 8.10. It 
can be seen that the bandgap remains almost constant, the overall variation between all the samples being 1.59 to $1.60 \mathrm{eV}$, corresponding to absorption edges spanning 775-780 $\mathrm{nm}$. The inset of Figure 8.10 shows $E_{g}$ for the $\mathrm{GAPbI}_{3}$ sample being $2.62 \mathrm{eV}$, corresponding to an absorption edge of $470 \mathrm{~nm}$.

\subsubsection{Photoluminescence}

The photoluminescence study at different temperatures illustrates charge carrier dynamics as well as the quality of the material emission. Figure 8.11 shows the $P L$ results of $M A_{1-x} G A_{x} P b I_{3}(x=0,0.15,0.5,1)$ at $11 \mathrm{~K}$. Intensities have been normalized to clarify the shift of the emission peaks. It can easily be observed that the position of the peak about $800 \mathrm{~nm}$ for the $M A$-GA-based samples shifts slightly to higher wavelengths as the $G A$ concentration increases. This behavior could be explained by the bandgap defects introduced by the $G A$ doping. Further research is required to clarify this behavior. Moreover, a pure $G A P b I_{3}$ perovskite shows a single peak located at about $565 \mathrm{~nm}$.

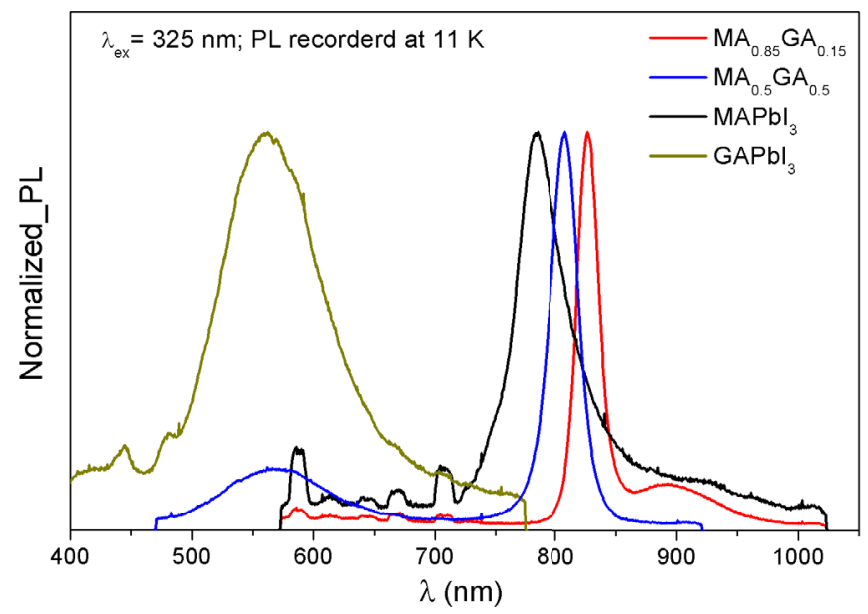

Figure 8.11: Normalized $P L$ spectra of $M A_{1-x} G A_{x} P b I_{3}$ for $0 \leq x \leq 1$ at $11 \mathrm{~K}$. 
For a $G A$ content of $50 \%$, both peaks at 570 and 810 can be observed, which is indicative of the two phases being present, in agreement with previous results for a $G A$ content above $20 \%$.

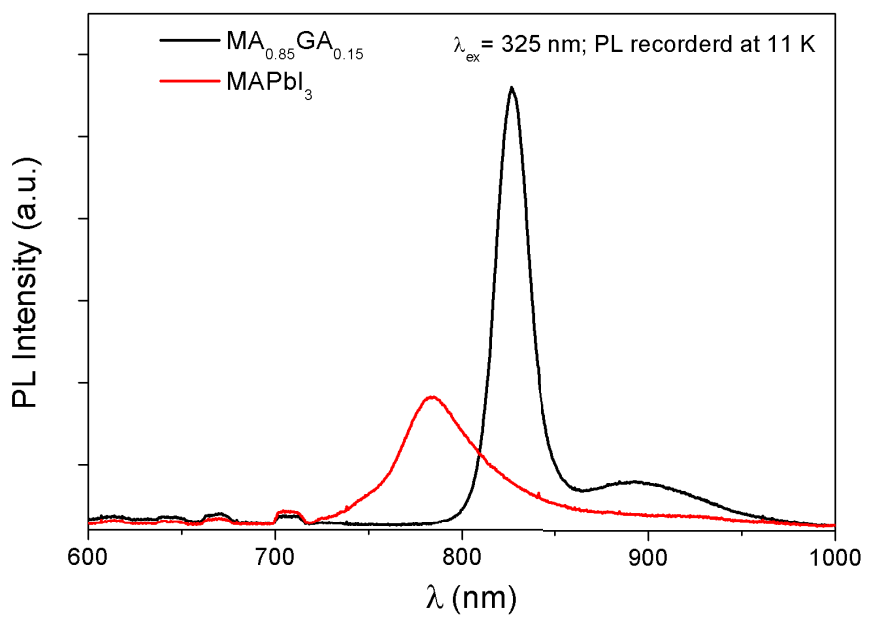

Figure 8.12: $P L$ spectra of $M A P b I_{3}$ and $M A_{0.85} G A_{0.15} P b I_{3}$ at $11 \mathrm{~K}$.

Table 8.3 displays that the calculated bandgap at different temperatures obtained from $P L$ measurements increased as the temperature increased, as can be inferred from the evolution of the peaks towards lower wavelengths. $P L$ intensity of the $M A P b I_{3}$ and $M A_{0.85} G_{0.15} \mathrm{PbI}_{3}$ samples at $11 \mathrm{~K}$ is shown in Figure 8.12. The $M A_{0.85} G A_{0.15} \mathrm{PbI}_{3}$ shows a large rise in $P L$ intensity, more than three times, that of the pure $M A$ samples, in agreement with [286].

The $P L$ spectrum for $M A P b I_{3}$ recorded in heating at different temperatures is shown in Figure 8.13. It can be seen that when temperature increases, the intensity of the peak decreases and, at the same time, the peak shifts to higher wavelengths and, therefore, to lower energies. In Figure 8.14, a magnified $P L$ spectrum is shown, where it is easier to see the shift and the narrowing of the peak as temperature increases. 


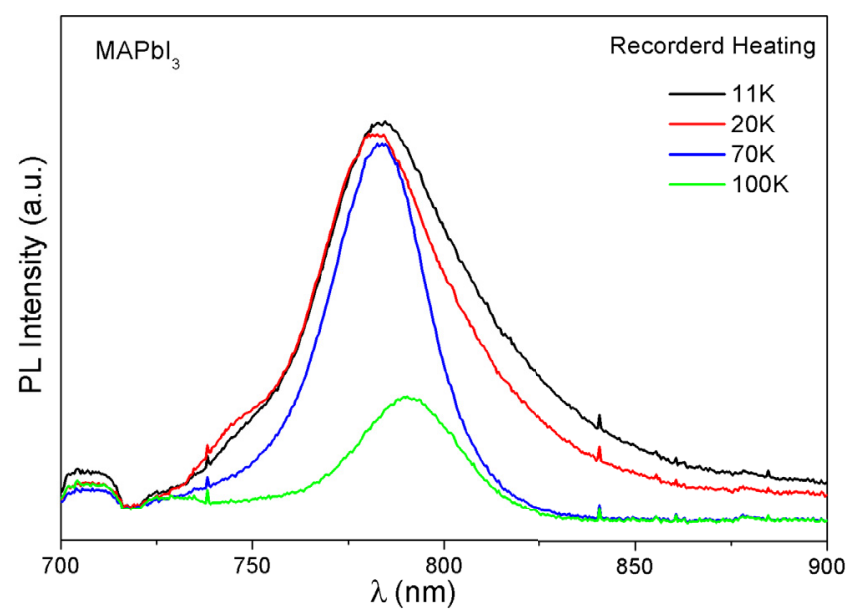

Figure 8.13: $P L$ spectra for $M A P b I_{3}$ in heating at different temperatures.

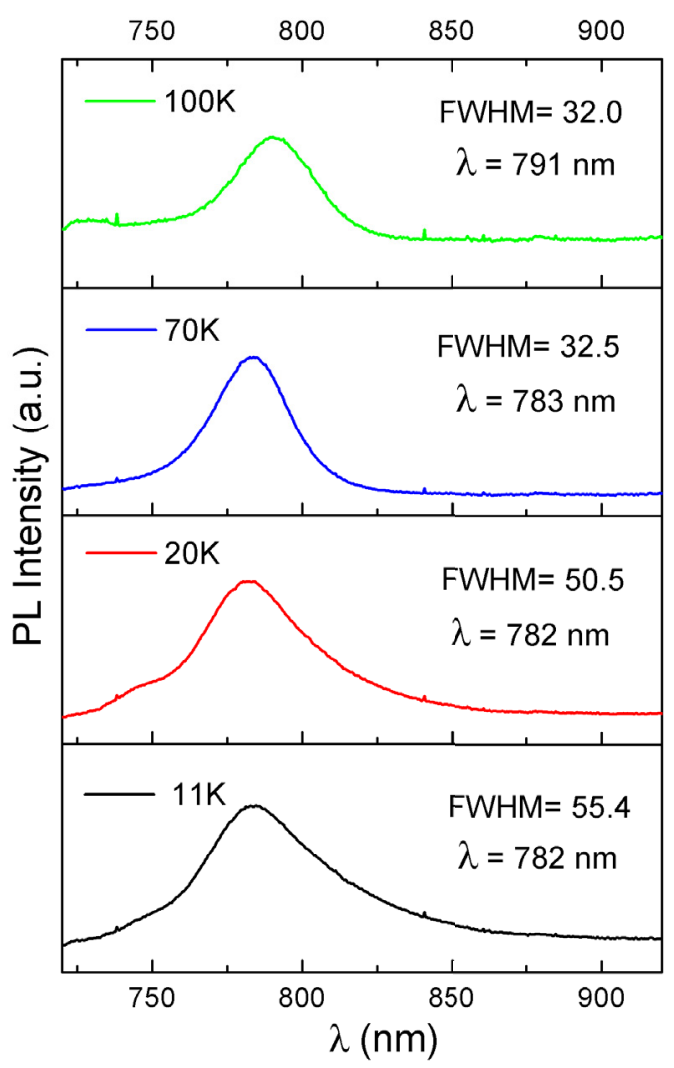

Figure 8.14: Magnified $P L$ spectra and the corresponding $F W H M$ value. 
Table 8.3 Bandgap variation according to temperature and $G A$ content.

\begin{tabular}{lcccccccc}
\hline & \multicolumn{7}{c}{$P L$ peak position } \\
\cline { 2 - 9 } Sample Composition & \multicolumn{2}{c}{$11 \mathrm{~K}$} & \multicolumn{2}{c}{$20 \mathrm{~K}$} & $70 \mathrm{~K}$ & \multicolumn{2}{c}{$100 \mathrm{~K}$} \\
\cline { 2 - 9 } & $(\mathrm{eV})$ & $(\mathrm{nm})$ & $(\mathrm{eV})$ & $(\mathrm{nm})$ & $(\mathrm{eV})$ & $(\mathrm{nm})$ & $(\mathrm{eV})$ & $(\mathrm{nm})$ \\
\hline $\mathrm{MAPbI}_{3}$ & 1.57 & 787 & 1.58 & 785 & 1.58 & 782 & 1.58 & 782 \\
$\mathrm{MA}_{0.85} \mathrm{GA}_{0.15} \mathrm{PbI}_{3}$ & 1.49 & 827 & 1.51 & 818 & 1.52 & 814 & 1.53 & 809 \\
$\mathrm{MA}_{0.5} \mathrm{GA}_{0.5} \mathrm{PbI}_{3}$ & 1.53 & 807 & 1.53 & 807 & 1.56 & 795 & 1.56 & 795 \\
$\mathrm{GAPbI}_{3}$ & 2.19 & 565 & 2.21 & 560 & 2.22 & 558 & - & - \\
\hline
\end{tabular}

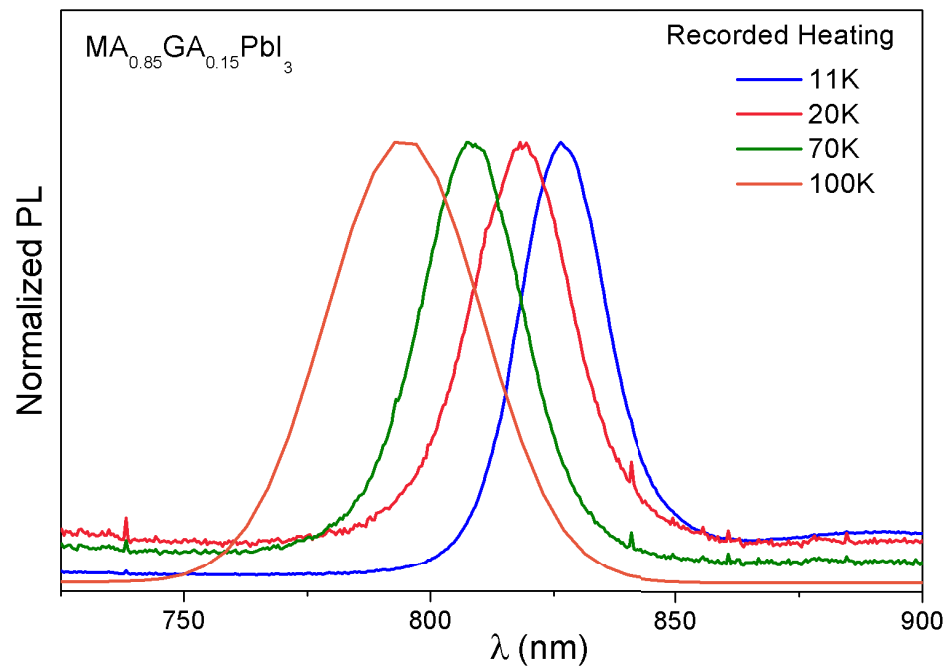

Figure 8.15: Normalized $P L$ spectra for $M A_{0.85} G A_{0.15} P b I_{3}$ in heating at different temperatures.

However, the normalized $P L$ spectrum for $M A_{0.85} G A_{0.15} P b I_{3}$ recorded in heating at different temperatures (Figure 8.15) behaves contrary to the 
$M A P b I_{3}$. In this figure the decreasing of the intensity, as the temperature increases, is not shown since $P L$ spectrum has been normalized with the objective to better shown the displacement of the peak. When temperature increases the peak shifts to lower wavelengths and, thus, to higher energies. The magnified $P L$ spectrum, in Figure 8.16, shows the peak position and the widening of the peak, as temperature increases.

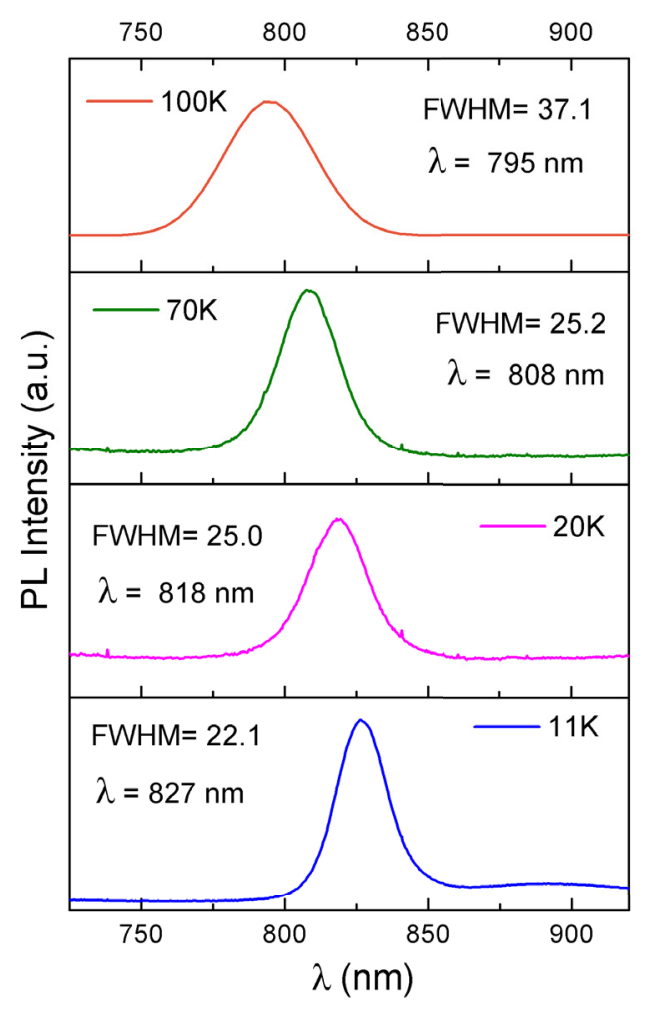

Figure 8.16: Magnified $P L$ spectra and the corresponding $F W H M$ value for $M A_{0.85} \mathrm{GA}_{0.15} \mathrm{PbI}_{3}$.

\subsubsection{FESEM Analysis}

In order to study the impact of $G A$ incorporation on the morphology of thin films, with and without the addition of anti-solvent (chlorobenzene) during the spin-coating process, a FESEM analysis was conducted. 

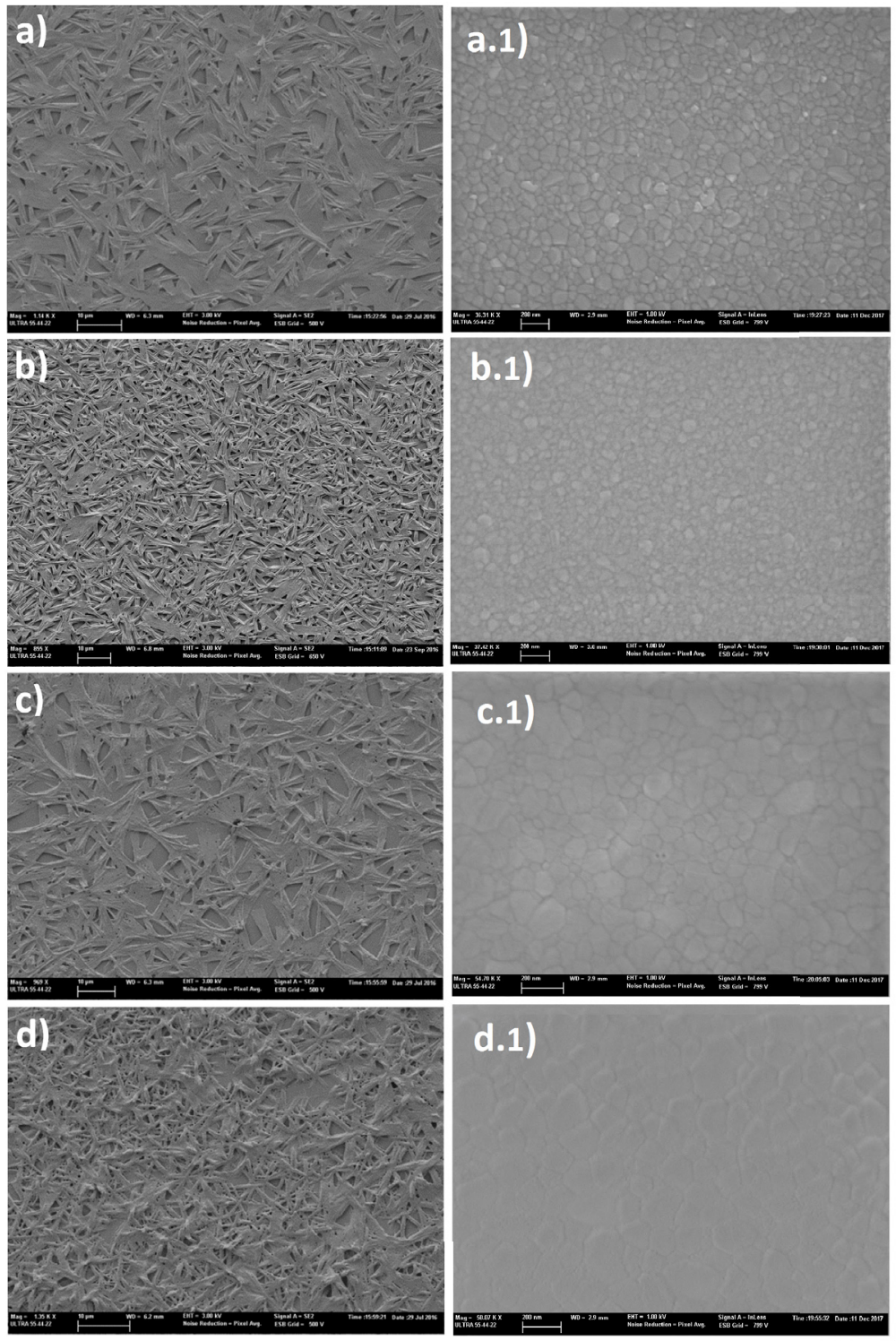

Figure 8.17: FESEM micrographs of (a) $M A P b I_{3}$, (b) $M A_{0.85} G A_{0.15} P I_{3}$, (c) $M A_{0.5} G A_{0.5} \mathrm{PbI}_{3}$ and (d) $\mathrm{GAPbI}_{3}$, on the left without antisolvent and on the right with antisolvent.

Chlorobenzene was chosen based on its high boiling point and good miscibility with $D M F$, since these characteristics have shown good results in the literature. Figure 8.17 shows the top view FESEM images, from top 
to bottom, of $M A P b I_{3}, M A_{0.85} G A_{0.15} P b I_{3}, M A_{0.5} G A_{0.5} P_{b I}$ and $\mathrm{GAPbI}_{3}$ perovskite thin films without anti-solvent (left) and with anti-solvent (right).

As the amount of $G A$ increases, the layers become more homogeneous, although the absorption decreases and loses efficiency. The $M A_{0.85} G A_{0.15} \mathrm{PbI}_{3}$ needle-shape layer can be appreciated, with having a better-covered and smoother surface.

The size of the needle-shape diminishes, increasing the homogeneity, when the amount of $G A$ increases. On the right, the same pattern of improvement can be observed. Nevertheless, a more uniform and smoother grain-shape surfaces can be achieved with the addition of anti-solvent. The grain size increase with the $G A$ content, reaching the maximum size for the $\mathrm{GAPbI}_{3}$ thin film. These results corroborate the importance of employing anti-solvents on the perovskite crystal growth kinetics and film morphology.

\subsection{Conclusions}

This work reports the synthesis of $M A_{1-x} G A_{x} \mathrm{PbI}_{3}$ for different $x$ values under an inert atmosphere, deposited on ITO substrate by spin coating. $\mathrm{X}$-Ray diffraction analysis showed that the $\mathrm{MAPbI}_{3}$ perovskite characteristic peaks were only found in the $M A_{1-x} G A_{x} P b I_{3}$ samples $(0.1 \leq x \leq 0.2)$, indicating the $G A$ incorporation in $M A P b I_{3}$ lattice.

Tetragonal lattice type, space groups $P 42 / \mathrm{nmc}$ and $I 4 \mathrm{~cm}$, were found for $\mathrm{GAPbI}_{3}$ and $\mathrm{MAPbI}_{3}$, respectively. When the $G A$ content exceeds $20 \%$, both $M A P b I 3$ and $\mathrm{GAPbI}_{3}$ peaks were present, indicating the solubility limit is reached.

Absorption spectra show that an increase in the $G A$ content affects the optical properties, reducing the absorptance and therefore the light harvesting capability. 
Samples with a $G A$ content between 0 and $20 \%$ showed a very slight shift towards lower energies, and samples with a $G A$ content above $20 \%$ exhibited a decrease of the slope and, therefore, a degradation of the bandgap. The estimated bandgap $\left(E_{g}\right)$ for the samples with a $G A$ content between 0 and $20 \%$ decreased from 1.60 to $1.59 \mathrm{eV}$, corresponding to absorption edges spanning 775-780 nm.

$P L$ results of $M A_{1-x} G A_{x} P b I_{3}$ for different values of $x$ at different temperatures showed that the position of the peaks of the $G A$-based samples shifts slightly to higher wavelengths as the $G A$ concentration increases. Energy peak decreases can be explained by the bandgap defects introduced by the substitution of $M A$ by $G A$. Above a $G A$ content of $20 \%$, peaks at 570 and $810 \mathrm{~nm}$ can be observed, which confirms that both phases are present.

To study the impact of $G A$ incorporation on the morphology of thin films, with and without the addition of anti-solvent (chlorobenzene) during the spin-coating process, a FESEM analysis was carried out. It has been shown that, as the amount of $G A$ content increases, the thin films become more homogeneous, although the absorption decreases and loses efficiency. The same pattern of improvement can be observed when anti-solvent has been employed, obtaining more uniform and smoother grain-shape surfaces, and corroborating the importance of employing anti-solvents on the perovskite crystal growth kinetics and film morphology. 



\section{Chapter 9}

\section{Improving Structural and \\ Optical Properties via \\ Imidazolium Cation}

\section{Integration on}

\section{Methylammonium Lead}

\section{Iodide Perovskite}

\subsection{Motivation}

As mentioned in the previous chapters, one of the principal problems of $\mathrm{MAPbI}_{3}$ perovskite material is its stability. It has been proved that under humid conditions $M A P b I_{3}$ perovskite materials degrade [223], however $\mathrm{MAPbBr}_{3}$ is less sensitive to humidity [224,225,271] but at the expense of reducing the absorption properties. These results led to the study of mixed 
halide perovskite $M A P b I_{3-x} B r_{x}$ and $M A P b I_{3-x} C l_{x}$, obtaining a significant improvement of perovskite stability $[226,235,271]$.

On the other hand, improving the efficiency of perovskite-based solar cells is also a fundamental feature. Among the different results obtained by compositional engineering, it has been found that mitigating nonradiative carrier losses could substantially improve efficiency of perovskite solar cells $[281,282]$. It has been shown that under-coordinated ions may exist at grain boundaries and surfaces that can act as charge carrier trap/recombination centers $[283,284]$. Different passivation methods have been employed, such as an iodopentafluorobenzene (IPFB) post-treatment to passivate under-coordinated iodine [282], thiophene and pyridine to passivate under-coordinated $\mathrm{Pb}$ ions [285] and recently $\mathrm{Br}$ and $\mathrm{Cl}$ to assist in suppressing recombination and decoupling electron-hole pairs [286, 288].

Recently, employing the SISSO (Sure Independence Screening and Sparsifying Operator) approach to identify an improved tolerance factor for predicting whether a given compound will be stable as perovskite, J. Bartel et al. [112] have been able to define a new tolerance factor, that allows to achieve a $92 \%$ overall accuracy. Using this new tolerance factor, the probability of inorganic and organic-inorganic double perovskite formation for thousands of unexplored compounds has been predicted, resulting in a library of around 90,000 new stable perovskites for use in various applications, including photovoltaics.

Among the cations that can form a perovskite structure, $R b, C s, M A$, and/or formamidinium $(F A)$ are the most commonly studied. Molecules larger than $F A$, such as ethylammonium $(E A)$, guanidinium $(G A)$, and imidazolium (IM) have been investigated [83], obtaining suitable candidates for multication $3 D$ perovskites [45]. 
With respect to organic guanidinium $(G A)$, a extensive research has been carried out to investigate the consequences of a $G A$ addition in $\mathrm{MAPbI}_{3}$ on structure, film quality and performance. Also, recently, it has been demonstrated a passivation effect through partial $G A$ incorporation in perovskite films, obtaining a significant mitigation of nonradiative decay and an enhanced carrier lifetime, high open circuit voltages and improved device performances [288].

The $I M$ cation presents a large ionic radius $(258 \mathrm{pm})$, suitable to form a perovskite structure. The aim of this chapter is to study the effect of partial $I M$ incorporation on $M A P b I_{3}$ perovskite thin films on optical and structural properties.

\subsection{Experimental Procedure}

\subsubsection{Perovskite Fabrication}

Single crystal of methylammonium iodide $\left(\mathrm{CH}_{3} \mathrm{NH}_{3} \mathrm{I}, \mathrm{MAI}\right)$ powder were synthesized by reacting equimolar of hydriodic acid (57\% in water, Sigma Aldrich) and methylamine $\left(\mathrm{CH}_{3} \mathrm{NH}_{2}\right)$ solution $(33 \%$ in methanol, Sigma Aldrich) with stirring during $2 h$ in a $250 \mathrm{~mL}$ round-bottom flask, which was kept in an ice bath $\left(0^{\circ} C\right)$. The precipitate was collected by placing the solution into a rotary evaporator while carefully removing the solvents at $50^{\circ} \mathrm{C}$. A recrystallization process of the raw product was carried out twice to get a much purer product, including the redissolution in absolute ethanol and the precipitation after the addition of diethyl ether.

Imidazolium iodine $\left(\mathrm{C}_{3} \mathrm{H}_{5} \mathrm{~N}_{2} I, \quad I M I\right)$ single crystal powder were synthesized dissolving 0.08 moles of imidazole (anhydrous, > 99\% Sigma Aldrich) in ethanol $96 \%$ (for analysis, PANREAC). Subsequently, it was neutralized with 0.08 mol HI (57\% in water, Sigma Aldrich) with stirring 
during $2 h$ in a $250 \mathrm{~mL}$ round-bottom flask, which was kept in an ice bath $\left(0^{\circ} \mathrm{C}\right)$. The precipitate was collected and followed the same solvent evaporation and recrystallization process previously described for $M A I$, to obtain a product as pure as possible.

The desired solutions of $M A_{1-x} I M_{x} \mathrm{PbI}_{3}(0 \leq x \leq 1)$ were prepared under an inert atmosphere in glove box by dissolution of the $M A I$, and $I M I$ powders with $\mathrm{PbI}_{2}$ (Sigma Aldrich) at $45 \%$ wt in $D M F$ (Dimethylformamide). The solutions were kept under stirring at $65^{\circ} \mathrm{C}$ for 30 minutes.

The films were deposited using the spin coating process under speed of 5000 rpm for 20 seconds, onto conductive glass substrates coated with Indium Tin Oxide (ITO). The samples of $M A P b I_{3}$ and $M A_{1-x} I M_{x} \mathrm{PbI}_{3}$ were annealed at $100^{\circ} \mathrm{C}$ for $1 \mathrm{~h}$ and $\mathrm{IMPbI}_{3}$ films were annealed during 15 minutes.

\subsection{Results and Discussion}

\subsubsection{X-ray Diffraction}

Figure 9.1 shows the results of the $X R D$ analyses for pure $M A P b I_{3}$ and mixed $M A_{1-x} I M_{x} \mathrm{PbI}_{3}$ perovskite samples. The (100) and (200) perovskite characteristic peaks can be observed at approximately $14.1^{\circ}$ and $28.4^{\circ}(2 \theta)$, respectively. As it can be seen, $\mathrm{MAPbI}_{3}$ perovskite characteristic peaks lose intensity as the quantity of $I M$ in the sample increases. Once the $I M$ content reached about $10 \%$, the $I M$ characteristic peaks began to be visible at $11.34^{\circ}$, establishing the solubility limit. This peak increased its intensity as the $I M$ content exceeded $10 \%$, reaching the value $11.27^{\circ}$ for pure $I M P b I_{3}$.

This suggests that the addition of $I M$ cation introduces a lattice distortion and eventually dominates the phase competition over $M A$ cation.

In addition, among all the studied samples under the solubility limit of $10 \%, M A_{0.94} I M_{0.06}$ thin film showed a larger intensity of the peak (100), 
indicating that a small quantity of $I M$ content can improve the crystallinity.

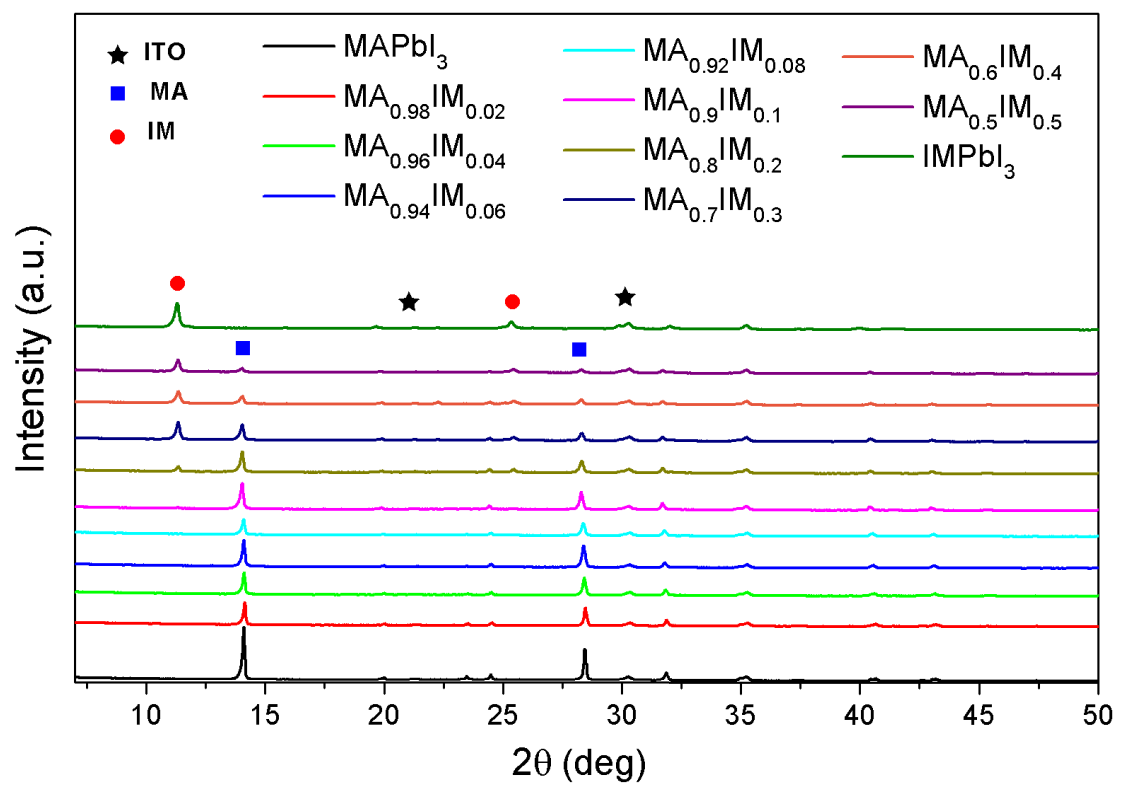

Figure 9.1: $X R D$ for $M A_{1-x} I M_{x} P b I_{3}(0 \leq x \leq 1)$.

Magnified $X$-ray diffractograms of $M A_{1-x} I M_{x} P b I_{3}$ for $(0 \leq x \leq 0.5)$ in the region of the strongest plane intensity, located in the interval $13.5^{\circ}-14.5^{\circ}$ $(2 \theta)$, are shown in Figure 9.2. The position of the peak shifts to lower angles as the average fraction of imidazolium iodide introduced in the films increases.

Film crystallinity was estimated with the Scherrer equation to determine the approximate size of crystallites, $D$, shown in equation

$$
D=\frac{K \lambda}{\beta \cos (\theta)}
$$

where $K$ was the dimensionless shape factor (0.9), $\lambda$ was the $X$-ray wavelength, and $\beta$ was the peak broadening at the $F W H M$ for the peak at $\theta$. The $X$-ray around $2 \theta=14^{\circ}$ was used for analysis, as it was distinct in all cases and free from any $\mathrm{PbI}_{2}$ overlap. It has been obtain that the grain size decreases when 
the FWHM increases, and the results are shown in Table 9.1.

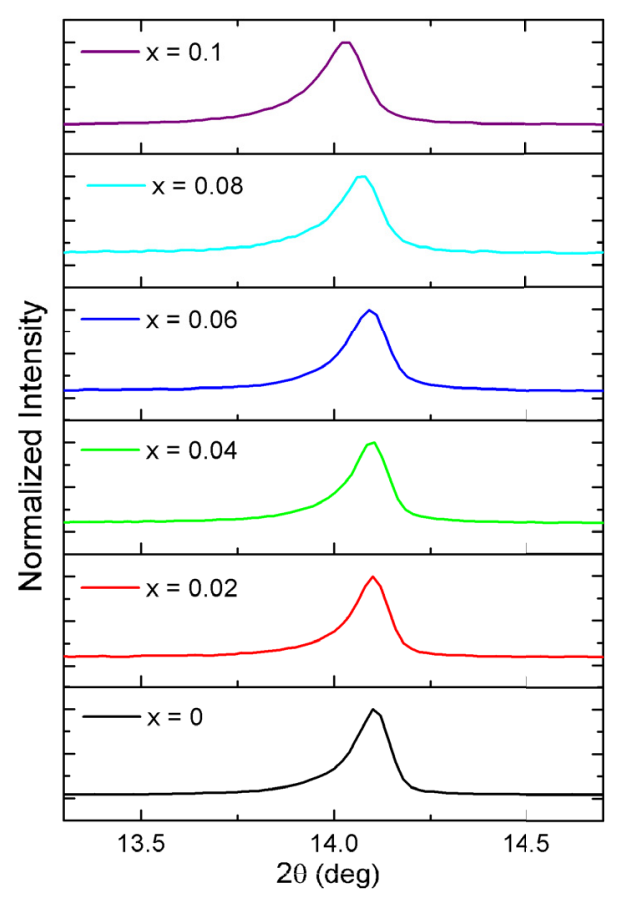

Figure 9.2: Magnified $X R D$ of $M A_{1-x} I M_{x} P b I_{3}$ for $0 \leq x \leq 0.1$, in the region of $13.5^{\circ}-14.5^{\circ}(2 \theta)$.

Table 9.1 FWHM and crystal size for $M A_{1-x} I M_{x} P b I_{3}(0 \leq x \leq 0.1)$.

\begin{tabular}{lcc}
\hline Sample & $F W H M$ & Crystal Size $(n m)$ \\
\hline$M A P b I_{3}$ & 0.1287 & $62.3 \pm 5$ \\
$M A_{0.98} I M_{0.02} \mathrm{PbI}_{3}$ & 0.1307 & $61.3 \pm 8$ \\
$M A_{0.96} I M_{0.04} \mathrm{PbI}_{3}$ & 0.1396 & $57.4 \pm 4$ \\
$M A_{0.94} \mathrm{IM}_{0.06} \mathrm{PbI}_{3}$ & 0.1511 & $53.0 \pm 6$ \\
$M A_{0.92} \mathrm{IM}_{0.08} \mathrm{PbI}_{3}$ & 0.1635 & $49.0 \pm 3$ \\
$M A_{0.9} I M_{0.1} \mathrm{PbI}_{3}$ & 0.1738 & $46.1 \pm 4$ \\
\hline
\end{tabular}

A Pawley refinement of $X R D$ spectra was carried out to determine the 
lattice parameter of $I M \mathrm{PbI}_{3}$, finding a hexagonal lattice type (space group P63mc). ITO peaks were neglected when applying Pawley refinement. In Figure 9.3 it can be observed that experimental and simulated diffractograms fit significantly well.

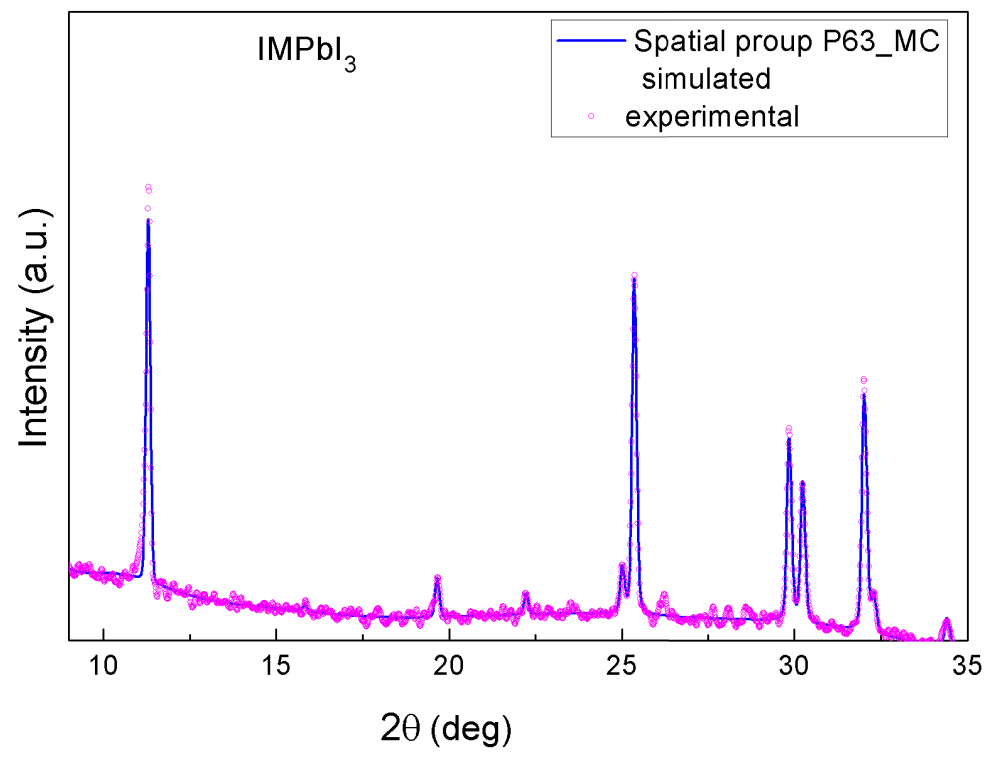

Figure 9.3: Pawley refinement of $X R D$ for pure $I M P b I_{3}$ film.

With the objective to elucidate the influence of $I M$ incorporation on the film crystallinity, a Pawley refinement of $M A_{1-x} I M_{x} P b I_{3}(x=0,0.1)$ was carried out (see Figure 9.4) obtaining a tetragonal lattice type (space group $I 4 \mathrm{~cm})$. As it can be seen, the addition of $I M$ produces a slight shift toward lower angles and a decrease of intensity of the characteristic $M A$ perovskite peak (100), located at $14.2^{\circ}$, approximately. Table 9.2 summarizes the results of the lattice parameters of $M A_{1-x} I M_{x} \mathrm{PbI}_{3}$ for $0 \leq x \leq 0.1$.

Figure 9.5 shows the values of lattice parameters as a function of $I M$ content $x$. Lattice parameter a slightly deviates from an increasing linear trend, following Vegard's law. Lattice parameter $c$ shows a decreasing trend 
when increasing the $I M$ content, with a rapid decrease when $x$ varies between 0 and 0.02. Moreover, for the values 0.02 and 0.04 , a quasi-cubic lattices are found. In agreement with the $X R D$ results, where $I M$ characteristic peaks begin to be visible when the $I M$ content exceeds $10 \%$, it can be observed an increase in the lattice parameter $c$ when $x$ content reaches this quantity. This behavior may indicate a probable lattice change from this value of $x$.

Table 9.2 Lattice parameters of $M A_{1-x} I M_{x} P b I_{3}$ for $0 \leq x \leq 0.1$

\begin{tabular}{lccc}
\hline Sample & $a=b(\AA)$ & $c(\AA)$ & $R w p$ \\
\hline$M A P b I_{3}$ & 8.86971 & 12.66157 & $6.03 \%$ \\
$M A_{0.98} I M_{0.02} \mathrm{PbI}_{3}$ & 8.87941 & 12.60061 & $5.67 \%$ \\
$M A_{0.96} I M_{0.04} \mathrm{PbI}_{3}$ & 8.88771 & 12.60402 & $5.10 \%$ \\
$M A_{0.94} I M_{0.06} \mathrm{PbI}_{3}$ & 8.91523 & 12.58795 & $5.07 \%$ \\
$M A_{0.92} I M_{0.08} \mathrm{PbI}_{3}$ & 8.91865 & 12.58288 & $4.28 \%$ \\
$M A_{0.9} I M_{0.1} \mathrm{PbI}_{3}$ & 8.91494 & 12.60558 & $5.54 \%$ \\
\hline
\end{tabular}

Furthermore, Figure 9.6 shows the dependence of the $F W H M$ and the $X R D$ peak (100) on the $I M$ content. It easy to see that, as the $I M$ content increases, the values of $F W H M$ increase and therefore, by Scherrer's equation, the grain size decreases, since $\theta$ does not vary significantly. 


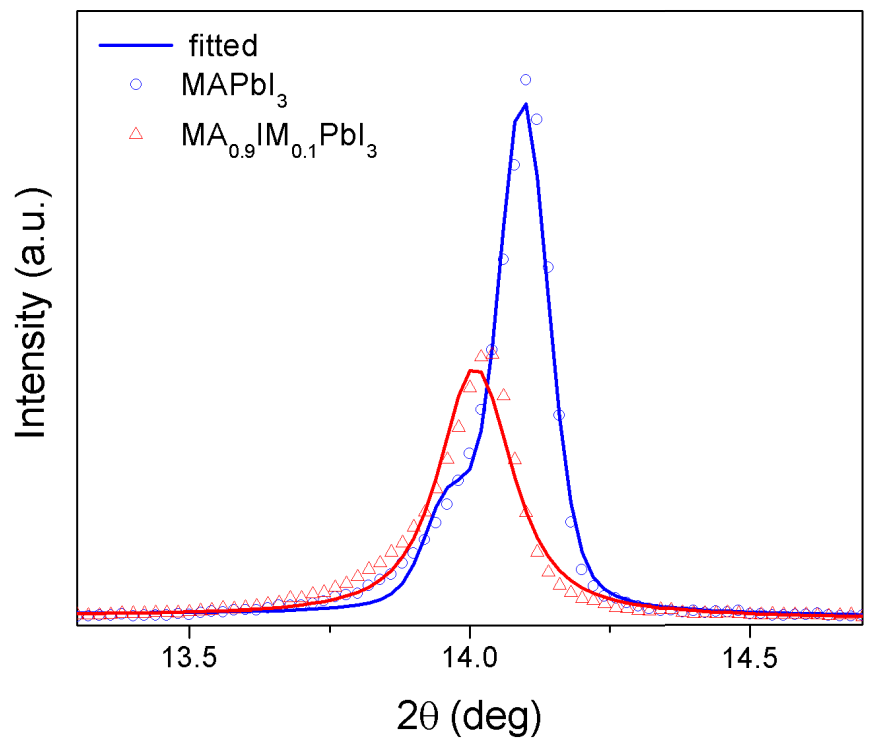

Figure 9.4: Pawley fit of $X R D$ patterns of the $M A P b I_{3}$ and $M A_{0.9} I M_{0.1} \mathrm{PbI}_{3}$.

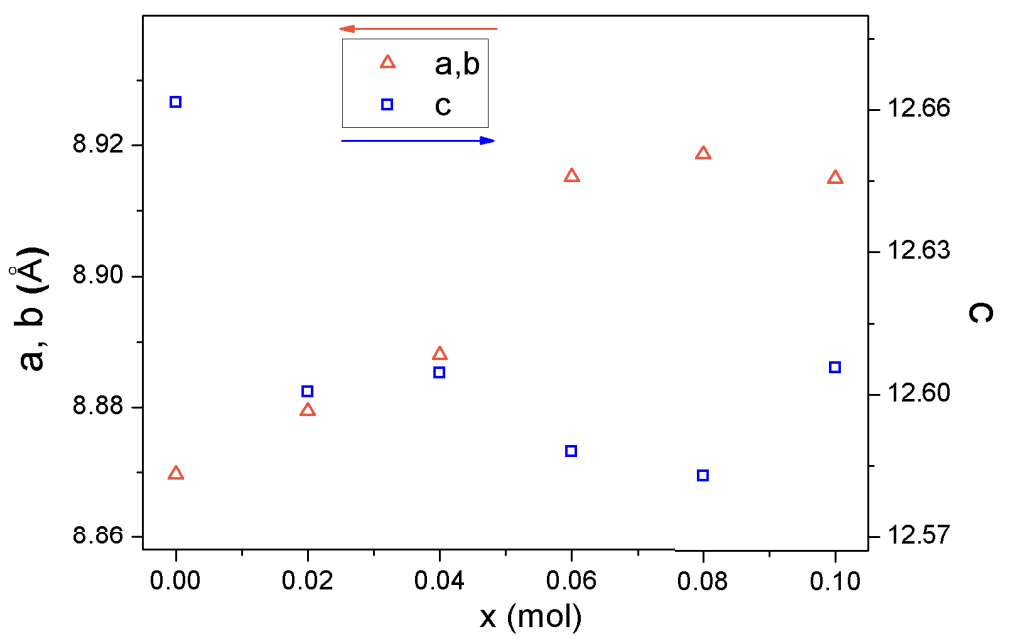

Figure 9.5: Composition-dependent evolution of lattice parameters as a function of $I M$ content. 


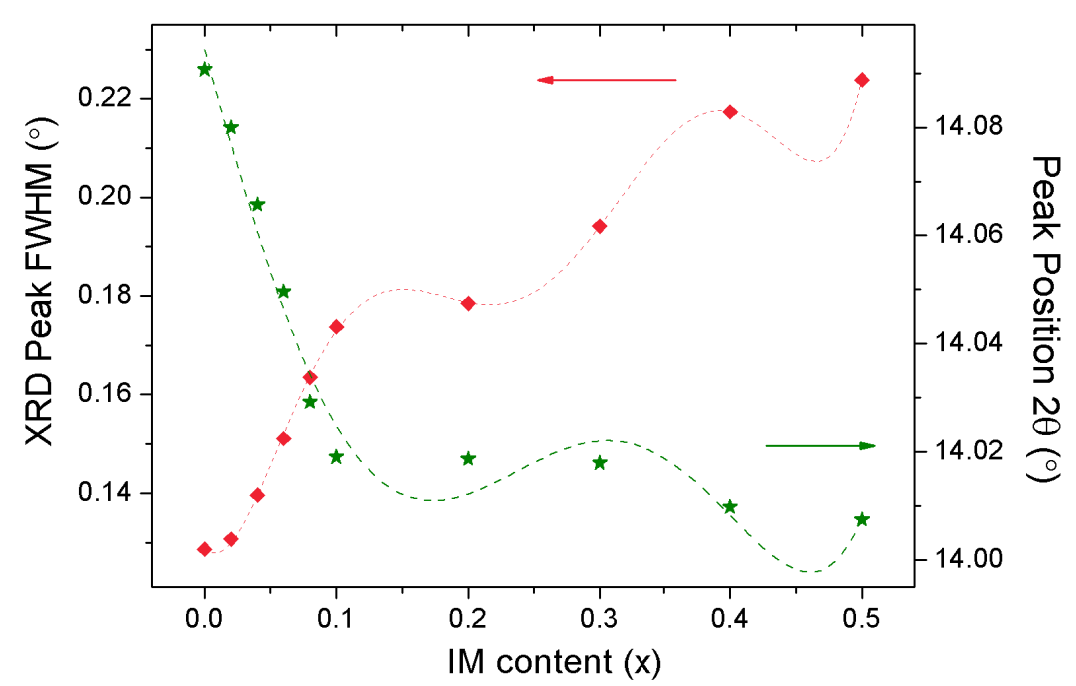

Figure 9.6: Dependence of the $F W H M$ and the $X R D$ peak (100) on the $I M$ content.

\subsubsection{Absorbance}

In order to better ascertain the influence of incorporated $I M$ cation on resulting defect density of the thin film, the absorption spectra was studied using an integrating sphere to collect both specular and diffuse transmittance to reduce the effect of light scattering originated from refraction and reflection phenomena on the perovskite crystals. Figure 9.7 presents the absorption spectra and the influence of incorporated $I M$ cation. As it can be observed, when the amount of $I M$ increases, the front edge decreases, reducing the absorbance. For $I M$ contents greater than $10 \%$, a decrease in the slope is observed, indicating a degradation of the bandgap, in agreement with the suggested lattice change mentioned before. 


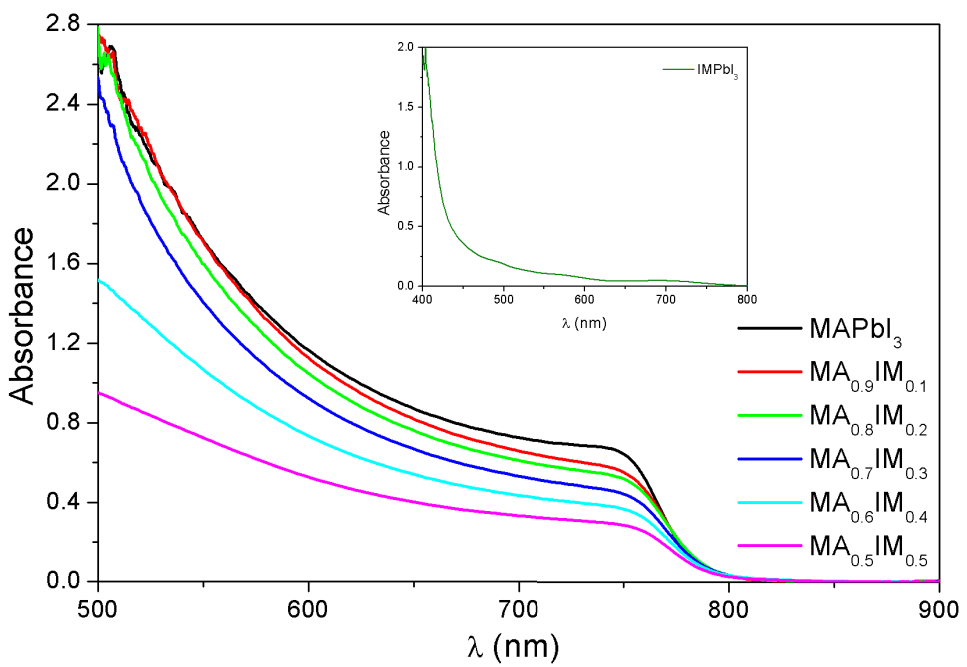

Figure 9.7: $U V$-vis absorption spectra of $M A_{1-x} I M_{x} P b I_{3}$ for different $I M$ contents.

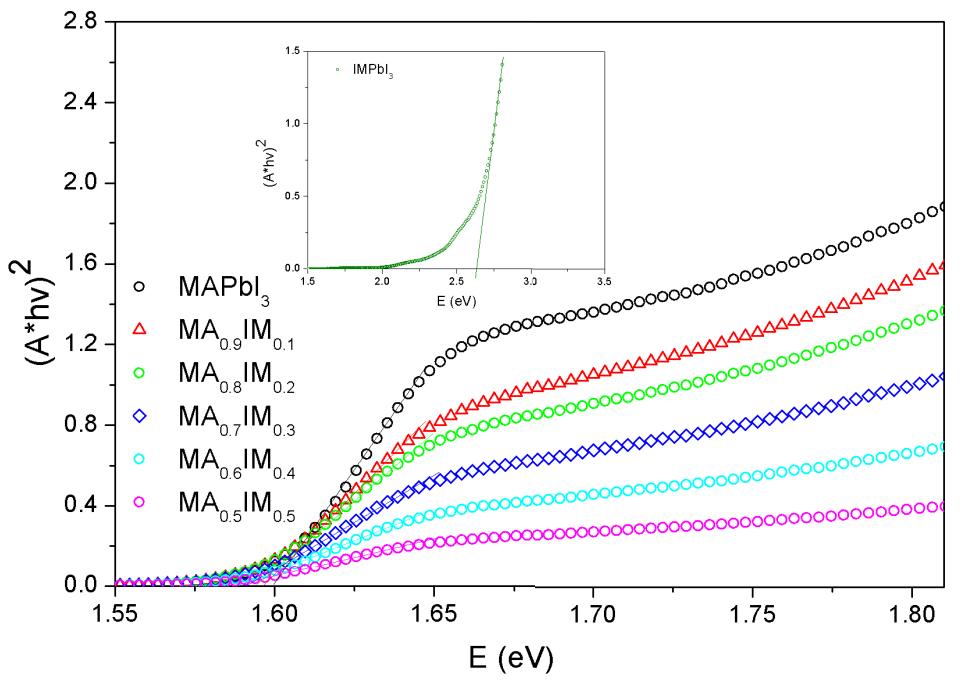

Figure 9.8: Tauc plots of $M A_{1-x} I M_{x} \mathrm{PbI}_{3}$ showing variations in optical bandgap.

The variation in the optical bandgap, shown in the Tauc plots is provided in Figure 9.8. For the samples with a $I M$ content between $0 \leq x \leq 0.5$, the bandgap changes slightly towards lower energies. The inset of the 
Figure 9.8 display the estimated bandgap for $I M P b I_{3}$ sample $(2.64 \mathrm{eV})$ corresponding to an absorption edge of $469 \mathrm{~nm}$. The estimated bandgaps $\left(E_{g}\right)$ for $M A_{1-x} I M_{x} \mathrm{PbI}_{3}$ for $0 \leq x \leq 0.5$ are shown in Table 9.3.

Table 9.3 Bandgap variation depending on $I M$ content.

\begin{tabular}{lcc}
\hline Sample & \multicolumn{2}{c}{ Absorption edge at room T } \\
\cline { 2 - 3 } & $\mathrm{eV}$ & $\mathrm{nm}$ \\
\hline $\mathrm{MAPbI}_{3}$ & 1.6 & 775.0 \\
$\mathrm{MA}_{0.9} I M_{0.1} \mathrm{PbI}_{3}$ & 1.59 & 779.9 \\
$\mathrm{MA}_{0.8} I M_{0.2} \mathrm{PbI}_{3}$ & 1.59 & 779.9 \\
$\mathrm{MA}_{0.7} \mathrm{IM}_{0.3} \mathrm{PbI}_{3}$ & 1.59 & 779.9 \\
$M A_{0.6} \mathrm{IM}_{0.4} \mathrm{PbI}_{3}$ & 1.58 & 784.8 \\
$M A_{0.5} I M_{0.5} \mathrm{PbI}_{3}$ & 1.58 & 784.8 \\
$\mathrm{IMPbI}$ & 2.64 & 470.0 \\
\hline
\end{tabular}

It can be seen that the bandgap remains almost constant, the overall variation between all the samples being from 1.6 to $1.58 \mathrm{eV}$, corresponding to absorption edges spanning from 775 to $784.8 \mathrm{~nm}$.

\subsubsection{Photoluminescence}

The $P L$ spectrum intensity of $M A_{0.8} I M_{0.2} \mathrm{PbI}_{3}$ film is three times higher than that observed for $\mathrm{MAPbI}_{3}$, suggesting that nonradiative recombination has been significantly reduced. Furthermore, as it can be seen in Figure 9.9, the $P L$ peak for $M A_{0.8} I M_{0.2} P b I_{3}$, centered at $738 \mathrm{~nm}$, is shifted towards higher energies when compared with the $P L$ peak of $\mathrm{MAPbI}_{3}$, centered at $770 \mathrm{~nm}$. This implies that the incorporation of $I M$ cation inhibits nonradiative charge recombination and then act as an effective passivator. 


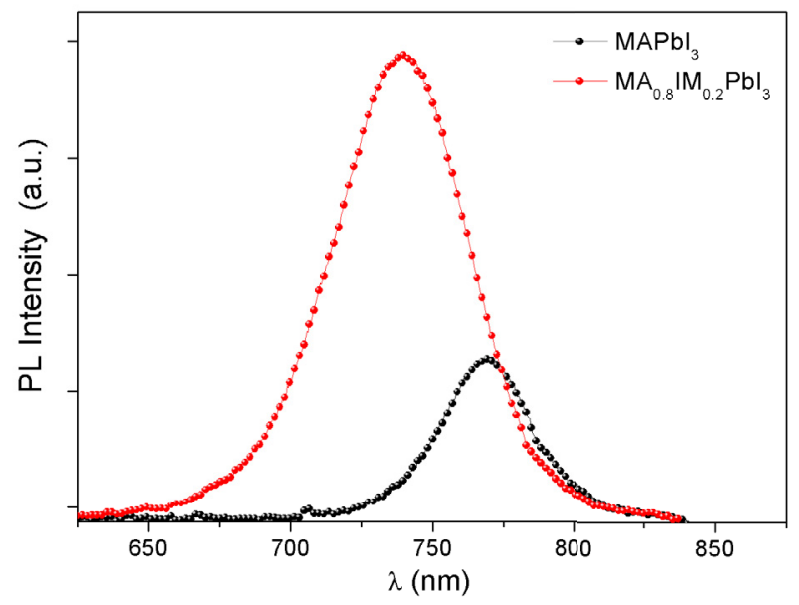

Figure 9.9: $P L$ spectra of $M A_{1-x} I M_{x} P b I_{3}$ for $x=0,0.2$.

The absorption and $P L$ spectra of pure $M A P b I_{3}$ and mixed $M A_{0.8} I M_{0.2} \mathrm{PbI}_{3}$ are given in Figures 9.10 and 9.11. $M A P b I_{3}$ presents an absorption edge at $775 \mathrm{~nm}$, very similar to that observed for $M A_{0.8} I_{0.2} \mathrm{PbI}_{3}$ $(779.8 \mathrm{~nm})$, both emission are located in the limit of vis-IR region. This short difference in the absorption edge does not justify the shift observed in the $P L$ spectra. That means that an additional mechanism should be involved to explain the blue shift in the $P L$ emission of $M A_{0.8} I M_{0.2} \mathrm{PbI}_{3}$ with respect to the $M A P b I_{3}$. Some authors explain this behavior through the existence of vibronic relaxation [254]. The $P L$ spectra were well fitted by means of a Gaussian function, obtaining a $F W H M$ of about $40.73 \mathrm{~nm}$ and $57.58 \mathrm{~nm}$ for $M A P I$ and $M A_{0.8} I_{0.2} \mathrm{PbI}_{3}$, respectively. Narrower $F W H M$ means narrower bandwidth in the initial or/and final states involved in the $P L$, which can also be related with fewer defects, such as impurities, pinholes, grain boundaries, etc. 


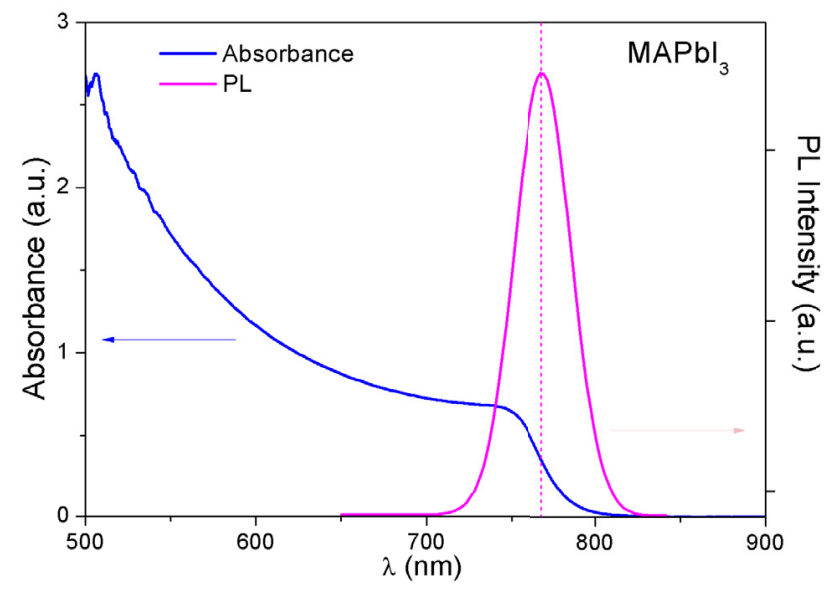

Figure 9.10: $P L$ spectrum and absorbance of $M A P b I_{3}$ at room temperature.

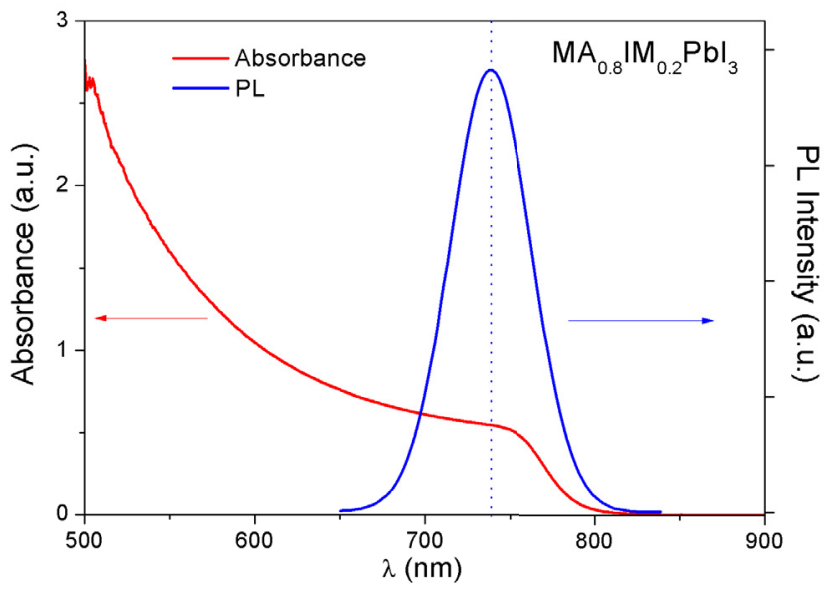

Figure 9.11: $P L$ spectrum and absorbance of $M A_{0.8} I M_{0.2} \mathrm{PbI}_{3}$ at room temperature.

\subsubsection{FESEM analysis}

The morphology variation of the studied $M A_{1-x} I M_{x} P b I_{3}$ films was examined using FESEM. The $\mathrm{MAPbI}_{3}$ film demonstrated a decent crystallinity with an average grain size of about $130 \mathrm{~nm}$ (see Figure 9.12a). However, some pinholes could be observed on the film surface, which has been 
well documented to incur in short circuit, thereby impairing the derived $P V$ performance. In contrast, the $M A_{0.94} I M_{0.06} \mathrm{PbI}_{3}$ thin film presents a more ideal morphology with an almost pinhole-free surface. Moreover, it consists of grain domains with a medium grain size of $100 \mathrm{~nm}$ as shown in Figure 9.12b. The $M A_{0.8} I M_{0.2} \mathrm{PbI}_{3}$ film shows a pin-hole free compact surface of an average grain of $50 \mathrm{~nm}$, creating a larger number of grain boundaries that can act as charge carrier trap/recombination centers (see Figure 9.12c).
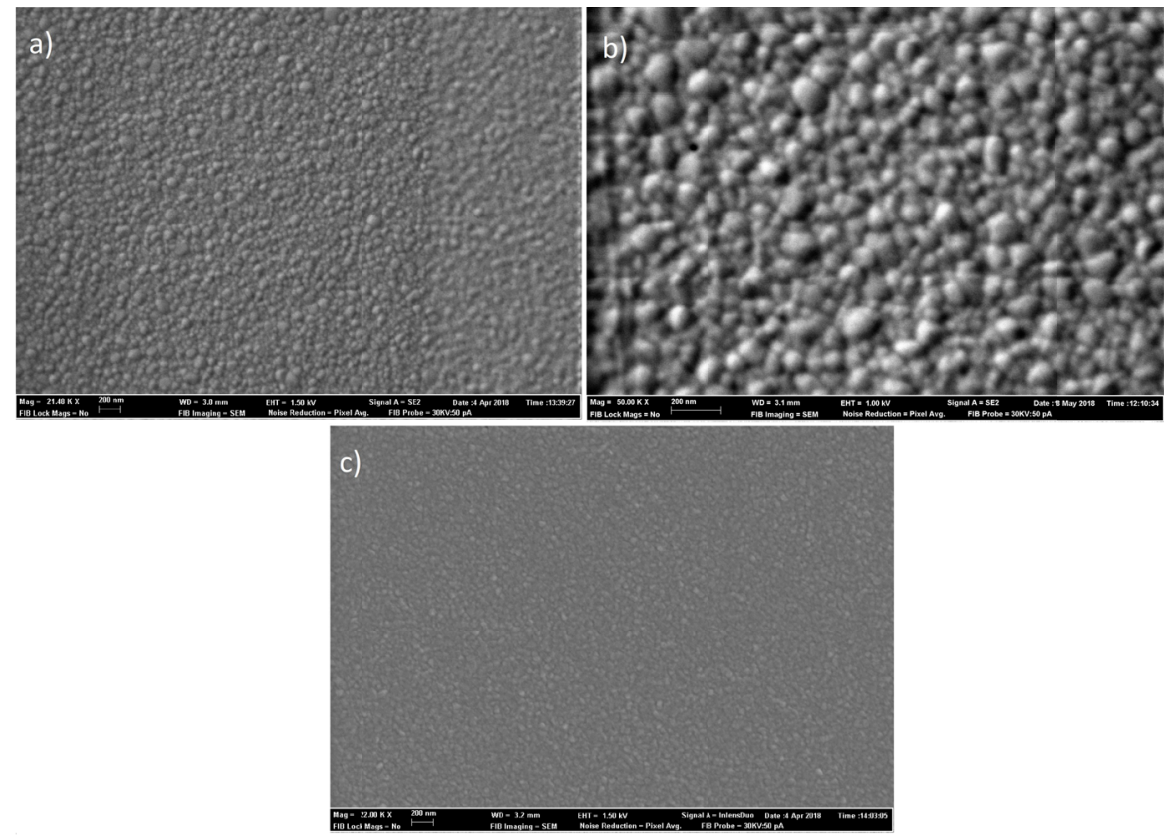

Figure 9.12: FESEM micrographs of (a) $\mathrm{MAPbI}_{3}$, (b) $M A_{0.94} \mathrm{IM}_{0.06} \mathrm{PbI}_{3}$ and (c) $M A_{0.8} I M_{0.2} \mathrm{PbI}_{3}$ thin films .

\subsection{Conclusions}

In this chapter, we studied the effect of partial $I M$ incorporation on $\mathrm{MAPbI}_{3}$ perovskite thin films on optical and structural properties. $X$-ray diffraction analyses have established the limit of solubility of $I M$ at about 
10\%. Mixed perovskites with an $I M$ content below $10 \%$, and specifically the $M A_{0.94} I M_{0.06} \mathrm{PbI}_{3}$ thin film, showed a larger intensity of the peak (100), indicating an improvement in crystallinity.

Film crystallinity was estimated with the Scherrer equation, indicating that the grain size decreases when the $F W H M$ increases. A Pawley refinement of $M A_{1-x} I M_{x} \mathrm{PbI}_{3}(x=0,0.1)$ was carried out obtaining a tetragonal $I 4 \mathrm{~cm}$ lattice type. Also, lattice parameter $a=b$ increased linearly as the $I M$ content increased, contrary to the behavior of lattice parameter $c$, that decreased until the $I M$ content reached $10 \%$, indicating a probable lattice change when $x$ exceeds this value.

Absorption spectra showed a decrease in the absorption front edge when the $I M$ content increased up to $10 \%$. For $I M$ contents above $10 \%$, a degradation of the bandgap could be appreciated, in agreement with the suggested lattice change.

The $P L$ spectrum of $M A_{0.8} I M_{0.2} \mathrm{PbI}_{3}$ film shifted towards higher energies and showed an intensity three times higher when compared with the $M A P b I_{3}$ spectrum, suggesting that $I M$ incorporation reduces nonradiative recombination significantly and acts as an effective passivator. This result is in agreement with the data in the literature for mixed $M A$ - $I M$-based perovskite thin films.

Finally, FESEM analysis showed an improved morphology for the mixed $M A_{1-x} I M_{x} P b I_{3}$ thin films, when compared with that of $M A P b I_{3}$. Specifically, the $M A_{0.94} I M_{0.06} \mathrm{PbI}_{3}$ thin film presented a pinhole-free uniform and smooth morphology with grain sizes of about $100 \mathrm{~nm}$. However, the $M A_{0.8} I M_{0.2} \mathrm{PbI}_{3}$ film showed a smaller grain size of about $50 \mathrm{~nm}$ and a larger number of grain boundaries able to act as charge carrier trap/recombination centers. 


\section{Chapter 10}

\section{Conditions for Fabricating}

\section{Efficient Devices with}

\section{Methylammonium Lead \\ Halide Perovskite}

\subsection{Motivation}

The methylammonium lead halide perovskite $M A P b I_{3}$ and its mixed halide crystals have shown numerous optimal optoelectronic features of an ideal solar cell absorber, such as a favorable bandgap, high absorption coefficient, excellent charge mobility, high photoluminescence quantum efficiencies, small exciton binding energy, tunable bandgap and a reasonable defect tolerance. One of the key features of a perovskite-based device is its power efficiency conversion $(P C E)$, commonly used to compare the performance of different solar devices. In the last decade, the $P C E$ of perovskite-based solar devices has increased from $3.9 \%$ in 2009 [46] to a 
certified $22.7 \%$ in 2017 [19], close to that of $S i$-based devices.

In the synthesis, deposition and fabrication of solar devices there are numerous factors that can affect their performance. Extensive research has been conducted worldwide with the aim of improving the efficiency of the devices. Good results have been obtained in compositional engineering, studying the substitution of both the cation and the anion in the perovskite structure by suitable candidates. Some of these substitutions are investigated and reviewed in this thesis.

Improving the experimental methods (synthesis, deposition and post-treatments) has been proved as an efficient way to enhance performance. In particular, the mitigation of the influence of moisture has been a key feature for various perovskite fabrication methods. Recently, several special methods for fabricating high-efficiency perovskite solar cells in ambient air have been reported, such as one-step spin-coating methods [291]. These methods have demonstrated that the film coverage at the spin-coating stage can be improved through careful control of the moisture.

The use of anti-solvents during the spin-coating process has led to very good results concerning homogeneity and grain size of the thin films, and therefore, to improved device efficiencies [166, 174-178].

Recently, a criterion has been formulated for the formation of high performance devices under environmental conditions, establishing that the $\mathrm{H}_{2} \mathrm{O} / \mathrm{DMSO}$ ratio needs to be carefully adjusted to obtain adequate morphology and to reduce the presence of chemical defects [290]. It has been proved that both molecules compete to coordinate with the $\mathrm{Pb}$ atom, which will limit the formation of the intermediate complex $\mathrm{PbI}_{2}: \mathrm{MAI}$ :additive responsible for the final properties of the films.

In this chapter, a device fabrication process for $M A P b I_{3}$ is presented, 
employing diethyl ether as anti-solvent during the spin-coating stage, taking into account relative humidity conditions and spiro-OMeTAD oxidation. $P L$ and FESEM analyses are presented, together with $J-V$ measurements of the devices showing the highest efficiencies (between $11 \%$ and $15 \%$ ).

\subsection{Experimental Procedure}

\subsubsection{Materials and Precursor Solutions}

All materials were used as received: FTO substrates $(2.5 \times 2.5$ cm, Pilkintong TEC15, $15 \Omega s q-1$ resistance), titanium diisopropoxide bis (acetylacetonate) (75\% in isopropanol, Sigma Aldrich), $\mathrm{TiO}_{2}$ paste (30NR-D, $300 \mathrm{~nm}$ average particle size, Greatcell solar), $\mathrm{CH}_{3} \mathrm{NH}_{3} \mathrm{I}$ (Greatcell solar), $\mathrm{PbI}_{2}$ (99.99\%, TCI) and spiro-OMeTAD (Merck).

\subsubsection{Photovoltaic Devices Fabrication}

All devices were prepared over FTO substrates $(2.5 \times 2.5 \mathrm{~cm})$, partially etched with zinc powder and $\mathrm{HCl}(2 M)$ in order to avoid short circuits, obtaining $0.25 \mathrm{~cm}^{2}$ of active electrode area. Then, the substrates were washed through ultrasonication for $15 \mathrm{~min}$, first by soap (2\%, Hellmanex) and rinsed with deionized water, subsequently the process it was repeated with ethanol, acetone and finally with isopropanol. After that, the substrates were dried with compressed air and treated in a $U V-O$ chamber for 15 minutes.

The $\mathrm{TiO}_{2}$ blocking layer was deposited onto substrates by spray pyrolysis at $450^{\circ} \mathrm{C}$, using a titanium diisopropoxide bis (acetylacetonate) solution diluted in ethanol $(1: 9, v / v)$ with oxygen as the carrier gas. Spray pyrolysis was performed in three steps with $5 \mathrm{~mL}$ of the solution, spraying each time $6 s$, and waiting for $30 s$ between steps. When this process was finished, the films were kept at $450^{\circ} \mathrm{C}$ for $30 \mathrm{~min}$. 
The mesoporous $\mathrm{TiO}_{2}$ layer was prepared using a $\mathrm{TiO}_{2}$ paste diluted in ethanol (1:5, weight ratio) and deposited by spin coating at $2000 \mathrm{rpm}$ for $10 \mathrm{~s}$. After drying at $100^{\circ} \mathrm{C}$ for $10 \mathrm{~min}$, the mesoporous layer was heated at $500^{\circ} \mathrm{C}$ for $30 \mathrm{~min}$ and then cooled to room temperature.

The perovskite precursor solution was prepared by mixing $D M F$ solutions (50 wt \%) containing $M A I, P b I_{2}$ and $D M S O$ (1:1:1 mol \%). First, $\mathrm{PbI}_{2}$ must be dissolved in the solvent mixture (DMF:DMSO) at $65^{\circ} \mathrm{C}$ without stirring and then cooled down until room temperature to mix with $M A I$. Subsequently, $50 \mu L$ of this solution was spin-coated at $4000 \mathrm{rpm}$ for 50 s. $D M F$ was selectively washed with non-polar diethyl ether while one-step spin-coating process was carried out. The diethyl ether has to be added just before the white solid begins to crystallize in the substrate. Under ambient conditions the time of adding the diethyl ether varies depending on the relative humidity. At 30\% R.H. the diethyl ether must to be added 8-9 $s$ after the spin begins, and at $60 \%$ R.H. after 12-13 s. Immediately after perovskite deposition, the substrates were annealed at $100^{\circ} \mathrm{C}$ for $3 \mathrm{~min}$.

Later, the perovskite films were covered with the hole transport material $(H T M)$ by dynamically spin coating at $4000 \mathrm{rpm}$ for $30 \mathrm{~s}$, using $50 \mu L$ of spiro-OMeTAD solution. The spiro-OMetAD solution was prepared by dissolving in $1 \mathrm{~mL}$ of chlorobenzene $72.3 \mathrm{mg}$ of (2,2'7,7'-tetrakis(N,N'-di-p-methoxyphenylamine)-9,9'-spirobifluorene), $\quad 28.8$ $\mu L$ of 4-tert-butylpyridine, and $17.5 \mu \mathrm{L}$ of a stock solution of $520 \mathrm{mg} \mathrm{mL}^{-1}$ of lithium bis-(tri-fluoromethylsulfonyl) imide in acetonitrile. Spiro-OMeTAD must be oxygenated for, at least, $4 h$ inside the dry-humidity controlled box, before gold evaporation, to achieve reproducibility and high efficiencies.

Finally, $60 \mathrm{~nm}$ of gold was thermally evaporated on top of the devices to form the electrode contacts using a commercial Univex 250 chamber, from 
Oerlikon Leybold Vacuum.

Photovoltaic devices were characterized using an Abbet Solar simulator equipped with a $1.5 \mathrm{AM}$ filter. The light intensity was adjusted to $100 \mathrm{Mw}$ $\mathrm{cm}^{-2}$ using a calibrated $S i$ solar cell. Devices were measured using a mask to define an active area of $0.11 \mathrm{~cm}^{2}$.

\subsection{Results and Discussion}

\subsubsection{UV-vis Spectra and Photoluminescence}

The absorption and $P L$ spectrum of pure $\mathrm{MAPbI}_{3}$ is shown in Figure 10.1. The $P L$ peak of $M A P b I_{3}$ is centered at $773 \mathrm{~nm}$ corresponding to 1.60 $e V$, coinciding approximately, with the absorption edge.

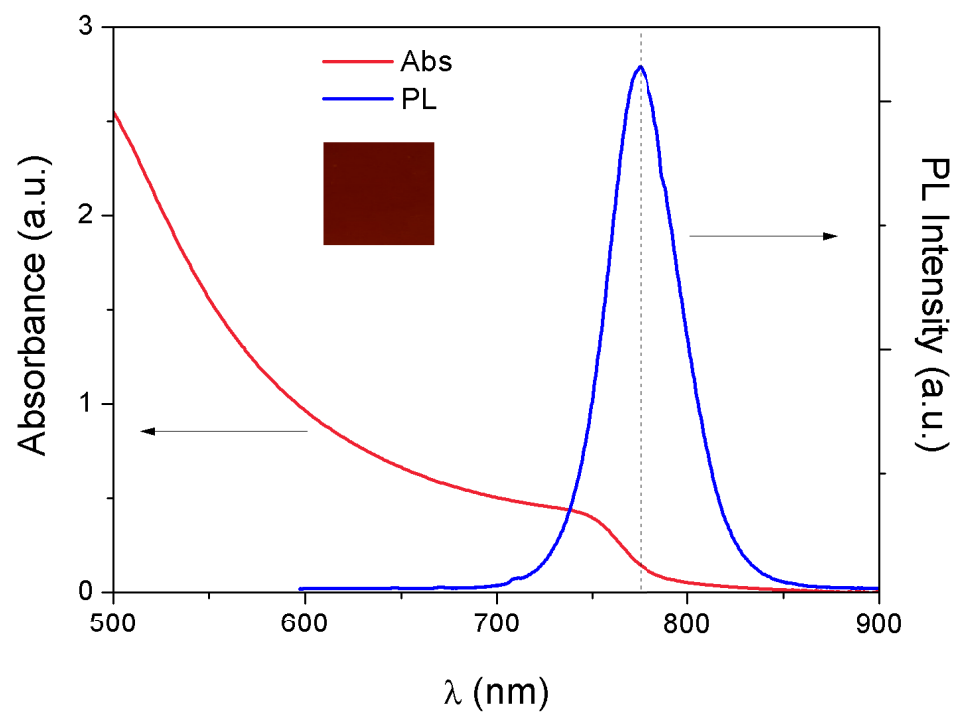

Figure 10.1: $P L$ spectrum and absorbance of $M A P b I_{3}$ thin film at room temperature. An image of the $\mathrm{MAPbI}_{3}$ thin film onto FTO is shown.

This result was obtained from a $\mathrm{MAPbI}_{3}$ thin film deposited onto FTO, showing good morphology and crystallization, and synthesized exactly as the 
thin films for device fabrication. Crystallization was carried out with diethyl ether, obtaining a homogeneous layer of bright amber color. The $P L$ spectrum showed a slight shift to the red with respect to the excitonic absorption peak, mainly caused by self-absorption due to the finite thickness of the film.

\subsubsection{FESEM analysis}

The quality and thickness of the fabricated compact layers and final device architecture were investigated by FESEM analysis. The FESEM image shows the cross section of a $\mathrm{MAPbI}_{3}$ device.

The cross-section FESEM image (Figure 10.2) shows a well-defined layered structure with clear interfaces, comprising the glass/FTO substrate, compact $\mathrm{TiO}_{2}$, mesoporous $\mathrm{TiO}_{2}, \mathrm{MAPbI}_{3}$ perovskite, hole transport material $(H T M)$, and back contact $(A u)$ layers, with approximate thicknesses of 60, 250, 220, 250 and $60 \mathrm{~nm}$, respectively. The FESEM image reveals a pinhole-free uniform perovskite film, and, the aforementioned thickness of about 220 is in agreement with the data in the literature. Furthermore, this thickness has been proved to be sufficient to serve as a light-absorbing layer.
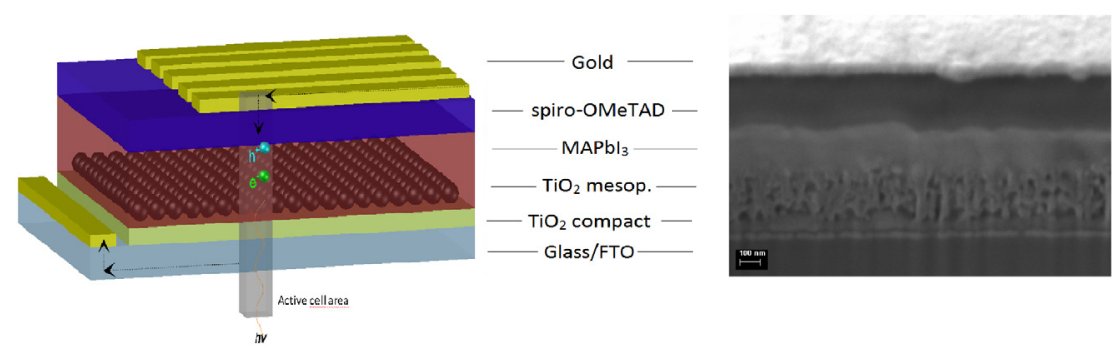

Figure 10.2: Cross-section FESEM image of the device and a device structure picture. 


\subsubsection{Current-Voltage Measurements}

In this section, the results of 8 planar devices, with high efficiencies and reproducibility are presented, each of them consisting of 5 separated pixels. This resulted in a total of 40 solar cells, having employed the same procedure, at the same time, and under the same conditions. Pixels are referred to by the cell name followed by the pixel number, e.g. $A 1-1$ refers to the first pixel of cell $A 1$. The pixel active area, defined by the overlap of the gold top electrode and the underlying FTO (non-etched area), showed a thickness of about 840 $n m$, as measured by FESEM.

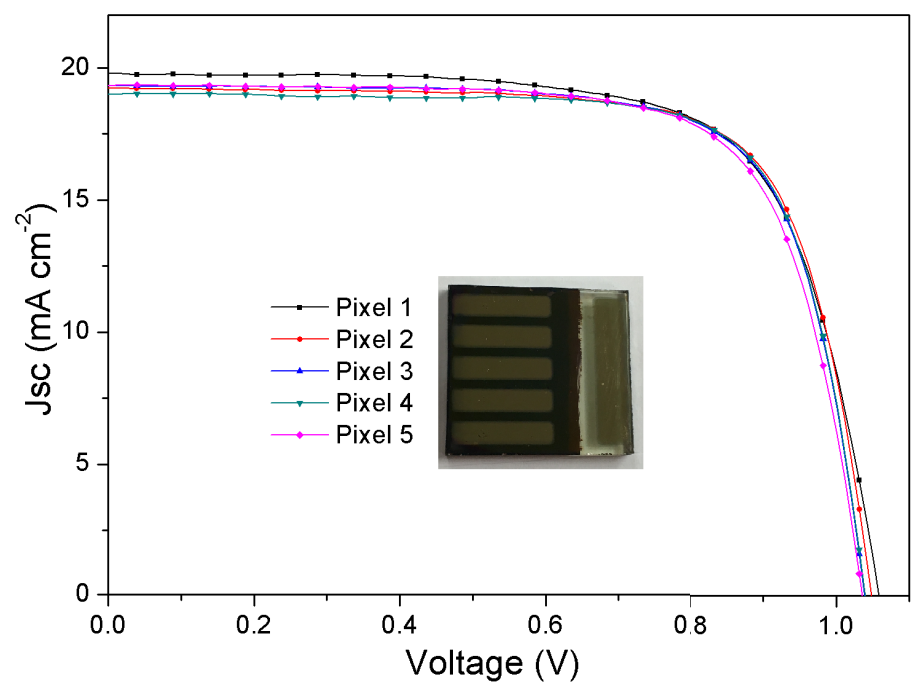

Figure 10.3: $J-V$ curve corresponding to the $A 8$ device. An actual picture of the device is shown in the inset.

The device efficiencies were measured under a solar simulator. The $J-V$ curves were measured after the gold evaporation, within the first 24 hours. For this measurement, the cells were masked using a metal aperture with an area of $0.11 \mathrm{~cm}^{2}$. These cells were measured in forward from $-0.2 \mathrm{~V}$ to short-circuit $(1.2 \mathrm{~V})$ at a rate of $10 \mathrm{mV} / \mathrm{s}$. The level of hysteresis in these devices was not 
studied. The cells had an average efficiency of $12.83 \%$ under 1 Sun $A M 1.5$ illumination.

Figure 10.3 shows the $J-V$ curve of the best device of the whole batch, and Table 10.1 shows the performance parameters corresponding to this device, labeled $A 8$, and its 5 pixels. The maximum efficiency of $14.79 \%$ was reached for pixel $A 8-2$. Moreover, each pixel reached an approximate efficiency, demonstrating a high reproducibility of the device.

Table 10.1 Performance parameters of the $A 8$ device and its 5 pixels.

\begin{tabular}{ccccccc}
\hline Device & Size & $J_{S C}$ & $I_{S C}$ & $V_{O C}$ & $F F$ & $\eta$ \\
\hline & {$\left[\mathrm{cm}^{2}\right]$} & {$\left[\mathrm{mA} / \mathrm{cm}^{2}\right]$} & {$[\mathrm{mA}]$} & {$[\mathrm{mV}]$} & {$[\%]$} & {$[\%]$} \\
\cline { 2 - 6 }$A 8 \_1$ & 0.11 & 19.79 & 2.17 & 1.05 & 70.36 & 14.73 \\
$A 8 \_2$ & 0.109 & 19.23 & 2.09 & 1.04 & 73.41 & 14.79 \\
$A 8 \_3$ & 0.109 & 19.32 & 2.10 & 1.03 & 73.23 & 14.69 \\
$A 8 \_4$ & 0.11 & 19.01 & 2.09 & 1.03 & 74.77 & 14.77 \\
$A 8 \_5$ & 0.11 & 19.34 & 2.12 & 1.03 & 72.35 & 14.49 \\
\hline
\end{tabular}

The average performance parameters corresponding to all the devices studied are shown Table 10.2.

It has been proved that oxidized spiro-OMeTAD can enhance the conductivity of the HTM layer and the charge transfer at the $A u /$ spiro-OMeTAD interface. In Figure 10.4 the $J-V$ curves of a $M \mathrm{APbI}_{3}$-based device, before and after spiro-OMeTAD oxidation, are shown. As can be seen, the performance parameters increased (inset of the graph) after the oxidation process under controlled humidity conditions (dry-humidity controlled box) for $24 \mathrm{~h}$. Particularly, the efficiency increased by $1 \%$, highlighting the importance of the oxidation process in the performance of the device. 
Table 10.2 Averages of the performance parameters of each of the 8 devices.

\begin{tabular}{ccccccc}
\hline Device & Size & $J_{S C}$ & $I_{S C}$ & $V_{O C}$ & $F F$ & $\eta$ \\
\hline & {$\left[\mathrm{cm}^{2}\right]$} & {$\left[m A / \mathrm{cm}^{2}\right]$} & {$[m A]$} & {$[m V]$} & {$[\%]$} & {$[\%]$} \\
\cline { 2 - 6 } $\mathrm{A} 1$ & 0.11 & 19.08 & 2.09 & 1.03 & 70.92 & 13.94 \\
$\mathrm{~A} 2$ & 0.11 & 15.93 & 1.75 & 1.01 & 66.05 & 10.71 \\
$\mathrm{~A} 3$ & 0.11 & 17.88 & 1.96 & 1.00 & 68.45 & 12.36 \\
$\mathrm{~A} 4$ & 0.11 & 19.08 & 2.09 & 1.02 & 71.10 & 13.93 \\
$\mathrm{~A} 5$ & 0.11 & 19.26 & 2.11 & 1.03 & 69.01 & 13.71 \\
$\mathrm{~A} 6$ & 0.11 & 18.12 & 1.99 & 0.92 & 60.20 & 10.14 \\
$\mathrm{~A} 7$ & 0.11 & 18.30 & 2.01 & 1.01 & 70.58 & 13.15 \\
$\mathrm{~A} 8$ & 0.11 & 19.34 & 2.12 & 1.04 & 72.83 & 14.70 \\
\hline
\end{tabular}

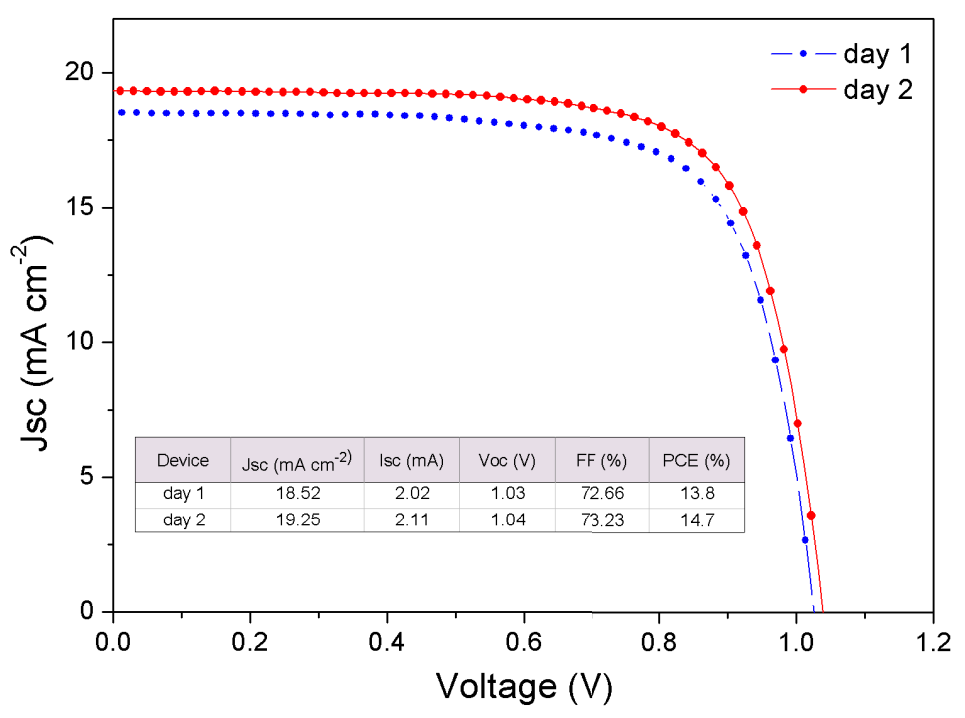

Figure 10.4: $J-V$ curve corresponding to the $A 8$ device before and after spiro-OMeTAD oxidation. 


\subsection{Conclusions}

In this chapter, the fabrication of perovskite solar cells showing a good performance, using the one-step spin-coating process, in ambient air with humidity of about $35 \%$, is reported. An efficiency of $14.79 \%$ for $\mathrm{MAPbI}_{3}$ solar cells has been reached, employing this method. A comparison of the performance parameters, before and after spiro-OMeTAD oxidation for 24 $h$ has been reported, indicating that oxidation of spiro-OMeTAD, under controlled humidity conditions, can significantly improve the performance parameters.

Each pixel on the devices showed similar efficiencies, demonstrating a high reproducibility. The crystallization with diethyl ether was carried out successfully, obtaining a homogeneous layer of bright amber color.

The cross-section FESEM image showed a well-defined layered structure with clear interfaces, a pinhole-free uniform perovskite film, and a thickness in agreement with the data in the literature.

These results make the one-step spin-coating process in air a cost-effective competitive candidate for high efficiency solar cell fabrication, when compared to other vacuum manufacturing methods. 


\section{Chapter 11}

\section{Conclusions}

In this thesis, compositional engineering has been applied with the aim to enhance optoelectronic and morphological properties of perovskite solar cells, by substituting and/or combining suitable cations and anions of the perovskite materials. The general results are summarized below:

First: since the substitution of iodine by chloride or bromide atoms is able to improve perovskite morphology and stability, we focused on the study of optoelectronic and morphological properties of methylammonium lead halide perovskites with different halides (iodide, bromide and chloride) and their mixtures, $M A P b X_{3-x} Y_{x}$ for $X, Y=I, B r, C l$ and $0 \leq x \leq 3$, in order to optimize the chemical composition and to meet the requirements for solar cell applications. $X$-ray diffractograms showed that $M A P b X_{3-x} Y_{x}$ films, for $X, Y=I, B r, C l$ and $0 \leq x \leq 3$, crystallized in cubic phase irrespective of the $X$ component and $x$ content, but the position of the $X R D$ peaks shifted according to the halide component and content in the films. Interestingly, it was found that when combining $I$ and $C l$ in perovskite mixed halides, the chloride content was so low that it should be considered a dopant agent rather than a mixture. We were able to prove that the absorption edge and bandgap 
of mixed thin film perovskites could be tuned along the entire visible spectrum.

Second: the $\mathrm{MAPbI}_{3}$ perovskite is more sensitive to moisture and humidity than $\mathrm{MAPbBr}_{3}$, however, the absorption of $M A P b B r_{3}$ is not comparable to that of $\mathrm{MAPbI}_{3}$. For this reason, we focused on the preparation of mixed halide perovskite $M A P b I_{3-x} B r_{x}$ with the aim of balancing the good properties of both compounds. In order to better understand how to optimize the chemical composition to meet the requirements of different solar applications, we carried out an investigation on the processed solutions, $X$-ray structural analyses and optical properties. $X$-ray diffraction analysis revealed the formation of a tetragonal phase $I 4 / \mathrm{mcm}$ up to a bromide content of $x=0.4$ and a cubic perovskite with space group $\operatorname{P} m \overline{3} m$ when $0.6 \leq x \leq 1$. On the other hand, absorbance measurements showed that the perovskite films exhibit a very high absorbance in the visible and short infrared region. As the fraction of bromide changes, the absorption edge of the thin films can be tuned in the visible spectrum. The incorporation of bromide into $M A P b I_{3-x} B r_{x}$ shifts the $P L$ emissions to shorter wavelengths, being located in intermediate values between $781 \mathrm{~nm}\left(\mathrm{MAPbI}_{3}\right)$ and $545 \mathrm{~nm}\left(\mathrm{MAPbBr}_{3}\right)$, implying that the defect centers come from the presence of the bromide and iodide in the perovskite lattice.

Third: following this line of research, we studied the mix of the three halides in the $M A P b I_{2.9-x} B r_{x} C l_{0.1}$ perovskite, fixing the chloride content and varying the iodide and bromide content, resulting in enhanced film morphology with a strong absorbance in the $U V$-vis range. Also, the Pawley fit method indicated the formation of the iodide halide $\mathrm{MAPbI}_{3} P m \overline{3} m$ cubic phase for $x=0$ and tetragonal $P 4 / \mathrm{mmm}$ phase for $x \geq 0.3$. It was found that both $C l$ and $\mathrm{Br}$ affect the $\mathrm{PL}$ emission of the $M A P b I_{2.9-x} B r_{x} C l_{0.1}$ mixed halide lead perovskite. 
Fourth: since the presence of the organic cation inevitably decreases its stability, it is necessary to test inorganic cations, such as cesium aiming at increased stability. In this light, inorganic cesium lead halide $\mathrm{CsPbX}_{3}$ perovskite was studied in depth. Following this line of research, we synthesized mixed cesium lead halide $\mathrm{CsPbBr}_{3-x} I_{x}$ perovskite thin films, with the objective to study the role of iodide partial substitution on the physical properties and stability. We reported that $C s P b B r_{3-x} I_{x}$ films exhibit a very high absorbance in the visible spectrum and short infrared region and, in addition, the color of perovskite films can be tuned from yellow to dark brown when increasing the iodide content in mixed halide perovskites. As expected, $\mathrm{CsPbBr}_{3-x} I_{x}$ showed an improved resistance to humidity and heat, indicating that mixed cesium lead halide perovskites represent a promising photovoltaic material.

Fifth: aiming at the improvement of the stability, efficiency and morphology of methylammonium based perovskites, we focused on the partial substitution of $M A$ with other organic cations, such as the guanidinium (GA) and imidazolium (IM) cations. Mixed $M A_{1-x} G A_{x} P b I_{3}$ and $M A_{1-x} I M_{x} P b I_{3}$ perovskites showed very promising optical and morphological properties. $X R D$ results on $M A_{0.9} G A_{0.1} P b I_{3}$ showed a significantly improved stability and resistance to moisture and humidity after eight months. Samples with a $G A$ content between 0 and $20 \%$ showed a very slight shift towards lower energies, with an estimated bandgap from 1.60 to $1.59 \mathrm{eV}$. Also, it was found that as the amount of $G A$ content increases, the thin films become more homogeneous, at the cost of losing absorption and efficiency. The same pattern of improvement can be observed when an anti-solvent is employed, obtaining more uniform and smoother grain-shape surfaces, and corroborating the importance of employing anti-solvents on the perovskite crystal growth 
kinetics and film morphology.

The imidazolium cation showed similar results. $X$-ray diffraction analyses established the limit of solubility of $I M$ at about $10 \%$. Mixed perovskites with an $I M$ content below $10 \%$, and specifically the $M A_{0.94} I M_{0.06} P_{b I}$ thin film, showed an improvement in crystallinity. $P L$ results suggested that $I M$ incorporation reduces nonradiative recombination significantly and acts as an effective passivator. FESEM analysis showed an improved morphology for the mixed $M A_{1-x} I M_{x} P b I_{3}$ thin films.

Sixth: finally, the fabrication of $\mathrm{MAPbI}_{3}$ perovskite solar cells, showing a high efficiency of about $15 \%$, by a one-step spin-coating process in ambient air is reported. A comparison of the performance parameters, before and after spiro-OMeTAD oxidation for $24 h$ is presented, indicating that oxidation of spiro-OMeTAD, under controlled humidity conditions, can enhance performance parameters. Each pixel on the devices showed similar efficiencies, demonstrating a high reproducibility. The crystallization with diethyl ether was carried out successfully, obtaining a homogeneous layer of bright amber color. These results make the one-step spin-coating process in air a cost-effective competitive candidate for high efficiency solar cells fabrication, when compared to other vacuum manufacturing methods. 


\section{Bibliography}

[1] IPCC Fifth Assessment Report, Summary for Policymakers

[2] B.D. Santer et.al. Nature vol 382, (1996), 39-46.

[3] Gabriele C. Hegerl. Journal of Climate, v. 9, October (1996), 2281-2306.

[4] V. Ramaswamy et.al. Science 311 (2006), 1138-1141.

[5] B.D. Santer et.al. Science vol. 301 (2003), 479-483.

[6] National Research Council (NRC). National Academy Press, Washington, D.C., (2006).

[7] National Aeronautics and Space Administration, Goddard Institute for Space Studies. http://data.giss.nasa.gov/gistemp [Last Access: June 26, 2018].

[8] National Oceanic and Atmospheric Administration. https://www.ncdc.noaa.gov/indicators/ [Last Access: June 26, 2018].

[9] GISS NASA. https://data.giss.nasa.gov/gistemp/graphs/ [Last Access: June $26,2018]$.

[10] United Nations Framework Convention on Climate Change, Paris Agreement (2015).

[11] US Energy Information Administration, AEO2013 Early Release Overview (2013).

[12] United Nations, General Assembly, 2015. 
[13] W. Collins, R. Colman, J. Haywood, M. R. Manning and P. Mote, Scientific American 297 (2007), 64-73.

[14] A. Pockett. Characterization of Perovskite Solar Cells. Degree of Doctor of Philosophy, University of Bath, Department of Chemistry (2016).

[15] N.S. Lewis, MRS Bull. 32, (2007).

[16] European Photovoltaic Industry Association Solar Generation 6, (2011), 14.

[17] Earth Observatory NASA. http://earthobservatory.nasa.gov/Features [Last Access: June 26, 2018]

[18] APS News. American Physical Society. 18 (4) (2009).

[19] National Center for Photovoltaics (U.S. Department of Energy), Research Cell Efficiency Records Chart. https://www.nrel.gov/pv/assets/images/efficiency-chart.png [Last Access: June 26, 2018].

[20] Seeking Alpha. http://seekingalpha.com/article [Last Access: June 28, 2018].

[21] Safdari. Ph.D. Thesis. TRITA-CHE-Report, (2017).

[22] U. Eva. Excitonic Dye Solar Cells. PhD thesis, Uppsala University, (2012).

[23] A. Lentz, D. Renné, N. Romeo, A. Bosio, D. Menossi, A. Romeo, M. Aramini, 2013 ISES Solar World CongressLast Progress in CdTe/CdS Thin Film Solar Cell Fabrication Process. Energy Procedia, 57, (2014), 65-72.

[24] S.G. Kumar, K. Rao. Energy \& Environmental Science, 7(1) (2014), 45-102.

[25] L.L. Kazmerski. Journal of Electron Spectroscopy and Related Phenomena 150(2-3) (2006), 105-135.

[26] P. Jackson, D. Hariskos, R. Wuerz, O. Kiowski, A. Bauer, T.M. Friedlmeier, M. Powalla. Physica Status Solidi (RRL)- Rapid Research Letters, 9(1) (2015), 28-31. 
[27] U.P. Singh, S.P. Patra. International Journal of Photoenergy 2010, (2010).

[28] N. Vlachopoulos, P. Liska, J. Augustynski and M. Graetzel, J. Am. Chem. Soc., 110 (1988), 1216-1220.

[29] B. O’Regan and M. Grätzel, Nature, 353 (1991), 737-740.

[30] J.-H. Yum, E. Baranoff, F. Kessler, T. Moehl, S. Ahmad, T. Bessho, A. Marchioro, E. Ghadiri, J.-E. Moser, C. Yi, M. K. Nazeeruddin and M. Grätzel. Nat Commun, 3 (2012), 631.

[31] K. Kakiage, Y. Aoyama, T. Yano, K. Oya, J.-I. Fujisawa and M. Hanaya. Chem. Commun., 51 (2015), 15894-15897.

[32] J. Burschka, A. Dualeh, F. Kessler, E. Baranoff, N.-L. Cevey-Ha, C. Yi, M. K. Nazeeruddin and M. Grätzel. J. Am. Chem. Soc., 133 (2011), 18042-18045.

[33] H. J. Snaith and L. Schmidt-Mende, Adv. Mater.,19 (2007), 3187-3200.

[34] D.B. Mitzi, K. Chondroudis, C.R. Kagan. IBM Journal of Research and Development 45(1) (2001) 29-45.

[35] D.B. Mitzi. Inorganic Chemistry 39(26) (2000) 6107-6113.

[36] B.-W. Park, B. Philippe, X. Zhang, H. Rensmo, G. Boschloo, E.M.J. Johansson. Advanced Materials 27(43) (2015) 6806-6813.

[37] C.C. Stoumpos, L. Frazer, D.J. Clark, Y.S. Kim, S.H. Rhim, A.J. Freeman, J.B. Ketterson, J.I. Jang, M.G. Kanatzidis. Journal of the American Chemical Society 137(21) (2015) 6804-6819.

[38] A. Poglitsch, D. Weber. Journal of Chemical Physics 87(11) (1987) 6373-6378.

[39] T. Baikie, Y. Fang, J.M. Kadro, M. Schreyer, F. Wei, S.G. Mhaisalkar, M. Graetzel, T.J. White. J. Mater. Chem. A 1 (2013) 5628-5641.

[40] C.C. Stoumpos, C.D. Malliakas, M.G. Kanatzidis. Inorganic Chemistry 52(15) (2013) 9019-9038. 
[41] D.B. Mitzi, K. Chondroudis, C.R. Kagan. Inorganic Chemistry 38(26) (1999) 6246-6256.

[42] D.B. Mitzi. Progress in Inorganic Chemistry. John Wiley \& Sons, Inc. (2007) $1-121$.

[43] R.J.D. Tilley, Perovskites: Structure-Property Relationships. ProQuest Ebook Central. John Wiley \& Sons, Incorporated (2016).

[44] N.-G. Park, M. Gratzel \& T. Miyasaka. Organic-Inorganic Halide Perovskite Photovoltaics (1st ed. 2016 ed.). Springer Verlag.(2016).

[45] S. Gholipour, A.M. Ali, J.P. Correa-Baena. Adv. Mater. (2017). 1702005. DOI: 10.1002/adma.201702005.

[46] A. Kojima, K. Teshima, Y. Shirai and T. Miyasaka, J. Am. Chem. Soc. 131 (2009) 6050-6051.

[47] J.H. Im, C.R. Lee, J.W. Park, N.G. Park. Nanoscale 3 (2011) 4088-4093. http://dx.doi.org/10.1039/C1NR10867K.

[48] I. Chung, B. Lee, J. He, R. P. H. Chang and M. G. Kanatzidis, Nature 485 (2012) 486-489.

[49] H.-S. Kim, C.-R. Lee, J.-H. Im, K.-B. Lee, T. Moehl, A. Marchioro, S.-J. Moon, R.H.-Baker, J.-H. Yum, J.E. Moser, M. Gratzel, N.-G. Park. Sci. Rep. 2 (2012) $1-7$.

[50] L. Etgar, P. Gao, Z. Xue, Q. Peng, A. K. Chandiran, B. Liu, M. K. Nazeeruddin and M. Grätzel. J. Am. Chem. Soc. 134 (2012) 17396-17399. https://doi.org/10.1021/ja307789s.

[51] M.M. Lee, J. Teuscher, T. Miyasaka, T.N. Murakami and H. J. Snaith. Science 338 (2012) 643-647.

[52] J. Burschka, N. Pellet, S.-J. Moon, R. Humphry-Baker, P. Gao, M. K. Nazeeruddin and M. Gratzel. Nature 499 (2013) 316-319. https://doi.org/10.1038/nature12340. 
[53] M. Liu, M.B. Johnston, H.J. Snaith. Nature 501 (2013) 395-398.

[54] S. Colella, E. Mosconi, P. Fedeli, A. Listorti, F. Gazza, F. Orlandi, P. Ferro, T. Besagni, A. Rizzo, G. Calestani, G. Gigli, F. De Angelis and R. Mosca. Chem. Mater. 25 (2013) 4613-4618.

[55] J.H. Noh, S.H. Im, J.H. Heo, T.N. Mandal, S.I. Seok. Nano Lett. 13 (2013) 1764-1769.

[56] Q. Chen, H. Zhou, Z. Hong, S. LuO, H.-Sh Duan, H.-H. Wang, Y. Liu, G.- Li, Y. Yang. J. Am. Chem. Soc. 136(2) (2014) 622-625. http://dx.doi.org/10.1021/ja411509g.

[57] J. Burschka, N. Pellet, S.-J. Moon, K.H. Baker, P. GaO, M.K. Nazeeruddin, M. Grätzel. Nature 499 (2013) 316-319. http://dx.doi.org/10.1038/nature12340.

[58] Y. Zhao, K. Zhu. J. Mater. Chem. A 3(17) (2015) 9086-9091. http://dx.doi.org/10.1039/C4TA05384B.

[59] J.T.W. Wang, J.M. Ball, E.M. Barea, A. Abate, J.A. Alexander-Webber, J. Huang, M. Saliba, I. Mora-Sero, J. Biqueret, H.J. Snaith, K.J. Nicholas. Nano Lett. 14(2) (2014) 724-730. http://dx.doi.org/10.1021/nl403997a.

[60] K. Wojciechowski, M. Saliba, T. Leijtens, A. Abate, H.J. Snaith. Energy Environ. Sci. 7(3) (2014) 1142-1147. http://dx.doi.org/10.1039/C3EE43707H.

[61] N.J. Jeon, J.H. Noh, Y.C. Kim, W.S. Yang, S. Ryu, S.I. Seok. Nat. Mater. 13 (2014) 897-903. http://dx.doi.org/10.1038/nmat4014.

[62] N.J. Jeon, H.G. Lee, Y.C. Kim, J. Seo, J.H. Noh, J. Lee, S.I. Seok. Chem. Soc. 136(22) (2014) 7837-7840. http://dx.doi.org/10.1021/ja502824c.

[63] H. Zhou, Q. Chen, G. Li, S. Luo, T.B. Song, H.S. Duan, Z. Hong, J. You, Y. Liu, Y. Yang. Science 345 (6196) (2014) 542-546. http://dx.doi.org/10.1126/science.1254050.

[64] D. Weber. Zeitschrift für Naturforsch. 33b (1978) 862-865. 
[65] R. Wasylishen, O. Knop, J. Macdonald. Solid State Commun. 56(7) (1985) 581-582.

[66] N. Onoda-Yamamuro, O. Yamamuro, T. Matsuo, H. Suga. J. Phys. Chem. Solids 53(2) (1992) 277-281.

[67] A. Poglitsch, D. Weber. J. Chem. Phys. 87 (1987) 6373.

[68] M.T. Weller, O.J. Weber, P.F. Henry, A.M. Di Pumpo, T.C. Hansen. Chem. Commun. 51 (2015) 4180-4183.

[69] R.J. Worhatch, H.J. Kim, I.P. Swainson, A.L. Yonkeu, S.J.L. Billinge. Chem. Mater. 20 (2008) 1272-1277.

[70] M.I. Asghara, J. Zhangb, H. Wangb, P.D. Lunda,bRenewable and Sustainable Energy Reviews 77 (2017) 131-146.

[71] Jeanbourquin XA, Li X, Law C, Barnes PRF, Humphry-Baker R, Lund P, O'Regan BC. J Am Chem Soc (2014);136:7286-94. http://dx.doi.org/10.1021/ja411560s.

[72] Chen W, Wu Y, Yue Y, Liu J, Zhang W, Yang X, Chen H, Bi E, Ashraful I, Grätzel M, Han L. Efficient and stable large-area perovskite solar cells with inorganic charge extraction layers. Science 2015;350:944-8.

[73] N. Rolston, A.D. Printz, J.M. Tracy, H. C. Weerasinghe, D. Vak, L.J. Haur, A. Priyadarshi, N. Mathews, D.J. Slotcavage, M.D. McGehee, R.E. Kalan, K. Zielinski, R.L. Grimm, H. Tsai, W. Nie, A.D. Mohite, S. Gholipour, M. Saliba, M. Grätzel, and R.H. Dauskardt. Adv. Energy Mater. 2018, 8, 1702116.

[74] G. Niu, X. Guo, L. Wang, J. Mater. Chem. A 3 (2015) 8970-8980.

[75] J.M. Ball, M.M. Lee, A. Hey, H.J. Snaith, Low-temperature processed meso-superstructured to thin-film perovskite solar cells, Energy Environ. Sci. 6 (2013) 1739-1743.

[76] P. Schouwink, M. B. Ley, A. Tissot, H. Hagemann, T. R. Jensen, L. Smrcok and R. Cerny, Nature Communications, 2014, 5, 1-10. 
[77] Bach U, Lupo D, Comte P, Moser JE, Weissörtel F, Salbeck J, Spreitzer H, Grätzel M. Solid-state dye-sensitized mesoporous TiO2 solar cells with high photon-toelectron conversion efficiencies. Nature 1998;395:583-5.

[78] Eperon GE, Stranks SD, Menelaou C, Johnston MB, Herz LM, Snaith HJ. Formamidinium lead trihalide: a broadly tunable perovskite for efficient planar heterojunction solar cells. Energy Environ Sci 2014;7:982-8.

[79] A. Binek, F. C. Hanusch, P. Docampo and T. Bein, J. Phys. Chem. Lett., 2015, $6,1249-1253$.

[80] N. J. Jeon, J. H. Noh, W. S. Yang, Y. C. Kim, S. Ryu, J. Seo and S. I. Seok, Nature, 2015, 517, 476-480.

[81] Z. Li, M. Yang, J.-S. Park, S.-H. Wei, J. J. Berry and K. Zhu, Chem. Mater., 2016, 28, 284-292.

[82] T. Jesper Jacobsson, J.-P. Correa-Baena, M. Pazoki, M. Saliba, K. Schenk, M. Gratzel and A. Hagfeldt, Energy Environ. Sci., 2016, 9, 1706-1724.

[83] M. Saliba, T. Matsui, J.-Y. Seo, K. Domanski, J.-P. Correa-Baena, M. K. Nazeeruddin, S. M. Zakeeruddin, W. Tress, A. Abate, A. Hagfeldt and M. Gratzel, Energy Environ. Sci., 2016, 9, 1989-1997.

[84] C. K. Møller, Nature, 1958, 182, 1436-1436.

[85] Z. Li, M. Yang, J. S. Park, S. H. Wei, J. J. Berry and K. Zhu, Chemistry of Materials, 2016, 28, 284-292.

[86] M. Kaltenbrunner, G. Adam, E. D. Glowacki, M. Drack, R. Schwodiauer, L. Leonat, D. H. Apaydin, H. Groiss, M. C. Scharber, M. S. White, N. S. Sarici and S. Bauer, Nat. Mater., 2015, 14, 1032-1039.

[87] T. Leijtens, G. E. Eperon, A. J. Barker, G. Grancini, W. Zhang, J. M. Ball, A. R. S. Kandada, H. J. Snaith and A. Petrozza, Energy Environ. Sci., 2016, $9,3472-3481$. 
[88] C. Liu et al., A brief review on the lead element substitution in perovskite solar cells, Journal of Energy Chemistry (2017), https://doi.org/10.1016/j.jechem.2017.10.028.

[89] J.-P. Correa-Baena , A. Abate, M. Saliba , W. Tress , T. Jesper Jacobsson , M. Grätzel , A. Hagfeldt, Energy Environ. Sci. 10 (2017) 710-727.

[90] E. Union, Environment : EU ban on hazardous substances in electrical and electronic products takes effect, 2006.

[91] A . Babayigit, A . Ethirajan , M. Muller , B. Conings. Nat. Mater. 15 (2016) $247-251$.

[92] T. Umebayashi, K. Asai, T. Kondo, A. Nakao. Physical Review B 67 (2003) 155405.

[93] P.P. Boix , S. Agarwala, T.M. Koh , N. Mathews, S.G. Mhaisalkar ,J. Phys. Chem. Lett. 6 (2015) 898-907.

[94] F. Giustino , H.J. Snaith , ACS Energy Lett. 1 (2016) 1233-1240.

[95] D. Weber, Zeitschrift für Naturforschung, 1978, 33b, 862-865.

[96] F. Zuo , S.T. Williams , P.W. Liang, C.C. Chueh , C.Y. Liao , A.K.Y. Jen ,Adv. Mater. 26 (2014) 6454-6460.

[97] N. K. Noel, S. D. Stranks, A. Abate, C. Wehrenfennig, S. Guarnera, A.-A. Haghighirad, A. Sadhanala, G. E. Eperon, S. K. Pathak, M. B. Johnston, A. Petrozza, L. M. Herz and H. J. Snaith, Energy Environ. Sci., 2014, 7, 3061-3068.

[98] F. Hao, C. C. Stoumpos, D. H. Cao, R. P. H. Chang and M. G. Kanatzidis, Nature Photonics, 2014, 8, 489-494.

[99] K. Shum, Z. Chen, J. Qureshi, C. Yu, J.J. Wang, W. Pfenninger, N. Vockic , J. Midgley , J.T. Kenney , Appl. Phys. Lett. 96 (2010) 221903. 
[100] I. Chung , J.H. Song , J. Im , J. Androulakis , C.D. Malliakas , H. Li , A.J. Freeman , J.T. Kenney , M.G. Kanatzidis , J. Am. Chem. Soc. 134 (2012) 8579-8587.

[101] F. Chiarella, A. Zappettini, F. Licci, I. Borriello, G. Cantele, D. Ninno, A. Cassi- nese, R. Vaglio, Phys. Rev. B 77 (2008) 045129.

[102] K. Yamada, S. Nose , T. Umehara, T. Okuda, S. Ichiba, Bull. Chem. Soc. Jpn. 61 (1988) 4265-4268.

[103] D.E. Scaife , P.F. Weller , W.G. Fisher, J. Solid State Chem. 9 (1974) 308.

[104] L.J. Chen, C.R. Lee, Y.J. Chuang, Z.H. Wu, C. Chen. Journal of Physical Chemistry Letters, 7 (2016) 5028-5035.

[105] J. Liang, P. Zhao , C. Wang, Y. Wang, Y. Hu , G. Zhu , L. Ma , J. Liu , Z. Jin. J. Am. Chem. Soc., 139 (2017) 14009-14012.

[106] Y. Li, W. Sun, W. Yan, S. Ye, H. Rao, H. Peng, Z. Zhao, Z. Bian, Z. Liu, H. Zhou, C. Huang. Advanced Energy Materials, 6(24) (2016) 1601353.

[107] P.-Y. Chen, J. Qi, M.T. Klug, X. Dang, P.T. Hammond, A.M. Belcher. Energy Environ. Sci., 7 (2014) 3659-3665.

[108] M.A. Green, A. Ho-Baillie, H.J. Snaith. Nat. Photonics 8 (2014) 506514. http://dx.doi.org/10.1038/nphoton.2014.134.

[109] G. Kieslich, S. Sun, A. K. Cheetham. Chem. Sci. 5 (2014) 4712-4715. https://doi.org/10.1039/c4sc02211d.

[110] D. M. Nicholas, Z. Huanping, C. Qi, S. Pengyu, L. Zonghao, M. Lei, Y. En-Ping, S. Andy, Y. Yang. Nano Lett. 16 (2016) 1009.

[111] R. Shannon. Acta Crystallogr. Sect A 32(5) (1976) 751-767.

[112] C. Bartel1, C. Sutton, B. R. Goldsmith, R. Ouyang, C. B. Musgrave, L. M. Ghiringhelli, M. Scheffler. arXiv:1801.07700 [cond-mat.mtrl-sci] 
[113] Perovskite stability. https://github.com/CJBartel/perovskite-stability. Last access june 142018 .

[114] E. H. Anaraki, A. Kermanpur, L. Steier, K. Domanski, T. Matsui, W. Tress, M. Saliba, A. Abate, M. Grätzel, A. Hagfeldt, J.-P. Correa-Baena. Energy Environ. Sci., vol. 9, no. 10 (2016) 3128-3134.

[115] J. P. Correa Baena, L. Steier, W. Tress, M. Saliba, S. Neutzner, T. Matsui, F. Giordano, T. J. Jacobsson, A. R. Srimath Kandada, S.M. Zakeeruddin, A. Petrozza, A. Abate, M. K. Nazeeruddin, M. Grätzel, and A. Hagfeldt. Energy Environ. Sci., vol. 8, no. 10, (2015) 2928-2934.

[116] A. Yella, L.-P.Heiniger, P. Gao, M. K.Nazeeruddin, and M. Grätzel. Nano Letters 14 (2014) 2591-2596.

[117] J. Burschka, N. Pellet, S.-J.Moon, R. Humphry-Baker, P. Gao, M. K. Nazeeruddin, M. Grätzel. Nature 499 (2013) 316-319.

[118] G. Xing, N. Mathews, S. Sun, S.S. Lim, Y.M. Lam, M. Grätzel, S. Mhaisalkar, T.C. Sum. Science 342 (2013) 344-347.

[119] D.-Y. Son, J.-H. Im, H.-S. Kim, and N.-G. Park. The Journal of Physical Chemistry C, 118 (2014) 16567-16573.

[120] D. Liu, T.L. Kelly. Nat. Photon., 8 (2013) 133-138.

[121] D. Bi, S.-J.Moon, L. Häggman, G. Boschloo, L. Yang, E. M. J. Johansson, M. K. Nazeeruddin, M. Grätzel, A. Hagfeldt. RSC Advances, 3(41) (2013) 18762.

[122] M. A. Green, A. Ho-Baillie, H. J. Snaith. Nature Photonics, 8 (2014) 506-514.

[123] S. D. Stranks, H. J. Snaith. Nature Nanotechnology, 10 (2015) 391-402.

[124] U. Bach, D. Lupo, P. Comte, J. E. Moser, F. Weissörtel, J. Salbeck, H. Spreitzer, M. Grätzel. Nature 395 (1998) 583-585.

[125] J. You , Y. M. Yang, Z. Hong, T.-B. Song , L. Meng, Y. Liu , C. Jiang , H. Zhou , W.-H. Chang, G. Li , Y. Yang. Appl. Phys. Lett. 105 (2014) 183902. 
[126] H. Gao , C. Bao, F. Li, T. Yu, J. Yang, W. Zhu, X. Zhou , G. Fu , Z. Zou. ACS Appl. Mater. Interfaces 7 (2015) 9110.

[127] A. M. A. Leguy , Y. Hu , M. Campoy-Quiles , M. I. Alonso , O. J. Weber , P. Azarhoosh, M. V. Schilfgaarde, M. T. Weller, T. Bein , J. Nelson, P. Docampo , P. R. F. Barnes. Chem. Mater. 27 (2015) 3397.

[128] J. F. Galisteo-López , M. Anaya , M. E. Calvo , H. Míguez. J. Phys. Chem. Lett. 6 (2015) 2200 .

[129] S. R. Raga , M.-C. Jung, M. V. Lee, M. R. Leyden, Y. Kato, Y. Qi. Chem. Mater. 27 (2015) 1597.

[130] C. D. Bailie, M. G. Christoforo, J. P. Mailoa, A. R. Bowring, E. L. Unger, W. H. Nguyen, J. Burschka, N. Pellet, J. Z. Lee, M. Grätzel, R. Noufi, T. Buonassisi, A. Salleo, M. D. McGehee. Energy Environ. Sci., 8 (3) (2015) 956-963.

[131] J. Jang, H.-G. Im, J. Jin, J. Lee, J.-Y. Lee, and B.-S. Bae. ACS AppliedMaterials \& Interfaces 8 (2016) 27035-27043.

[132] J.Werner, C.-H.Weng, A.Walter, L. Fesquet, J. P. Seif, S. DeWolf, B. Niesen, and C. Ballif. The Journal of Physical Chemistry Letters, 7 (2016) 161-166.

[133] P. Löper, S.-J.Moon, S.Martín de Nicolas, B.Niesen, M. Ledinsky, S.Nicolay, J. Bailat, J.-H. Yum, S. DeWolf, and C. Ballif. Chem. Phys., 17(3) (2015) 1619-1629.

[134] K. L. Gardner, J. G. Tait, T. Merckx, W. Qiu, U. W. Paetzold, L. Kootstra, M. Jaysankar, R. Gehlhaar, D. Cheyns, P. Heremans, J. Poortmans, Advanced Energy Materials 6 (2016) 1-8.

[135] A. Dualeh, N. Tétreault, T. Moehl, P. Gao, M. K. Nazeeruddin, M. Grätzel. Advanced Functional Materials, 24 (2014) 3250-3258.

[136] K. Liang, D. B. Mitzi, M. T. Prikas. Chemistry of Materials, 10 (1998) 403-411.

[137] J. Burschka, N. Pellet, S.-J. Moon, R. Humphry-Baker, P. Gao, M. K. Nazeeruddin, M. Grätzel. Nature, 499 (2013) 316-319. 
[138] H. Chen. Advanced Functional Materials, 27 (2017).

[139] J.-H. Im, I.-H. Jang, N. Pellet, M. Grätzel, N.-G. Park. Nature nanotechnology, (2014).

[140] M. R. Leyden, L. K. Ono, S. R. Raga, Y. Kato, S. Wang, Y. Qi. J. Mater. Chem. A, 2(44) (2014) 18742-18745.

[141] Q. Chen,H. Zhou, Z.Hong, S. Luo, H.-S.Duan, H.-H.Wang, Y. Liu, G. Li, Y. Yang. Journal of the American Chemical Society, 136 (2014) 622-625.

[142] H. Zhou, Q. Chen, Y. Yang. MRS Bulletin, 40 (2105) 667-673.

[143] K. Chondroudis, D. Mitzi. Chemistry of materials, 11 (1999) 3028-3030.

[144] D.B.Mitzi, P. Brock. Inorganic chemistry, 40 (2001) 2096-104.

[145] K. Chondroudis, D. B.Mitzi, P. Brock. Chemistry of Materials, 12 (2000) 169-175.

[146] O.Malinkiewicz, A. Yella, Y. H. Lee, G. M. Espallargas, M. Graetzel, M. K. Nazeeruddin, H. J. Bolink. Nature Photonics, 8 (2013) 128-132.

[147] M. Liu, M. B. Johnston, and H. J. Snaith. Nature, 501 (2013) 395-398.

[148] C. Momblona, O. Malinkiewicz, C. Roldán-Carmona, A. Soriano, L. Gil-Escrig, E. Bandiello, M. Scheepers, E. Edri, H. J. Bolink. APL Materials, 2 (2014) 081504.

[149] J. Teuscher, A. Ulianov, O. Müntener, M. Grätzel, N. Tétreault. Chem Sus Chem, 8 (2015) 3847-3852.

[150] C. Gao, J. Liu, C. Liao, Q. Ye, Y. Zhang, X. He, X. Guo, J.Mei, W. Lau. RSC Adv., 5(33) (2015) 26175-26180.

[151] G.-X. Liang, P. Fan, J.-T. Luo, D. Gu, Z.-H. Zheng. Progress in Photovoltaics: Research and Applications, 23 (2015) 1901-1907. 
[152] G. Longo, L. Gil-Escrig, M. J. Degen,M. Sessolo, H. J. Bolink. Chem. Commun., 51(34) (2015) 7376-7378.

[153] X. Li, D. Bi, C. Yi, J.-D. Decoppet, J. Luo, S. M. Zakeeruddin, A. Hagfeldt, M. Gratzel. Science, (2016).

[154] M. Saliba, K. W. Tan , H. Sai, D. T. Moore, T. Scott, W. Zhang, L. A. Estroff , U. Wiesner , H. J. Snaith , J. Phys. Chem. C 118 (2014) 17171.

[155] C. Bi , Y. Shao, Y. Yuan, Z. Xiao, C. Wang, Y. Gao, J. Huang, J. Mater. Chem. A 2 (2014) 18508.

[156] R. Kang , J.-E. Kim , J.-S. Yeo, S. Lee, Y.-J. Jeon , D.-Y. Kim , J. Phys. Chem. C 118 (2014) 26513.

[157] Z. Xiao , Q. Dong, C. Bi , Y. Shao, Y. Yuan , J. Huang , Adv. Mater. 26 (2014) 6503 .

[158] H. Yu , X. Liu , Y. Xia, Q. Dong, K. Zhang, Z. Wang, Y. Zhou , B. Song , Y. Li. J. Mater. Chem. A 4 (2016) 321.

[159] J. Liu , C. Cao , X. He, Q. Ye , L. Ouyang, D. Zhuang , C. Liao , J. Wei , W. Lau. ACS Appl. Mater. Interfaces 7 (2015) 24008.

[160] D. Liu , L. Wu , C. Li , S. Ren, J. Zhang, W. Li , L. Feng. ACS Appl. Mater. Interfaces 7 (2015) 16330.

[161] F. Huang, Y. Dkhissi, W. Huang, M. Xiao, I. Benesperi, S. Rubanov, Y. Zhu, X. Lin, L. Jiang, Y. Zhou. Nano Energy 10 (2014) 10-18.

[162] Y. Zhao, K.J. Zhu. Phys. Chem. C 118 (2014) 9412-9418.

[163] C. Zuo, L. Ding. Nanoscale 6 (2014) 9935-9938.

[164] D. Bi, P. Gao, R. Scopelliti, E. Oveisi, J. Luo, M. Grätzel, A. Hagfeldt, M.K. Nazeeruddin. Adv. Mater. 28 (2016) 2910-2915.

[165] M. Xiao, F. Huang, W Huang, Y. Dkhissi, Y.Zhu, J. Etheridge, A. Gray-Weale, U. Bach, Y.B. Cheng, L. Spiccia. Angew. Chem., Int. Ed. 53 (2014) 9898-9903. 
[166] N.J. Jeon, J.H. Noh, Y.C. Kim, W.S. Yang, S. Ryu, S. Seok. Nat. Mater. 13 (2014) 897-903.

[167] Z. Xiao, Q. Dong, C. Bi, Y. Shao, Y. Yuan, J. Huang. Adv. Mater. 26 (2014) 6503-6509.

[168] Y.H. Lee, J. Luo, R. Humphry-Baker, P. Gao, M. Grätzel, M.K. Nazeeruddin. Adv. Funct. Mater. 25 (2015) 3925-3933.

[169] F.X. Xie, D. Zhang, H. Su, X. Ren, K. Wong, M. Grätzel, W.C.H Choy. ACS Nano 9 (2015) 639-646.

[170] N. J. Jeon, J. H. Noh, Y. C. Kim, W. S. Yang, S. Ryu and S. Il Seok. Nature Materials 13 (2014) 897-903.

[171] M. Saliba, T. Matsui, K. Domanski, J.-Y. Seo, A. Ummadisingu, S. M. Zakeeruddin, J.-P. Correa-Baena, W. R. Tress, A. Abate, A. Hagfeldt, M. Grätzel. Science 354 (2016).

[172] Y. Gao, L. Yang, F. Wang, Y. Sui, Y. Sun, M. Wei, J. Cao, H. Liu. Superlattices and Microstructures, 2017.

[173] N.-G. Park, Nano Convergence 3 (2016) 15.

[174] N.J. Jeon, J.H. Noh, W.S. Yang, Y.C. Kim, S. Ryu, J. Seo, S. Il Seok. Nature 517 (2015) 476-480.

[175] W.S. Yang, J.H. Noh, N.J. Jeon, Y.C. Kim, S. Ryu, J. Seo, S. Il Seok. Science (Washington, DC, U. S.) 348 (2015) 1234-1237.

[176] D. Bi, W. Tress, M.I. Dar, P. Gao, J. Luo, C. Renevier, K. Schenk, A. Abate, F. Giordano, J.P. Correa Baena. Sci. Adv. 2 (2016) 1501170-1501170.

[177] Y. C. Kim, N. J. Jeon, J.H. Noh, W.S. Yang, J. Seo, J.S. Yun, A. Ho-Baillie, S. Huang, M.A. Green, J. Seidel et al. Adv. Energy Mater. 6 (2016) 1502104.

[178] D.-Y. Son, J.-W. Lee, Y. J. Choi, I.-H. Jang, S. Lee, P.J. Yoo, H. Shin, N. Ahn, M. Choi, D. Kim, et al. Nat. Energy 1 (2016) 16081-16088. 
[179] S. Paek, P. Schouwink, E. Nefeli Athanasopoulou, K. T. Cho, G. Grancini, Y. Lee, Y. Zhang, F. Stellacci, Mohammad Khaja Nazeeruddin,P. Gao. Chem. Mater. 29 (2017) 3490-3498.

[180] A. Ng , Z. Ren , Q. Shen , S. H. Cheung, H. C. Gokkaya , G. Bai , J. Wang , L. Yang, S. K. So , A. B. Djurisic, W. W.-f. Leung , J. Hao , W. K. Chanf, C. Surya, J. Mater. Chem. A 3 (2015) 9223.

[181] Z. Ren, A. Ng, Q. Shen, H.C. Gokkaya, J. Wang, L. Yang, W.-K. Yiu, G. Bai, A.B. Djurisic, W.W.f. Leung, J. Hao, W.K. Chan, C. Surya. Sci. Rep. 4 (2014) 6752 .

[182] B. Yang, O. Dyck, J. Poplawsky , J. Keum, S. Das , A. Puretzky , T. Aytug , P. C. Joshi, C. M. Rouleau, G. Guscher, D. B. Geohegan, K. Xiao. Angew. Chem. Int. Ed. 54 (2015) 14862.

[183] S. Pathak , A. Sepe , A. Sadhanala , F. Deschler , A. Haghighirad , N. Sakai , K. C. Goedel, S. D. Stranks , N. Noel, M. Price, S. Hüttner , N. A. Hawkins , R. H. Friend, U. Steiner, H. J. Snaith. ACS Nano 9 (2015) 2311.

[184] M. Green. Solar Cells: Operating Principles, Technology, and System Applications, Prentice Hall, Englewood Cliffs, NJ, (1982) 1-12.

[185] Handbook of Photovoltaic Science and Engineering, Second Edition. Edited by Antonio Luque and Steven Hegedus. John Wiley \& Sons, Ltd. (2011).

[186] M. Fox. Optical Properties of Solids. Oxford University Press. New York (2010).

[187] P. Harlan. Nanostructured Photovoltaics: Improving Device Efficiency and Measuring Carrier Transport. Ph.D. Massachusetts Institute of Technology (2017).

[188] W. Shockley and H. J. Queisser. Journal of Applied Physics, 32 (1961) 510-519.

[189] M. A. Green. Progress in Photovoltaics: Research and Applications, 20 (2012) 954-959. 
[190] K. Sveinbjörnsson. Preparation and Characterization of Lead Halide Perovskites. Ph.D. Acta Universitatis Upsaliensis. Uppsala (2018).

[191] P. Atkinson, J. de Paula, Physical Chemistry, Volume 1 (10th Edition). Oxford University Press (2014).

[192] R.F. Pierret. Semiconductor Device Fundamentals. Addison-Wesley, Reading, MA, (1996).

[193] K.W. Johnston, A.G. Pattantyus-Abraham, J.P. Clifford, S.H. Myrskog, S. Hoogland, H. Shukla, E.J.D. Klem, L. Levina, and E.H. Sargent. Appl. Phys. Lett. 92 (2008) 122111.

[194] A.L. Fahrenbruch and R.H. Bube. Fundamentals of Solar Cells: Photovoltaic Solar Energy Conversion. Academic Press, New York (1983).

[195] P. Würfel and U. Würfel. Physics of Solar Cells: From Basic Principles to Advanced Concepts (2nd ed.). Wiley-VCH, Weinheim; Norwood, MA (2009).

[196] S. Rühle. Sol. Energy 130 (2016) 139-147.

[197] A. De Vos. J. Phys. D. Appl. Phys. 13 (1980) 839-846.

[198] T. Umebayashi, K. Asai, T. Kondo and A. Nakao. Physical Review B, 67 (2003) 155405 .

[199] K. T. Butler, J. M. Frost and A. Walsh. Material Horizons, 2 (2015) 228-231.

[200] F. Brivio, A. B. Walker and A. Walsh. APL Materials 1, (2013) 9-14.

[201] I. Borriello, G. Cantele and D. Ninno. Physical Review B - Condensed Matter and Materials Physics, 77 (2008) 235214.

[202] K. Naoki, C. Yasuo, H. Liyuan. Jpn. J. Appl. Phys. 44 (2005) 4176.

[203] K. Wojciechowski, S.D. Stranks, A. Abate, G. Sadoughi, A. Sadhanala, N Kopidakis, G. Rumbles, C.Z. Li, R.H. Friend, A.K.Y. Jen, A.K.Y., H.J. Snaith. ACS Nano, 8 (2014) 12701-12709. 
[204] H. Nagaoka, F. Ma, D.W. de Quilettes, S.M. Vorpahl, M.S. Glaz, A.E. Colbert, M.E. Ziffer, D.S. Ginger, D.S. J. Phys. Chem. Lett. 6 (2015) 669-675.

[205] Z. Yuan, Z. Wu, S. Bai, Z. Xia, W. Xu, T. Song, H. Wu, L. Xu, J. Si, Y. Jin, B. Sun. Adv. Energy Mater (2015).

[206] H.J. Snaith, A. Abate, J.M. Ball, G.E. Eperon, T. Leijtens, N.K. Noel, N.K., S.D. Stranks, J.T.-W. Wang, K. Wojciechowski, W. Zhang. J. Phys. Chem. Lett. 5 (2014) 1511-1515.

[207] Unger, E.L., Hoke, E.T., Bailie, C.D., Nguyen, W.H., Bowring, A.R., Heumuller, T., Christoforo, M.G., McGehee, M.D. Energy Environ. Sci. 7 (2014) 3690-3698.

[208] Chen, H.-W., Sakai, N., Ikegami, M., Miyasaka, T. J. Phys. Chem. Lett. 6 (2014) 164-169.

[209] Wei, J., Zhao, Y., Li, H., Li, G., Pan, J., Xu, D., Zhao, Q., Yu, D. J. Phys. Chem. Lett. 5 (2014) 3937-3945.

[210] J.M. Frost, K.T. Butler, A. Walsh. APL Mater. 2 (2014).

[211] H. Zhang, C. Liang, Y. Zhao, M. Sun, H. Liu, J. Liang, D, Li, F. Zhang, Z. He. Phys. Chem. Chem. Phys. 17 (2015) 9613-9618.

[212] M. Zhang, H. Yu, M. Lyu, Q. Wang, J.H. Yun, L. Wang. J. Phys. Chem. C, 120 (2016) 2509-2519.

[213] J. You, Z. Hong, Y. Yang, Q. Chen, M. Cai, T.-B. Song, et al. ACS Nano, 8 (2014) 1674-1680.

[214] E. Edri, S. Kirmayer, M. Kulbak, G. Hodes, D. Cahen. J. Phys. Chem. Lett., 5 (2014) 429-433.

[215] S.A. Bretschneider, J. Weickert, J.A. Dorman, L. Schmidt-Mende. APL Mater., 2 (2014) 040701. 
[216] F. Deschler, M. Price, S. Pathak, L.E. Klintberg, D.-D. Jarausch, R. Higler, S. Hüttner, T. Leijtens, S.D. Stranks, H.J. Snaith, M. Atatüre, R.T. Phillips, R.H. Friend. J. Phys. Chem. Lett., 5 (2014) 1421-1426.

[217] G. Xing, N. Mathews, S.S. Lim, N. Yantara, X. Liu, D. Sabba, Dharani, M. Grätzel, S. Mhaisalkar, T.C. Sum. Nature Mater. 13 (2014) 1476.

[218] A. Sadhanala, F. Deschler, T.H. Thomas, S.E. Dutton, K.C. Goedel, F.C. Hanusch, M.L. Lai, U. Steiner, T. Bein, P. Docampo, D. Cahen, R.H. Friend. Phys. Chem. Lett., 5 (2014) 2501-2505.

[219] S.G. Carrero, R. E. Galian, J. P. Prieto. J. Mater. Chem. A, 3 (2015) 9187.

[220] K.L. Narasimhan, D. Kabra. ACS Appl. Mater. Interf. (2015). DOI: 10.1021/acsami.5b02159.

[221] L. Vegard. Z. Physik 5 (1921) 17.

[222] R.J. Elliott. Phys. Rev., 108 (1957) 1384.

[223] A. Dualeh, P. Gao, S.I. Seok, M.K. Nazeeruddin, M. Gratzel. Chem. Mater., 26 (2014) 6160-6164. https://doi.org/10.1021/cm502468k.

[224] Z. Cheng, J. Lin. Cryst. Eng. Comm., 12 (2010) 2646-2662. https://doi.org/10.1039/c001929a.

[225] G. Niu, W. Li, F. Meng, L. Wang, H. Dong, Y. Qiu. J. Mater. Chem. A, 2 (2013) 705-710. https://doi.org/10.1039/c3ta13606j.

[226] H.-L. Hsu, C.-C. Chang, C.-P. Chen, B.-H. Jiang, R.-J. Jeng, C.-H. Cheng. J. Mater. Chem. A, 3 (2015) 9271-9277. https://doi.org/10.1039/c5ta01563d.

[227] S.A. Kulkarni, T. Baikie, P.P. Boix, N. Yantara, N. Mathews, S. Mhaisalkar. J. Mater. Chem. A, 2 (2014) 9221-9225.

[228] G. Pellegrino, S. Colella, I. Deretzis, G.G. Condorelli, E. Smecca, G. Gigli, A. La Magna, A. Alberti. J. Phys. Chem. C, 119 (2015) 19808-19816. 
[229] J.R. Carvajal. Diffraction (IUCr), Newsletter, 26 (2001) 12-19.

[230] Y. Tian, I.G. Scheblykin. J. Phys. Chem. Lett., 6 (2015) 3466-3470.

[231] A.A. El-Shazly, M.M.H. El-Naby, M.A. Kenawy, M.M. El-Nahass, H.T. El-Shair, A.M. Ebrahim. Appl. Phys. A, 36 (1985) 51-53.

[232] R.J. Hill. Phys. C Solid State Phys., 7 (1974) 521-526.

[233] P. Fedeli, F. Gazza, D. Calestani, P. Ferro, T. Besagni, A. Zappettini, G. Calestani, E. Marchi, P. Ceroni, R. Mosca. J. Phys. Chem. C, 119 (2015) 21304-21313.

[234] J. He, T. Chen. J. Mater. Chem. A, 3 (2015) 18514-18520.

[235] L. Atourki, E. Vega, B. Marí, M. Mollar, H. Ait Ahsaine, K. Bouabid, A. Ihlal. Appl. Surf. Sci., 371 (2016) 112-117.

[236] X. Yang, X. Yan, W. Wang, X. Zhu, H. Li, W. Ma, C.X. Sheng. Org. Electron., 30 (2016) 79-83.

[237] M.I. Dar, N. Arora, P. Gao, S. Ahmad, M. Grätzel, M.K. Nazeeruddin. Nano Lett. 14 (2014) 6991-3996.

[238] N. Pellet, J. Teuscher, J.Maier, M. Grätzel. Chemistry of Materials, 27 (2015) $2181-2188$.

[239] M. Grätzel. Nature Materials, 13 (2014) 838-842.

[240] Y. Tidhar, E. Edri, H. Weissman, D. Zohar, G. Hodes, D. Cahen, B. Rybtchinski, S. Kirmayer. J. Am. Chem. Soc., 136 (2014) 13249-13255.

[241] K. Wang, C. Liu, P. Du, L. Chen, J. Zhu, A. Karim, X. Gong. Org. Electron. 21 (2015) 19-26.

[242] R.K. Misra, S. Aharon, B. Li, D. Mogilyansky, I.V.-Fisher, L. Etgar, E.A. Katz. J. Phys. Chem. Lett., 6 (2015) 326-330.

[243] Y. Chen, M. He, J. Peng, Y. Sun, Z. Liang. Adv. Sci., 3 (2016) 1500392. 
[244] E.L. Unger, A.R. Bowring, C.J. Tassone, V.L. Pool, A. Gold-Parker, R. Cheacharoen, K.H. Stone, E.T. Hoke, M.F. Toney, M.D. McGehee. Chem. Mater., 26 (2014) 7158-7165.

[245] J. Yan, B. Zhang, Y. Chen, A. Zhang, X. Ke. ACS Appl. Mater. Interfaces, 20 (2016) 12756-12763.

[246] H. Yu, F. Wang, F. Xie, W. Li, J. Chen, N. Zhao. Adv. Funct. Mater., 24 (2014) 7102-7108.

[247] Q. Chen, H. Zhou, Y. Fang, A.Z. Stieg, T.-B. Song, H.-H. Wang, X. Xu, Y. Liu, S. Lu, J. You, P. Sun, J. McKay, M.S. Goorsky, Y. Yang. Nat. Commun., 6 (2014) 7269 .

[248] S.T. Williams, F. Zuo, C.-C. Chueh, C.-Y. Liao, P.-W. Liang, A. K.-YJen. ACS Nano, 08 (2014) 10640-10654.

[249] E. Mosconi, A. Amat, M.K. Nazeeruddin, M. Grätzel, F. De Angelis. J. Phys. Chem. C, 117 (2013) 13902-13910.

[250] E. Vega, M. Mollar, B. Marí. Phys. Status Solidi C, 13 (2016) 30-34.

[251] G. Xing. Nat. Mater. 13 (2014) 476-480.

[252] W. Zhang, M. Saliba, D.T. Moore, S.K. Pathak, M.T. Hörantner, T. Stergiopoulos, S.D. Stranks, G.E. Eperon, J.A. Alexander-Webber, A. Abate, A. Sadhanala, S. Yao, Y. Chen, R.H. Friend, L.A. Estroff, U. Wiesner, H.J. Snaith. Nat. Commun., 6 (2015) 6142.

[253] M. Wei, Y.-H. Chung, Y. Xiao, Z. Chen. Org. Electron., 26 (2015) 206-264.

[254] L. Atourki, E. Vega, M. Mollar, B. Marí, H. Kirou, K. Bouabid, A. Ihlal. Journal of Alloys and Compounds 702 (2017) 404-409

[255] Q. Hao, Y. Chu, X. Zheng, Z. Liu, L. Liang, J. Qi, X. Zhang, G. Liu, H. Liu, H. Chen, C. Liu. J. Alloys Compd., 671 (2016) 11-16. 
[256] L. Atourki, E. Vega, B. Marí, M. Mollar, H.A. Ahsaine, K. Bouabid, A. Ihlal. Appl. Surf. Sci., 390 (2016) 744-750.

[257] G.E. Eperon, D. Bryant, J. Troughton, S.D. Stranks, M.B. Johnston, T. Watson, D.A. Worsley, H. Snaith. J. Phys. Chem. Lett., 6 (2015) 129.

[258] J.M. Frost, K.T. Butler, F. Brivio, C.H. Hendon, M. van Schilfgaarde. Nano Lett., 14 (2014) 2584-2590.

[259] J. Yang, B.D. Siempelkamp, D. Liu, T.L. Kelly. ACS Nano., 9 (2015) 1955-1963.

[260] N. Aristidou, I. Sanchez-Molina, T. Chotchuangchutchaval, M. Brown, L. Martinez, T. Rath, S.A. Haque. Angew. Chem. Int. Ed., 54 (2015) 8208-8212.

[261] C.C. Stoumpos, C.D. Malliakas, J.A. Peters, Z. Liu, M. Sebastian, J. Im, T.C. Chasapis, A.C. Wibowo, D.Y. Chung, A.J. Freeman, B.W. Wessels, M.G. Kanatzidis. Cryst. Growth. Des., 13 (2013) 2722-2727.

[262] M. Kulbak, D. Cahen, G. Hodes. J. Phys. Chem. Lett., 6 (2015) 2452-2456.

[263] R.J. Sutton, G.E. Eperon, L. Miranda, E.S. Parrott, B.A. Kamino, J.B. Patel, M.T. Horantner, M.B. Johnston. Adv. Energy Mater., 6 (2016) 1502458.

[264] M. Wang, G.T. Fei, Y.G. Zhang, M.G. Kong, L.D. Zhang. Adv. Mater., 19 (2007) 4491-4494.

[265] G.E. Eperon, G.M. Paterno, R.J. Sutton, A. Zampetti, A.A. Haghighirad, F. Cacialli, H.J. Snaith. J. Mater. Chem. A, 3 (2015) 19688-19695.

[266] M. Sauer, J. Hofkens, J. Enderlein. John Wiley \& Sons, (2010).

[267] Y. Rakita, N. Kedem, S. Gupta, A. Sadhanala, V. Kalchenko, M.L. Bohm, M. Kulbak, R.H. Friend, D. Cahen, G. Hodes. Cryst. Growth. Des., 16 (2016) $5717-5725$

[268] B. Conings, J. Drijkoningen, N. Gauquelin, A. Babayigit, J. D'Haen, L. D’Olieslaeger, A. Ethirajan, J. Verbeeck, J. Manca, E. Mosconi, F. De Angelis, H.-G. Boyen. Adv. Energy Mater., 5 (2015) 1500477. 
[269] M. Shirayama, M. Kato, T. Miyadera, T. Sugita, T. Fujiseki, S. Hara, H. Kadowaki, D. Murata, M. Chikamatsu, H. Fujiwara. J. Appl. Phys., 119 (2016) 115501.

[270] M. Kulbak, S. Gupta, N. Kedem, I. Levine, T. Bendikov, Gary Hodes, D. Cahen. J. Phys. Chem. Lett., 7 (2016) 167-172.

[271] E. Vega, M. Mollar, B. Mari. Journal of Alloys and Compounds 739 (2018) 1059-1064.

[272] B.V. Lotsch. Angew. Chem. Int. Ed., $53 \quad$ (2014) 635-637. https://doi.org/10.1002/anie.201309368.

[273] N.-G. Park. J. Phys. Chem. Lett., $4 \quad$ (2013) 2423-2429. https://doi.org/10.1021/jz400892a.

[274] R. Dhanker, A.N. Brigeman, A.V. Larsen, R.J. Stewart, J.B. Asbury, N.C. Giebink. Appl. Phys. Lett., $105 \quad$ (2014) 151112. https://doi.org/10.1063/1.4898703.

[275] H.J. Snaith, M. Gratzel. Adv. Mater., 19 (2007) 3643-3647.

[276] Y. Xiao, G. Han, Y. Chang, H. Zhou, M. Li, Y. Li. J. Power Sources, 267 (2014) $1-8$.

[277] S.N. Habisreutinger, T. Leijtens, G.E. Eperon, S.D. Stranks, R.J. Nicholas, H.J. Snaith. Nano Lett., 14 (2014) 5561. https:// doi.org/10.1021/nl501982b.

[278] I. Hwang, I. Jeong, J. Lee, M.J. Ko, K. Yong. ACS Appl. Mater. \& Interfaces, 7 (2015) 17330. https://doi.org/10.1021/acsami.5b04490.

[279] W.-J. Yin, T. Shi, Y. Yan. Appl. Phys. Lett., 104 (2014) 63903. https://doi.org/10.1063/1.4864778.

[280] J. Kim, S.-H. Lee, J.H. Lee, K.-H. Hong. J. Phys. Chem. Lett., 5 (2014) 1312-1317. https://doi.org/10.1021/jz500370k. 
[281] D.W. de Quilettes, S.M. Vorpahl, S.D. Stranks, H. Nagaoka, G.E. Eperon, M.E. Ziffer, H.J. Snaith, D.S. Ginger. Science, 348 (2015) 683-686.

[282] M.L. Agiorgousis, Y.-Y. Sun, H. Zeng, S. Zhang. J. Am. Chem. Soc., 136 (2014) 14570-14575, https://doi.org/10.1021/ja5079305.

[283] A. Abate, M. Saliba, D.J. Hollman, S.D. Stranks, K. Wojciechowski, R. Avolio, G. Grancini, A. Petrozza, H.J. Snaith. Nano Lett., 14 (6) (2014) 3247-3254.

[284] N.K. Noel, A. Abate, S.D. Stranks, E.S. Parrott, V.M. Burlakov, A. Goriely, H.J. Snaith. ACS Nano, 8(10) (2014) 9815-9821.

[285] W.-J. Yin, H. Chen, T. Shi, S.-H. Wei, Y. Yan. Adv. Electron. Mater., 1(6) (2015) 1500044. https://doi.org/10.1002/aelm.201500044.

[286] G.Y. Kim, S.H. Oh, B.P. Nguyen, W. Jo, B.J. Kim, D.G. Lee, H.S. Jung. J. Phys. Chem. Lett. 6 (2015) 2355-2362. https://doi.org/10.1021/acs.jpclett.5b00967.

[287] M. Szafraski, M. Jarek. Cryst. Eng. Comm., 15 (2013) 4617-4623. https://doi.org/10.1039/c3ce26979e.

[288] N. De Marco, H. Zhou, Q. Chen, P. Sun, Z. Liu, L. Meng, E.-P. Yao, Y. Liu, A. Schiffer, Y. Yang. Nano Lett., 16 (2016) 1009-1016. https://doi.org/10.1021/acs.nanolett.5b04060.

[289] G. Giorgi, K. Yamashita. Nanotechnology, $26 \quad(2015) \quad 442001$. https://doi.org/10.1088/0957-4484/26/44/442001.

[290] C. Aranda, C. Cristobal, L. Schooshtari, S. Huettner, A. Guerrero. Sustainable Energy and Fuels, 1 (2017) 540-547.

[291] G. Wang, D. Liu, J. Xiang, D. Zhou, K. Alameh, B. Dinga, Q. Song. RSC Adv., 6 (2016) 43299 


\section{List of Publications}

The realization of this thesis has led to the publication of the following articles, whose results, expanded and revised, constitute the core of this text.

- Erika Vega, Miguel Mollar, Bernab Mar. Effect of guanidinium on the optical properties and structure of the methylammonium lead halide perovskite. Journal of Alloys and Compounds 739 (2018) 1059-1064.

- Atourki, Lahoucine; Vega-Fleitas, Erika; Mar, B.; Mollar Garca, Miguel Alfonso. Impact of iodide substitution on the physical properties and stability of cesium lead halide perovskite thin films $\mathrm{CsPbBr}_{3-x} I_{x}(0 \leq$ $x \leq 1$ ). Journal of Alloys and Compounds (ISSN 0925-8388) 702, 404 409. (2017)

- Atourki, Lahoucine; Vega-Fleitas, Erika; Mar, B.; Mollar Garca, Miguel Alfonso; Ait Ahsaine, H; Bouabid, K; Ihlal, A. $M A P b I_{2.9-x} B r_{x} C l_{0.1}$ hybrid halide perovskites: Shedding light on the effect of chloride and bromide ions on structural and photoluminescence properties. Applied Surface Science (ISSN 0169-4332) 390, 744 - 750. (2016)

- L. Atourki; Vega-Fleitas, Erika; Mar Soucase, Bernab; Mollar Garca, Miguel Alfonso; A. Ihlal. Role of the chemical substitution on the structural and luminescence properties of the mixed halide perovskite thin $\mathrm{MAPbI}_{3-x} B r_{x} 0 \leq x \leq 1$ films. Applied Surface Science (ISSN 0169-4332) 371, 112 - 117. (2016)

- Vega-Fleitas, Erika; Mollar Garca, Miguel Alfonso; Mar Soucase, Bernab. Synthesis of $\mathrm{MAPbBr}_{3-i} Y_{i}(Y=I, C l ; i=0,1,2,3)$ perovskite thin films. Physica Status Solidi (C) (ISSN 1610-1634) 13, 30 - 34. (2016) 

